University of Cardiff

\title{
Urban Morphology and Housing Market
}

\author{
A thesis submitted in partial fulfillment for the degree of \\ Doctor of Philosophy (PhD)
}

By

Yang Xiao

B.Sc in Urban Planning

Southeast University (2007)

M.Arch in Urban Design

University College London (2008)

October 2012 


\section{APPENDIX 1:}

Specimen layout for Thesis Summary and Declaration/Statements page to be included in a Thesis

\section{DECLARATION}

This work has not previously been accepted in substance for any degree and is not concurrently submitted in candidature for any degree.

Signed (candidate)

Date

\section{STATEMENT 1}

This thesis is being submitted in partial fulfillment of the requirements for the degree of ......................(insert MCh, MD, MPhil, $\mathrm{PhD}$ etc, as appropriate)

Signed (candidate)

Date

\section{STATEMENT 2}

This thesis is the result of my own independent work/investigation, except where otherwise stated. Other sources are acknowledged by explicit references.

Signed (candidate)

Date

\section{STATEMENT 3}

I hereby give consent for my thesis, if accepted, to be available for photocopying and for inter-library loan, and for the title and summary to be made available to outside organisations.

Signed (candidate)

Date

\section{STATEMENT 4: PREVIOUSLY APPROVED BAR ON ACCESS}

I hereby give consent for my thesis, if accepted, to be available for photocopying and for inter-library loans after expiry of a bar on access previously approved by the Graduate Development Committee.

Signed (candidate)

Date 


\begin{abstract}
Urban morphology has been a longstanding field of interest for geographers but without adequate focus on its economic significance. From an economic perspective, urban morphology appears to be a fundamental determinant of house prices since morphology influences accessibility. This $\mathrm{PhD}$ thesis investigates the question of how the housing market values urban morphology. Specifically, it investigates people's revealed preferences for street patterns. The research looks at two distinct types of housing market, one in the UK and the other in China, exploring both static and dynamic relationships between urban morphology and house price. A network analysis method known as space syntax is employed to quantify urban morphology features by computing systemic spatial accessibility indices from a model of a city's street network. Three research questions are empirically tested. Firstly, does urban configuration influence property value, measured at either individual or aggregate (census output area) level, using the Cardiff housing market as a case study? The second empirical study investigates whether urban configurational features can be used to better delineate housing submarkets. Cardiff is again used as the case study. Thirdly, the research aims to find out how continuous change to the urban street network influences house price volatility at a micro-level. Data from Nanjing, China, is used to investigate this dynamic relationship. The results show that urban morphology does, in fact, have a statistically significant impact on housing price in these two distinctly different housing markets. I find that urban network morphology features can have both positive and negative impacts on housing price. By measuring different types of connectivity in a street network it is possible to identify which parts of the network are likely to have negative accessibility premiums (locations likely to be congested) and which parts are likely to have positive premiums (locations highly connected to destination opportunities). In the China case study, I find that this relationship holds dynamically as well as statically, showing evidence that price change is correlated with some aspects of network change.
\end{abstract}




\section{Dedication}

I would like to dedicate this thesis to my wife and son,

who give me unconditional love, sacrifice, encouragement and propulsion for learning. 


\section{Acknowledgements}

I have spent almost three years to complete this research, in fact, during these time I am not fighting the war of $\mathrm{PhD}$ independently. This work would not have been completed without the great support and sincere help from many people.

First of all, I would like to thank my supervisors Prof. Chris Webster and Dr. Scott Orford for developing my knowledge of urban economics, and commenting and correcting successive drafts of the thesis in every detail, as well as their support, invaluable advice, patient guidance, encouragement and thoughtfulness through the completion of this study,

I would also like to thank Prof. Fulong Wu, Prof. Eric Heikkila, Alain Chiaradia, Dr. Yiming Wang, Dr. Fangzhu Zhang, Prof. Zhigang Li, and Prof. Xiaodong Song, for their insightful comments and advice, as well as their encouragement. I am grateful to all of the faculty and staff in the Cardiff school of planning and geography for their help.

I owe many thanks to my doctoral colleagues, especially Chris Zheng Wang, Chinmoy Sarkar, Agata Krause, Amanda Scarfi, Kin Wing Chan, II Hyung Park, Tianyang Ge, Dr. Jie Shen and Dr. Yi Li, for their help on improving my research. Many thanks should be given to Chris Zheng Wang's family, Xiaoyu Zhang, Chunquan Yu, Fan Ye, and Xin Yu, for offering their priceless friendship, hospitality, and practical help. I am grateful to all of my friends who are not physically around me but encourage me all the time.

Finally, I would like to thank my mother, my parents, parents-in-law, particularly my wife Mrs. Lu Liu and my son Sean Yaru Liu, for their supplication, support, sacrifice and encouragement throughout my life. 


\section{Contents}

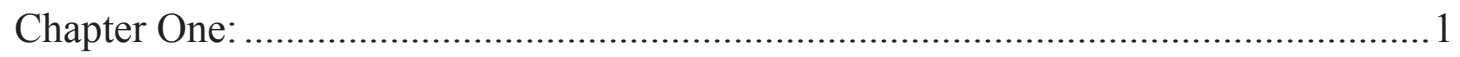

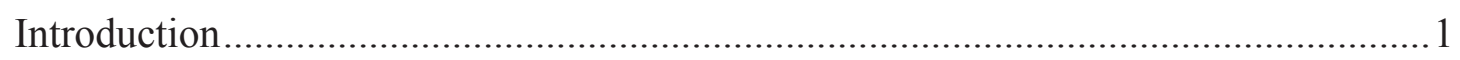

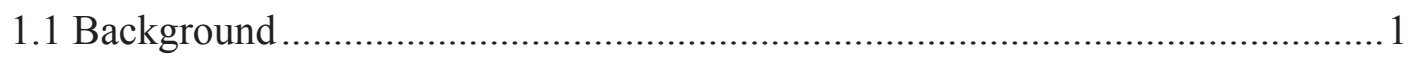

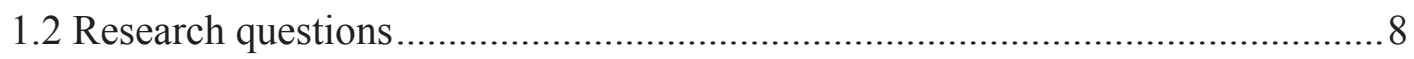

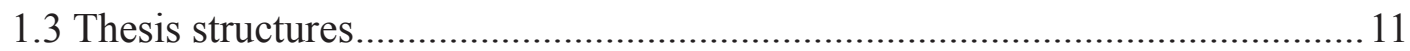

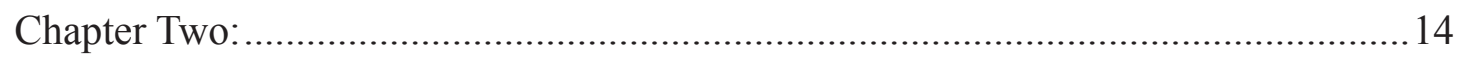

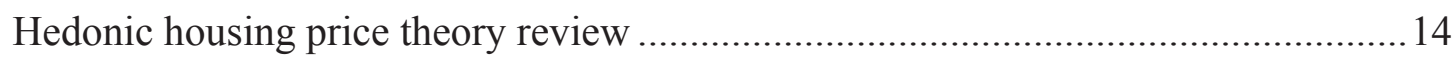

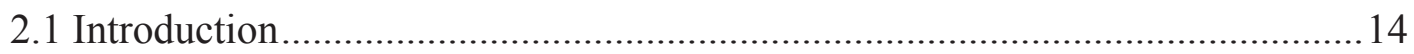

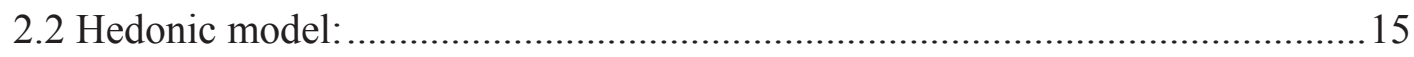

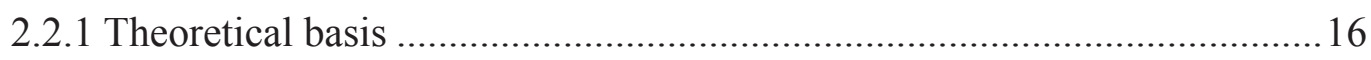

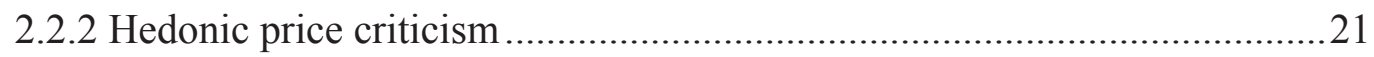

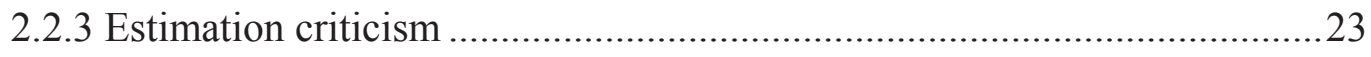

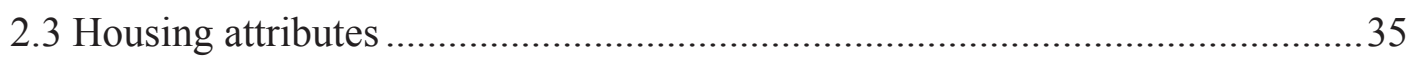

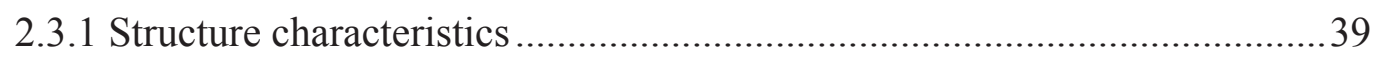

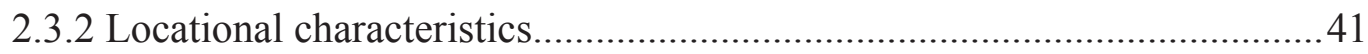

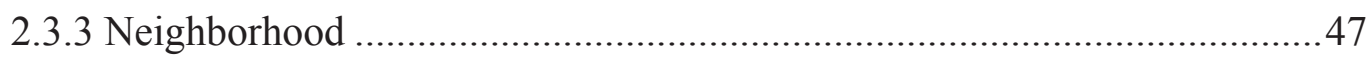

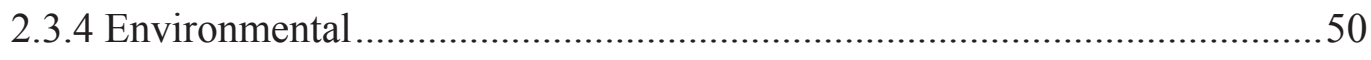

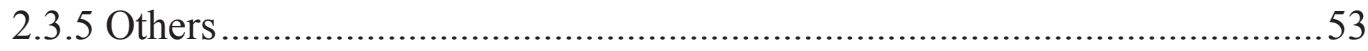

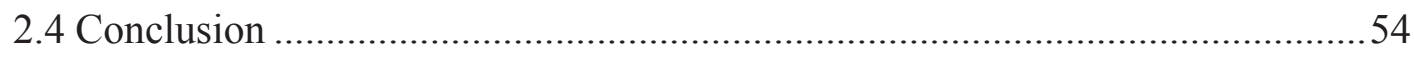

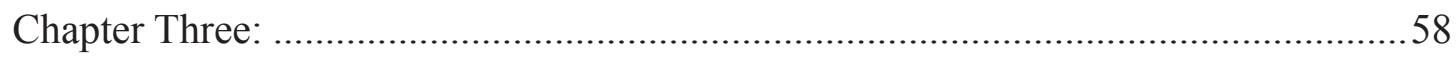




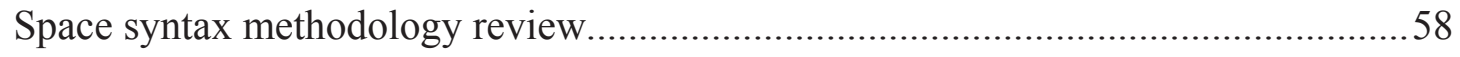

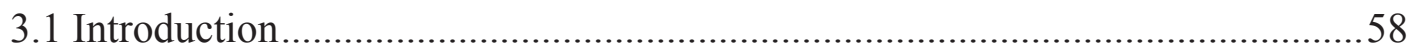

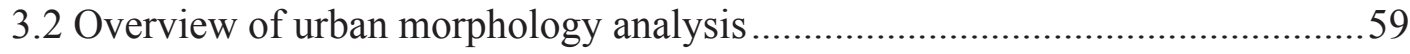

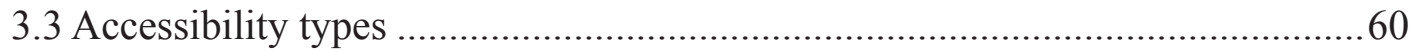

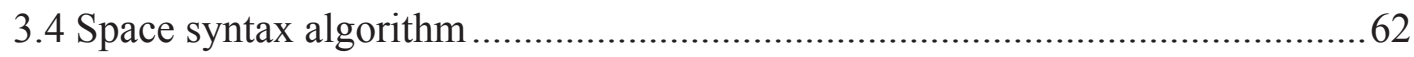

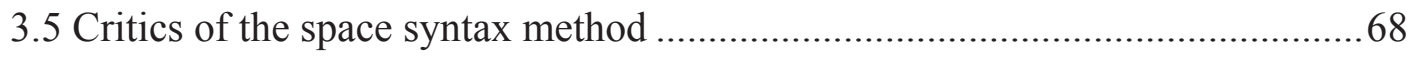

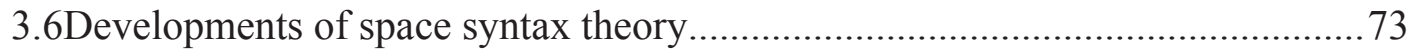

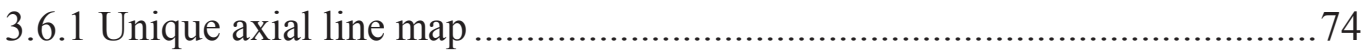

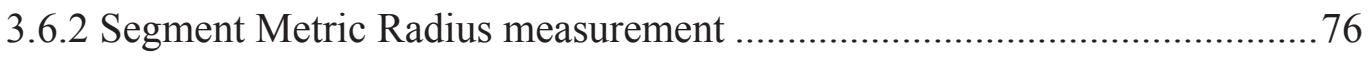

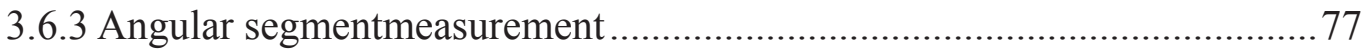

3.7 How urban morphology interacts with social economics phenomenon ............78

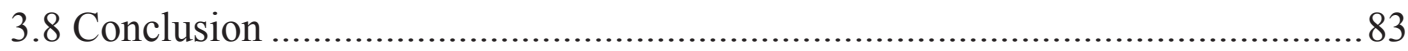

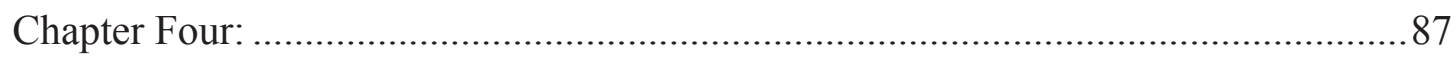

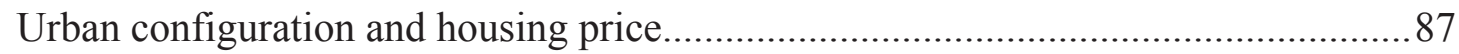

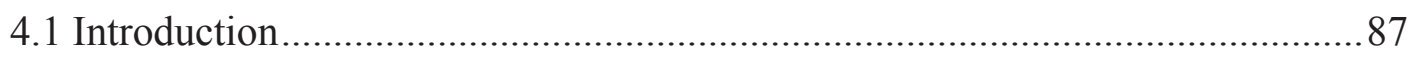

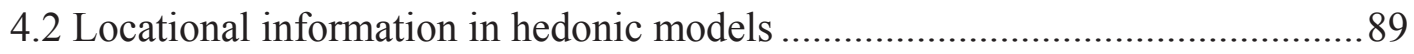

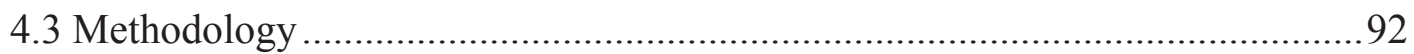

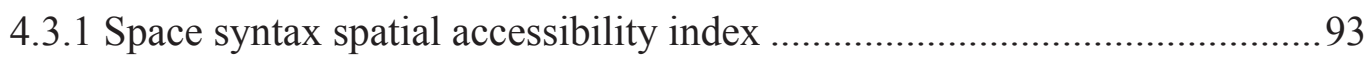

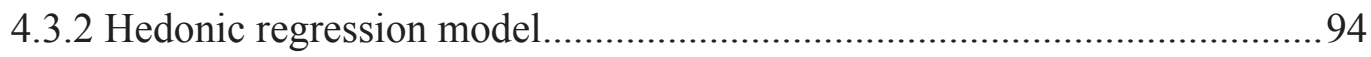

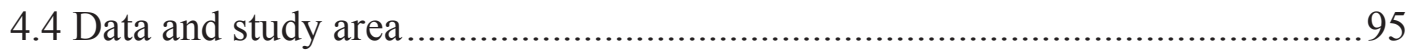

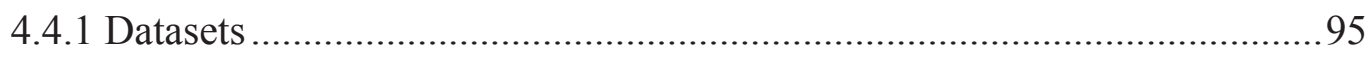

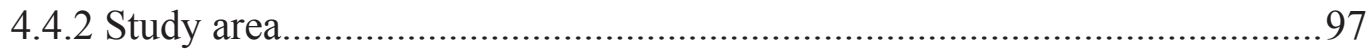

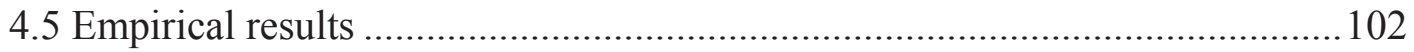

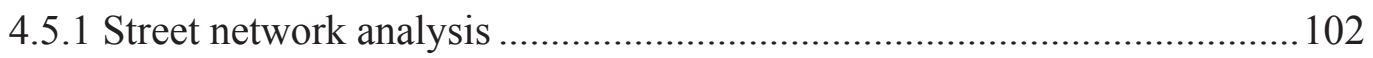


4.5.2 Disaggregated data.....

4.5.3 Aggregate data

4.5.4 Discussion of disaggregated data and aggregated data....

4.6 Conclusion

Chapter Five:

Identification of housing submarkets by urban configurational features

5.1 Introduction

5.2 Literature review

5.2.1Specifications of housing submarket

5.2.2 Accessibility and social neighborhood characteristics

5.3 Methodologies

5.3.1 Space syntax

5.3.2 Hedonic price model

5.3.3 Two-Step cluster analysis

5.3.4 Chow test

5.3.5 Weighted standard error estimation

5.4 Study area and dataset.

5.5 Empirical analysis

5.5.1 Market-wide hedonic model

5.5.2 Specifications and estimations for submarkets

5.5.3 Estimation of weighed standard error 160

5.6 Conclusions 
Identifying the micro-dynamic effects of urban street configuration on house price

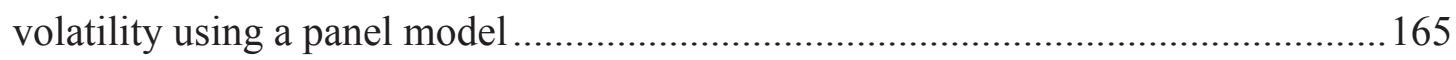

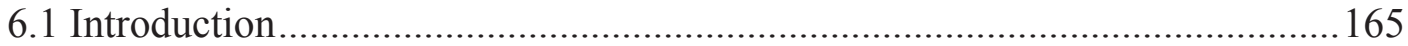

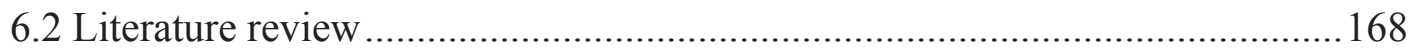

6.2.1 Cross-sectional static house price models............................................. 168

6.2.2 Hybrid repeat sales model with hedonic model ......................................169

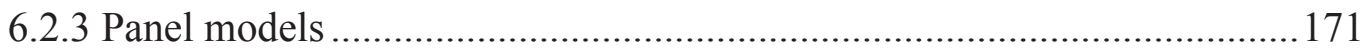

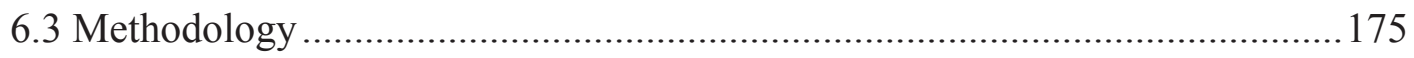

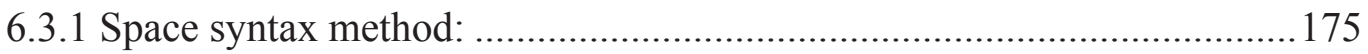

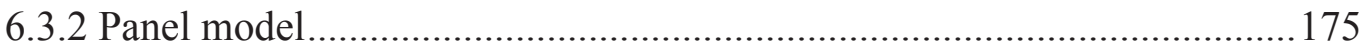

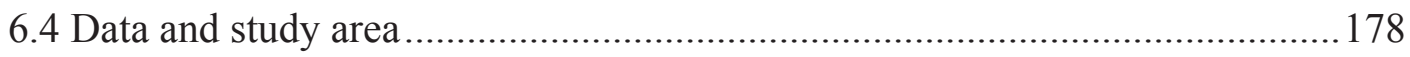

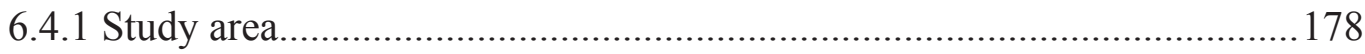

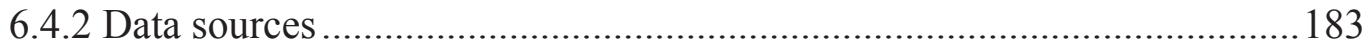

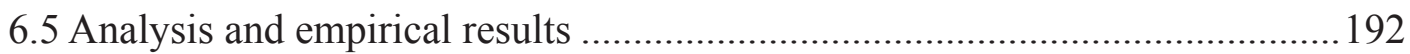

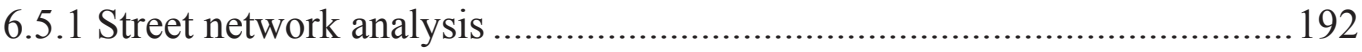

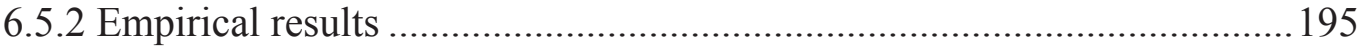

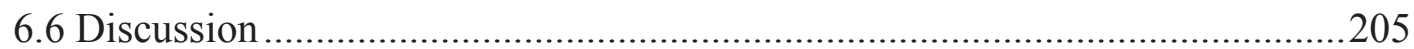

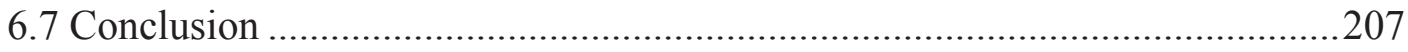

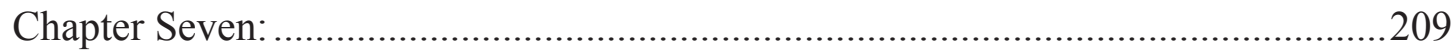

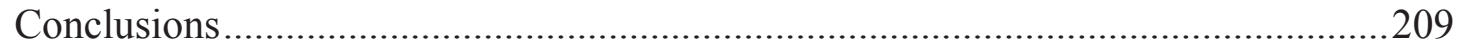

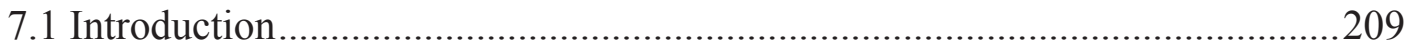

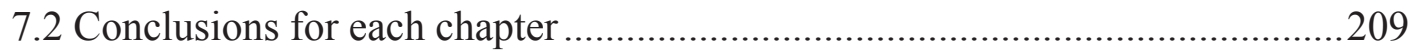

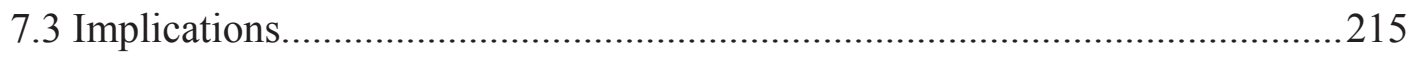

7.3.1 Implications for the Space Syntax theory ............................................215 


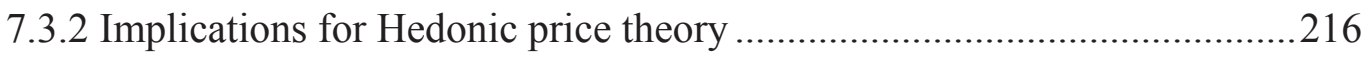

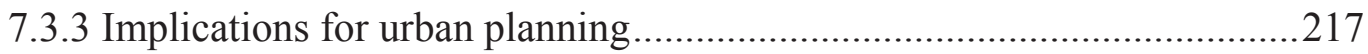

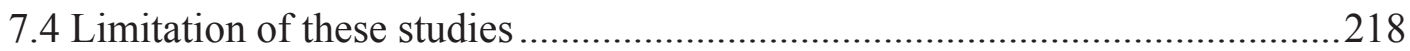

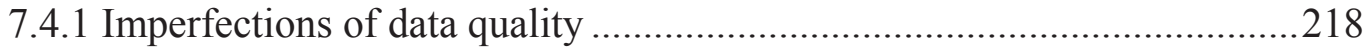

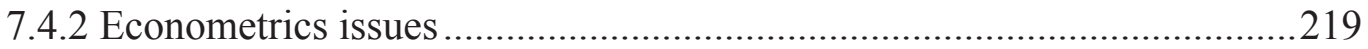

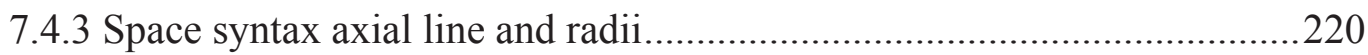

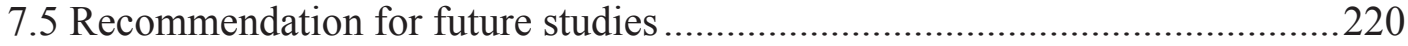

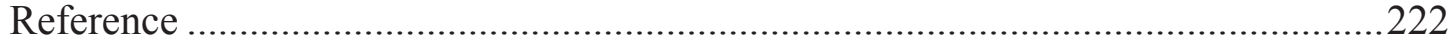

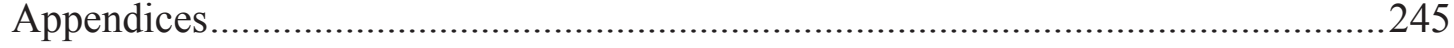




\section{List of Tables}

Table 2. 1 Selected previous studies on hedonic price model..............................37

Table 4. 1 The transaction number of each year ............................................... 99

Table 4. 2 Fifty-five variables and Description ................................................ 101

Table 4. 3 Descriptive Statistics for disaggregated dataset.............................. 103

Table 4. 4 Regression results of Model I (a) and (b) ........................................ 107

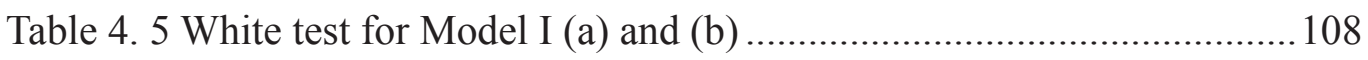

Table 4. 6 Global Moran's I for Model I (a) and (b) ........................................ 108

Table 4. 7 Model I (c): Different Radii - T value comparisons.......................... 110

Table 4. 8 Descriptive Statistics for aggregated dataset................................... 111

Table 4. 9 Regression results of Model II (a) and (b) ..................................... 115

Table 4. 10 White test for Model II (a) and (b) ............................................... 116

Table 4. 11 Global Moran's I for Model II (a) and (b)..................................... 116

Table 4. 12 Model II (c): Different Radii - T value comparisons ...................... 118

Table 4. 13 Comparison the results with previous studies ...............................120

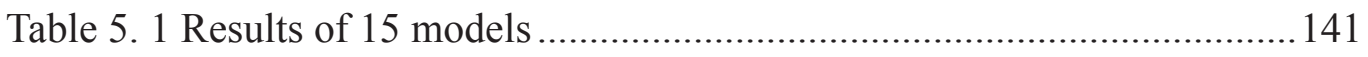

Table 5. 2 Estimation results of dwelling type specification ........................... 143

Table 5. 3 Chow test results of dwelling type specification ............................ 144

Table 5. 4 Estimation results of spatial nested specification ........................... 147

Table 5. 5 Chow test results of spatial nested specification............................... 148

Table 5. 6 Cluster results of optimal urban configurational features specification 149

Table 5. 7 Descriptive of four submarkets 152

Table 5. 8 Estimation results of optimal urban configuration specification ......154

Table 5. 9 Chow test results of optimal urban configuration specification........154

Table 5. 10 Cluster results of nested urban configuration and building type

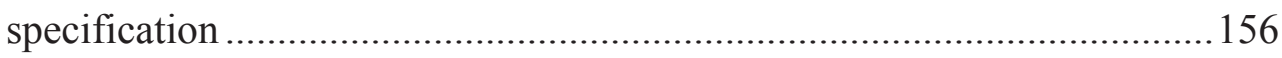

Table 5. 11 Descriptive of five submarkets.................................................. 158

Table 5. 12 Estimation results of nested all urban configurational features and 
building type specification

Table 5. 13 Chow test results of nested all urban configurational features and building type specification...... 160

Table 5. 14 Estimation results of weighed standard error................................ 161

Table 6. 1 General information of Nanjing from 2005-2010 ......................... 182

Table 6. 2 The changes of accessibility at different radii from 2005 to $2010 \ldots .186$

Table 6.3 The changes of mean of housing price from 2005 to 2010 ...............187

Table 6. 4 Statistics descriptive data ............................................................. 189

Table 6. 5 Empirical results of five models ......................................................195

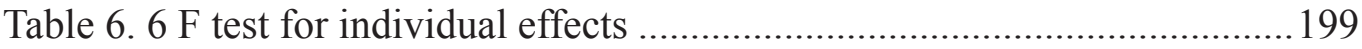

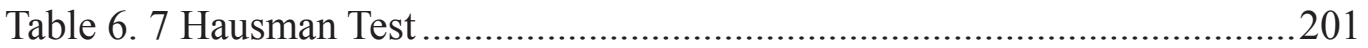

Table 6. $8 \mathrm{~F}$ test for individual effects and time-fixed effect ...........................203

Table 6. 9 Lagrange Multiplier Test...............................................................204 


\section{List of Figures}

Figure 1. 1 Rent price pattern in Seattle ........................................................... 5

Figure 2. 1 Demand and offer curves of hedonic price function ....................... 18

Figure 2. 2 The marginal implicit price of an attribute as a function of supply and

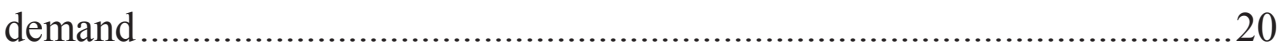

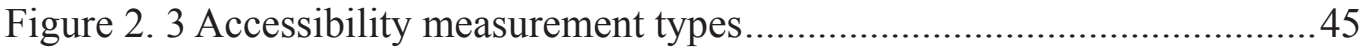

Figure 3. 1 Conventional graph-theoretic representation of the street network ..62

Figure 3. 2 The process of converting the "Convex Space " to axial line map....64

Figure 3.3 calculation of depth value of each street ........................................65

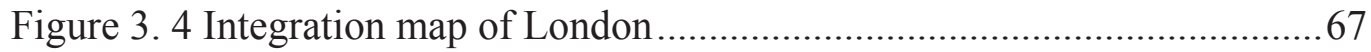

Figure 3. 5 Value changes when deform the configuration ............................... 71

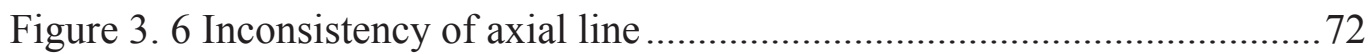

Figure 3. 7 Cross error for two axial line maps ............................................... 73

Figure 3. 8 An algorithmic definition of the axial map..................................... 74

Figure 3. 9 Definition of axial line by AxialGen ...............................................75

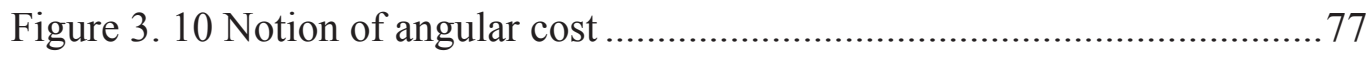

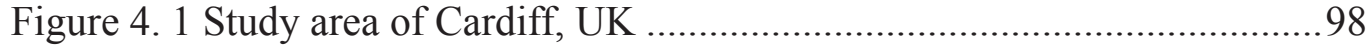

Figure 4. 2 The Std. Dev. of housing price in output area units ....................... 100

Figure 4. 4 Locational characteristics t value change for model I (c)............... 111

Figure 4. 5 Locational characteristics t value change for model II (c) .............. 118

Figure 5. 1 The $\mathrm{t}$ value change of all the variables via 15 models ....................142

Figure 5. 2 Two-step cluster result of urban configuration feactures at $7 \mathrm{~km}$.... 149

Figure 5. 3 Two-step cluster result of nested dwelling type and all urban

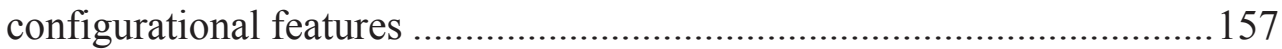

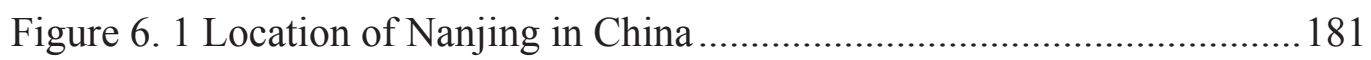

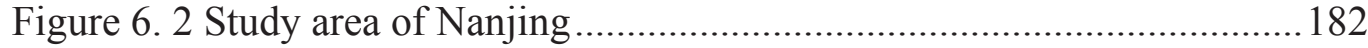

Figure 6. 3 The changes of urban configuration in Nanjing from 2005-2010 ... 184 
Figure 6. 4 Integration value change from 2005-2010 at different radii. 193

Figure 6. 5 Choice value change from 2005-2010 at different radii. 194

Figure 7. 1 Diagrammatic structural equation of housing price 214

Figure A4. 1Integration at radii of $0.4 \mathrm{~km}$ 246

Figure A4. 2 Integration at radii of $0.8 \mathrm{~km}$ 246

Figure A4. 3 Integration at radii of $1.2 \mathrm{~km}$ 246

Figure A4. 4 Integration at radii of $1.6 \mathrm{~km}$ .246

Figure A4. 5 Choice at radii of $0.4 \mathrm{~km}$ 246

Figure A4. 6 Choice at radii of $0.8 \mathrm{~km}$ 246

Figure A4. 7 Choice at radii of $1.2 \mathrm{~km}$ 246

Figure A4. 8 Choice at radii of $1.6 \mathrm{~km}$ 246

Figure A4. 9 Integration at radii of $2 \mathrm{~km}$ 247

Figure A4. 10 Integration at radii of $2.5 \mathrm{~km}$ 247

Figure A4. 11 Integration at radii of $3 \mathrm{~km}$ 247

Figure A4. 12 Integration at radii of $4 \mathrm{~km}$ 247

Figure A4. 13 Choice at radii of $2 \mathrm{~km}$ .247

Figure A4. 14 Choice at radii of $2.5 \mathrm{~km}$ 247

Figure A4. 15 Choice at radii of $3 \mathrm{~km}$ 247

Figure A4. 16 Choice at radii of $4 \mathrm{~km}$ 247

Figure A4. 17 Integration at radii of $5 \mathrm{~km}$ 248

Figure A4. 18 Integration at radii of $6 \mathrm{~km}$ 248

Figure A4. 19 Integration at radii of $7 \mathrm{~km}$ 248

Figure A4. 20 Integration at radii of $8 \mathrm{~km}$ .248

Figure A4. 21 Choice at radii of $5 \mathrm{~km}$ 248

Figure A4. 22 Choice at radii of $6 \mathrm{~km}$ .248

Figure A4. 23 Choice at radii of $7 \mathrm{~km}$ 248

Figure A4. 24 Choice at radii of $8 \mathrm{~km}$ .248

Figure A4. 25 Integration at radii of $10 \mathrm{~km}$ 249

Figure A4. 26 Global integration 249 


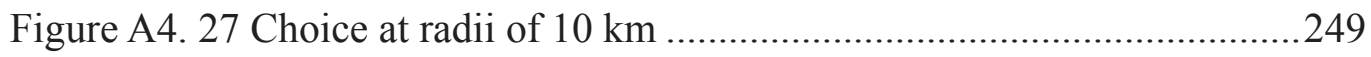

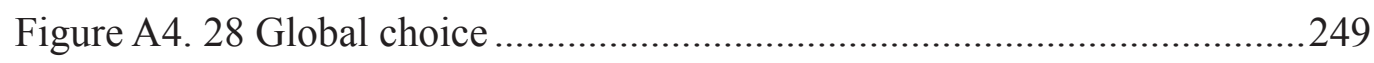

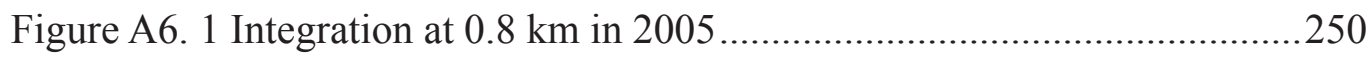

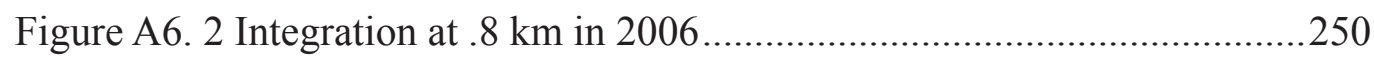

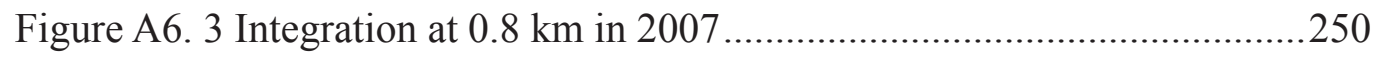

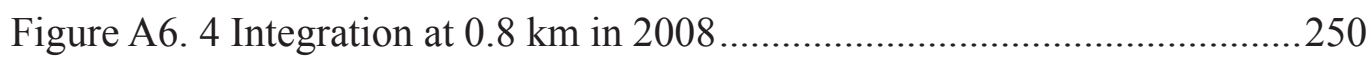

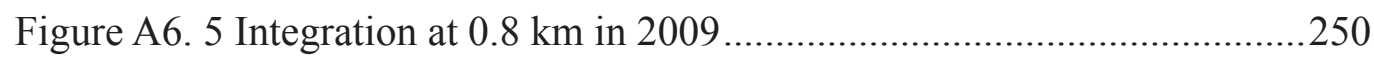

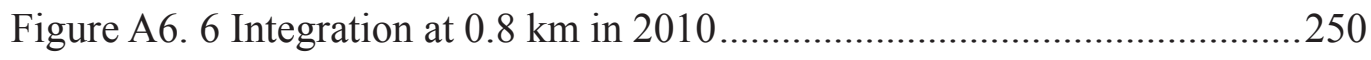

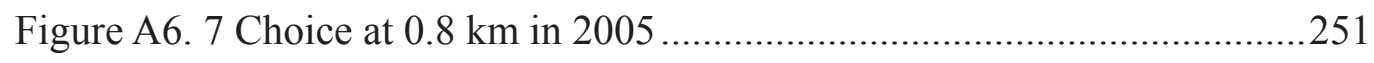

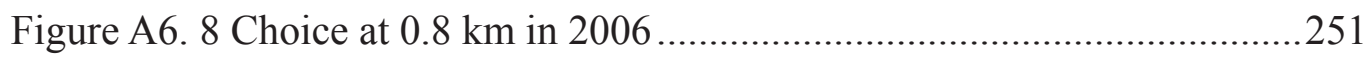

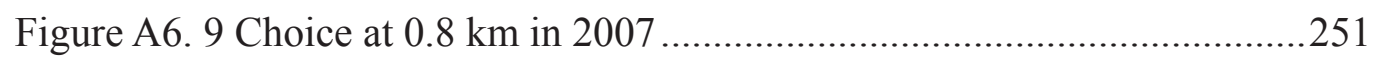

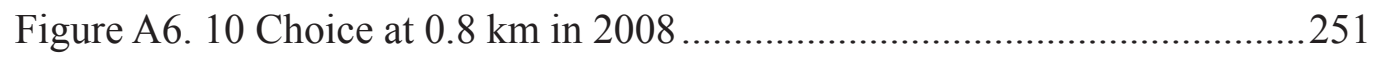

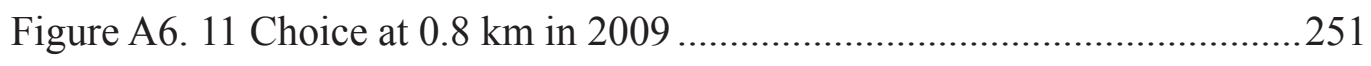

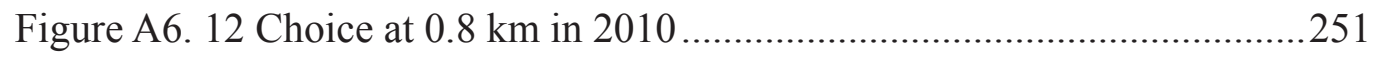

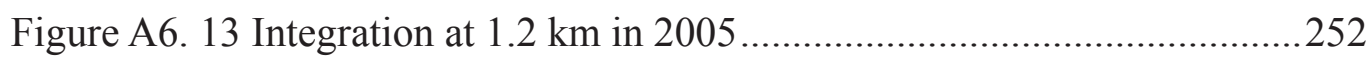

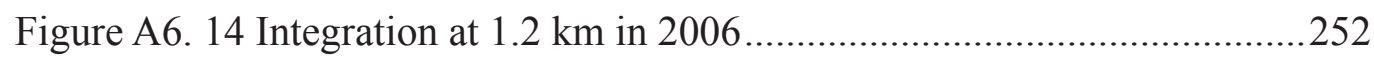

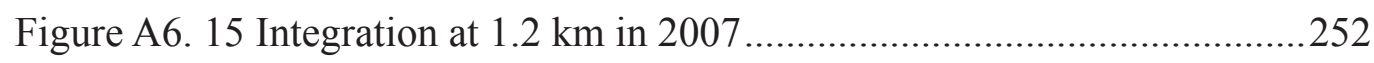

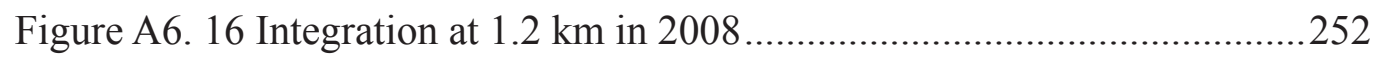

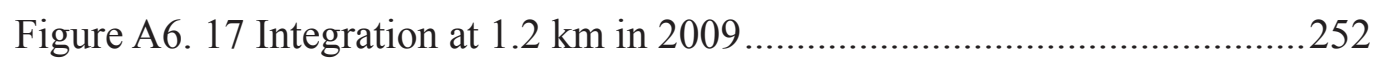

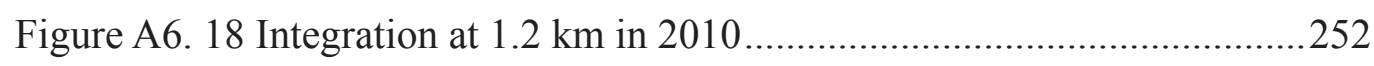

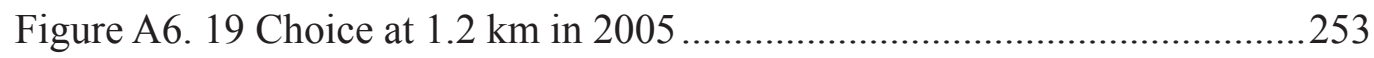

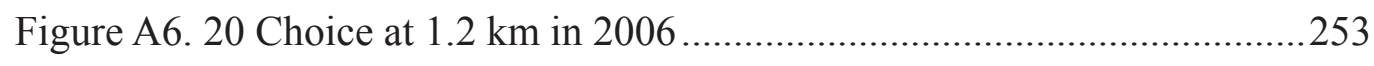

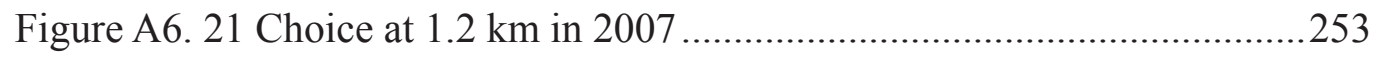

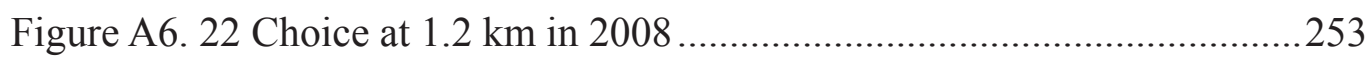

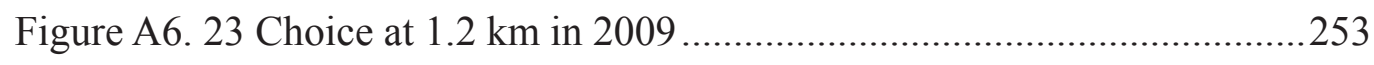

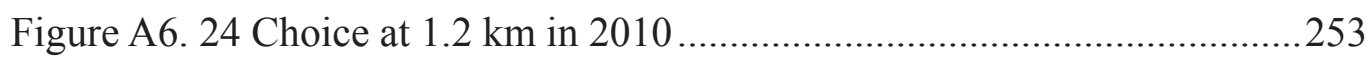

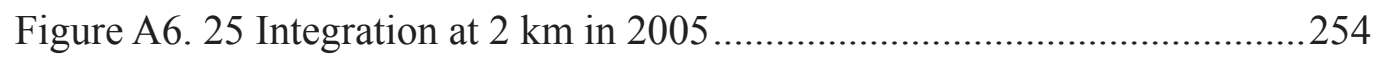

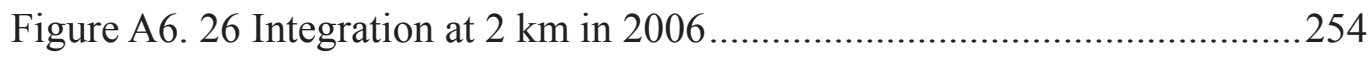

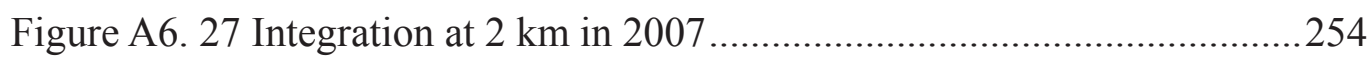




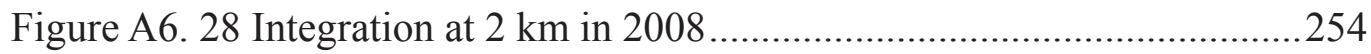

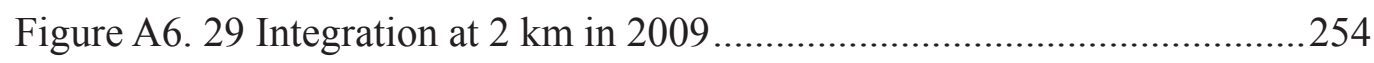

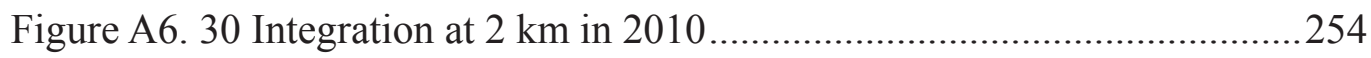

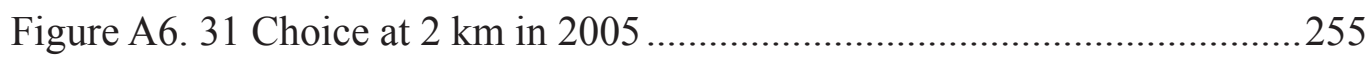

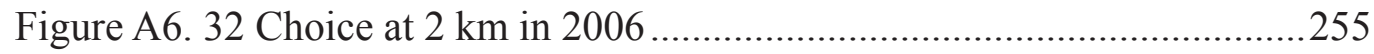

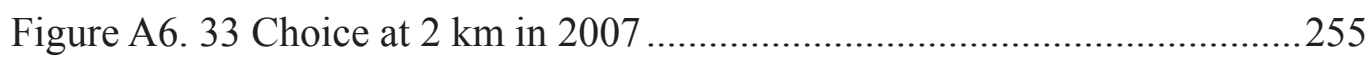

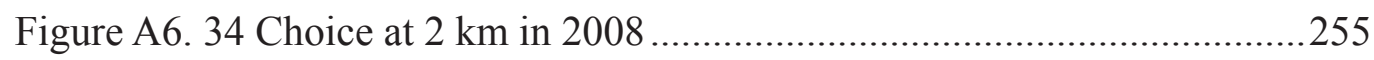

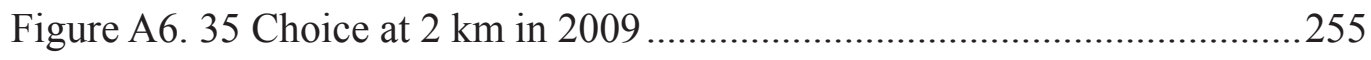

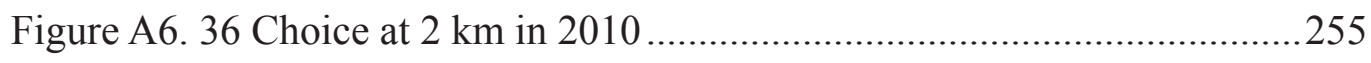

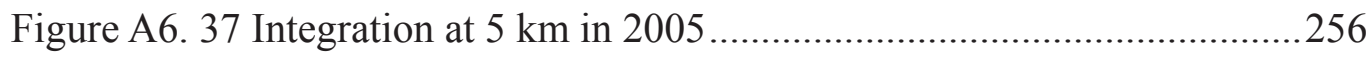

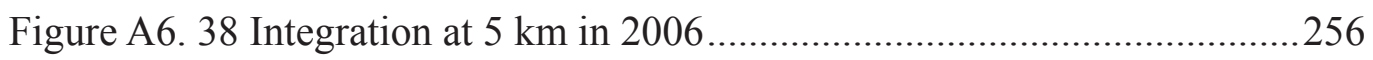

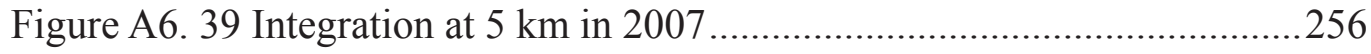

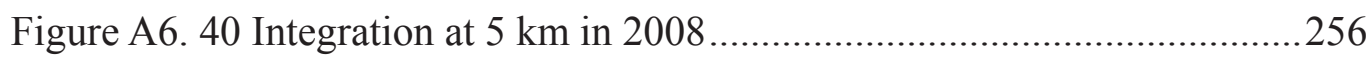

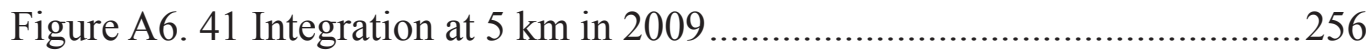

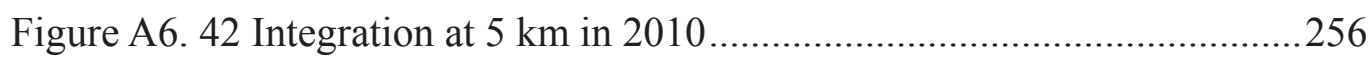

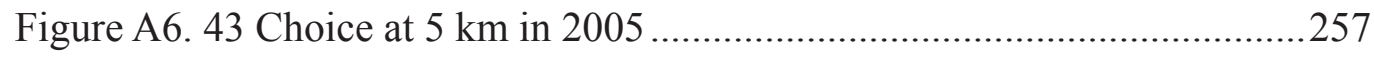

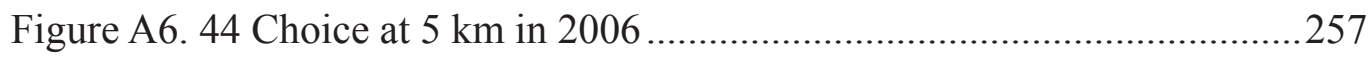

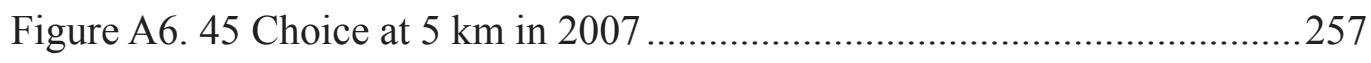

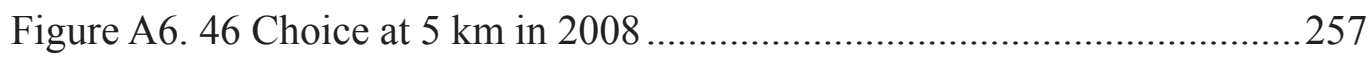

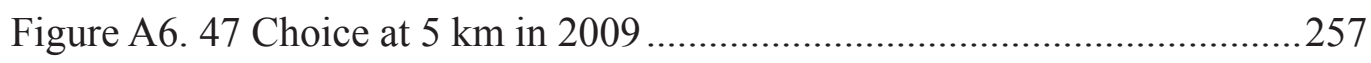

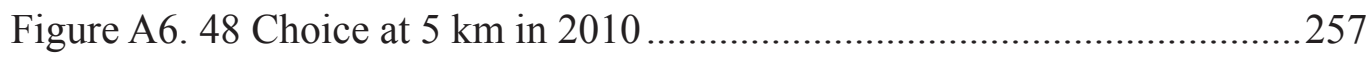

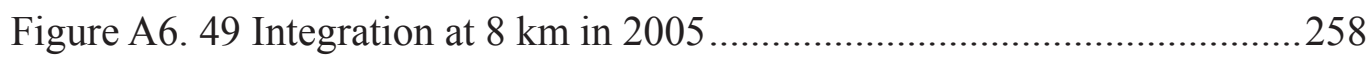

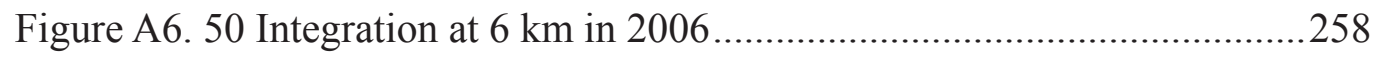

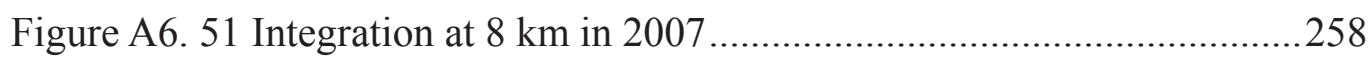

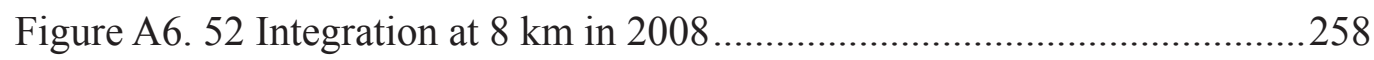

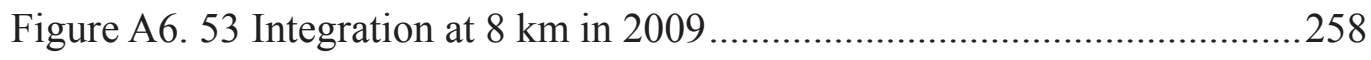

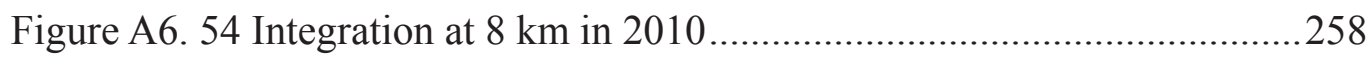

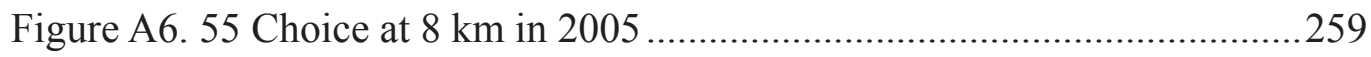

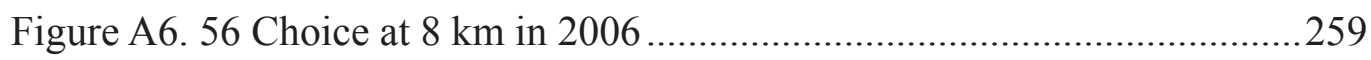


Figure A6. 57 Choice at $8 \mathrm{~km}$ in 2007

Figure A6. 58 Choice at $8 \mathrm{~km}$ in 2008 259

Figure A6. 59 Choice at $8 \mathrm{~km}$ in 2009 259

Figure A6. 60 Choice at $8 \mathrm{~km}$ in 2010 259

Figure A6. 61 Integration at $10 \mathrm{~km}$ in 2005 260

Figure A6. 62 Integration at $10 \mathrm{~km}$ in 2006 260

Figure A6. 63 Integration at $10 \mathrm{~km}$ in 2007 .260

Figure A6. 64 Integration at $10 \mathrm{~km}$ in 2008 260

Figure A6. 65 Integration at $10 \mathrm{~km}$ in 2009 .260

Figure A6. 66 Integration at $10 \mathrm{~km}$ in 2010. 260

Figure A6. 67 Choice at $10 \mathrm{~km}$ in 2005 261

Figure A6. 68 Choice at $10 \mathrm{~km}$ in 2006 261

Figure A6. 69 Choice at $10 \mathrm{~km}$ in 2007 261

Figure A6. 70 Choice at $10 \mathrm{~km}$ in 2008 261

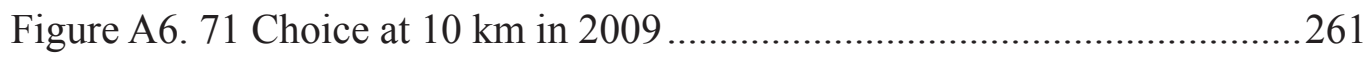

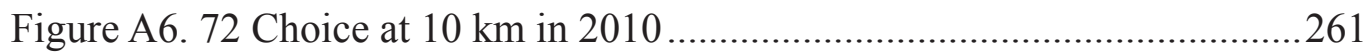

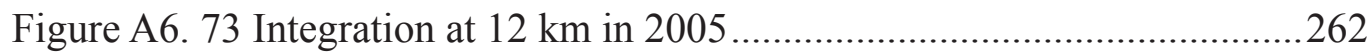

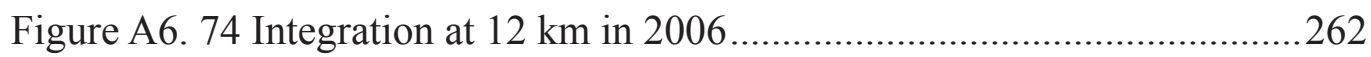

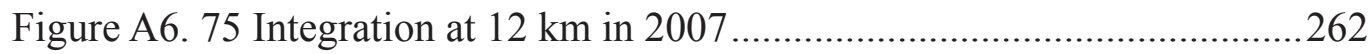

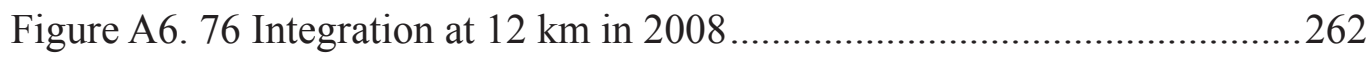

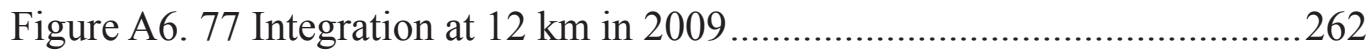

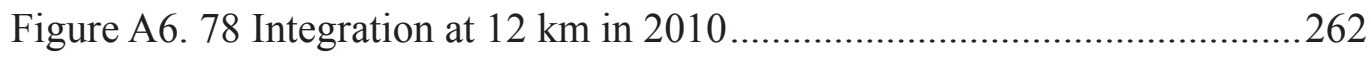

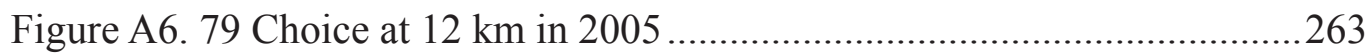

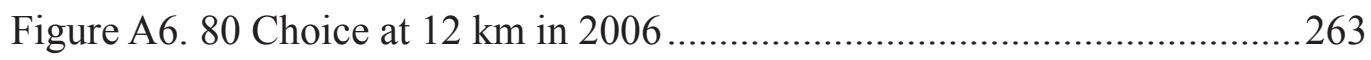

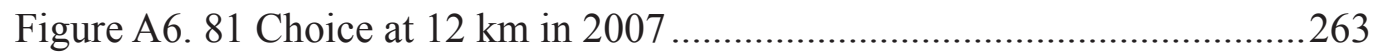

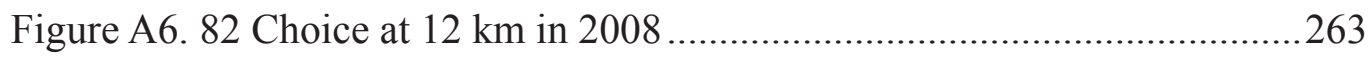

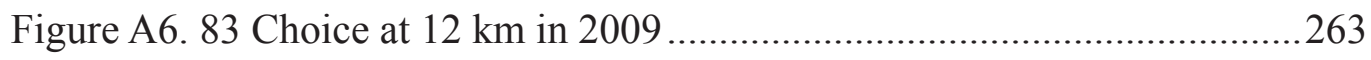

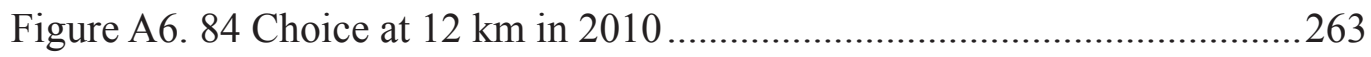

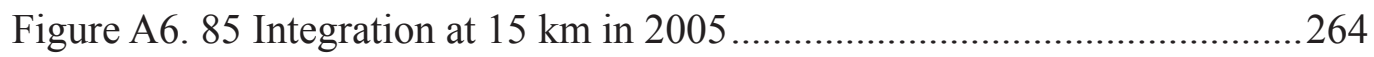




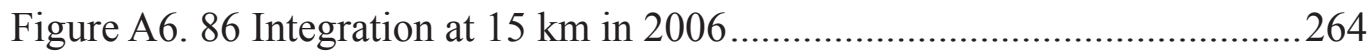

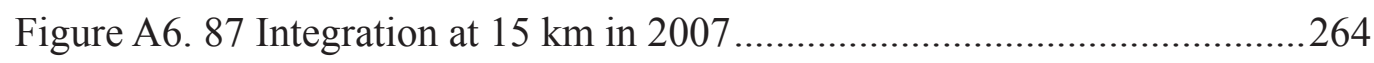

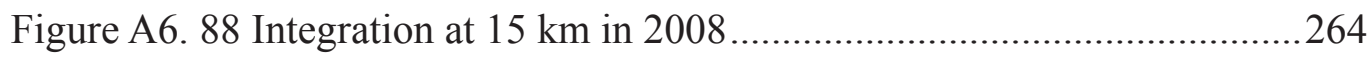

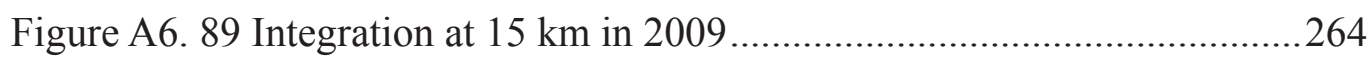

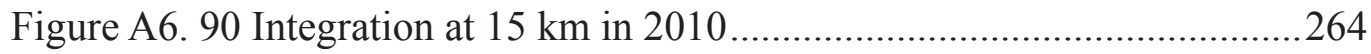

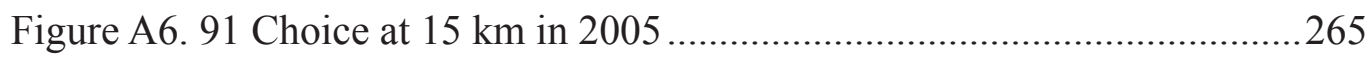

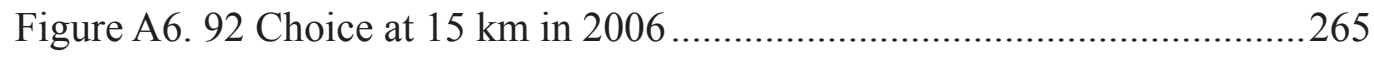

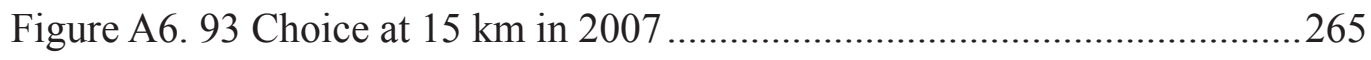

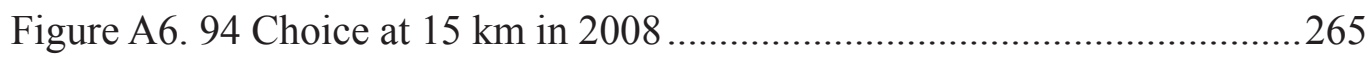

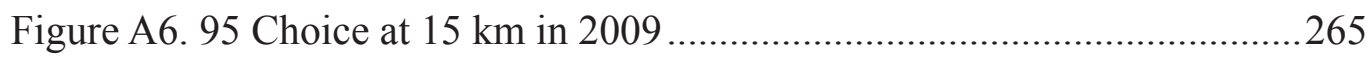

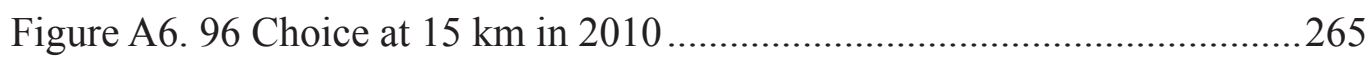

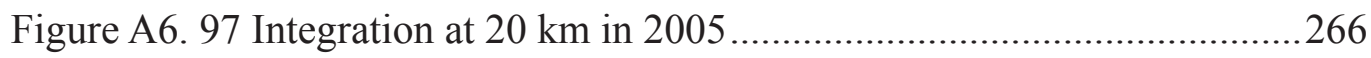

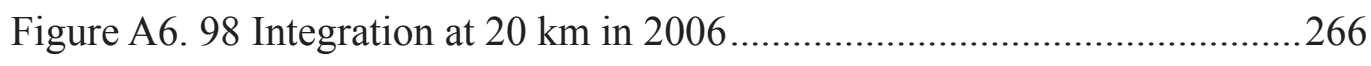

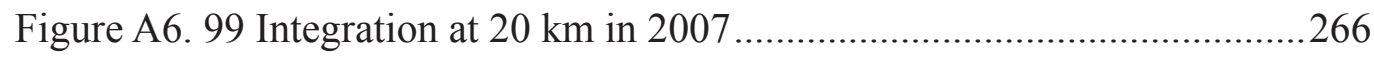

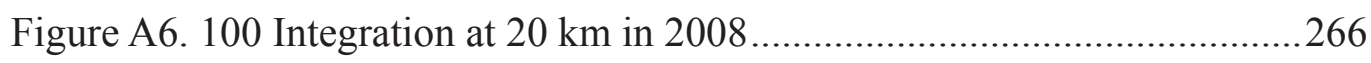

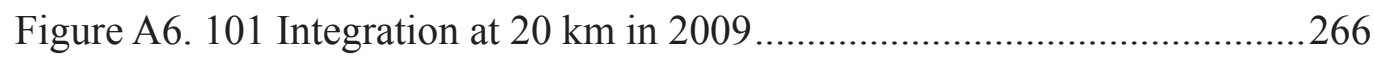

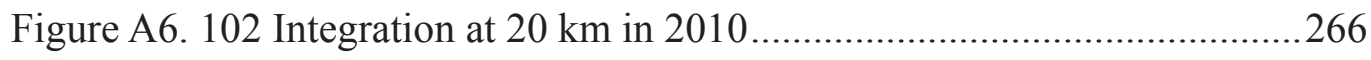

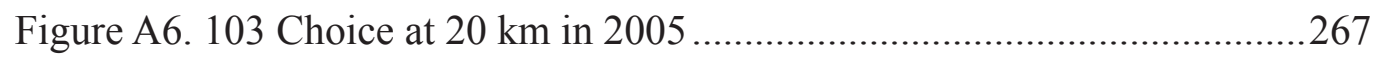

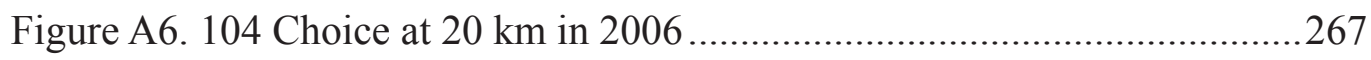

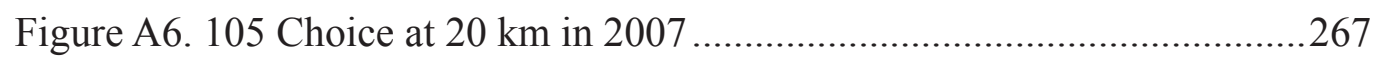

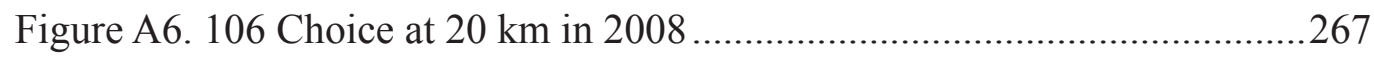

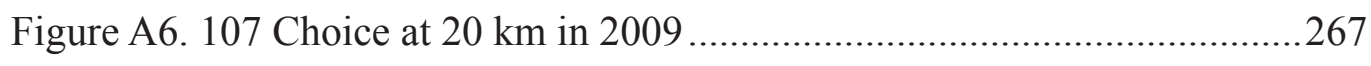

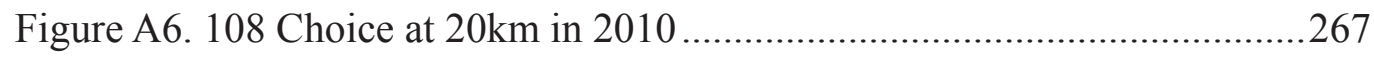

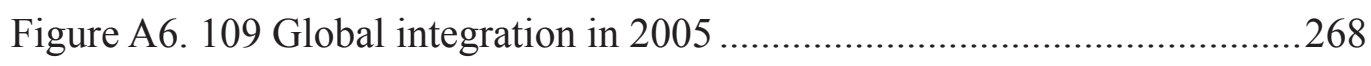

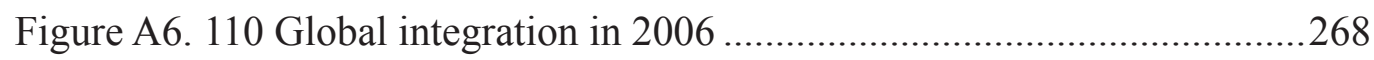

Figure A6. 111 Global integration in 2007 ..................................................268

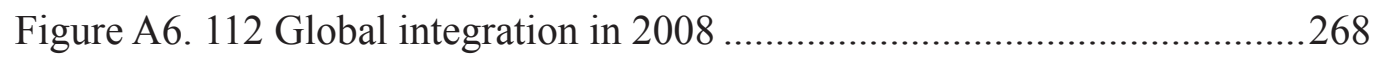

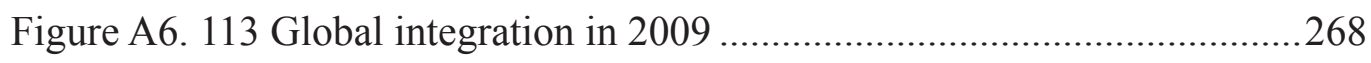

Figure A6. 114 Global integration in 2010 .................................................268 
Figure A6. 115 Global choice in 2005 269

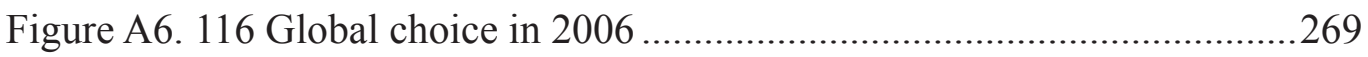

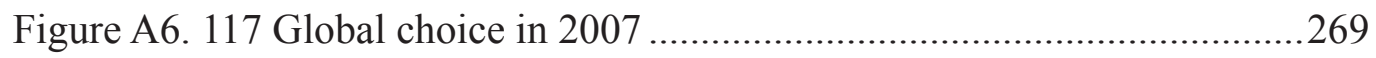

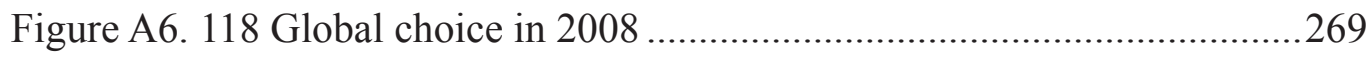

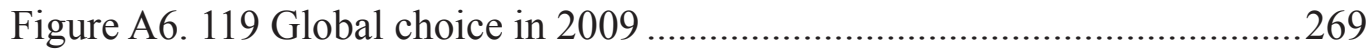

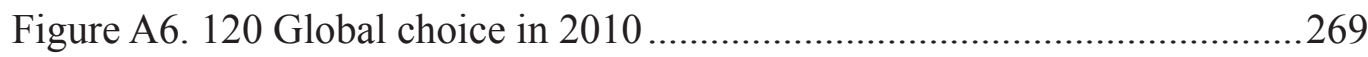




\section{Chapter One:}

\section{Introduction}

"We shape our buildings, and afterwards our buildings shape us." -----Winston Churchill

\subsection{Background}

Over the years, numerous conceptual, theoretical and empirical studies have attempted to formulate, model and quantify how the built environment is valued by people. However, studies of the valuation of urban morphology are rare, due to the lack of a powerful methodology to quantify the urban form accurately. In addition, neo-classical economic theories have emphasized location in respect to the city centre as the major spatial determinant of land value; but this has become weaker or even insignificant according to the findings of some current studies of mega cities, such as Los Angeles (Heikkila et al. 1989). Urban street networks contain spatial information on the arrangement of spaces, land use, building density, and patterns of movement and therefore give each location (or street segment) in the city a value in terms of accessibility. Thus, people can be thought of as paying for certain characteristics of the accessibility of the location of their choice. Moreover, they are likely to pay different amounts of money according to different demand levels.

The main motivation in this thesis is to investigate how urban morphology is valued. This is done through estimating its impact on the urban housing market, using the method of hedonic pricing. More specifically, the aim of this thesis is to examine whether street layout as an element of the urban form can provide extra spatial information in explaining the variance of housing price in a city, using both static and dynamic models. 
It is well known that commodity goods are heterogeneous, but that the unit of certain attributes or characteristics of the commodity good is treated as homogeneous (Lancaster 1966). Thus, people buy and consume residential properties as a bundle of "housing characteristics", such as location, neighborhood and environmental characteristics. Hedonic analysis studies the marginal price people willing to pay for characteristics of that product. Rosen (1974b) pointed out that in theory in an equilibrium market, the implicit price estimated by a hedonic model is equal to the price per unit of a characteristic of the housing property that people are willing to pay. There are many studies that have followed Rosen's approach in order to identify and value the characteristics that have an impact on housing price, including structural, locational, neighborhood and environmental characteristics (see for instance Sheppard, 1999; Orford, 2000; 2002).

Hedonic price models are widely used for property appraisal and property tax assessment purposes, as well as to construct house price indices. Furthermore, hedonic price models can be used for explanatory purposes (e.g. to identify the housing price premium associated with a particular neighborhood or design feature); and for policy evaluation or simulation purposes (e.g. to explore how the location of a new transit train might affect the property value; or whether the price premium associated with a remodeled kitchen will exceed the remodeling cost).

Orford (2002) notes that many hedonic studies are built upon the monocentric model of Alonso (1964) and Evans(1985), which underlined the importance of CBD as the major influence of land value and in which a bid-rent curve is translated into a negative house price curve (distance decay). Furthermore, in the early urban housing literature, the property value is differentiated based on its location and different sized units of homegenous housing units in a single market (Goodman and Thibodeau 1998). Thus, locational attributes (as the major determinant of land value) were the most important measure of hedonic housing price models. However, the monocentric 
model has inherent limitations and has increasingly been criticized by researchers as both an overly simplistic modeling abstraction and an empirically historical phenomenon (e.g. Boarnet, 1994). The monocentric model excludes non-transportation factors, for instance in cases where persons do not choose their residential location based on the wish to minimize their commuting costs to their work place. Moreover, when metropolitan areas are in a state of restructuring, and suburban employment centers exist, numerous studies have shown that the impact of distance to CBD becomes weaker, unstable or even insignificant (Heikkila et al. 1989; Richardson et al. 1990; Adair et al. 2000). Cheshire and Sheppard (1997) also argued that much of the data used in hedonic analyses still lack land and location information. Moreover, hedonic modeling studies ignore the potentially rich source of information in a city's road grid pattern. In order to understand people's preferences for different locations, urban morphology seems to have the potential of a theoretical and methodological breakthrough, since it has the ability to capture numerically and mathematically both the form and the process of human settlements.

With regards to the study of urban morphology, frequently referred to as urban form, urban landscape and townscape, it grows and shapes in the later of the nineteenth century, and is characterized by a number of different perspectives, such as those taken by geography and architecture (Sima and Zhang 2009). The studies of urban form in Britain have been heavily influenced by M.R.G. Conzen. The Conzenian approach is more interested in the description, classification and exemplification of the characteristics of present townscapes based on survey results; an approach that could be termed as an "indigenous British geographical tradition ". Later, this tended to shift from metrological analyses of plots to a wider plan-analysis (Sheppard 1974; Slater 1981).Recently the urban morphologists have come to examine the individuals, organizations and the process involved in shaping a particular element of urban form (Larkham 2006). In contrast, European traditions (e.g. Muratori1959,1963) take an architectural approach, stressing that elements, structures of elements, organism of 
structures are the components of urban form, which can also be called 'procedural typology'(Moudon 1997).

However, studies of urban morphology from the perspective of both geographers and urban economist are mainly interested in how and why individual households and businesses prefer certain locations, and how those individual decisions add up to a consistent spatial pattern of land uses, personal and business transaction, and travel behavior. For example, Hurd (1903) first highlighted land-value is not homogenous on topography on the street layout. He argued that one of advantage of irregular street layout is to protect central growth rather than axial growth, which allows people quick access to or from the business center. A rectangular street layout permits free movement throughout a city, and the effect will be promoted by the addition of long diagonal streets. In his study, Washington as a political city in US. provides an typical example of diagonal streets, where the large proportion of space are taken up by streets and squares, while it is not a mode for a business city. Another contribution Hurd made is mapping the price per frontage foot of a ground plan for several cities in US., showing the scale of average value (width and depth), see the example of Seattle showed in figure (1.1). Although he explained that the ground rent is a premium paid solely for location and all rent is based on the location's utility, the questions that why the high rental price located along linear as a axis, why there is bigger differentness of rental price despite how the streets approach to each other in the same area, and how to control the scale effects are not addressed. 


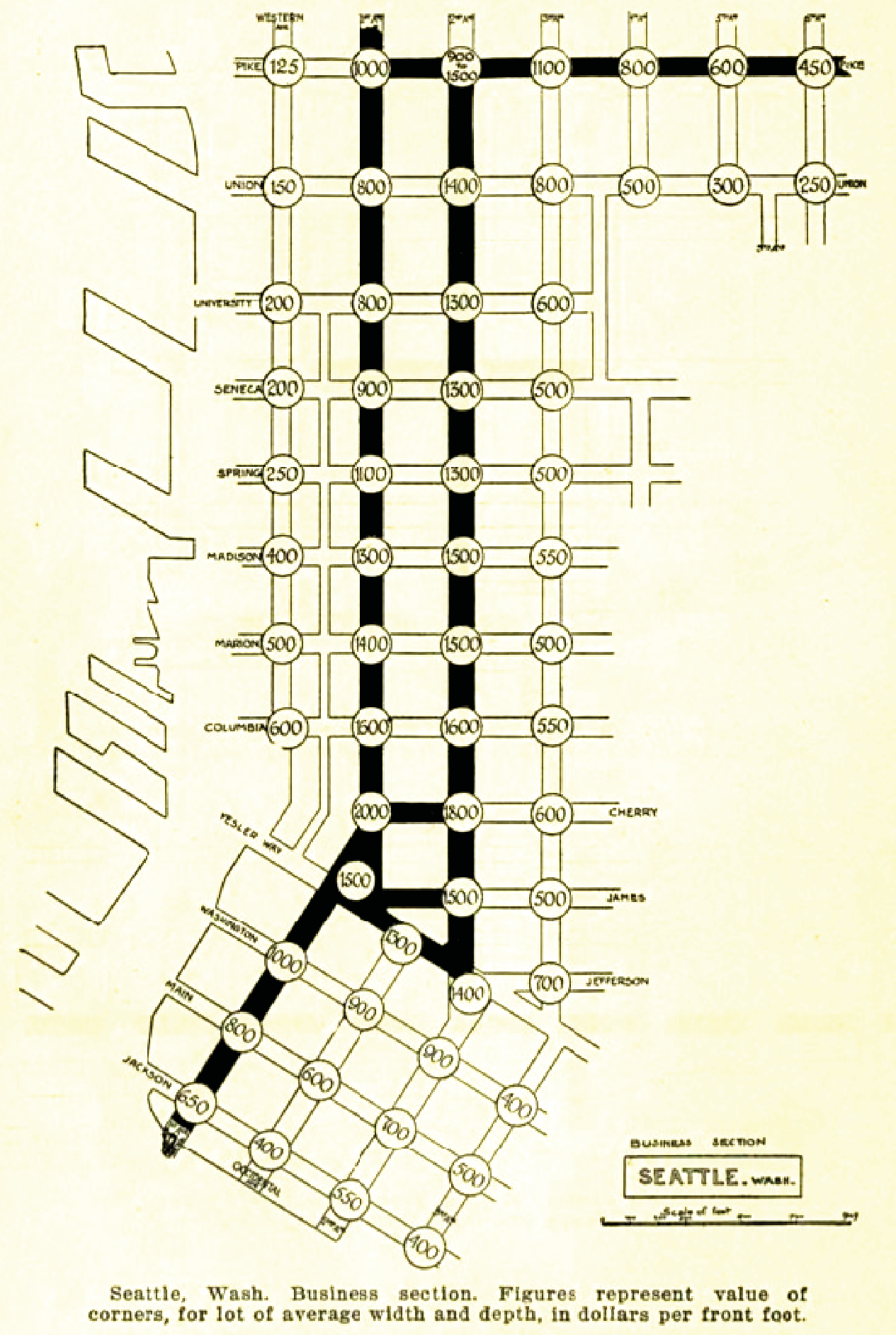

Figure 1.1Rent price pattern in Seattle

Source: Hurd (1903)

Webster (2010) takes an economist's approach and has pointed out several important issues that Hurd did not address. Street layout as the most essential element of urban 
form provides a basic geometry for accessibility, determining how street segments arrange possibilities and patterns of movement and transactional opportunities through 'spatial configuration'. The network gives each location (or street segment) in the city a particular connectivity value, and each part of the city, each road, each plot of land and each building has its own value as a point of access to other places, people and organizations. The general (connectivity to everywhere else) value of any point in the grid is also a profoundly significant economic value signifying access to opportunities for cooperative acts of exchange between one specialist skill and all others within the urban economy. Put another way, the street grid shapes the cost of transactions between an urban labour force: it spatially allocates the economy's division of labour. Thus, the geometric accessibility created by an urban grid is the most fundamental of all urban public goods. This being so, if it could be priced, it may be possible to allocate accessibility more efficiently. Measuring network-derived accessibility is the first step in so doing. It also allows for greater efficiencies in the design and planning of cities by governments and private developers when they build new infrastructure.

In spite of the crucial role of urban morphology to the urban economy, morphological studies are not a part of the mainstream planning literature, it seems, because verbal descriptions of properties cannot easily be translated into geometric abstractions and theories. In other words, it is lack of a sound scientific methodology for quantifying the urban form coherently. Early attempts were limited by the availability of software and hardware that could operate standard statistical approaches such as cluster analysis in order to research aspects of urban form (Openshaw 1973). The problems of establishing standard definitions in urban morphology and the perception that much of the information on urban form is not readily converted into 'data' has hindered the large-scale use of computers in storing and processing information. Alexander (1964, 1965) first introduced formal mathematical concepts into the debate in 1964. 
A range of early works in formal urban morphology explored how mathematical formalism such as graph theory and set theory could work in the urban design arena (e.g. March and Steadman 1971, Martin and March 1972, Steadman 1983). By the end of twentieth century, one innovative system of theories and techniques had emerged; known as 'Space Syntax'. It is an approach to urban form quite different from the British geographical tradition.

Space syntax originated as a quantified approach for spatial representation, which id developed in the 1970s at University College London. It is as a scientific and systematic way to study the interaction of people's movement and building environment. In book of 'The Social Logic of Space', Hillier and Hanson (1984b) noted that the exploration of spatial layout or structure has great impact on human social activities. Recently, the approach has been refined by Hillier (1996), Penn (2003), and Hillier and Penn (2004), with particular focus on the arrangement of spaces and possibilities and patterns of movement through 'spatial configuration'. Over the past two decades, space syntax theory has provided computational support for the development of urban morphological studies, revealing the characteristics of spaces in terms of movement and potential use. Space syntax has attempted to define the elements of urban form by measuring geometric accessibility; measuring the relationships between street segments by a series of measurements, such as connectivity, control, closeness and betweenness (Jiang and Claramunt 2002).

This thesis extends this tradition by employing space syntax methodology to refine hedonic price modeling. By so doing, it attempts to make a significant contribution to urban scholarship by exploring how finely measured urban morphology is associated with a number of housing market issues. In particular, I conduct a number of statistical experiments to find out how much people are willing to pay for different urban morphological attributes; or put another way, for different kinds of accessibility 


\subsection{Research questions}

This dissertation addresses three research questions relevant with urban morphology and housing markets.

The first question has three aspects: (a) whether the accessibility information contained in an urban configuration network model has a positive or negative impact on housing price; (b) assuming such relationships exist, whether the network model determinants of urban morphology are stronger or weaker than traditional locational attributes (such as the distance to CBD); (c) whether the relationship is constant in both disaggregated and aggregated levels.

The monocentric urban economic model and polycentric variants emphasize location, hypothesizing that house prices decrease with a growing distance to the $\mathrm{CBD}$, but more recent studies show that distance to CBD has become less important or even insignificant, suggesting either that people no longer choose their residential location based on minimum travel cost to work or that work has significantly dispersed within cities. Non-transportation factors (e.g. the distance to amenity and school quality), have become more influential in residential locations (White 1988a; Small and Song 1992).Therefore, many scholars attempt to explore the variety of preferences for location (e.g. the distance to a bus stop and distance to a park). However, these studies need a priori specification within a pre-defined area, identifying local attractions significant enough to influence locational choice systematically and measuring the proximity of the property to these attractive places.

However, this could cause econometric bias in the estimation, such as multicollinearity, spatial autocorrelation and omitting variables. The notion of general, systemic accessibility has been proven to better capture location options than the purely Euclidean distance in many studies on property value (e.g. Hoch and Waddell, 
1993), as it indicates the ability of individuals to travel more generally and to participate in various kinds of activities at different locations (Des Rosiers et al. 2000). However, accessibility indicators measuring attractiveness or proximity to an opportunity are normally applied to studies at an aggregated level (e.g. Srour et al., 2002), and disaggregated level accessibility measures still tend to rely on Euclidean distance or time cost from a location to particular facilities.

The accessibility information contained in an urban street layout model would seem, in principle, a suitable approach for measuring locational characteristics at a disaggregated level without a pre-defined map of or knowledge about attractiveness hot spots. This dissertation explores this proposition and thus contributes to this important theoretical and methodological gap in the hedonic house-price modeling literature.

The second question deals with the identification of housing submarkets by urban configurational features; and comparing this approach with traditional specifications of housing submarkets, asks whether network-based specifications produce efficient estimation results. It is known that housing submarkets are important, and people's demand for particular attributes vary across space. But within submarkets, the price of housing (per unit of service) is assumed to be constant. Generally, there are two mainstream schools of thoughts for identifying submarkets: spatial specification and non-spatial specification. Spatial specification stresses a pre-defined geographic area within which people's choice preferences are assumed to be homogeneous. This is criticized for being arbitrary. In contrast, non-spatial specification methods emphasize accuracy of estimation, advocating a data driven approach, which is criticized for being unstable over time (e.g. Bourassa et al.1999). These specifications for housing submarket are widely accepted in academic and practitioner fields in most developed countries with mature urban land markets. There is less knowledge about how to delineate sub markets in property markets of developing countries, where the building 
type in many fast growing cities is dominantly simplex (apartments) and social neighborhood characteristics are not long established and change quickly over time. This is the case in most cities in China.

This question contributes to another important gap in existing knowledge, as urban configuration features are assumed to be associated with both spatial information and people's preference. A network-based method could provide a new alternative specification for housing submarket delimitation that extends the non-spatial method by adding more emphasis on people's choice of location indirectly. The method could also help urban planners and government officials understand how different social economic classes respond to the accessibility of each location.

The third question has three aspects: (a) exploring micro-dynamic effects of urban configuration on housing price volatility; (b) asking whether this relationship is dynamic and synchronous over both space and time and whether submarkets exist as a result of this dynamic relationship; and (c)asking what kind street network improvements produce positive and negative spillover effects captured in property values.

The literature shows that most empirical analyses of house price movement focus on exploring the macro determinants of price movements over time using aggregate data, such as GDP, inflation indices and mortgage rates. Although some scholars state that accessibility could be a potential geographical determinant of house price volatility at a regional or city scale, there is little evidence confirming this relationship statistically. One reason for that is inaccurate measurements of accessibility(Iacono and Levinson 2011). In particular, it has proven difficult to measure changes inaccessibility at the disaggregated level, which is more reliant on Euclidean distance measures of accessibility. The premise of the research presented in this thesis, particularly in the chapter on China, hypothesizes that the continuous changes in urban street network 
that are associated with urban growth and the attendant changes in accessibility, are partial determinants of micro-level house price volatility. This question is particularly relevant in China, where the profound institutional reforms of urban housing systems and breathtaking urban expansion, have meant numerous investments into road network developments aimed at the urban fringe in order to facilitate the rapid expansion of cities. The city of Nanjing, used as a case study in Chapter Six is a good example, providing an opportunity to empirically examine the dynamic relationship between housing price and urban configurational change.

The findings of this dissertation should be of great value to urban planners and government officials in addressing the problem of managing urban growth efficiently, understand the multi-scale positive and negative externalities of road networks as captured in housing markets, assisting property value assessment for tax purposes, and evaluating urban land use policies and planning regulations.

\subsection{Thesis structures}

This thesis is organized into seven chapters.

After the introduction, chapter two investigates the literature on house price evaluation using the Hedonic price model. The approach covers several aspects, including the fundamental theory, theoretical criticisms, issues of estimation bias, and choice of housing attributes. In particular, the chapter focuses on the specification of the hedonic house price function form, housing submarkets and the debates on locational characteristics.

Chapter three provides a literature review of the methodology of space syntax-style network analysis. The basic notion of the space syntax method and the algorithms of two types of accessibility indices (integration and choice) areintroduced, respectively. 
Then, some key criticisms of space syntax are summarised. Finally, the chapter reviews empirical evidence on how urban morphology interacts with socio-economic phenomenon.

Chapters of four to six present theoretical and empirical analysis, which addresses the thesis' three research questions, respectively. In order to clearly delineate the theoretical contribution of each question, separate specific literature reviews are provided in each chapter.

Using a semi-log hedonic price functional form, chapter four adopts a part of the metropolitan area of Cardiff, UK as a case study to examining whether urban configurational features can impact the property value at both individual and output area level.

Chapter five uses the same Cardiff dataset, examining whether urban configurational features can be considered as an efficient specification alternative for identifying housing submarkets, especially when there is no predefined spatial boundary. Two-step clustering analysis is discussed in chapter and the results of a network approach to housing market delineation are compared to the results of two traditional approaches.

Chapter six setup a panel study of multi-year house prices to examine whether the continuous changes in urban street network associated with urban growth and the attendant changes in accessibility are partial determinants of micro-level house price volatility. This chapter uses the case of Nanjing, China in the time period from 2005 to 2010.The Space syntax method is employed in this chapter to track changes in accessibility within the urban street layout over time.

Finally, chapter seven presents the conclusions from the research. It also summarises 
the discussions of the three empirical chapters and presents brief reflections on the policy implications of the results. The chapter ends with comments on the limitations of the experiments presented in the thesis and with recommendations for future studies in this field. 


\section{Chapter Two:}

\section{Hedonic housing price theory review}

\subsection{Introduction}

The most commonly applied methods of housing price evaluation can be broadly divided into two groups: traditional and advanced methods. There are five traditional mainstream standard recognized valuation methods in the field of property valuation: comparative method (comparison), contractor's method (cost method), residual method (development method), profits method (accounts method), investment method (capitalization/income method).Advanced methods include techniques such as hedonic price modeling, artificial neural networks (ANN), case-based reasoning and spatial analysis methods.

Hedonic price modeling is the most commonly applied of these. Many scholars (e.g. Griliches, 1961) have referred to the work of Court (1939) as an early pioneer in applying this technique. He used the term hedonic to analyze price and demand for the individual sources of pleasure, which could be considered as attributes combined to form heterogeneous commodities. It was an important early application of multivariate statistical techniques to economics.

In this chapter, several aspects of hedonic modeling will be investigated in-depth, including the theoretical basis, the theoretical criticism, estimation criticism, and its use in pricing housing attributes, including accessibility (the subject of this thesis). Accordingly, the conclusion will mainly focus on the theoretical aspects of hedonic price modeling that are relevant to the question of which function form to choose in this study. 


\subsection{Hedonic model:}

In regards to the theoretical foundations, the hedonic model is based on Lancaster's (1966) theory of consumer's demand. He recognized a composite good whose units are homogeneous, such that the utilities are not based on the goods themselves but instead the individual "characteristics" of a good - its composite attributes. Thus, the consumers make their purchasing decision based on the number of characteristics a good as well as per unit cost of each characteristic. For example, when people choose a car, they would consider the quantity of characteristics from a car, such as fast acceleration, enhanced safety, attractive styling, increased prestige, and so on.

Although Lancaster was the first to discuss hedonic utility, he says nothing about pricing models. Rosen (1974a) was the first to present a theory of hedonic pricing. Rosen argue that an item can be valued by its characteristics, in that case, an item's total price can be considered as sum of price of each homogeneous attributes, and each attribute has a unique implicit price in a equilibrium market. This implies that an item's price can be regressed on the characteristics to determine the way in which each characteristic uniquely contributes to the overall composite unit price.

As Rothenberg et al. (1991) describes, the hedonic approach has two significant advantages over alternative methods of measuring quality and defining commodities in housing markets. First, compressing the many characteristics of housing into one dimension allows the use of a homogenous commodity assumption; and thus, the hedonic construction avoids the complications and intractability of multi-commodity models. Furthermore, the hedonic approach reflects the marginal tradeoffs that both supplier and demanders make among characteristics in the markets, so that differences in amounts of particular components will be given the weights implicitly prevailing in the market place. 


\subsubsection{Theoretical basis}

Housing constitutes a product class differentiated by characteristics such as number of rooms and size of lot. Freeman III (1979b) argued that the housing value can be considered a function of its characteristics, such as structure, neighborhood, and environmental characteristics. Therefore, the price function of house $h i$ can be demonstrated as

$$
P_{h i}=P_{h}\left(S_{i 1}, \ldots, S_{i j}, \ldots, N_{i 1} \ldots, N_{i k} \ldots, Q_{i 1} \ldots, Q_{i m}\right)
$$

Where:

The $S_{j}, N_{k}$ and $Q_{m}$ indicate the vectors of site, neighborhood, and environmental characteristics respectively.

Empirical estimation of Equation (2.1) involves applying one of a number of statistical modeling techniques to explain the variation in sales price as a function of property characteristics. Let X represent the full set of property characteristics $\left(S_{j}\right.$, $N_{k}$ and $\left.Q_{m}\right)$ included in the empirical model. The empirical representation of the $i$ th housing price is:

$$
\mathrm{p}_{\mathrm{i}}=\mathrm{p}\left(\mathrm{X}_{\mathrm{i}}, \beta, \varepsilon\right)
$$

Where

$\beta$ is a vector of parameters to be estimate

عis a stochastic residual term

$\mathrm{p}_{\mathrm{i}}$ is the implicit price respected to that characteristics 
Such as hedonic price models aim at estimating implicit price for each attributes of a good, and a property could be considered as a bunch of attributes or services, which are mainly divided into structural, neighborhood, accessibility attributes and etc. Individual buyers and renters, for instance, try to maximize their expected utility, which are subject to various constraints, like their money and time.

Freeman (1979) explains that a household maximizes its utility by simultaneously moving along each marginal price schedule, where the marginal price of a household's willingness to pay for an unit of each characteristic should equal to the marginal implicit price of that housing attribute. This clearly locates the technique within a neo-classical economics framework - a framework that analytically computes prices on the assumption that markets equilibrate under an 'invisible hand' with perfect information and no transaction costs. It is noted that although the theory of hedonics has been developed with this limiting theoretical context discussed above, the technique is typically applied as an econometric empirical model and does not rely on the utility maximization underlying theory.

To understand if a household is in equilibrium, the marginal implicit price associated with the chosen housing bundle is assumed equal to the corresponding marginal willingness to pay for those attributes. To unpack this, I begin with considering how a market for heterogeneous goods can be expected to function, and what type of equilibrium we can expect to observe. 

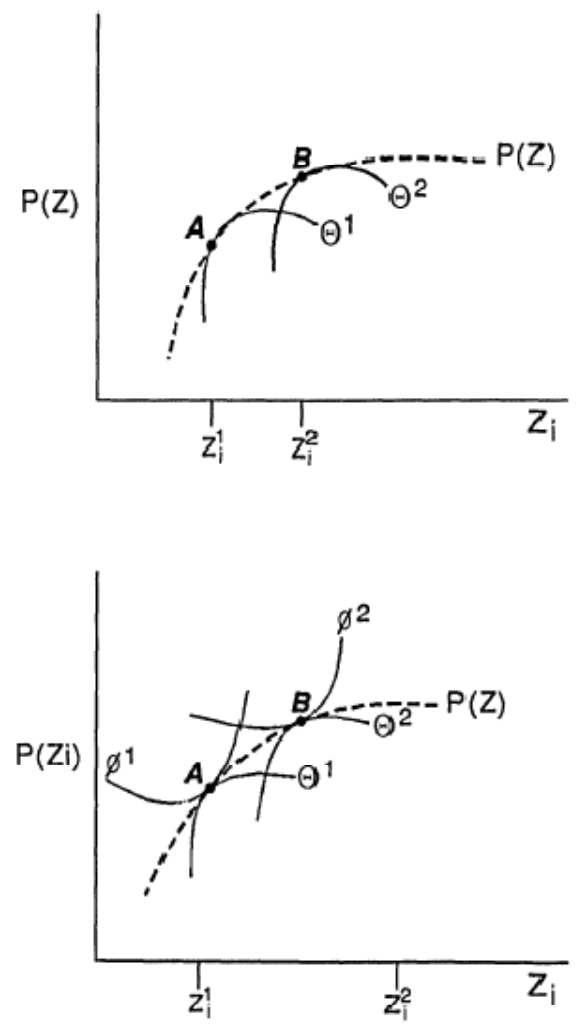

Figure 2.1 Demand and offer curves of hedonic price function

Source: Follain and Jimenez, 1985; pp.79

Following Follain and Jimenez's works (1985), a utility function can interpret a household decision, $\mathrm{u}(\mathrm{x}, \mathrm{z})$, where $\mathrm{x}$ is a composite commodity whose price is unity, and $\mathrm{z}$ is the vector of housing attributes. Assume that households want to maximize utility subject but with the budget constraint $y=p(z)+x$, where $y$ is the annual household income. The partial derivative of the utility function with respect to a housing attribute is the household's marginal willingness to pay function for that attribute. A first order solution requires $\frac{u_{z i}}{u_{x}}=p_{i}=\frac{\partial p(z)}{\partial\left(z_{i}\right)}, i=1, \ldots, n$, under the usual properties of $u$.

An important part of the Rosen model is the bid-rent function: 


$$
\theta\left(z_{i}, u, y, a\right)
$$

Equation (2.3)

Where $a$ is a parameter that differs from household to household.

This can be characterized as the trade-off a household is willing to make between alternative quantities of a particular attribute at a given income and utility level, whilst remaining indifferent to the overall composition of consumption.

$$
u=u(y-\theta, z, a)
$$

$\theta^{1}$ pictured in the upper panel of fig.(2.2) show that when solving the schedule for $\theta$. $\theta^{1}$ represented by households is everywhere indifferent along $\theta^{1}$ and $\theta$ schedules that are lower, which depend on its higher utility levels. It can be shown that

$$
\theta_{i}=\frac{u_{z}}{u_{x}}
$$

which is the additional expenditure a consumer's willingness to pay for another unit of $z_{i}$ and beequally well off (i.e. the demand curve). Figure 2.2 denotes two such equilibria: a for household $\theta^{1}$ and $\mathbf{B}$ for household $\theta^{2}$. 

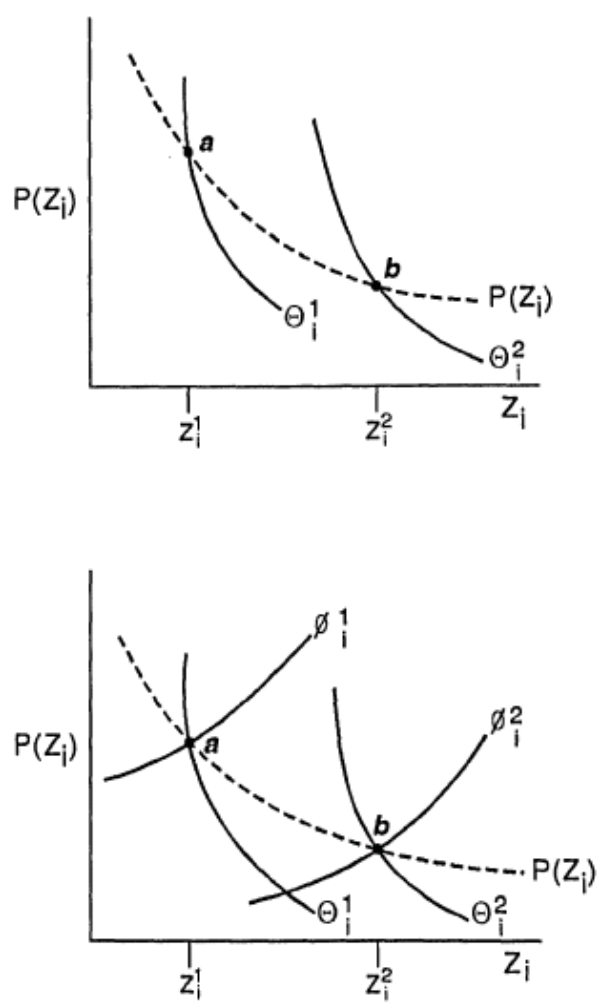

Figure 2.2 The marginal implicit price of an attribute as a function of supply and demand Source: Follain and Jimenez, 1985; pp.79

The supply side could also be considered, as $\mathrm{p}(\mathrm{z})$ is determined by the market,. When $\mathrm{P}(\mathrm{Z})$ as given, and constant returns to scale are assumed, each firm's costs per unit are assumed to be convex and can be denoted as $c(z, \beta)$, where the $\beta$ denotes factor price and production-function parameters. The firm then maximizes profits per unit $\pi=p(z)-c(z, \beta)$, which would yield the condition that the additional cost of providing that $i$ th characteristics, $C_{i}$, is equal to the revenue that can be gained, so that $p_{i}=c_{i}$.

Rosen (1974) emphasized that in fact the function $P_{h}$ is determined by a market in a clearing condition, where the amount of commodities offered by sellers at every point must equal to amounts demanded by consumers choosing. Both consumers and 
producer base their locational and quantity decisions on maximizing behavior and equilibrium prices are determined so that buyers and sellers can be perfectly scheduler. Generally, a market-clearing price are determined by the distributions of consumer tastes as well as producer costs.

However, Rosen did not formally present a functional form for the hedonic price function, his model clearly implies a nonlinear pricing structure.

\subsubsection{Hedonic price criticism}

One of the most important assumptions to come under attack is the one relating to perfect equilibrium. For this assumption to hold, it requires perfect information and zero transaction costs (Maddison 2001). If the equilibrium condition does not hold, the implicit prices derived from hedonic analysis are biased, because there is no $a$ priori reason to suppose that the extent of disequilibrium in any area is correlated with the levels if particular amenities contributing to the hedonic house price. The consequence of disequilibrium is likely to be in increased variance in results rather systematic bias (Freeman III 1993). Furthermore, Bartik (1987) and Epple (1987) also point out that the hedonic estimation is not to the result of demand-supply interaction, as in the hedonic model, an individual consumer decision does not affect the hedonic price function, which implies that an individual consumer's decision cannot affect the suppliers.

Follain and Jimenez (1985) argue that the marginal price derived from the hedonic function does not actually measure a particular household is willing to pay for a unit of a certain characteristic. Rather, it is a valuation that is the result of demand and supply interactions in the entire market. Under the restrictive condition of homogeneous preferences - another limitation of the neo-classical model - the hedonic equation can reveal the underlying demand parameters for the representative 
household. When all households are similar with homogenous characteristics of income and socio-economic and supplies are different, the hedonic coefficient will be the marginal willingness to pay. Only in extreme cases when all consumers have identical incomes and utility functions will the marginal implicit price curve be identical to the inverse demand function for an attribute. With identical incomes and utility functions, these points all fall on the same marginal willingness to pay curve (Freeman 1979). Hence, the implicit price of an attribute is not strictly equal to the marginal willingness to pay, and hence demand for that attribute.

Another issue raised by Freeman (1979) is the speed of adjustment of the market to changing condition of supply and demand. If adjustment is not complete, observed marginal implicit price will not accurately measure household marginal willingness to pay. When the demand for an attribute is increasing, marginal implicit prices will underestimate true marginal willingness to pay. This is because marginal willingness to pay will not be translated into market transactions that affect marginal implicit price until the potential utility gains pass the threshold of transactions and moving cost.

Finally, the market for housing can be viewed as a stock-flow model where the flow is a function form, but the price at any point in time is determined only by the stock at that point in time. This raises a concern about the accuracy of the price data itself. Given that the data is based on assessments, appraisals, or self-reporting, it may not correspond to actual market price. The errors in measuring the dependent variable will tend to obscure any underlying relationship between true property value measures and environment amenities. But the estimation of the relationship will not be biased unless the errors themselves are correlated with other variables in the model. 


\subsubsection{Estimation criticism}

The hedonic price model relies on regression technology, which is criticized by some authors for a series of econometric problems that can lead to the bias of estimation, such as function specification, spatial heterogeneity, spatial autocorrelation, housing quality change, multicollinearity and heteroscedasticity.

\subsubsection{Function specification}

Hedonic models are sensitive to choice of functional form, as economic theory gives no clear guidelines on how to select the functional form. Rosen (1974a) demonstrated that the hedonic price functional form is a reduced form equation which reflect mechanisms of both supply and demand. A further important task facing researchers is how to function the relationships of dependent variable and the explanatory variables naturally, which impose an incorrect functional form on the regression equation will lead to misspecification bias. The simple approach is the ordinary linear approach, but if the true functional form of the hedonic equation is not linear, there will occur inconsistent estimation in the resulting coefficients (Linneman 1980). Freeman (1979) specified the Box-Cox transformation, which allows choice of the proper function form based on the structure of a particular data set. Typically, hedonic price regression models can be classified into four simple parametric functional forms;

a. Linear specification: both the dependent and explanatory variables enter the regression with linear form.

$$
\mathrm{p}=\beta_{0}+\sum_{\mathrm{k}=1}^{\mathrm{K}} \beta_{\mathrm{k}} \mathrm{x}_{\mathrm{k}}+\varepsilon
$$

Equation (2.6)

Where:

$\mathrm{p}$ denotes the property value.

$\varepsilon$ is a vector of random error term

$\beta_{\mathrm{k}}(\mathrm{k}=1, \ldots, \mathrm{K})$ indicates the marginal change of the unit price of the $\mathrm{k}^{\text {th }}$ 
characteristic $\mathrm{x}_{\mathrm{k}}$ of the good.

b. Semi-log specification: in a regression function, dependent variable is log form and explanatory variable is linear, or dependent variable is linear and explanatory variable is $\log$ form.

$$
\operatorname{Lnp}=\operatorname{Ln} \beta_{0}+\sum_{\mathrm{k}=1}^{\mathrm{K}} \beta_{\mathrm{k}} \mathrm{x}_{\mathrm{k}}+\varepsilon \text { Equation }
$$

Equation (2.7)

Where:

$\mathrm{p}$ denotes the property value.

cis a vector of random error term

$\beta_{\mathrm{k}}(\mathrm{k}=1, \ldots, \mathrm{K})$ indicates the rate at which the price increases at a certain level, given the characteristics $\mathrm{x}$

c. Log-log specification: in a regression function, both the dependent and explanatory variables are their log form.

$$
\operatorname{Lnp}=\operatorname{Ln} \beta_{0}+\sum_{\mathrm{k}=1}^{\mathrm{K}} \beta_{\mathrm{k}} \operatorname{Ln} \mathrm{x}_{\mathrm{k}}+\varepsilon \text { Equation }
$$

Equation (2.8)

Where:

$\mathrm{p}$ denotes the property value.

cis a vector of random error term

$\beta_{\mathrm{k}}(\mathrm{k}=1, \ldots, \mathrm{K})$ indicates how many percent the price $\mathrm{p}$ increases at a certain

level, if the $\mathrm{k}^{\text {th }}$ characteristic $\mathrm{x}_{\mathrm{k}}$ changes by one percent.

d. Box-Cox transform: determine the specific transformation from the data itself then enter the regression in individual transformed form.

$$
\mathrm{p}^{(\theta)}=\beta_{0}+\sum_{\mathrm{k}=1}^{\mathrm{K}} \beta_{\mathrm{k}} \mathrm{x}_{\mathrm{k}}{ }^{\left(\lambda_{\mathrm{k}}\right)}+\varepsilon
$$

Where:

$$
\begin{gathered}
\mathrm{p}^{(\theta)}=\frac{\mathrm{p}^{(\theta)}-1}{\theta}, \theta \neq 0 \\
=\operatorname{Lnp}, \theta=0 \\
\mathrm{x}^{\left(\lambda_{\mathrm{k}}\right)}=\frac{\mathrm{x}^{\left(\lambda_{\mathrm{k}}\right)}-1}{\lambda_{\mathrm{k}}}, \lambda_{\mathrm{k}} \neq 0 \\
=\operatorname{Lnx}_{\mathrm{k}}, \lambda_{\mathrm{k}}=0
\end{gathered}
$$


From the Box-Cox transform equation we can see if the $\theta$ and $\lambda_{\mathrm{k}}$ are equal to 1 , the model will transform to the basic linear form. If the $\theta$ and $\lambda_{\mathrm{k}}$ are equal to 0 , the model will transform to the $\log$-linear form. If the value $\theta$ is equal to 0 and $\lambda_{\mathrm{k}}$ are equal to 1 , then the model can be the semi-log form.

\subsubsection{Debate about the hedonic function}

Unfortunately, economic theory provides little guidance, and there is no specific function form for the hedonic price models suggested by Rosen (1974), Freeman (1979), Halverson and Pollakowski (1981) and Cassel and Mendelsohn (1985), so it is reasonable to try several functional forms to find the best performance. Among the four types of function forms in hedonic literatures, the semi-logarithmic form is much more prevalent, as it is easy to interpret its coefficients as the proportionate change in price arising from a unit change in the value of the characteristic. Furthermore, unlike log-log models, the semi-log model can deal with dummy variables for characteristics that are either present or absent (0 or 1). Diewert (2003) argued that the errors from a semi-log hedonic function are homoskedastic (have a constant variance).

Although more and more researchers prefer to use the Box-Cox transformation function, letting the dataset drive the function form, Cassel and Mendelsohn (1985) pointed out four inconsistencies of the Box-Cox transformation. Firstly, the large number of coefficients estimated with Box-Cox reduce the accuracy of any single coefficient, which could lead to poorer estimates of price. Secondly, the traditional Box-Cox functional form is not suited to any data set containing negative numbers. Furthermore, the Box-Cox function may be invalid for prediction, as the mean predicted value of the untransformed dependent variable need not equal the mean of the sample upon which is estimated. The predicted untransformed variables will be biased, and the predicted untransformed variables may also be imaginary. Fourth, the nonlinear transformation results in complex estimate of slopes and elasticities, which 
are often too cumbersome to use properly.

Taking least error as the choice criterion, Crooper et al. (1988) compared six function forms: linear, semi-log, double-log, Box-Cox linear, quadratic and quadratic Box-Cox, testing the best goodness of fit using data for Baltimore. His studies found that no function produced the lowest $\beta \mathrm{i}$ for all the attributes, although the quadratic Box-Cox function had the lowest normalized errors. However, the linear Box-Cox function had the lowest error variance, and based on the criterion, the linear Box-Cox performed the best and quadratic and double-log functions the worst. On the other hand, when variables are replaced or omitted, the Box-Cox linear function was the best of the six.

Having said all this, Halvorsen and Pollakowski (1981)rightfully pointed out that the true hedonic function form is unknown: we can only estimate it for any particular data set, although as I have shown, we do have methods to help choose the most appropriate parametric hedonic function form.

\subsubsection{Housing submarkets}

Housing property cannot be regarded as a homogeneous commodity. A unitary metropolitan housing market is unlikely ever to exist. Instead it is likely to be composed of interrelated submarkets (Adair et al. 1996; Tu 1997; Goodman and Thibodeau 1998; Whitehead 1999; Watkins 2001). Straszheim (1974) suggested that the housing market is a series of single markets, which requires different hedonic functions. According to Schnare and Stuyk (1976), housing submarkets arise when competition in a housing market is insufficient to ensure spatial equalization of physical housing attributes. Thus, the submarkets existence is the results of inelasticity (or high inelasticity) of demand and supply of housing at least in a short term. Bau and Thibodeau (1998) define housing sub-market as follows: 
"Housing submarket are typically defined as geographic area where the prices per unit of housing quantity (defined using some index of housing characteristic) are constant."(Bau and Thibodeau, 1998).

Goodman and Thibodeau (1998) argue that the existence of submarket questions the validity of the traditional assumption that urban housing markets can be modeled on the basis of a single market-wide house price equation. Adair et al. (1996), also argue that the failure to accommodate the existence of housing submarket will introduce bias and error into regression-based property valuation. Orford (2000), demonstrates that submarkets could be considered as relatively homogeneous sub-groups of the metropolitan housing market, people's the preference on each housing attribute may vary in different submarkets whilst remain the same within each submarket. However, the theory assumes that the implicit price for per unit of each housing attribute is stationary over space, and this assumption ignores that different geographical demand and supply characterized by different classes of people can lead to the spatial disequilibrium of housing market in a metropolitan area. Thus, parameters estimated by a simple hedonic function for the whole market sometimes seems misleading.

Goodman and Thibodeau (2007) emphasize that housing submarkets are important in house price modeling for several reasons. Firstly, the assigning of properties to housing submarkets is likely to increase the accuracy of the prediction of the statistical models, which are used to estimate house prices. Secondly, identifying housing submarket boundaries within metropolitan areas will increase the chance of researchers deriving better spatial and temporal variations in their models of prices. Thirdly, the accurate allocation of properties to submarkets will improve the abilities of lenders and investors to price the risk related to the financing of homeownership. Finally, the provision of submarket boundary information to housing consumers will decrease their search costs. 
In terms of the specification of housing submarkets, Goodman and Thibodeau (1998) stated that a metropolitan housing market might be segmented into groups of submarkets according to the factor of demand and / or supply. Watkins (2001) also suggests that housing submarkets exist as dwelling can generate different price due to the interaction between segmented demand characterized by consumer groups, and segmented supply characterized by product groups. As such, housing submarkets may be defined by dwelling type (e.g., town house, flat and detached house); by structural characteristics (numbers of bedroom, and building style); by neighborhood characteristics (e.g., school quality). Alternatively, housing markets may be segmented by age, income and race of households (Schnare and Struyk 1976; Gabriel and Wolch 1984a; Munro 1986; Allen et al. 1995). In that case, higher income households tend to be willing to pay more for housing (per unit of housing services)and the attributes of other home-owners - to protect the homogeneity of their neighborhood, life chances of children and so on. Finally, racial discrimination may produce separate housing submarkets for majority and minority households (King and Mieszkowski 1973). Several empirical studies of submarkets have, found that spatial characteristics are more important than structure characteristics. Ball and Kirwan (1977) found housing affordability and the availability of mortgage finance to be important shapers of sub markets, despite spatial constraints. Historical characteristics can also contribute to housing market segmentation. More recently, scholars have been more aware of the importance of both spatial and structural factors as the specification criterions of housing submarket (Adair et al. 1996; Maclennan and Tu 1996).

Although, many researchers agree on a sub-market definition based on structural and locational features, there is little consensus as to how a submarket should be identified in practice. The most common procedure for testing submarket existence was introduced by Schnare and Struyk (1976) and has been employed subsequently (for example, Dale-Johnson 1982, Munro 1986). The test procedure involves three stages. 
First, hedonic house price functions are estimated for each potential market segment in order to compare the submarket price for a 'standard' dwelling. Secondly, a chow test is computed in order to show whether there are significant differences between the submarket specific prices. Thirdly, a weighted standard error is calculated for the submarket model, which acts as a further 'common-sense' test of the significance of price differences for standard dwellings in different submarkets. This procedure also enables us to do a comparison of the effects on the accuracy of the house price models when different submarket definitions and stratification schemes are being compared.

Bourassa et al.(1999) stressed the need to test whether boundaries of submarkets are stable over time. Adding a dynamic part to the analysis makes it even more difficult to specify sub-market models since markets are constantly changing.

\subsubsection{Spatial autocorrelation}

A further discussion in terms of the application of hedonic price modeling is spatial dependency, also known as spatial autocorrelation. One of the basic assumptions underlying the regression model is that observations should be independent of one another. However, from the first law of geography, attributed to Tobler (1970), 'everything is related to everything else, but near things are more related than distant things', the independence of observations assumption is clearly a problem. Spatial autocorrelation is concerned with the degree to which objects or activities at some place in the earth's surface are similar to other objects or activities located nearby (Goodchild 1986). This is important in the sense that it is a special feature of spatial data (Can 1990); for example, houses that are close in geographic space are likely to have similar attributes. Generally, if the spatial effect is ignored, it is more likely that the real variance of the data is underestimated and thus leads to bias of the results (Ward and Gleditsch 2008). According to the works of Dunse et al. (1998), Bowen 
et al. (2001), Gillen et al. (2001), and Orford (1999), there are at least three sources of spatial autocorrelation, including property characteristics, the evaluation process; and mis-specification in the OLS model.

Firstly, spatial dependency exists because nearby properties, have similar property characteristics, in particular for structural features, as the properties were developed at the same time and also share the same locational conditions (Gillen et al. 2001; Bourassa et al. 2005).Secondly, spatial autocorrelation also arises from the valuation process, as the transaction price agreed between buyers and sellers will affect the price of the surrounding area (Bowen et al. 2001), especially where valuers use the comparison method, which is most common in the residential real estate industry. Thirdly, mis-specification of a model can result in spatial autocorrelation (Orford 1999), when the model is missing important variables, has extra unimportant variables, and / or an unsuitable functional form. Anselin (1988) also states that spatial autocorrelation is associated with spatial aggregation, the presence of uncontrolled-for non-linear relationships, and the omission of relevant variables.

Generally, spatial autocorrelation analysis is applied for testing whether the observed value of a variable is independent of the values of the variable with neighbors. The function of a spatial autocorrelation index is to measure the degree of interdependence between variables, and the strength and nature of that interdependence. It may be categorized as positive and negative, respectively. Positive autocorrelation occurs when high or low values of the random variable tend to cluster in space, whereas negative autocorrelation takes place when locations tend to be surrounded by neighbors with very dissimilar characteristics. Commonly, Moran's I test measures spatial dependency in the residuals of a regression model, and it checks the similarities among the housing price and attribute data in relation to the spatial relationships (Bowen et al. 2001).If there are $N$ observations on a variable $x$ at locations $i, j$, the formula for Moran's I is : 


$$
I=\frac{\left(N / S_{0}\right) \sum_{i} \sum_{j} w_{i j}\left(x_{i}-\mu\right)\left(x_{j}-\mu\right)}{\sum_{i}\left(x_{i}-\mu\right)^{2}}
$$

Where:

$\mu$ is the mean of the $x$ variable, $\mathrm{w}_{\mathrm{ij}}$ are the elements of the spatial weights matrix, $\mathrm{S}_{0}$ is the sum of the elements of the weights matrix.

The range of Moran's $I$ is from -1 to +1 , and an expected value (zero) shows absence of autocorrelation in samples. Moran's I compares the relation between the deviations from the mean across all neighbors of I, adjusted for the variation in $\mathrm{y}$ and the number of neighbors for each observation. Higher value of Moran's I indicate stronger positive clustering, which means the values from neighboring units are similar to one another.

\subsubsection{Housing quality change}

Although the hedonic model has been used for housing market analysis for more than 30 years, and most studies rely on one-shot studies of one place at one point in time (Richardson et al. 1990), the modeling approach has been criticized for its instability of the coefficients over time (Case and Quigley 1991; Quigley 1995; Case et al. 2006). According to the Dhrymes (1971) most studies consider the time variables as essential explanatory variables, which imply that they are a proxy for change in quality over time. Griliches (1996) constructed a hedonic price index for automobiles in an attempt to measure the change in quality over time, and he noticed the coefficient of the characteristics were unstable and changed over time. McMillen (2003) took an alternative repeat sales model to identify changes in house price distance gradients in Chicago. He employed the transaction data of Chicago from January 1, 1983 to December 31, 1998, and he found that the distance from the CBD did not affect house prices in the City of Chicago significantly in the early to mid 1980s. However, the situation reversed in the 1990s as a significant CBD house price gradient was 
reestablished, and by the end of the 1990 s, house values fell by more than $8 \%$ with each additional mile of distance from the CBD. He explained that this change in gradients was caused by a rapid appreciation of values near the city center as new housing was built, which increased demand for housing near Chicago's center.

Similarly, Hulten (2005) criticized the hedonic price method as failing to capture dynamics since price inflation and quality both change over time. Inflation could lead to an upward shift in the hedonic function because some or all characteristics become more expensive, whereas quality change can be caused by changes in compositions of housing attributes and by product innovation. Changes in compositions of varieties can occur with changes in people's income, tastes, demographics and environmental preferences. For example, opening a new train station will affect people's choice of location in respect to living near job centers. On the other hand, product innovation occurs when the cost of acquiring a number of characteristics is reduced. For example, in the past whether the property has a fireplace can affect the property value, but nowadays the fireplace is less significant as the construction cost for installing a fireplace is lower.

Some scholars attempt to explore the relationship between property value and certain attributes in temporal, such as the question of how soon the housing price will increase due to the kind of attribute adding the value. For example, Gatzlaff and Smith (1993) examined the impact of the development of the rail system on the residential property values in Miami. Using hedonic model, they found that the rail development announcement's impact on residential property value is weak. However, comparing a house price index for properties located near rail stations versus the housing price index for the Miami MSA indicates a weak relative increase in house value close to the station. Based on the results, they concluded that the Metro rail system has little effect on accessibility improvement. Noonan (2007) examined the effect of historic landmarks on property value in Chicago using a hybrid hedonic 
model and repeat-sales model. The results suggests that housing near landmark buildings sold at a small premium during the 1990s.

\subsubsection{Multicollinearity}

Another issue that researchers often encounter when they attempt to estimate the hedonic function is multicollinearity (Lake et al. 2000; Orford 2002). Multicollinearity is a statistical phenomenon when two above exploratory variables in a multiple regression model are highly correlated. For example, it is well known that both traffic noise and air pollution have a negative impact on housing properties, however, likely that traffic noise and air pollution are highly correlated, as high level traffic flows could result in poor air quality. In this case, regression analysis finds it difficult to tease apart the separate influence on property price, consequently the estimation for each parameter is no longer reliable. There is no easy solution to the problem of multicollinearity and the parameters estimated maybe implausibly large or have the wrong sign (e.g. the opposite relationship). Sometimes, it is possible to overcome multicollinearity by measuring the variables more accurately, or applying principal components analysis to combine the highly correlated variables into one index.

However, multicollinearity does not reduce the predictive power and reliability of whole model, at least within the sample size. It only affects calculation regarding individual predictors. There are several methods for detecting multicollinearity such as VIF, Condition Number test and Farrar-Glauber test. The studies in this thesis use the variance inflation factor (VIF) to measure the multicollinearity, and its formula is as following:

$$
V I F=\frac{1}{1-R_{j}^{2}}
$$


Where $\mathrm{R}_{\mathrm{j}}^{2} R_{j}^{2}$ is the coefficient of determination of a regression of explanatory $\mathrm{j}$ on all the other explicators. If VIF is more than 10, it indicates there is serious multicollinearity problem.

\subsubsection{Heteroscedasticity}

Heteroscedasticity is a phenomenon where the variance of the disturbance or error term of the hedonic model are unequal (Fletcher et al. 2000) or changes across the sample (Hendry 1995). One assumption of OLS is that the variance of the error should not be correlated with the dependent variables. Although it can happen in both time series and cross sectional data, it is more commonly present in studies employing cross-sectional data (Nghiep and Al 2001). According to Gujarati (2003), heteroscedasticity (constant variance) does not cause ordinary least squares coefficient estimates to be biased. The estimates are consistent but inefficient because their variances are no longer minimized, even if the sample size is increased. However, it could lead to unreliable confidence intervals, and $\mathrm{t}$ and $\mathrm{F}$ tests.

There are two main sources of heteroscedasticity; statistical methods of modeling and economic behavior of the sample (Hendry 1995). A common statistical source of error of measurement arises in the situation where data for a high level of geographical aggregation, such as census area data, are used to represent individual properties. An example of a behavioral source is the effect of different bargaining power of housing consumers. This can induce heteroscedasticity as the more the bargaining power of consumers, the more the possibility for the price to be lower (Harding et al. 2003).

Thus, heteroscedastic data could bias the estimations for the relationship between the predictor variable and the outcome. However, heteroscedasticity can be detected by series of diagnostic test, such as Park test, Glejsertest, Whitetest, Breusch-Pagan test, Goldfeld-Quandt test, Cook-Weisberg test and etc. In this study, the White test would 
be applied to detect whether there is heteroscedasticity in the error terms;

$$
\begin{gathered}
Y_{i}=\beta_{1}+\beta_{2} X_{i 2}+\beta_{3} X_{i 3}+u_{i} \\
u_{t}^{2}=\alpha_{1}+\alpha_{2} X_{i 2}+\alpha_{3} X_{i 3}+\alpha_{4} X_{i 2}^{2}+\alpha_{5} X_{i 2}^{2}+\alpha_{6} X_{i 2} X_{i 3}
\end{gathered}
$$

Step 1: using OLS procedure, obtain $\widehat{\beta_{1}}, \widehat{\beta_{2}}$ and $\widehat{\beta_{3}}$.

Step 2: $\quad$ Square the residual $\hat{u}_{i}^{2}=\left(Y_{i}-\widehat{\beta_{1}}-\widehat{\beta_{2}} X_{i 2}-\widehat{\beta_{3}} X_{i 3}\right)^{2}$

Step 3: Regress the squared residual $\hat{u}_{i}^{2}$ against a constant $X_{i 2}, X_{i 3}, X_{i 2}^{2}, X_{i 2}^{2}$ and $X_{i 2} X_{i 3}$,

Step 4: Compute the statistics $n R^{2}$ where the $n$ is the size of the sample and $\mathrm{R}^{2}$ is the unadjusted R-square from the step 3

Step 5: Reject the null hypothesis that $\alpha_{1}=\alpha_{2}=\alpha_{3}=\alpha_{4}=\alpha_{5}=\alpha_{6}=0$ if $\mathrm{nR}^{2}>$, $X_{5}^{2}(\alpha)$, the upper 5 percent point on the chi-square distribution with 5 d.f.

\subsection{Housing attributes}

The basic hypothesis of hedonic housing models is that housing price can be considered as willingness to pay for a bundle of characteristics. Empirical studies have generally grouped determining variables into three subsets:

a. Structural or internal attributes describe the physical characteristics of housing (e.g. numbers of bedroom, swimming pool, and garage).

b. Locational attributes include the distance to major places of employment, to major amenities (e.g. shopping mall and public facilities, etc.), and to road infrastructure and transport access points (e.g. train station, subway station, major streets, highways, airports, etc.).

c. Neighborhood attributes depict the quality of the economic and social 
characteristics of the neighborhood (e.g. income status and racial composition).

d. Environmental attributes describe environmental quality and environmental amenities, such as air pollution, water pollution, noise, aesthetic views and proximity to recreational sites or public service.

These are discussed in the following 


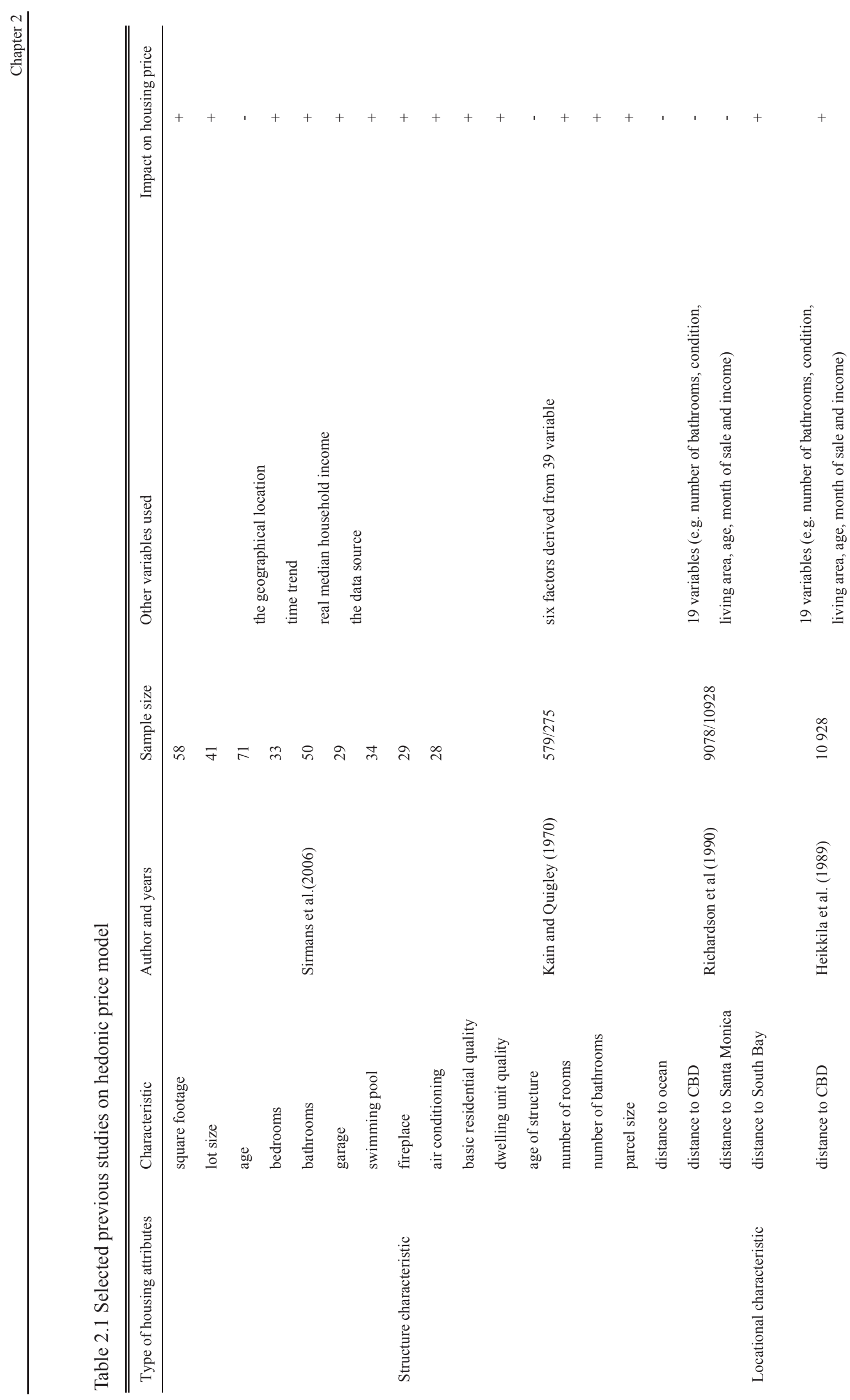




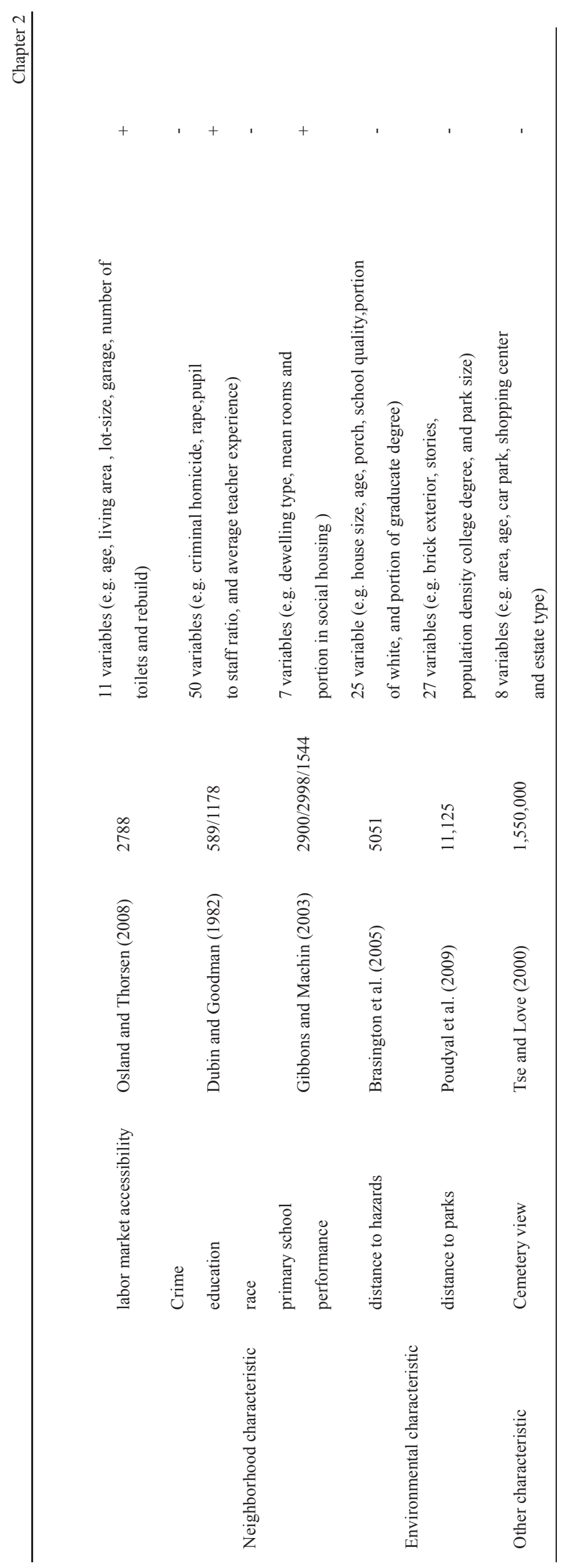




\subsubsection{Structure characteristics}

Structural attributes describe the physical structure of property goods and land parcel. Compared with locational attributes, the structure attributes are easier to account for and accurately perceived. Follain and Jimenez (1985) summarie the most common structure attributes from previous research and note that measures of living space have been reduced to lot size, floor area and the number of rooms whilst structural quality is measured by age, style and interior and exterior quality scores.

Sirmans et al. (2005) summarizes the top twenty characteristics that have been used to specify hedonic pricing equations. He described the total number of times a characteristic has been used and the number of times its estimated coefficient has been positive, negative, or not significant. Age shows up most frequently in hedonic models and typically has the expected negative sign although it is seen to be positive or not significant in some studies. The age effect will depend on the period studied and the age of the city. In historic cities, age may have a positive influence on price, but only in particular housing markets. Age in a modern part of a historic city may have a negative influence since the quality of modern era houses is typically inferior to those in historic quarters. Square footage is the next most used characteristics and typically has the expected positive effect in selling price. Other characteristics that appear frequently are garage, fireplace and lot size. Each typically has the expected positive effect. Garage never has a negative sign but it has been insignificant in a number of studies. Fireplace shows up negative in only a few studies and lot size never shows up negative. Other characteristics that show up frequently are number of bedrooms, bathrooms, swimming pool and basement. Bedrooms show up negative in some studies but bathrooms almost never do. Research shows that a swimming pool never has a negative impact on selling price although it has been insignificant in certain studies, possibly because of the liability of maintenance. Basement is usually positive but sometimes negative even insignificant in some studies, possibly related to 
endemic dampness problems in some property markets.

In addition, Morris et al. (1972), in their pilot study in San Juan, Puerto Rico, examined structural quality by using the dimension of availability of plumbing facilities and other service facilities such as cooking equipment, refrigeration, and lighting. They differentiated plumbing facilities into "inside, for exclusive use," "inside, shared," or "other." These measures, which reflected the quality of the dwelling without associating them with the locational or neighbourhood attributes, were found to be effective proxies for measuring quality features. Kain and Quigley (1970) investigated the impact of housing quality on housing prices. They used measures such as condition of, exterior structure, walls, condition of floors, drives and walks, windows, , and levels of housekeeping. These quality features (e.g., number of bathrooms, the number of rooms and lot size) were found to have as much effect on the price of housing.

Sirmans et al (2006) examined the effect of nine housing characteristics on housing price that appeared in most hedonic pricing models, including square footage, bedrooms, lot size, age, bathrooms, fireplace, swimming pool, garage, and air condition. They found that the coefficient of the square footage, lot size, age, bathroom, swimming pool and air condition are sensitive to some geographical locations and the number of variables in the hedonic model but not to time, household income, or source of data. However, garage, fireplace and bedrooms coefficients are not affected by some geographical location, time, income or the type of data. In contrast, Kohlhase (1991) found that the significance of structural attributes can change over time, and may vary between nations. While attributes relating to the number of rooms and floor area are relatively important across nations, other attributes change with the tradition of building style or the climate.

Theoretically, a property's structural attributes and its location within the city are 
related, since they reflect the growth of the urban structure (Muth 1969), this implies that an element of location will be inherent within the physical structure of the property. This is indicated in studies such as Cubbin (1970) and Kain and Quigley (1970), which revealed a high degree of multicollinearity between structure attributes and the results suffered from spatial autocorrelation.

\subsubsection{Locational characteristics}

A property represents not only a amount of structural characteristics but also set of location specific characteristics, which has long been regarded as the fundamental influence in the modeling of residential location. Von Thunen's classical land use model was the first to formally correlate value with systematic locational characteristics - distance to a central marketplace.

The tradition of neo-classical micro-economic theory(Alonso 1964b; Muth 1969; Mills 1972),developed Von Thunen's model (and the underlying Ricardian value model) to emphasisean 'access-space' trade-off model that describes transportation costs as a trade off against land rents. The trade-off model was developed under the assumption of a monocentric city on an isotropic plane with a housing market in perfect competition. The key idea behind the monocentric model is several restrictive assumptions such as that workplaces are spatially centralized is that accessibility to the $\mathrm{CBD}$ is the major determinant of location-specific land values and site rents. Beckmann (1973) developed models of urban housing markets based on the central assumption that housing and employment accessibility were jointly purchased in the residential choice decision. Most early economic studies of housing price found there is a downward sloping housing price curve with distance from the central business district (CBD). However, the monocentric model has inherent limitations and has increasingly been criticized by researchers (e.g. Boarnet, 1994). The criticisms can be summarized into three types. Firstly, many authors have noted that employment is not 
concentrated in a central business district (e.g. McDonald, 1987; Heikkila et al., 1989). Secondly, it has been questioned whether urban commuters engage in 'wasteful commuting' in their journey to work, which could also be interpreted as the question of whether the distribution of jobs and residence is the primary determinant of the journey to work (Hamilton and Röell 1982; Hamilton 1989). For example, if persons do not choose their residential location to minimize their commute to work, then there are non-transportation factors, which are also influential in residential location (White 1988b; Small and Song 1992). Thirdly, some researchers have questioned the monocentric assumption of exogenous employment location(Steinnes 1977, 1982). The monocentric idea that the residential location is endogenous to employment location, but the employment location is largely exogenous to residential location is questionable. If employment location is endogenous to residential location, the partial equilibrium approach of most monocentric models is inappropriate.

Many scholars conclude that workplace accessibility has been over emphasized in the urban economics empirical literature. For example, as stated by Heikkila et al. (1989):

"with multiple-worker households, multiple workplaces are common; given a high degree of residential mobility, sites offering accessibility to many employment nodes are more valuable because it is not very likely that successive owners will work in the same workplace".

Richardson et al (1990) found a significantly negative value of the coefficient related to distance from the LA CBD in 1970; and this variable was found not to influence house prices in 1980. McMillen (2003) found that in many cities the CBD no longer appears to exert a significant influence on house value. Take Chicago as an example, long viewed as a monocentric city but one in which the centrality has declined steadily in importance over time.

In fact, cities rarely have a simple monocentric structure, and the monocentric city is a 
special case of the standard urban model (Bender and Hwang 1985). Employment and amenity centers are often located outside of the city centre, which may cause the house price gradient to be complex (Orford 1999). Button and Taylor(2000)noted in the 1990s, when metropolitan areas were in a state of reformulation that no longer is the CBD the only place one may find gainful employment, as many suburban employment center have arisen to combat its draw. On the other hand, there is little consensus as to the appropriate method for identifying the sub-employment centre. For example, Giuliano and Small's clustering methods (1991), and McMillen's nonparametric methods (2001). Clustering methods rely on ad hoc definitions of density and total employment cutoffs and parametric models make strong assumptions regarding parametric form, leading to misspecification (Redfearn 2007). Empirical research on the nature of property price with a polycentric urban context has been scarce. For example, in the case of Baltimore, Dubin and Sung (1987) conclude that 'the CBD appears to behave like the other secondary centre: it has an impact, but this effect is limited to a relatively small area'. Similar results were also found by Jackson (1979) in Milwauke and Bender and Hwang (1985) in Chicago.

\subsubsection{Accessibility}

Accessibility has been discussed in geographic contexts from numbers of perspective (Kwan 1998). There is little consensus on the precise definition of accessibility. Stewart described accessibility as the population-over-distance relationship or 'population potential', while Hansen (1959) defined accessibility as: 'the potential of opportunities for interaction'. Accessibility is a measurement of 'the spatial distribution of activities about a point, adjusted for the ability and the desire of people or firms to overcome spatial separation' (Hansen, 1959). Accessibility also can be defined as 'the ability of individuals to travel and to participate in activities at different locations in an environment' (Des Rosiers et al. 1999). According to Des Rosiers et al. (2000), accessibility relates to the ability of the individual to travel and 
to participate in activities at different locations. In a transportation model, accessibility is defined as '......the distribution of some defined activity measure versus the travel impedance (time, cost and distance) to reach that activity from the selected zones ......'(Adair et al. 2000).

Ball (1973) found that while most studies showed distance variables to be significant, not all agree on the measure of distance. Recently, more sophisticated measures of accessibility have been proved toper form better than purely Euclidean distance in many studies on property value (Niedercorn and Ammari 1987; Hoch and Waddell 1993). Heikkila et al. (1989) suggest considering the possibility that accessibility to nodes other than the CBD might be important. Bowes and Ihlanfeldt (2001) proposed that railway stations raise the value of nearby properties, as that reduce people's commuting costs, and station area should therefore be better able to attract retail activity. Various researchers have explored the relationship between specific measures of accessibility and property value, and as showed in figure (2.3), accessibility has been measured at aggregate level and individual level. At individual level, accessibility could account for the distance or time cost from a location to certain facilities, such as school, transit station, employment centre, shopping centre and so on (e.g., Landau et al., 1981, Henneberry 1998). 


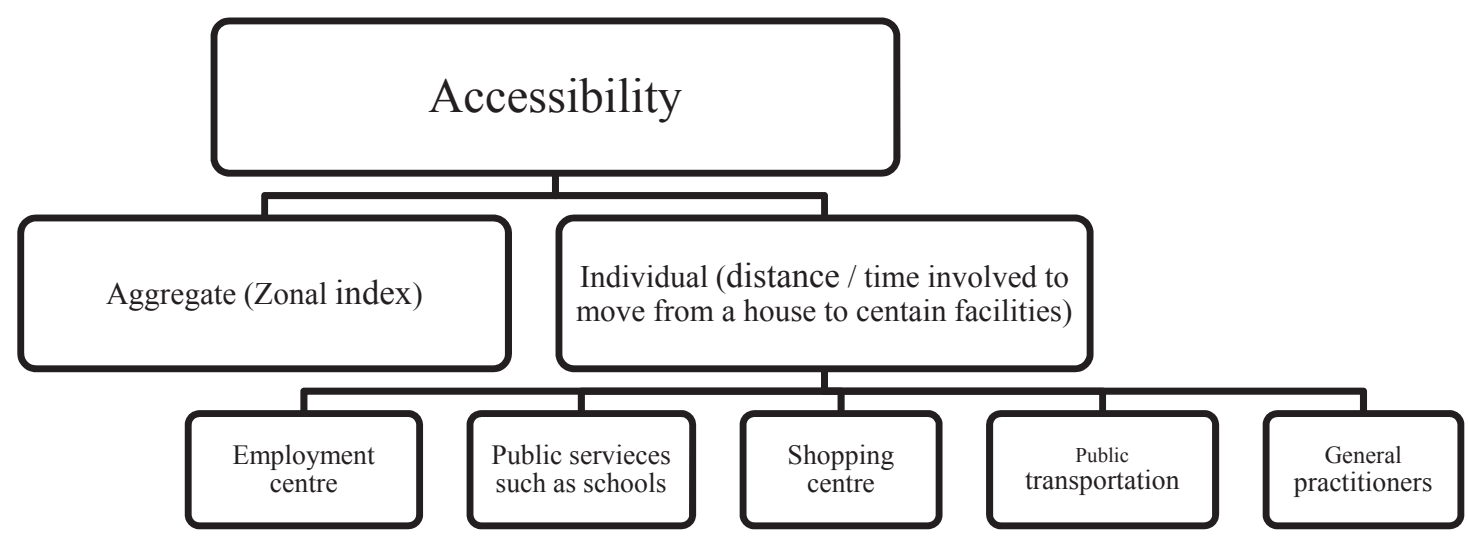

Figure 2.3 Accessibility measurement types

Source: Ismail (2005)

Debrezion et al. (2006)explored the impact of the railway network on house price in the Netherlands by hedonic price approach. The railway access variables the authors used include the distance to railway station, the frequency of train services and to distance to railway tracks. They found that housing in close proximity to a railway station commands a market price that is about $25 \%$ more expensive than equivalent housing at a distance of $15 \mathrm{~km}$ or more from a station. Munoz-Raskin (2010)paid attention on the relationship of bus rapid transit and property values within walking area. He found housing market places value premiums on properties in immediate walking proximity of BRT feeder lines.

In contrast, Andersson et al. (2010) examined the effect of High-speed rail station (a long-distance rail) accessibility on real estate price in Taiwan. The estimated results show that HSR accessibility has at most minor effects on house price. Rolon (2005) also found a new station does not bring substantial accessibility improvement and the marginal effect on land and property values is negligible. However, there was a negative impact of proximity to a transit station due to the noise, vibration, pollution, visual impacts and safety issues (Bowes and Ihlanfeldt 2001). 
However, at the aggregate level, accessibility can be considered as a point of attractiveness or proximity to an opportunity. For example, Hwang and Thill (2010) examined the impact of job accessibility on housing price in the Buffalo and Seattle metropolitan areas. They compute a travel-time based job-accessibility measure at the employment level of census tracts, according to Hansen (1959)'s formulation. The results suggest that suburbanites are more willing to pay for additional increase in job accessibility in housing consumption than urban residents in the Buffalo-Niagara Falls MSA, whereas the situation is opposite in Seattle.

Song and Sohn (2007) also criticized accessibility measures based on distance from a housing unit to the $\mathrm{CBD}$, regional and commercial center, arguing that it cannot capture the overall level of accessibility for retail service. They applied a spatial accessibility index to evaluate the effect of enhanced access to retailing in the single family housing market in the city of Hillsboro, Oregon. This spatial accessibility index considered the numbers of neighborhood retail store, the size of retail store, as well as the distance to the retail store by units of census block (e.g. Weibull, 1976, Shen 1998). The results showed that spatial accessibility to retailing as a service is capitalized into residential price.

Adair et al. (2000) focused on the relationship between accessibility and housing price in the Belfast urban area. Instead of traditional studies using the CBD as a reference for accessibility indicator, they calculated the accessibility index by considering all trip attractors and generators in the area of 182 traffic zones with sample size of 2648. For whole housing market, they found accessibility is of little significance in explaining variation of house prices. However, the authors found that accessibility can be an important influence at sub-market level, particular in low-income areas. Johnson and Ragas (1987) found that the biggest obstacle to finding declining rent gradients within a small area is that there are many other confounding factor that 
affect the land rent other than just spatial locational characteristics.

Another issue emphasized by Song and Sohn (2007) is the inaccuracy of the accessibility measure due to spatial aggregation. Some spatial information is lost and become insignificant as 'households in each zone or area are typically represented by a single point (i.e. centroid or weighted center) in calculating distance to and from the zone'. Furthermore, they argued that the arbitrariness of spatial units could distort the real accessibility level of individual household. Cheshire and Sheppard (1997) also argued that much of the data used in hedonic analyses still lacks land and location information. Different accessibility indices can interpret the different locational information but not all the indices have proved to affect housing value. Besides that, these aggregate empirical studies generally have not found accessibility to be as major a factor in controlling residential mobility as traditional trade-off models imply (Adair et al. 2000).

\subsubsection{Neighborhood}

Neighborhood attributes are also typically included in the estimation of housing price models. Important among these are income level (which is a surrogate for among other things, neighbor externalities, the 'snob' factor, quality of housing, level of expenditure on housing investment and maintenance, school quality and so on). Generally, higher income neighborhoods are assumed to be of higher quality (e.g. higher quality education and lower crime rate). This leads to the idea that all households prefer to live in higher income neighborhoods. Set against this is the observation and theoretically plausible idea that households prefer to live in neighborhoods dominated by households similar to themselves (Gans 1963). Hedonic models of housing values that use a set of social and economic status variables (in terms of age, income, ethnicity and lifestyle), controlling for neighborhood and amenity quality (e.g. school) can help tease out these effects. 


\subsubsection{School quality}

There is a widely recognition that school quality is the most essential determinant of housing price, in particular within the US. and UK contexts, as it is close related with local property tax bands. Generally, in the field of education, some author choose a indicator to control for school quality, such as pupil-teacher ratio and standardized test scores (e.g. Oates, 1969, Haurin and Brasington, 1996).

Dubin and Goodman (1982) estimated the impact of school characteristics and crime measure on 1765 house prices in Baltimore in 1978. They measures of school characteristics by the pupil-to-staff ratio, average teacher experience, the percent of staff with masters degrees or above, and a battery of third and fifth grade test scores. As the school variables were highly correlated, they use principle component analysis to reduce the data. They confirmed the school characteristics had a significant effect on house price, but it is still difficult to determine which school characteristics contribute more effect.

Hefner (1998) examine the impact of school characteristics on house price by conducting two measurements for school quality. The first measurement focus on management, including teacher's salary, teacher / pupil ratios, teacher tenure, and percentage of teachers with advanced education degrees. The second measurement is considered by support for and participation in gifted and talented programs. He found that administrative and leadership choices made by school and parents can increase the prices of surrounding properties.

Gibbons and Machin (2003) investigated the impact of primary school performance on housing prices in England with a pooling data set of 7444 "postcode area" in the years 1996-1999. They serve school type as the instrument for school quality, and 
find a positive effect of local school quality on house prices. The results show that one percentage increase in the proportion of children meeting raises property values by $0.67 \%$.

However, the question of how change in school characteristics relate to changes in real estate value remains open in part because, as Mieszkowski and Zodrow(1989) note, many existing efforts to determine the extent of capitalization have been flawed due to inadequate data.

\subsubsection{Social economics status and ethnicity}

The socio-economic characteristics of the neighborhood, such as the social status and population characteristics (in terms of age, income, ethnicity and lifestyle) of a neighborhood, also play a role in the choice behavior of house buyer, and therefore have an effect on house price (Visser et al. 2008). Dubin and Sung (1990) showed that the social-economic status and racial composition of the neighborhood affect housing price more than the quality of public services. Racial segregation behavior studies in some US cities (Harris 1999)may influence housing price, depending on a community's willingness to pay to keep its identity

Baumont and Legros(2009) examined the impact of neighborhood on the housing value in the Metropolitan Area of Paris. Social capital, social status, social externalities and urban renewal policies have positive or negative impacts on housing prices.

Schafer (1979) looks at Boston 1970 census data. When the data are divided into submarkets defined as the central city ghetto, the central city transition area, the central city white area, and suburban white area, he reports that price differentials paid by blacks vary greatly, depending on the submarket. He find that house prices are 
higher in the ghetto and transition area, relative to the white area.

Dougherty et al. (2009) measure the effect of both elementary school test scores and racial composition on household' purchase choice over a 10-year period. Overall, while both test scores and race help explaining the variation in housing price significantly, and they found that the influence of school performance declined, but racial composition became nearly seven times more influential during the study period.

Differences in house prices across racial groups have been carefully analyzed in the past. As Yinger (1979) and Chambers (1992)point out, it is crucial to include indicators for the household, the neighborhood, and the submarket, while controlling for the characteristics of the house when testing for price differentials. If relevant characteristics are excluded, the estimated coefficients will be biased.

\subsubsection{Environmental}

Since Ridker and Henning's (1967) pioneering study, there has been growing interest in using property value as a source of information on the benefits to be expected from controlling environment disamenities. Or more generally, property price models have become one of the common ways of valuing environmental externalities. Most commonly the method is applied to variations in housing prices which reflect the value of local environmental indicators, and variety of empirical studies have used a single environment indicator in a hedonic price model(Anderson and Crocker 1971; Wilman 1981; Murdoch and Thayer 1988). Generally, environmental characteristics can be subdivided into two categories environmental quality and environmental amenities. Environmental quality includes air pollution, water pollution, or noise, while environmental amenities can be interpreted as aesthetic views and proximity to recreational sites. 


\subsubsection{Environmental quality}

Some studies have examined the impact of environmental quality of air, water, or traffic on house price using hedonic models. Generally, these studies show that urban disamenities have a negative effect on house price, which means people have a low demand and do not have the willingness to pay for more on these characteristics - on the contrary, they are willing to pay more for less of them. Day et al. (2007) and Bateman et al. (2001) consider aircraft noise, while Schipper et al. (1998) and Nelson (1982) assess the impact of multiple resources of noise from transport. Air quality has been evidenced to have a negative relationship with property value (Graves et al. 1988; Smith and Huang 1995). Water quality, such as $\mathrm{pH}$ level, clarity or visibility has been found to be positively and significantly related to sale price (Steinnes 1992; Michael et al. 1996). Proximity to hazardous waste sites unsurprisingly have a negative impact on property value (Kiel 1995; Farber 1998).

Brasington et al. (2005) use the pollution site data of Ohio in US to examine the relationship between house price and environmental disamenties. They used spatial autoregressive method to confirm that nearby point-source pollutants depress house price. Epp and Al-Ani (1979) studied waterfront residential properties located along small rivers and streams in Pennsylvania and found that $\mathrm{pH}$ levels low enough to limit recreational use affect housing price. They found that acidity from minerals and carbon dioxide, which affects $\mathrm{pH}$ levels, significantly influenced housing price.

A distinction can be made between studies principally aimed at deconstructing house price and those undertaken to value an environmental 'bad'. It is likely that the latter kind of impact studies may not be so comprehensive in identifying a full range of independently predictive variables. Such evaluations have been criticized for difficulties in capturing imperfect knowledge on the attributes of each location and 
measuring intangible influences and individuals' perceptions (Wardman and Bristow 2004).

\subsubsection{Environmental amenities}

As mentioned above, proximity to a certain environmental amenity, such as a river, and open space, could be considered as a dimension of accessibility adding value to property values. Stegman (1969), Richardson (1977) and Pollakowski (1982) show that house prices are also determined by the environmental attributes of the location, which connote lower accessibility of areas peripheral to the city centre tends to outweigh increased access to open space. Gillard (1981) argues: "even when a park may not be used for recreation because of crime problems, it may still be valued for aesthetic reasons by residents with a view of the park". McLeod (1984) discovered that the river views were particularly important, and had a greater influence than a view of park. In particular, industrial, business and transportation land uses can have a negative effect upon property with respect to aesthetic qualities (Powe et al. 1995).

Public open space and urban parks could enhance the economy in environment, as well as quality of life, by improving air quality, providing recreational opportunities, and enhancing aesthetic value, among many other benefits (Nowak and McPherson 1993). Previous research have revealed that the price of house increases with proximity to nearby parks (Tyrväinen 1997; Thorsnes 2002), while, other studies reveal that increasing the size of urban parks increase the housing values nearby (Tyrväinen 1997). This lends weight to the idea that it is size of parks rather than number per se, that matter in meeting the open space needs of a city.

Poudyal et al. (2009)examine how the demand for green parks captured by property value. They employed a traditional hedonic price model to confirm that urban recreation parks increase nearby property values. Increasing the average size of parks 
by $20 \%$ from the current level increased the per household consumer surplus by 160 dollars.

Netusil (2005), investigated how far open space can affect house price, taking the empirical study of Portland, Oregon. At the radii of 30 meters to the open space, the study found that impact of locational advantage on $\mathrm{n}$ the sale price of homes is insignificant. However, at distances greater than 30 meters and up to 450 meters from open space, homes were found to sell for statistically greater price than homes located over 450 meters from open space.

Lutzenhiser and Netusil (2001) used the same data, to explore the open space effect more deeply. They classified open space into urban park, natural park and specialty park, and found of great significance were natural area parks-homes within 450 meters. Other types of open space having a statistically significant influence include golf courses (13.3\%), specialty parks/ facilities $(8.5 \%)$ and urban parks $(1.8 \%)$. They find being proximity to open space does have positive impact on property values, but this is still dependent on the type of open space and distance from the open space.

Anderson and West (2006) explored the effect of neighborhood parks, regional, state, federal parks,natural areas and cemeteries on property value in the Minneapolis-St. Paul metropolitan area. They find that the value of properties proximity to neighborhood parks and special parks falls respect to the distance to the CBD increases where has high population density and income. The benefits of proximity to neighborhood parks on housing price become higher when neighborhood has more children.

\subsubsection{Others}

In addition, there are some local context attributes, which influence property values, 
for example, Tse and Love (2000) found that a cemetery view has a negative impact on a property's price in Hong Kong. Generally, dwellings that have a cemetery view are not accepted, as that is bad fengshui (geomancy).

Interestingly, there have been some studies that demonstrated the influence of fengshui beliefs in the power of "lucky" and "unlucky" properties. For example, Bourassa and Peng (1999b), who used sales transactions for 1989 to 1996, found that lucky house numbers (e.g. 3, 6, 8, and 9) have significant positive hedonic prices and are capitalized into the sale prices of houses in Auckland, New Zealand. Chau et al.(2001) also found similar results in the predominantly Cantonese society of Hong Kong. Their results, however, showed that lucky floor numbers (e.g. 8, 18, or 28) are sold at significantly higher premiums during periods of property boom than during property slumps.

\subsection{Summary}

This chapter presents a wide-ranging literature review of hedonic price models, which can be summarized from a numbers of aspects.

Firstly, based on Rosen's work (1974), it is possible to state that under a perfectly competitive market, and when demand equals supply, the implicit price of each attribute in the hedonic price model is the price people are willing to pay for each characteristic. However, there are many scholars who have criticized Rosen's hedonic price estimation for the assumption of market equilibrium, in which case the implicit price of an attribute estimated is not strictly equal to the marginal willingness to pay.

Secondly, it has been noted that economic theory gives no clear guidelines on how to select hedonic price functional form. Since, the study area of the first two empirical studies of this thesis is a part of metropolitan area of Cardiff, UK, and Orford (1999) 
explored the impact of locational externalities on housing price in Cardiff, choosing the semi-log function form, this study will follow Orford (1999)'s approach. This is further backed up by the consensus in the literature that semi-log equations have a more meaningful and intuitive interpretation.

In addition, the literature reviewed also indicates that the hedonic price model suffers from a series of potential econometric issues, which could lead to estimation bias, such as spatial autocorrelation, multicollinearity, heteroscedasticity, and non-measurement of housing quality change. Thus, several types of econometrics tests will be applied in the chapters that follow, to make sure the models correctly or as accurately as possible, estimate the implicit prices of housing attributes, including accessibility. Among all these econometric problems, multicollinearity is most fatal for the estimation, as it can result in an coefficients with opposite signs to their real relationship. Therefore, it is necessary to specify the function form, and choose the variables carefully.

Thirdly, despite of the importance of housing submarkets, there is little consensus on how to specify submarkets, or identify their boundaries. Thus, this study attempts to contribute to the theory and practice of identifying housing submarket using hedonic price models. The traditional method mainly emphasizes that within geographic area the price per unit of housing characteristics is constant, which means within a certain space, people have identical choice preferences. In that case, they could use school districts, postcode areas, and so on, and even include building types to specify the housing submarket. However, there arises a question of how to specify housing submarkets where the building type is homogeneous, and the price per unit of housing characteristics is not constant within some geographic unit. Indeed, some scholars have approached this matter using cluster analysis for non-spatial information by minimizing estimated error for social economics indices, These alternative methods are criticized since the results are unstable over time, they require high quality 
database and are difficult for policy-analysts to interpret. The innovative method I explore in this thesis is to see if detailed morphological metrics can be used in defining housing sub-markets. This takes the discussion of accessibility in hedonics a step further, to see if fine-grained systemic accessibility measures, taken from an urban grid, can not only help refine the prediction of individual house prices but also help define geographical areas with stable and homogeneous valuations of housing attributes.

Fourthly, it is found that housing attributes can be divided into structural attributes, locational attributes, neighborhood attributes and environment attributes. Based on the discussions above, it is found that there is a debate regarding the locational attributes that influence house price: empirical evidence can be contradictory. The New Urban Economics theory suggests that in a monocentric model, emphasis on the location (the distance to $\mathrm{CBD}$ ) is most essential for property value, because it accounts for the minimum travel cost, time and distance behavior. The result is a negative gradient curve demonstrating the relationships between distance to a centre and housing price in markets. However, due to urban expansion and polycentricity and the growing complexity of urban systems, many empirical studies have found that the power of location attributes have become weak. Current studies measure accessibility at both an aggregated and disaggregated level. However, aggregated measurements apply advanced algorithms calculating potential opportunities or attractiveness, which have been criticized for the loss of spatial information when aggregated. By contrast, disaggregated measurement requires a prior specification for terminals (such as bus station, train station), and they mainly rely on Euclidean distance measurement.

Hence, the focus of this thesis attempts to contribute the hedonic price theory by following aspects:

Firstly, previous studies need a priori specification within a pre-defined area, 
identifying the impact of local attractions on housing property, and most of them ignore the spatial information contains in street layout, which is most essential element in the field of urban planning. Furthermore, compared with tranditional measurement for locational attributes, the accessibility derived from urban configuration does not require a priori knowledge and can be easily employed at disaggregated level.

Secondly, despite previous specifications for housing submarket in developed countries, there is less knowledge about how to delineate sub markets in property markets where the building type is simplex (apartments) and social neighbourhood characteristics are not long established and changing quickly over time, such as most cities in China. Thus, I attempt to establish a new framework for delineating submarket in that situation, by clustering urban configuration features, as urban configuration features are assumed to be associated with both spatial information and people's preference.

Thirdly, there has been little evidence of the micro-level determinants of house price volatility in urban land use planning. It is known that China is undergoing a process of rapid urbanization, which in terms of scale is perhaps the largest the world has ever experienced. Although some researchers have investigated the impact of accessibility value, they have failed to confirm the dynamic relationship. Thus, I posit that transformations in urban configuration accompany the urbanization process; and that the corresponding continuous changes in street network configuration associated with urban growth and the attendant changes in accessibility are one of the key determinants of micro-level house price volatility in a city. 


\section{Chapter Three:}

\section{Space syntax methodology review}

\subsection{Introduction}

Space syntax is a street network method, which was developed in the 1970s at the University of London as a way to quantify urban morphology features and record movement and interaction within cities and buildings. In the book of The Social Logic of Space (Hillier and Hanson 1984), they firstly argued that spatial layout or structure has a great impact on human social activities. Recently, the approach was refined by Hillier (1996), particularly focus on the arrangement of spaces and possibilities and patterns of movement through the 'spatial configuration'. Over the past two decades, space syntax theory has provided computational support for the development of urban morphological studies, revealing of the characteristics of spaces in terms of movement and potential use. Indeed, space syntax attempt to define the elements of urban form by geometric accessibility, measuring the relationships between street segments by a series of measurements, such as connectivity, control, closeness and betweeness (Jiang and Claramunt 2002).

Indeed, as a technique, space syntax is powerful and successful, as it can describe a complex urban configuration system, providing direct interpretation to help architects, urban planners and sociologist to understand the impact of spatial configuration on social activities both theoretical and practical. For example, for the regeneration project at Trafalgar square, London, the Space Syntax research method provided an initial insight of tourists' activity patterns in the Trafalgar Square.

Despite its success, there have been critics of the space syntax research method, 
mainly focusing on definition of axial lines and the unique set of axial lines. For example, Ratti (2004) criticized that the appropriateness of 'axial maps' for describing the urban spatial structure. It has also been suggested that the definition and theoretical meaning of the axial map is not well-enough linked to other graph-theoretic representation of street network (Steadman 2004).

The motivation of this chapter is to provide deeper insight into the space syntax method, discussing several issues. Firstly, what is different about space syntax network analysis compared with traditional geographic network analysis assessing accessibility? Secondly, the algorithm of the space syntax method and three type of accessibility measurement of space syntax are discussed. Thirdly, some key criticism of space syntax will be summarised. Fourthly the social economic fields that the space syntax method has been applied to will be discussed.

\subsection{Overview of urban morphology analysis}

The study of urban morphology, frequently referred to urban form, urban landscape and townscape, has an extensive literature in three genres. Firstly, in field of human geography, especially in Britain, there is an "indigenous British geographical tradition", which heavily influenced by M.R.G. Conzen. Conzen develops two of his key ideas, the burgage cycle, and fringe belts. The Conzenianis more interesting in the description, classification and exemplified the characteristics of present townscapes based on the survey. Recently, the typical works contributed to of this tradition are from Whitehand (1967; 1972, 1987a, b; 1992)and Slater (1988)..Additionally, by contrast, European traditions (e.g. Caniggian School) takes the views from architect, emphasizing the components of the urban structure, including its elements, structures of elements, organism of structures(Sima and Zhang 2009). In architecture and planning theory. Many scholars concerned urban morphology with prescriptive and utopian for 'ideal cities'. For example, The Garden Cities of Howard (1965), Le 
Corbusier's Ville Radieuse (Corbusier and Guiton 1981),and Wright's Broadacre (Wright 1935)are the outstanding examples of this genre.

However, the most prominent exponents of the critique from Lynch (1960), Jacobs (1961) and Alexander (1964, 1974), requires to think city planning from anthropology perspectives, which should be based on observation of what actually works in real cities. This sowed the seeds of a mathematical approache for quantifying urban morphology.

Alexander's work first attempt to introduce formal mathematical concepts into the debate. Some scholars interested in bringing mathematical tools into the realm of urban morphology, in particular graph theory and set theory, and attempted to link this idea with what works in the urban design arena (see. March and Steadman 1971, Martin and March 1972 ,Steadman 1983 ). For example. Q-analysis (Atkin 1978) was also influential. Work focusing on possible graph representations of urban form (Krüger 1979) exemplifies the approach. Under this background, the method of space syntax was gestated by Hillier and Hanson (1984b), which is based on a graph representation describing cities as systems of open space.

\subsection{Accessibility types}

"Accessibility is a concept that has become central to physical planning during the last fifty years.” (Batty 2009).

In the field of population geography, Stewart (1947) firstly used the concept of graph theory defining the gravitational potential by the weighted sum of forces. Hansen (1959) and Wilson (1970) started with a graph theory view to explore the spatial system, identifying accessibility as a key element in spatial interaction, while Haggett and Chorley (1969) established the central analysis approach to analyse spatial 
relationships.

Batty (2009) summarized three types of accessibility. Firstly, type one accessibility defines how proximate or 'near' an individual is to 'opportunities', measuring the size of the opportunity at some other place or location and inversely moderating the cost of accessing the opportunities by the distance or time. The other two types of accessibility are based on a network. Type two accessibility more focus on the Euclidean distance between one location and another, measuring this as the sum of the shortest routes in a planar graph.This measurement is popular in traffic models, and high accessibility normally is associated with minimum distance, travel time and travel cost. In the metric geographical network analysis, nodes or vertices are defined as the intersection or junction of streets, while the links or edges are street segments linking two intersections. This is called the prime approach. In the last twenty years, a third type of accessibility measurement has emerged which seems more abstract. The type three accessibility is based on the dual approach, which defined on the links of the original graph, rather than the nodes, applying degree $\left(c^{D}\right)\left(\right.$ control), closeness $\left(c^{C}\right)$ ("integration"in space syntax), and betweenness $\left(c^{\mathrm{B}}\right)$ ("Choice" in space syntax) as measurement of centralities (Bavelas 1948, 1950; Leavitt 1951; Shimbel 1953; Shaw 1954, 1964).

The accessibility measured by Space syntax method(Hillier and Hanson 1984b)is belongs to the third type, which is also called dual approach. It is more abstract, focusing on links (street) connection in a planar graph. Similar dual approach methods have also explored gradual directional change (Dalton et al. 2003), characteristic point (Jiang and Claramunt 2002), street-name change (Jiang and Claramunt 2004). 
a. The Planar Graph as Primal

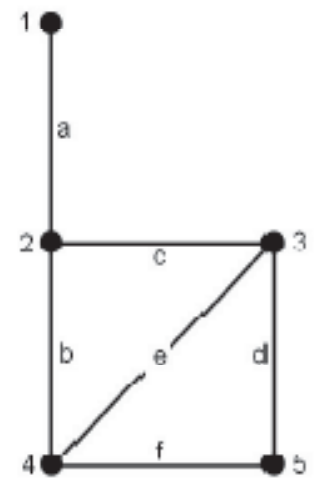

b. The Dual of the Planar

Graph

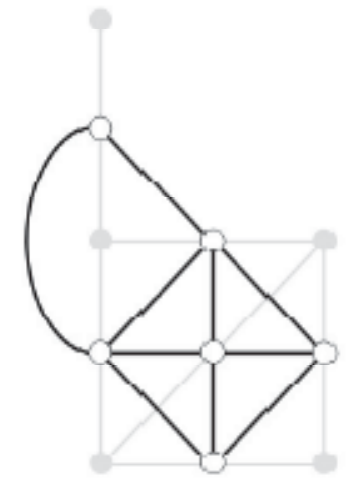

Figure 3.1Conventional graph-theoretic representation of the street network

Source: (Batty 2004a)

Many researchers have discussed the substantial differences between the prime and dual approach, showed in figure (3.1), and arguing which approach is more effective. Batty (2004a) states the dual approach breaks the clear links between the Euclidean and topological space and also making the visual analysis more difficult, compared to the prime approach, he agrees that both methods are powerful to explain the same problem. Porta et al.(2006) introduces a new geographical network analysis methodology, known as multiple centrality assessment, based on the prime approach. Comparing results for the two methods, they point out that the prime approach is not efficient in measuring the $c^{\mathrm{C}}$ closeness and is much more fragmented than the dual method, which is more generalized. However, he emphasizes that the prime approach is more compatible with other fields, and is more subjective, intuitive and practical. Hillier and Penn (2004) emphasises that the dual approach use of topological distance prevents border effects.

\subsection{Space syntax algorithm}

What is original to space syntax is the important insight that the pattern of movement 
in a city or urban area is likely to be shaped to an extent by the topology of its route network alone. The basic analytical procedure of the original space syntax method is divided into two steps. The first step is to create an axial map, of a certain area at any given scale. At the early stage, the axial map is based on experiences, which starts with identifying the fattest convex spaces. A 'Convex space' showed in figure (3.2), can be defined as polygons where no lines can be drawn between any two points $(\mathrm{x}, \mathrm{y})$ in the space which go outside the area. The longest line indicate the most optimal convex space with the maximum perimeter ratio. After identifying the fattest convex space, it is possible to draw the longest axial lines within the convex space and then draw the second longest axial and continue until all the convex spaces are covered. The whole process should result in the fewest axial lines representing a one-dimensional system of the spatial layout (Hillier and Hanson 1984b). Space syntax makes a default assumption that people will move where they can see, and 'Convex space' could indicate people's movement in a visual area. Urban space could be decomposed into numbers of the 'Convex space' by people's visual zone contained in urban spatial structure or morphology, thus, the urban space could be transformed into line-line graph with 'longest and fewest' axial lines. 

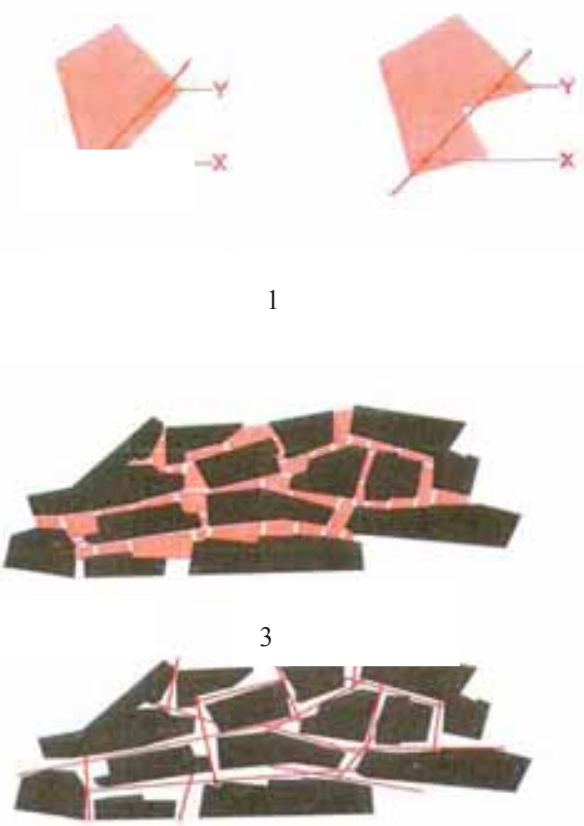
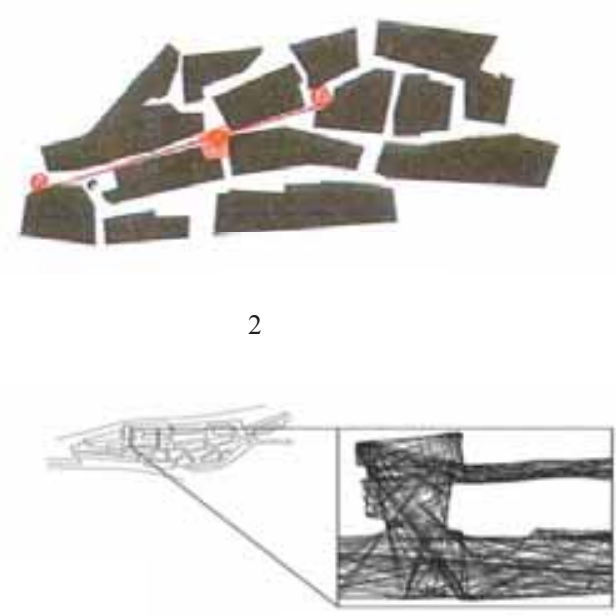

4

Figure 3.2The process of converting the "Convex Space " to axial line map

Source: (Hillier and Hanson 1984b)

The results in the complex urban configuration system being represented as a one dimension graph $\mathrm{G}(\mathrm{N}, \mathrm{K})$, a mathematical entity defined by two sets, $\mathrm{N}$ and $\mathrm{K}$. The first set, $\mathrm{N}$, denotes a nonempty set of $\mathrm{N}$ elements called nodes, vertices, or points, and $\mathrm{K}$ is a set of $\mathrm{K}$ elements containing unordered pairs of different nodes called links or edges. Once the representation of morphology has been created, measures of spatial characteristics can be analyzed. Each axial line represents a node $\mathrm{N}$ in the graph and each intersection between lines represents a link K. An indicator for measuring non-metric topology is depth, which is defined by the number of turns from one axial line to another line, or the links from one node to another node. Depth can be used to measure patterns of line connectivity called integration(Steadman 1983; Hillier and Hanson 1984b). This line graph approach has proven to be unexpectedly successful in predicting urban movement and measuring urban structure change(Hillier et al. 1993a; Hillier 1996). 

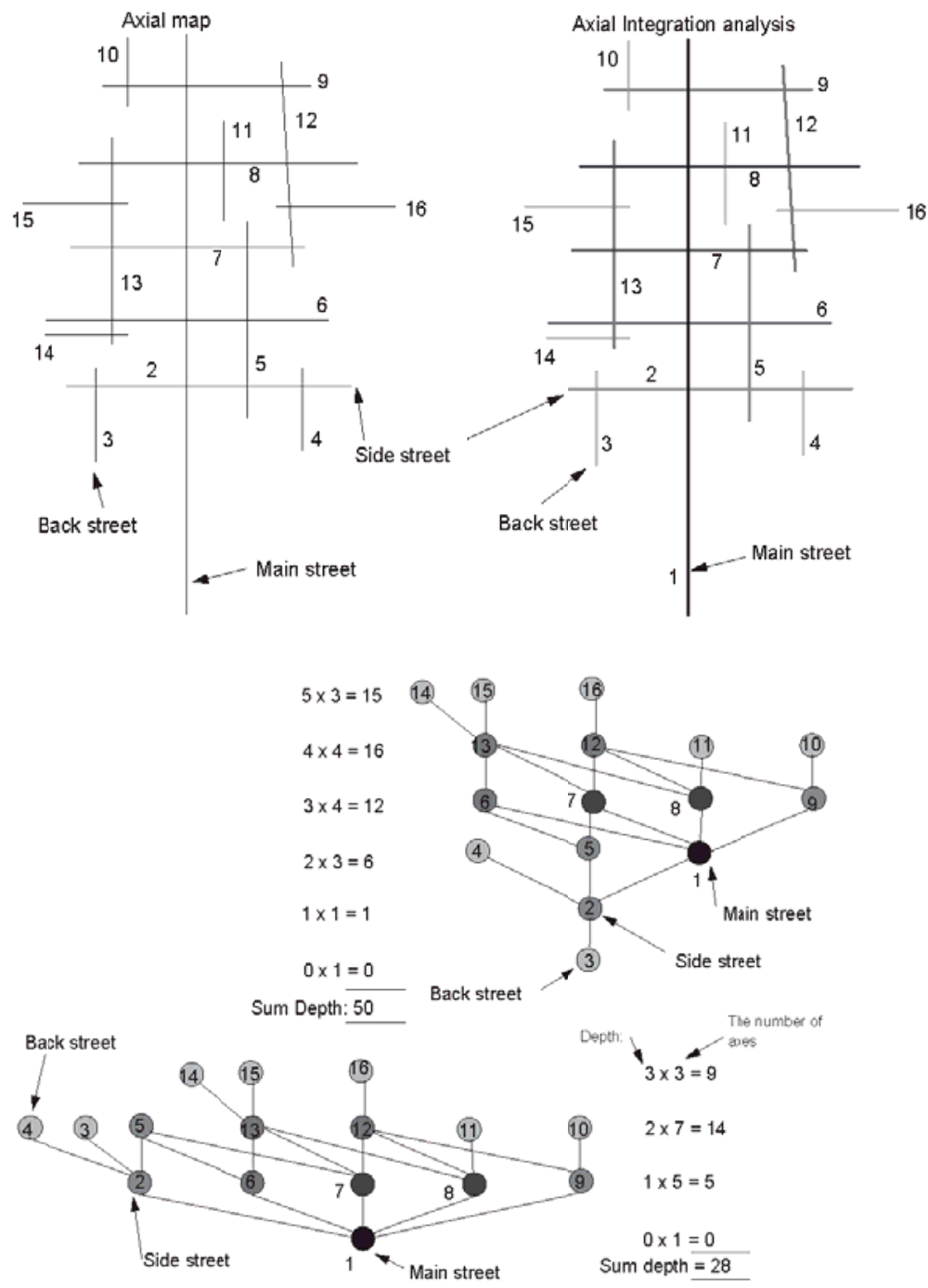

Figure 3.3 calculation of depth value of each street

Source: author

Figure (3.3) shows how space syntax integration value is calculated for each axial line based by accounting the numbers of depth. According to Hillier and Hanson's work (1984),The formula for integration of space syntax axial lines, has several steps as follows:

1.Sum Depth $=\sum_{j=1, j \neq i}^{K-1} d_{i j}$ Equation (3.1) 
Where:

$\mathrm{K}$ is the total number of nodes (axial line) in a graph

$d_{i j}$ is the numbers of depth from node $i$ to node $j$.

2. Mean depth $(\mathrm{MD})=$ Sum Depth $(\mathrm{SD}) /(\mathrm{K}-1)$.

Equation (3.2)

3.Relative Asymmetry $(\mathrm{RA})=2(\mathrm{MD}-1) /(\mathrm{K}-2)$

Equation (3.3)

4.Real Relative Asymmetry (RRA) $=$ RA/ Dk

Equation (3.4)

Where:

$D_{k}=\mathrm{Nlog}_{2}\left(\frac{\mathrm{N}+2}{3}\right)-(\mathrm{N}-1)$ denotes the total depth of the root in a diamond-shaped graph

5. Integration $=1 /$ RRA

Equation (3.5)

Relations of depth are derived from the notion of asymmetry, as spaces can only be deeper than other spaces when passing through intervening spaces to arrive to them. Mean Depth provides the basis for RA, which provides a normalization of the mean depth measure between the deepest a node could possibly be (at the end of a sequence) and the shallowest it could be (when all other nodes are directly connected to it). This will give a value between 0 and 1, with low values indicating a space that tends to integrate the system, and high values a space, which tends to be segregated from the system.

RRA provides a relativisation to allow for comparisons of depth between different sized spatial system. RRA eliminates the effect caused when real spatial systems get larger (are composed of more nodes). With a larger system (area) a node becomes relatively shallower when considered relative to how deep they could possibly be 
given that number of nodes. Normally, it allows us to compare the integration value for different urban system, such as London and Beijing; which requires eliminating the effects of the size on the RA of any space.

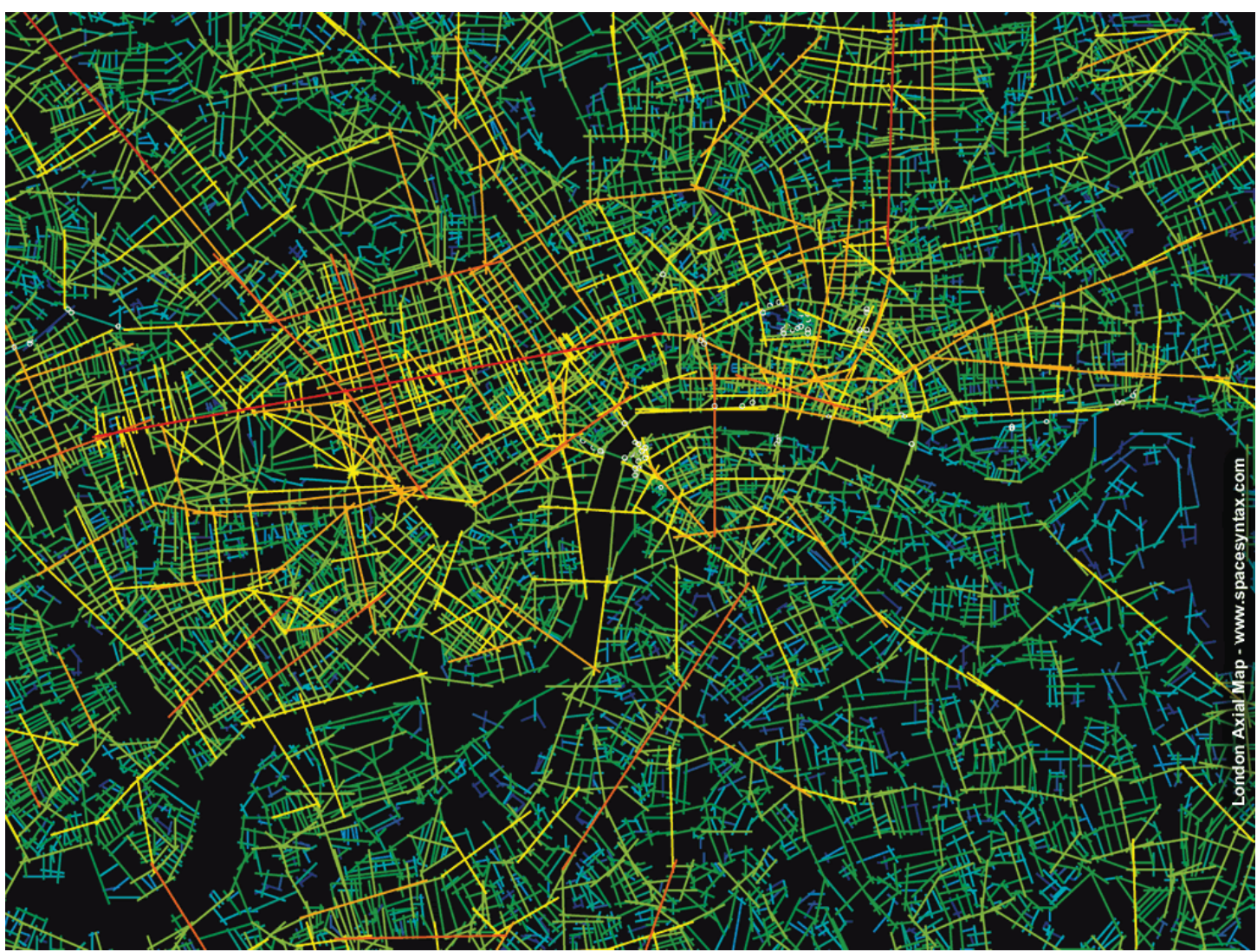

Figure 3.4Integration map of London

Source: www.spacesyntax.com

Researches using the space syntax methodology, utilize the so called global integration $(\mathrm{RN})$ method to measure the relative accessibility of a space within a spatial system, measuring how many locations in the city are relatively strongly integrated (connected) and how many are less integrated and probably belonging to the city fringe. Higher integration values are assumed to correlate with higher rates of movement and activity. Spaces with few connections that lie "deep" within a system have lower integration values and often experience lower levels of activity. In the space syntax graphic, red lines represent the highest integration value; dark blue lines represent the lowest integration value, in other words, areas with the highest level of 
segregation. Integration proves to an important spatial variable that correlates well to social activities such as movement and interaction (Hillier 1996; Penn et al. 1998). Local integration (R3) measures accessibility up to three steps away and in terms of axial line, means a topological distance of three turns. There is evidence that suggest that integration at a local level correlates strongly with local pedestrian movement, meaning short trips to local destinations. It has also been shown to correlate with the movement of "locals" or "inhabitants", as compared to non residents and visitors who enter the spatial system from the outside (Hillier and Hanson 1984; Hillier 1996).

There are other notions in the space syntax method theory, for example, the notion of 'Connectivity', which measures the degree of intersection or one step possibilities of each axial line (Hillier 1996). The notion of 'intelligibility' is defined as the degree to what can be seen and experienced locally in the system (Hillier 1996). The 'intelligibility' value is calculated by the degree of linear correlation between connectivity and global integration value (Hillier and Hanson 1984). 'Synergy', denotes the degree of linear correlation between $\mathrm{R} 3$ and $\mathrm{Rn}$, which is used to mitigate the influence of system size (Hillier et al. 1993a).

This broadly describes the original formulation of space syntax. There have been significant developments beyond this, which I will consider later in this chapter. First though, I summarize some of the basic method's criticism.

\subsection{Critics of the space syntax method}

Space syntax methodology has been developed for more than 20 years since its first publication in 1984, and is widely adopted for the analysis of urban configuration structure and social economic prediction. However, controversies here also come with its success. The debates mainly focus on three aspects as follows: 
Firstly, its prediction ability is weak. Space syntax in its original topological formulation is criticized for only considering topology distance and ignoring other distance metric or 3D information about the urban system or building system, such as building height, land use, transportation infrastructure. For example, in terms of early building pattern analysis, Architect Lawrence (1990) and sociologist Edmund Leach (1978) pointed out that in a cultural context the syntactic arguments were insufficient to infer the social dynamics only with a building's floor plan. Similar sentiments were expressed by Osman and Mamoun (1996), who state that the boundaries between spaces are normally set by some kind of furniture rather than by physical barriers, and note that space syntax only models open floor plans.

Recently, Ratti (2004) criticized space syntax systematically, "How is it possible to tell so many things about the urban environment with such a limited amount of information that is, after having dismissed data such as the height of buildings and the size of streets". He also argued that topological distance is unacceptable, as the topological distance facing“ New Yorker living on Fifth Avenue between 111th and 112th Streets going to Central Park North round the corner (two changes of direction) is the same for him going to Columbus Circle....." (Ratti 2004). In fact, the Columbus Circle is much further away.

Steadman (2004) points out that the space syntax method seems to be problematic at the large urban scale. Movement along a straight but congested urban street is slow and requires the expenditure of energy. The urban traveller might be expected o choose the shortest metric distance for lower energy cost rather the fewest changes of direction. He also noted that the same value of integration cannot predict radically different flow volumes at two dates. Take Venice as an example. Its configuration has changed little from the $18^{\text {th }}$ century to the 21 st century and the integration value of all axial lines will have changed little over the period. It is doubtful, however, that pedestrian flows are correlated with the same integration over the period. 
However, Hillier and Penn(2004) disagrees with Ratti over the use of topological distance in spatial modelling instead of metric distance, insisting that when employing topology, the problem of boundary present no border effects. For instance, when city centres are not in the centre of geometry of the study area, high integration still occurs in the street segments near the functional center rather than at the center of your study area.

Porta et al.(2006) compared the performance of the prime approach and the dual approach in Ahmedabad, Vennice, Richmond, CA and Walnut Greek, CA. The first approach being a metric geographic network analysis method, and the latter the space syntax topology method. The conclusion they made was that the prime approach is in fact, vulnerable to the Border effect.

Secondly, space syntax is also criticized for the procedure it was in creating an axial map which has been alleged to be not objective. Although, the whole process should be based on the principle of 'longest and fewest' lines in the street network, several authors (Batty 2001; Batty and Rana 2002; Jiang and Claramunt 2002) showed that process of creation axial map is arbitrary, as it seems there is no formal evidence showing the unique set of axial lines for a space. This uncertainty would lead to inaccurate creation of the axial map, influencing analysis results. Hence it seems different user could generate different sets of axial map for the same application.

Hillier and Penn(2004)countered that the process of creating an axial map is not arbitrary and Carvalho and Penn (2004) used a statistical method for axial line lengths of thirty-six cities. They found that axial lines are statistically significant elements, while the errors associated with tracing them are not as significant as expected. Hillier argued that the reason for misunderstanding the issue of arbitrary lines is that critics thought axial maps depend on the prior construction of a unique convex map, which 
they do not, either practically or theoretically. He also presented an argument to show that theoretically there is only one correct graph of a road network.

Thirdly, Ratti (2004) argues that several inconsistencies when apply the axial map to illustrate similar the urban layouts, even there is a cross error for space syntax topology measurement.

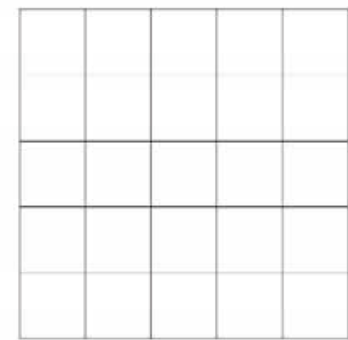

(a)

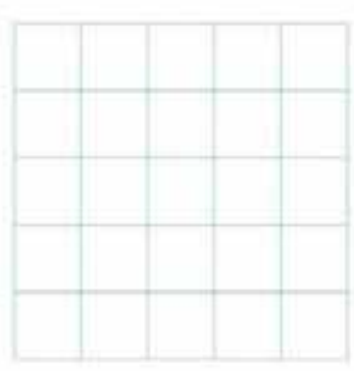

Integration value $=3.134$

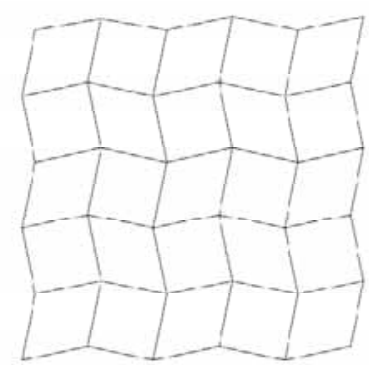

(b)

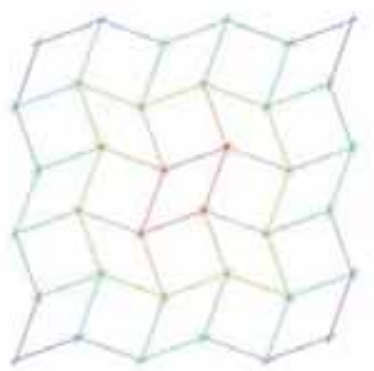

Integration value $=1.930$ (central) 0.919 (corner)

Figure 3.5 Value changes when deform the configuration

Source: (Ratti 2004)

In figure (3.5) for example, "a" is an orthogonal axial map, and "b" is a 'broken' or deformed one. It is seen that such similar configurations have such distinct integration value. Diagram (a) scores a uniform integration value 3.134, while the range of integration value in diagram (b) is between 0.919 (central segments) and 1.930 (peripheral segments).

Hillier and Penn (2004) noted that Ratti's results were correct, but that these two 
configurations are actually "syntactically" different, and thus, different values of Integration are reasonable. Diagram (b) is comprised of broken segments and Hillier argued that Ratti's inference conflicted with the topological issue.

Ratti (2004) also inferred that the procedure of axial map creation represented a discontinuity in the transformation from geometry to topology. Because at a certain angle and certain street width, axial lines of a deformed grid would pass through all blocks and create the same configuration as an orthogonal grid. As seen in figure (3.6), there are seven axial lines for the layout in the left diagram, but when the urban grid was deformed slightly in the right diagram, due to the 'fewest and longest' principle, three axial lines are sufficient to represent the layout - the same results for an orthogonal version of the grid.
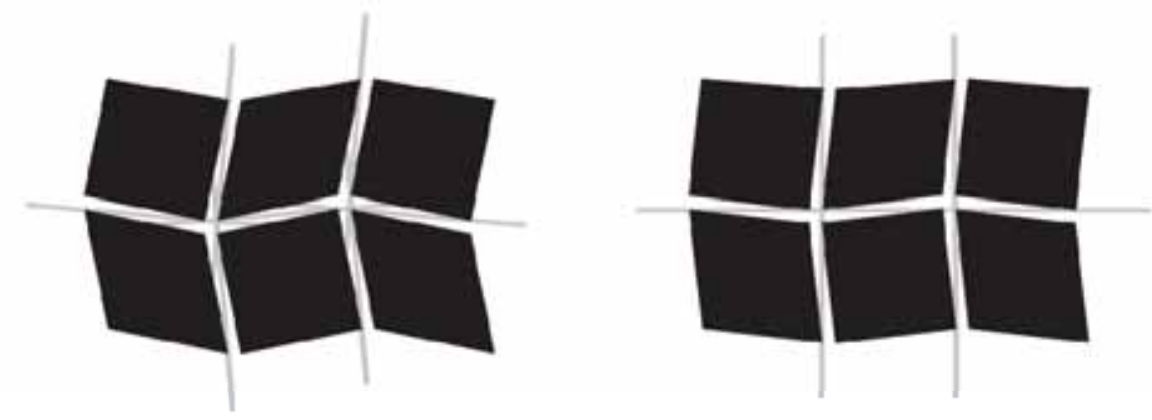

Figure 3.6Inconsistency of axial line

Source: (Ratti 2004)

Another inconsistency, or logical flow, identified by Ratti is the crossed-error implicit in space syntax topological methodology(Ratti 2004). As figure (3.7) shows, when two separated axial maps are linked with an extra axial line, the integration pattern changes dramatically, Ratti therefore concluded that the method is sensitive to choice of study boundary, as it influences the value of integration. 

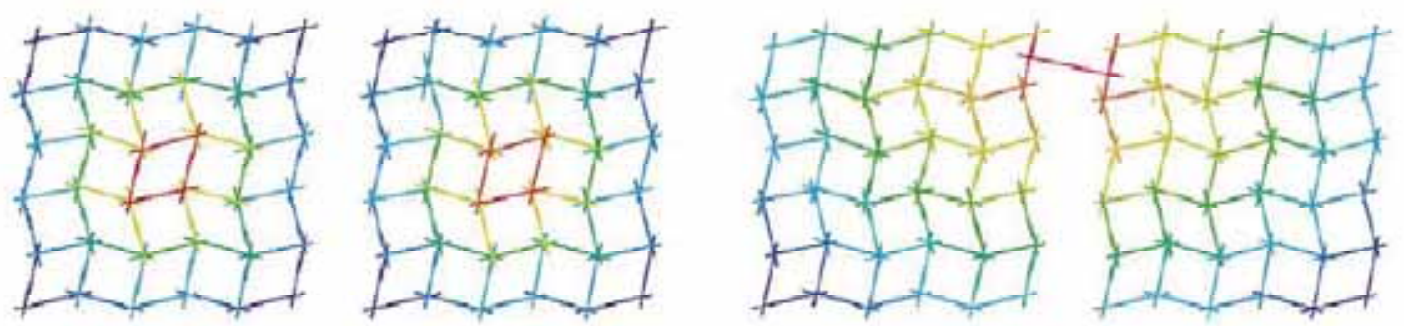

Figure 3.7Cross error for two axial line maps

Source: (Ratti 2004)

Hillier and Penn(2004) admitted that the phenomenon could happen in areal urban study. The most integrated part would shift from its central region to the region connecting the outside world. However, the notion of metric search radii can relieve the problem of edge-effect. By setting certain radii for the mean depth of the system though street segment. However, they shared Ratti's view that axial analysis does not model the real movement rate.

\subsection{Developments of space syntax theory}

Criticism has always been the driving force for development, Hillier and his colleges have improved the space syntax methodology by enhancing the specification of axial map theory to create unique axial line maps; as well as developing metric segment analysis and geometric segment analysis to deal with the inconsistencies stated by Ratti (2004) and others. Hillier and his colleagues assign space syntax measurements by different definitions of weighted distance, including shortest path (metric), least angle change (geometric), fewest turns (topological) weightings to relations between each segment and all others (Hillier and Iida 2005). Since then, space syntax has became a two dimensional measurement for spatial accessibility, which bring it closer to conventional traffic models. The detail developments are discussed below: 


\subsubsection{Unique axial line map}

Responding to the criticisms regarding the exact definition of axial lines, and its non-uniqueness, Hillier and Penn (2004) argued that theoretically there is only one correct axial line map. Furthermore, Turner et al. (2005) proposed an algorithmic solution of the axial map, which delivers a sound definition and methodology in order to obtain a unique map. The new approach is derived from the research done by Penn (1997), and his solution involves two steps. The first step is identical to the method Penn published in 1997 to generate all axial line maps, which includes the reduction of the lines starting with the longest line, see figure (a). The second step concerns retrieval of the complete axial line system by preserving the topological rings, as displayed in figure (b). A detailed account of the idea of topological rings, can be found in Peponis et al. (1998).
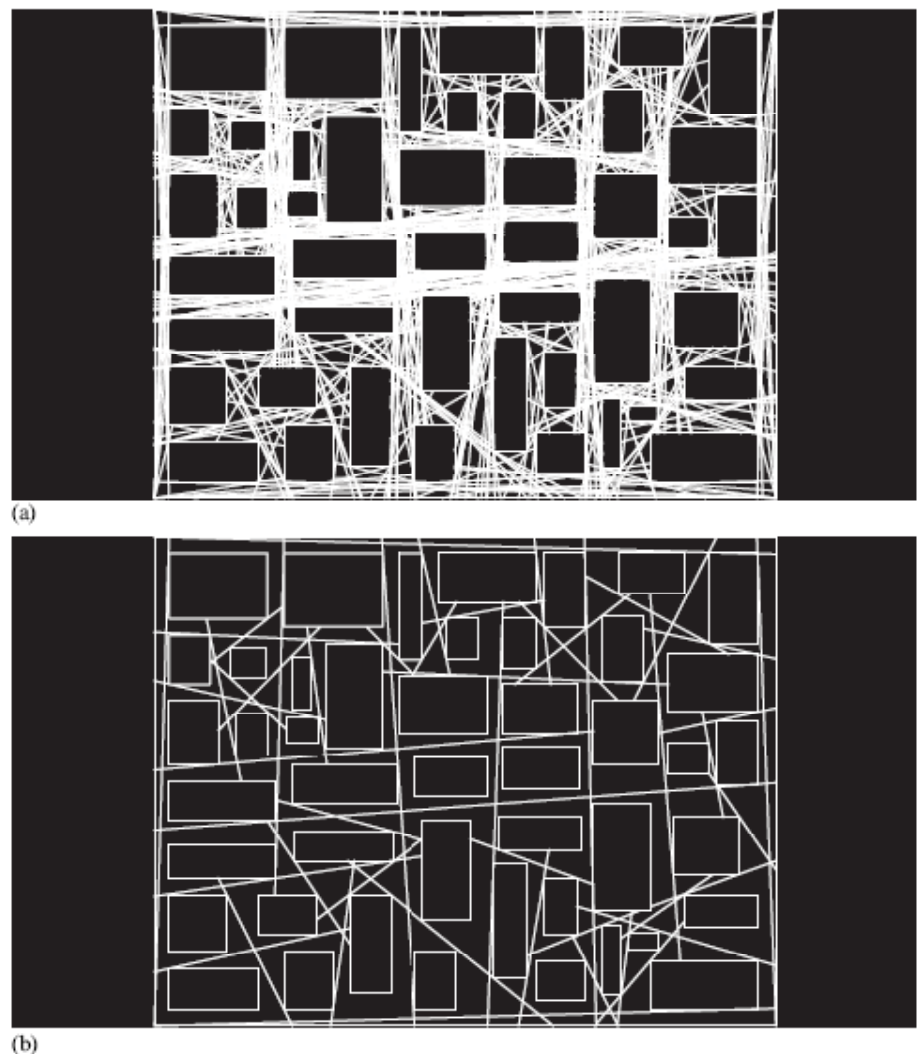

Figure 3.8 An algorithmic definition of the axial map Source: (Hillier and Penn 2004) 
Jiang and Liu (2009) introduced an alternative algorithm for identifying unique axial lines, named AxialGen. The function allows automatic generation of a unique axial map by selecting the least number of the longest visible lines (or axial lines) representing individual open space (convex space) in an urban environment. The substantial difference between AxialGen and Space syntax lies in their dissimilar first step solutions. AxialGen uses Isovist analysis retrieval, developed by Batty and Rana (2004), with an algorithm similar to Peponis's (1998). Figure (3.8) 'a' is a fictional spatial layout; and diagram 'b' shows the spaces divided into Voronoi regions of closed spaces. Taking any pointon the edge of the Voronoi region for isovist visibility analysis, creates a set of isovist ridges, and the longest isovist ridges in the isovist area can represent the whole isovist area, as seen in diagram 'c'. Diagram 'd' shows the final selected axial lines which compare the least number of longest visibility lines.

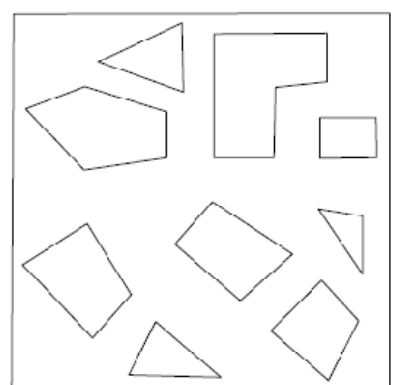

(a)

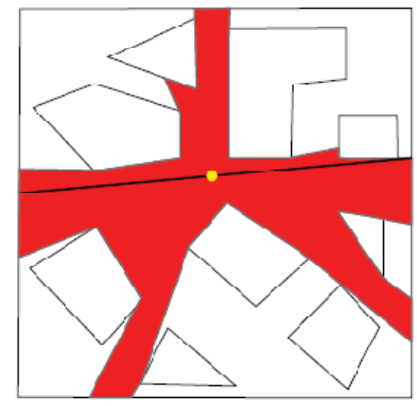

(c)

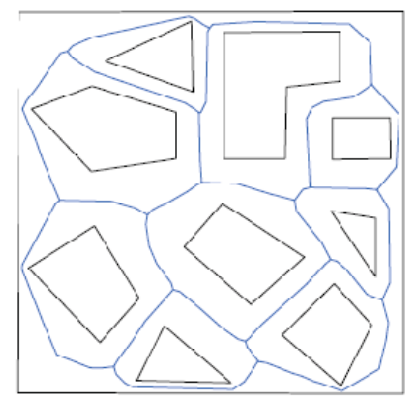

(b)

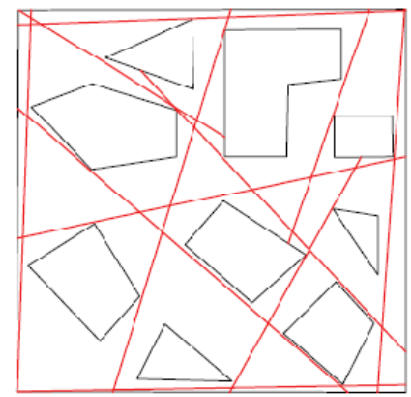

(d)

Figure 3.9Definition of axial line by AxialGen

Source:(Jiang and Liu 2009) 
Critics also point out the discussion of necessity of axial lines in space syntax, for example, Turner (2007) first raised the question "why not other representation?" and applied an angular segment analysis to compare road-centre line with axial line models of an urban road network. The results showed that the road-centre line with angular analysis had a stronger correlation with observed vehicular flows, implying that it is feasible to combined traditional transportation network analysis representations with the space syntax method, as it will be convenient to get a coherent cognition on the movement in the city.

\subsubsection{Segment Metric Radius measurement}

As Ratti already showed that there is a cross-error in space syntax topology analysis, when two axial map commutate each other, with the integration value changing dramatically. The radial segment analysis introduced by Hillierand Penn(2004) can avoid this error, as the metric radii can control the integration value, which depends on the size of the analysed area and avoiding edge effects. To do this, firstly, the axial line map is transformed into a segment line map, breaking down all axial lines by intersections and removing $25 \%$ of the overall length of line. This procedure can be carried out automatically through the application of the SSX 'Depthmap' software. Rather than topological accessibility, calculating the depth from a segment $\mathrm{x}$ to all other segment, the radii segment analysis for accessibility calculates the depth but within a fix metric radius (Turner 2007). So, if we know the size of our study area, we can define the exact radius we need to take so as to avoid edge effects.

Besides space syntax integration, space syntax brings a new notion of 'choice', also known as 'betweenness'. Betweenness is another centrality measurement popular used in the field of social network analysis. In space syntax theory, 'choice' measures of the flow through a space (Hillier et al. 1987). It captures how often, on average, a location may be used in journeys from all places to all others in the city. Locations 
that occur on many of the shortest paths between others (i.e., provide a strong choice) have higher betweenness than those that do not. Normally a main street has a high value whereas the choice value is a side-street off a main road has a lower volume. Thus a side street may have high integration but low choice.

\subsubsection{Angular segmentmeasurement}

Angular segment analysis deals with discontinuities, when geometric configuration changes slightly. Dalton (2003) argued with her British library hypothesis that people prefer to minimize the angular distance to their destination.
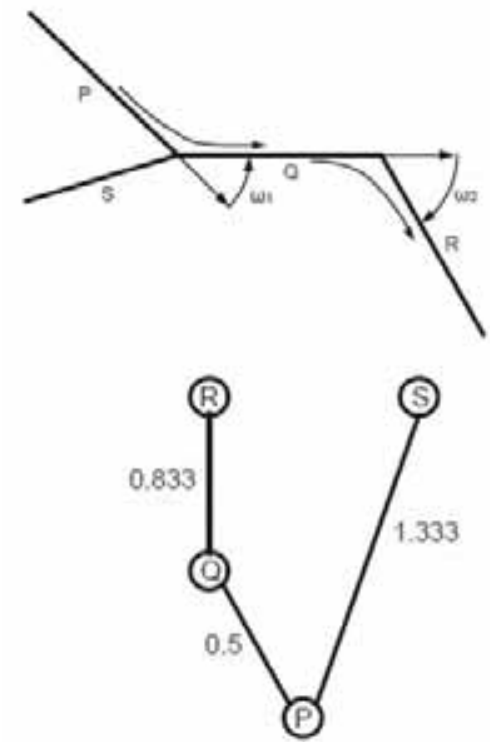

Figure 3.10Notion of angular cost

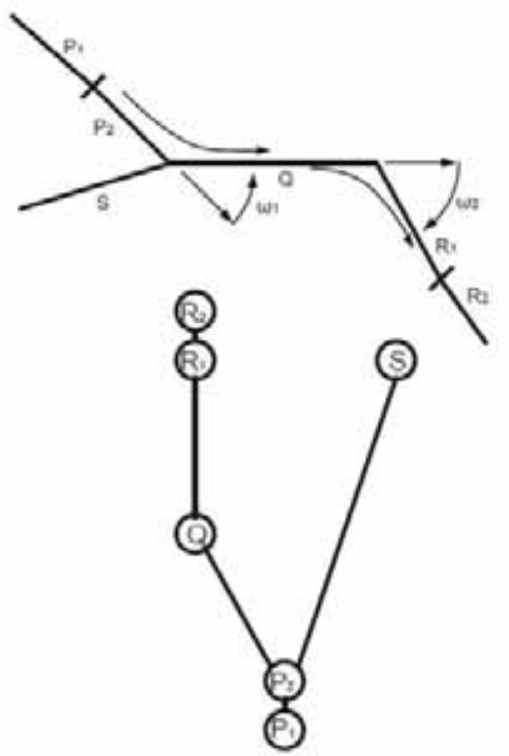

Source:(Turner 2007)

Figure (3.10) shows a simplified segmented axial map and its associated 'j-graph' of segments. Firstly, to calculate the depth to any location by lowest angular cost, we calculate the total angular turn from one segment to another segment via the shortest angular route,$d_{\theta}(\mathrm{x} ; \mathrm{y})$, where $\mathrm{x}$ is the starting segment and $\mathrm{y}$ the end segment of the path. Hillier and Iida's method (2005) assign a value in the range of 0 (no turn) to 2 ( $180^{\circ}$ turn) for each turn. So, in figure (3.10), the depth from segment $\mathrm{P}$ to segment $\mathrm{Q}$ 
is 0.5 (a turn of $45^{\circ}$ ) and the depth to segment $\mathrm{R}$ is 1.333 (a turn of $45^{\circ}$ followed by a turn of $60^{\circ}$, note that the direction of turn is immaterial, the turn angle is always positive).

By introducing alternative measures of distance: shortest path (metric), least angle change (geometric), and fewest turns (topological), to segments within any fix radius, with radius measured on metric, geometric and topological domains, space syntax methodology has become a more accurate and flexible tool for exploring a broad range of behaviour in the built environment.

\subsection{How urban morphology interacts with social economics phenomenon}

Although it emerged from architectural roots Space syntax can be classified as a location-based social network analysis method. It abstract the spatial accessibility from a complex urban configuration, describing how one street segment connects to others and produces indices that can be taken as a surrogate for distribution of people's movement within the urban layout. It is known that people have an innate ability to read or comprehend the meanings of different arrangements or layout of space, and react to physical infrastructure change (Hillier and Hanson 1984). However, traditional social economic and environmental studies ignore the spatial information contained in urban morphology, mainly considering people's tendency to seek accessibility for maximum benefits with minimum cost (e.g. distance, travel time, composite travel cost), as in traditional traffic models. Recently, some studies have connected space syntax to other academic fields, examining how the spatial accessibility contained in urban morphology impacts economic and social activities. For example, Vaughan and Penn (2006) used original census data to examine the relationship between immigrant clustering and street-level settlement patterns for the 
19th century Jewish immigrant quarters of Manchester and Leeds. They found there is strong negative relationship between the density of Jewish inhabitants and space syntax integration, indicating that Jewish immigrant chose the higher density areas, but with lower spatial integration streets. They sought opportunity but also sought refuge in back streets.

Hillier (2004) addresses the controversy about the relationship between crime and spatial design, finding a strong correlation between layout type and all kinds of crime. Traditional street patterns were found to be the best and the most 'modern' hierarchical layouts the worst, which indicate that rich and poor alike benefit from living in traditional streets. He also offers some simple design guidance: enhancing the accessibility and visibility of public space can positively influence crime rates. Nevertheless, the research draws a critical lesson that if security is to be enhanced, both global and local factors must be appropriate, otherwise, the security could not be increased. This illustrates one of the great strength of space syntax and related methods: the ability to examine what urban morphology effects at multiple scales (using multiple radii).

Nes and Rueb (2009) examine how the spatial layout of neighborhoods affect dwellers' behavior. They compared four different types of dwelling areas one with large social problems (Ondiep in Utrecht) and one without (Hof van Delft in Delft).Two other post-War urban areas were chosen - one with serious social problems (Oosterwei in Gouda) and one without (Ommoord in Rotterdam). They found that accessibility is highly related to anti-social behavior problems with a negative sign, indicating streets with high degree of inter-visibility and adjacent to main routes can contribute to create safe and vital dwelling areas.

Croxford et al. (1996) examine the relationship between street grid configuration and vehicular pollution, including Carbon Monoxide (CO), temperature, relative humidity, 
light level, and wind speed at six minute intervals. Finally, their research suggested that the relationship is strong, and urban pollution level could vary cross streets, even streets spatially close can have very different levels of pollution.

Barros et al. (2009) discuss the potential of space syntax as a tool for estimating traffic routes. In route analysis, space syntax achieved refined results when compared to SATURN (Simulation and Assignment of Traffic to Urban Road Networks). The results showed that the dual approach measuring the movement has better fitness than the regular geometrical approach, thence, it would be better suited in the transport field.

Other researchers have connected space syntax to the land value field, exploring the relationship between urban configuration and land use or housing price. For example, Brown (1999) noted that real estate analysts found local urban configuration land design to be important for the field of real estate but that they have not had the tools to build a strong theory. He compared two different types of shopping mall configuration using space syntax, and found that the reason why shopping mall number one was successful while shopping mall number two had failed was mainly due to configuration design.

Min et al. (2007) explored the relationship between the characteristics of location and land use, by a case study in Seoul. Their research showed that space syntax integration value has a high correlation with land value and the location propensity of industries. Similar findings are also found in the work of Kim and Sohn (2002).

These studies only focus on identifying positive or negative correlations between urban morphology and social economics activities, which can not be employed for prediction. Desyllas (2000) explored the relationship between urban street configuration and office rent patterns in Berlin by multiple regression analysis. A 
number of spatial and non-spatial independent variables (e.g. pre-letting time, unit floor space size and indexes and multipliers) were used in the determination of rent in his research. The results showed a shift in the pattern of location rent within the short period under analysis. Whereas the Western CBD was the peak area of location rents in1991-1994, the pattern by 1995-97 had shifted to Mitte. This was offered as direct evidence that prime location rents have reorganized around the new spatial structure of the city revealed by the measure of space syntax global integration. His finding is supported of the idea that spatial integration has an explanatory value for office rent.

Chiaradia et al. (2009) examine the relationship between street layout and residential property value by space syntax segment measurement (integration and choice). Their study applied Council Tax Band as a proxy for residential property value, and considered other attributes, including non-residential land uses (mainly retail), property size, building centered density, and age. Their findings showed that integration is much stronger than choice value, and integration is strongly and positively related to higher tax bands. When property size, age, and building ambient density are added, space syntax spatial locational variables are slightly weakened but still contribute to the property value. The likely reason is that betweeness has a high variance within a tax band while closeness tends to be more homogenous across locally continuous roads.

Matthews and Turnbull (2007) examined how street layout affects property value using space syntax network analysis for both east and west lake Washington. They used two methods to measure street layout indices: space syntax integration and ratio of segments/intersections. They found integration to be significant in both west and East samples using a 1400 feet walking distance radius, but the coefficient signs are opposite in the two samples. The ratio of segments/intersections had the same result. They concluded that the portion of house value contributed by street layout critically depends upon the context of the surrounding development pattern. Enström and 
Netzell (2008) also used space syntax methods in a hedonic price approach to test the urban street layout impact on commercial office rents in Stockholm. They approached space syntax integration and found that it showed a positive impact on office rent. More generally, street layout added additional explanatory power to hedonic models of office rent levels in a major city.

Several points need to be noted about space syntax use in causal modeling. Firstly, most studies connecting space syntax to other academic fields apply the space syntax topology measurement, which has been criticized by Ratii (2004) for inconsistencies. However, it seems that Ratii exaggerated the critics of space syntax. The main point of attack for space syntax theory by Ratti is that two quite similar axial maps could have two distinct results. In that case the inappropriate axial map creation would bias the analysis results. Indeed, the axial map is unique theoretically, which is proved by the works of Peponis, Wineman, Bafna, Rashid, Kim (1998), Turner, Penn and Hillier (2005) and Jiang and Liu (2009). Therefore, it is expected that two axial map should have two different results even they are similar, as the space interpreted by axial map is actually 'syntactically' different. Furthermore, regarding to the axial map edge-effect pointed out by Ratii (2004), that phenomenon that 'cross error' would happen in topology measurement, as it is only one dimension. Indeed, considering the notion of metric, the problem has been addressed (Hillier and Penn, 2004), which also imply that there is no instrinsical inconsistency of topology measurement for axial map, but it seems difficult to interpret in different urban system with a certain people's social activities. Therefore, metric segment and geometric analysis seems more advanced, as different metric radii could interpret different kind of interactions between built environment and social processes.

Secondly, correlation is not prediction, so space syntax should be supported by statistical data, accounting for other attributes in urban system, such as land-use, the composition of the population, the location of houses and hobs and services and so on 
(Ratti 2004). For example, location theory suggests that accessibility could be the most important determinant of land value or property value. Although there is strong correlation of urban form and property value, few studies address the question of whether urban from can be treated as a determinant of property value statistically.

Thirdly, the theory have not outline clearly whether the relationship between building environment and human societies could vary cross space or different culture context. However, there is broadly a cognition of spatial heterogeneity of human activities (Collinge 1996; Monn 2001).

\subsection{Summary}

In this chapter, I have briefly reviewed space syntax methodology, which is dual approachto network analysis, measuring the accessibility information contained in a model of urban morphology. Although some researchers have pointed to inconsistencies inits topological distance measurement, a series of improvement such as metric segment analysis and geometric angular cost segment analysis.

As such, these researches will approach the geometric angular segment analysis measurement of space syntax. The reasons for choosing this measurement are that as mentioned above, there are some inconsistencies for typology distance measurement, and metric segment analysis does not consider depth 'cost' between two axial lines according to their angle of incidence. Hillier and Iida (2005) found that angular segment analysis is efficient and robust for measuring pedestrian movement in a large area of London. In a city-wide level, both vehicular and pedestrian, users are assumed to be sensitive of the cost of angular of street layout, and making $135^{\circ}$ turn seems cost more than making $90^{\circ}$ turn. Furthermore, in these studies, I adopt Turner's suggestion (2007) on creating the axial map based on road central line. The space syntax axial map was created as follows: 
1. A vector urban road network map was prepared and imported into Mapinfo 9.5.

2. An axial line map was created based on the principle of road central lines.

3. The map was modified to take into account of connections in $2 \mathrm{D}$ that do not hold in 3D (for example 'intersections' involving viaducts and tunnels.)

4. Using confeego 1.0, space syntax accessibility values were calculated for each segment.

Two essential indices of space syntax will be applied for capturing the accessibility contained in urban configuration, namely, integration and choice. Their formulas (Turner 2007) are provided as follows:

\section{Radius integration}

Space syntax integration measures to what extent a segment is close to all others segments along the shortest depths (paths) of the network. Space syntax integration for a segment $i$ is defined as:

$$
\text { Integration }_{i}=\frac{(N-1) \sum_{i}^{N} \ell_{i}}{\sum_{j=1 ; j \neq i}^{N} d_{i, j} \ell_{i}}
$$

Where

$\mathrm{N}$ is the total number of segments in the network,

$d_{i j}$ is the shortest depths between segment $\mathrm{i}$ and $\mathrm{j}$.

$\ell_{i}$ is the search length though segment $i$

The integration value commonly measures how well connected or how close one node is to all other nodes within the search radius. If the integration value is high it means it has a high spatial accessibility, as it is easier to move from one segment to others. Integration has been widely been used in urban and regional analysis as a proxy for the cost of overcoming spatial separation within a network of road segments. 


\section{Radius Choice}

Space syntax choice is based on the idea that a segment is more central when it is traversed by a larger number of the shortest paths connecting all couples of segments in the network, which is defined as(Turner 2007):

$$
\text { choice }_{x}=\sum_{i=1}^{N} \sum_{j=1}^{N} \sigma^{\ell}(i, x, j) i \neq j
$$

Where:

$\sigma(i, x, j)$ is the number of shortest paths from segment $\mathrm{j}$ to $\mathrm{k}$ through $\mathrm{x}$, $\sigma^{\ell}$ isthe weighted sigma function

Using a social network analogue, choice is like the kind of prominence of a person who acts as an intermediary among a large number of other persons. The choice can demonstrate how movements go through the network within a specific radius, and many studies have used different scales of choice to simulate movements associated with different travel modes. Generally, a high value of choice implies high frequencies of movement passing through the segment, which is expected to have a negative impact on residential property value.

I believe the new development of space syntax could improve its measurement in explaining the interaction between urban spatial structure and socio-economic activities.

In addition, I have also reviewed the studies that connect space syntax to other social science fields, especially land value theory. Location is considered as an essential determinant of housing price distribution, but currently most researchers ignore the spatial information hidden in an urban street grid. Given that a number of researchers in the space syntax community have confirmed that there is a high correlation 
between urban morphology and housing price pattern, there would seem to be a fruitful research agenda explaining this further. Hence, in the next chapters, I explore both the static and dynamic relationship between urban morphology and housing price,supported by statistical data that accounts for other attributes in urban system. Specifically, I will look at (a) the basic relationship urban morphological measures of accessibility and house price, holding other price determinants statistically constant; (b) the use of space syntax in delineating housing market areas; and (c) the dynamic relationship between changes in systemic accessibility (through incremental network improvements) and house price. 


\section{Chapter Four:}

\section{Urban configuration and housing price}

\subsection{Introduction}

House price can be thought of as a buyer's evaluation of a bundle of intrinsic and extrinsic attributes of a housing unit, including structural, locational, neighborhood and environment attributes (Freeman III 1979a). Many researchers have examined the contribution to house price of specific sets of factors. Structural characteristics typicaly include building type, numbers of bedrooms, numbers of bathrooms, garden, heating, swimming pool and so on (Follain and Jimenez 1985; Sirmans et al. 2005). Locational variables attempt to model accessibility to various sources of either positive or negative externalities, such as distance to $\mathrm{CBD}$, local commercial centres, parks and pollution sources. Environmental characteristics have included air quality (Freeman III 1979) and noise pollution (Bateman and Executive 2001; Day et al. 2007), and distance to the open space (Bolitzer and Netusil 2000). Neighborhood characteristics (a subset of locational attributes) typically include quality of school (Dubin and Goodman 1982; Haurin and Brasington 1996; Downes and Zabel 2002; Debrezion et al. 2006), socio-economic and racial composition (King and Mieszkowski 1973; Harris 1999; Ioannides 2002).

Locational attributes are well known to be key determinants of price since they are not easy to change - generally much less so than structural attributes of a building. In early studies, many researchers used the monocentric urban bid rent model to structure their enquiry. In the monocentric model, accessibility to $\mathrm{CBD}$ is the major determinant of location-specific land values and site rents. Many theoretical and empirical studies are premised on the central idea of the "access-space-trade-off" model, which gives rise to house prices falling with increased distance from the city 
center (Alonso 1964; Muth 1969; Mills 1972). These early studies are considered seminal in urban theory building but have given way to more nuanced studies that better capture the multicentric and network geometry and topology of locational advantage (McDonald 1987). A variety of accessibility measuring devices has been adopted to capture the locational premium effect more sensitively than the purely Euclidean distance used in many studies on property value (Niedercorn and Ammari 1987; Hoch and Waddell 1993). Specific approaches include studies specifying accessibility index by travel time (Landau et al. 1981), job accessibility (Srour et al. 2002), railway infrastructure improvement (Henneberry 1998), and systemic street network connectivity (Matthews and Turnbull 2007; Enström and Netzell 2008).

In this chapter, I explore the intuitive hypothesis that important information about urban agglomeration externalities is contained in the urban street network grid and can be captured from accessibility indicators measured from a topological network model of the grid. Most hedonic models fail to take note of essential spatial information in the street layout: network connectivity, network distance, angular distance and so on, which in turn, have been shown to have a strong influence on pedestrian behavior, congestion and other influences on house price (Hillier and Hanson 1984b; Hillier 1996; Penn et al. 1998; Vaughan et al. 2005; Chiaradia et al. 2009).

I test this hypothesis using Cardiff as the study area. A model of a sub-section of the Cardiff street grid was modeled using the space syntax methodology and various measurements of network accessibility were taken from the model to form additional locational variables in a hedonic model of house prices. Furthermore, Garrett (2003) state the economic behavior depends upon the level of data aggregation, that is why the sign and significance of coefficient estimates from the regression using aggregated data differ from regressions using less aggregated data. In doing this I consider two level data aggregation, one is disaggregated data level, the other is aggregated by 
output area level, testing the following set of specific hypotheses:

1. Accessibility information derived from a topological network model improves hedonic model performance, compared to other locational data, in respect of:
a. \% of variation explained
b. distinguishing between positive and negative impacts of accessibility
c. spatial auto-correlation issues

2. The advantages hypothesized in (1) hold with both aggregate and disaggregate models

3. The explanatory power of network accessibility metrics varies with the shape of the study area.

In Section 2, I review previously published work relating to these issue. The hedonic model is presented in Section 3. The region and data are described in Section 4. Results are presented in Section 5, where we run 6 types of model: Model I (a) is a disaggregated hedonic model with conventional locational variables; Model I (b) is a disaggregated model with a full set of network accessibility variables (space syntax Integration and Choice measured at various radial scales in network space). Models I(c) is a set of 14 models each with a single pair of Integration and Choice measure, calculated at a particular radial scale in order to compare the efficacy of different scales of measurement. Models II (a), (b) and (c) are the same but using data aggregated to Census output areas rather than individual house units. Section 6 summarizes the findings and concludes.

\subsection{Locational information in hedonic models}

In access versus space trade-off models, transportation costs are traded off against land rents. Improvements in transportation infrastructure are assumed to reduce commuting costs via savings in commuting time, releasing more from a household budget to spend on land rent (Alonso 1964b; Muth 1969; Mills 1972). However, 
Alonso's (1964) monocentric model is clearly one pertaining to a particular historical phase in a city's evolution and does not adequately capture accessibility value where workplaces are not solely located in the city center(McDonald 1987), and where trips to work form a declining share of overall household travel. The polycentric nature of many housing-market areas makes one-dimensional separation measures like physical distance and travel time from a distinctly defined center problematic. The presence of multiple-worker households and multiple workplaces motivated the search for alternative separation measures, as Heikkila et al note:

"with multiple-worker households, multiple workplaces are common; given a high degree of residential mobility, sites offering accessibility to many employment nodes are more valuable because it is not very likely that successive owners will work in the same workplace" (1989, page 222).

The study by Heikkila et al found that distance to CBD had a very low t-value and an unexpected sign in a house price hedonic model of Los Angeles in 1980 and they concluded that the impact of workplace accessibility has been overemphasized. Richardson et al. (1990)note that the effect of distance to CBD may have declined, find in that the distance coefficient for Los Angeles CBD was significantly negative in a dataset for 1970. Waddell et al. (1993) emphasized the importance of including distance to secondary employment centers, and found both a strong and significant asymmetric CBD-gradient and strong local effects from non-CBD employment centers. More recently McMillen (2003)took an alternative repeat sales model to identify changes in housing price distance gradients for Chicago CBD and found the distance coefficients of Chicago CBD was insignificant in the early to mid 1980s, but that this situation had reversed by the 1990s. Orford (2002) specified a multilevel hedonic model to evaluate locational externalities, examining a range of locational effects on house prices. In terms of property-level externalities, he used distance to CBD, motorway exits, railway stations, shopping centers, and suburban employment 
centers. He also considered proximity to nonresidential land uses such as parks, schools, industry, commercial, local shops, recreational centers and cultural and educational centers. His results showed a complex geography of locational externality effects, with areas of positive and negative externalities in juxtaposition across highly localised areas.

There have been detailed studies of transport behavior and house price, for example, Tang (1975)which showed that savings in transportation cost tend to have a positive and significant impact on house prices in line with Alonso-style trade-off models. In general, the impacts of transportation on residential markets are related to availability of transport; transportation costs; travel time; and convenience of transport. Gibbons and Machin (2005) explored the effects on house prices of a transport innovation for households in London in the late $20^{\text {th }}$ century. They defined rail access in two ways: distance to a station and service frequency at the nearest station. Distance changes induced by the transport innovation were associated with price changes and the price effect was large compared to the monetary valuation of other local amenities. On the other hand, Adair et al. (2000)examined the relationship of housing price and accessibility in Belfast urban area. They adopted a traffic gravity model, calculating the accessibility index for locations to various opportunities by different types of vehicle, finding that the index was significant, but accounts for a very small percent of the variation in house prices. Specific physical housing attributes and socioeconomic variables appeared to be a lot more influential. An example of a more sophisticated accessibility index is found in Osland and Thorsen(2008), who utilized a hedonic model to confirm that gravity-based labor-market accessibility significantly contributes to housing prices. Significantly, he found that labor-market accessibility is not an adequate alternative to distance from the $\mathrm{CBD}$; illustrating that there are multiple attributes bundled into the concepts of accessibility. Webster notes the conventional distinction between general and specific accessibility, elaborating this into the idea of geometric (or topological) accessibility and economic accessibility 
and giving both explicit economic interpretations (Webster 2010).

Grid structure (street layout) is an attribute of a city that influences both general and special accessibility. It confers general demand potential by virtue of the number of people passing through a location and it confers specific demand potential by virtue of the locational configuration demands of specific land uses. Matthews and Turnbull (2007) examined how street layout affects property value using space syntax network analysis for both east and west lake Washington. They used two methods to measure street layout indices: space syntax integration (defined formally in the next section of this paper) and ratio of segments/intersections. They found integration to be significant in both west and East samples using a 1400 feet walking distance radius, but the coefficient signs are opposite in the two samples. The ratio of segments/intersections had same result. They concluded that the portion of house value contributed by street layout critically depends upon the context of the surrounding development pattern. Enström and Netzell (2008) also used space syntax methods in a hedonic price approach to test the urban street layout impact on commercial office rents in Stockholm. They calculated space syntax integration and found that it had a positive impact on office rent. More generally, street layout added additional explanatory power to hedonic models of office rent levels in a major city.

The accessibility information contained in an urban street layout model would seem, in principle, a suitable approach for measuring locational characteristics at a disaggregated level without a pre-defined map of or knowledge about attractiveness hot spots. This chapter explores this proposition that whether urban morphology has impact on housing price.

\subsection{Methodology}

Space syntax is a set of analytical tool for quantatifying and describing the interaction between human and building environment (Hillier and Hanson 1984). Space syntax 
measures the accessibility of urban street and building configuration, initially based on the assumption that people move to areas where they can see, describing how the street network is connected (Hillier et al. 1993b). As discussed in Chapter three, one substantive difference between space syntax and traditional geographic network analysis, is that space syntax is a dual approach, which identifies streets as nodes and intersections as links (Batty 2004).

I adopt the segment metric radii measurement of space syntax. for this study, considering Turner's suggestion (2007) creating the axial map based on road central line. The overall methodology involves (a), creating an axial line map of a portion of the city of Cardiff grid; (b) calculating space syntax spatial accessibility indicators for each section in the grid, using the 'Confeego' software (www.spacesyntax.org); (c)compiling house price data for the same area and linking it to grid sections; (d) selecting a hedonic model functional form; (e) organizing the data for regression; (f) conducting econometric tests, predicting house price on the basis of, inter alia, connectivity information derived from the network model. These steps are elaborated below.

\subsubsection{Space syntax spatial accessibility index}

Two important measures of spatial accessibility: (a)integration, also known as closeness, and (b) choice, used in this study have been described already in Chapter three.

In this study, I will measure integration and choice at different radius: $400 \mathrm{~m}, 800 \mathrm{~m}$ $1200 \mathrm{~m}, 1600 \mathrm{~m}, 2000 \mathrm{~m}, 2500 \mathrm{~m}, 3000 \mathrm{~m}, 4000 \mathrm{~m}, 5000 \mathrm{~m}, 6000 \mathrm{~m}, 7000 \mathrm{~m}, 8000 \mathrm{~m}$, $10000 \mathrm{~m}$ and global $\mathrm{Nm}$ (the entire city grid model). From published research, these can be associated with different uses of the grid, for example, $400 \mathrm{~m}-1200 \mathrm{~m}$ is walking scale and $1600 \mathrm{~m}-2000 \mathrm{~m}$ is cycling or running scale, while above $2500 \mathrm{~m}$ is 
automobile scale. Choice of radius also depends on the area of study. Larger study areas obviously require a wider range of radius. Through testing the different choice radii on housing price in out study area, I can imply what kind of grid-use activity can affect house prices.

\subsubsection{Hedonic regression model}

The hedonic price model, derived mostly from Lancaster's (1966) consumer theory and Rosen's (1974) model, posits that a property possesses a myriad of attributes that combine to form bundles of utility-affecting attributes for consumers. The implicit price of the housing attribute revealed in the regression coefficient is equal to people's willingness to pay for each individual characteristic. The price of the house then, is the sum of the implicit prices for the attributes that are contained in it. Thus, the hedonic price approach enables the possible influence of each of the many attributes on the house price to be tested and analyzed.

The hedonic price model employed in this study is specified following Orford (1999)'s work, exploring the locational externality for Cardiff housing market:

$$
\begin{aligned}
\operatorname{Ln}\left(\text { Price }_{i}\right)= & \beta_{0}+\beta_{1} * \operatorname{Ln}(F L)+\beta_{2} * \operatorname{Ln}(D)+\beta_{3} * S S X+\beta_{4} * D U_{-} N+\beta_{5} \\
& * D U_{-} D+\beta_{6} * D Y \_S E+\beta_{7} * D U_{-} T+\beta_{8} * D U_{-} T E+\beta_{9} * D U_{-} B C \\
& +\beta_{10} * D U_{-} C L+\beta_{11} * D U_{-} P S+\beta_{12} * D U_{-} C C+\beta_{13} * D U_{-} T T+\beta_{14} \\
& * Y 2000+\beta_{15} * Y 2001+\beta_{16} * Y 2002+\beta_{17} * Y 2003+\beta_{18} \\
& * Y 2004+\beta_{19} * Y 2005+\beta_{20} * Y 2006+\beta_{21} * Y 2007+\varepsilon_{i}
\end{aligned}
$$

Where,

Price $_{i}=$ Transaction price of residential property $i$;

$F L=$ floor area;

$\mathrm{D}=$ straight Distance to the city center; 
$S S X=$ accessibility of urban configuration: integration and choice at different radius;

DU_N =dummy variable for new building;

DU_D =dummy variable for detached house,

DU_SE = dummy variable for semidetached house;

DU_T $=$ dummy variable for terrace house;

DU_Te = dummy variable for tenure;

DU_BC = dummy variable for people live in blue collar communities;

DU_CL = dummy variable for people live in the city;

DU_PS = dummy variable for people are prospering suburbs;

DU_CC = dummy variable for people are constrained by circumstances;

DU_TT $=$ dummy variable for people are typical traits;

Y2000 = dummy variable for transaction year of property in 2000;

Y2001 = dummy variable for transaction year of property in 2001;

Y2002 = dummy variable for transaction year of property in 2002;

Y2003 = dummy variable for transaction year of property in 2003;

Y2004 = dummy variable for transaction year of property in 2004;

Y2005 = dummy variable for transaction year of property in 2005;

Y2006 = dummy variable for transaction year of property in 2006;

Y2007 = dummy variable for transaction year of property in 2007;

$\varepsilon_{i}=$ A random error, which expected to be normal distribution with a mean of zero as well as the variance of $\sigma_{\varepsilon}^{2}$

\subsection{Data and study area}

\subsubsection{Datasets}

The dataset employed in this study mainly consists of three parts. The spatial data on housing price and all structure characteristics, which comes from the land registry; socioeconomic status information from the 2001 census data; and urban configuration 
information from a space syntax model based on data from the latest UK ordnance survey (http://edina.ac.uk/digimap/).

Housing price and all structural characteristics are collected by the Land Registry on behalf of the UK government. The land registry department holds details of title, covenants, plot details for residential and commercial property in England and Wales since 1995. Purchasing the dataset from the registry, gives access to a service license to use the following data: the full address of the property (PAON, SAON, street, postcode, locality, town district, country), property size, transaction price for the property, date of transfer, property type (Detached, Semi, Terraced, Flat/ Maisonett), whether the property is new build or not, and whether the property is freehold or leasehold. In this study, the dataset has 16,867 transactions during a period 2001 to 2007 at building level. Compared with previous studies of Cardiff housing markets his is a large study, with Orford (1999) using 1500 transactions (using asking price from the estate agents). The dataset also includes data for dwelling type, gross internal floor area, numbers of bathrooms, numbers of bedrooms, central heating, age bands, and off street parking.

Neighborhood type variables in this study, the Grand Index will be used to demonstrate the socio-economic status of the output area, which is from the 2001 Census data (http://edina.ac.uk/ukborders/). According to the Output Area Classification (OAC), the Grand index is divided into seven groups: blue collar communities, city living, countryside, prospering suburbs constrained by circumstances, typical traits and multicultural.

Urban configuration data are derived from the Ordnance Survey, which is the national mapping agency of Great Britain, which provides accurate and up-to-date geographical data. This kind of data cannot be implemented directly in space syntax network analysis as the space syntax method requires a model of the urban street 
segments network based upon the axial line, which is drawn based on the road central line. Furthermore, the area for network analysis should be bigger than the study area, normally an extra $3000 \mathrm{~m}$. Pre-processing also needs to convert three-dimensional intersections like viaducts or tunnels into a syntax model. Having prepared the OS data in this way, confeego 1.0 (www.spacesyntax.org) was used to calculate the urban configuration spatial accessibility, generating accessibility indices for each segment.

The three datasets were combined through ArcMap. The straight line distance of each property to the city centre was also calculated, and segment accessibility values were attached as an attribute to each house based on nearest criteria.

\subsubsection{Study area}

Since the object of the study is to examine whether the accessibility of urban configuration grid impact on the housing price, I selected a test area suitable for our experiment. The selection was constraint by the dataset available. Cardiff is the capital of Wales, the largest city and with the highest population in Wales. According to recent estimates, the population of the unitary authority area is $324,800 \mathrm{~m}^{2}$. Orford (2000) points out that the housing submarkets of Cardiff are stratified by dwelling type, based upon 26 basic units or communities used by the city council for administrative purpose. 


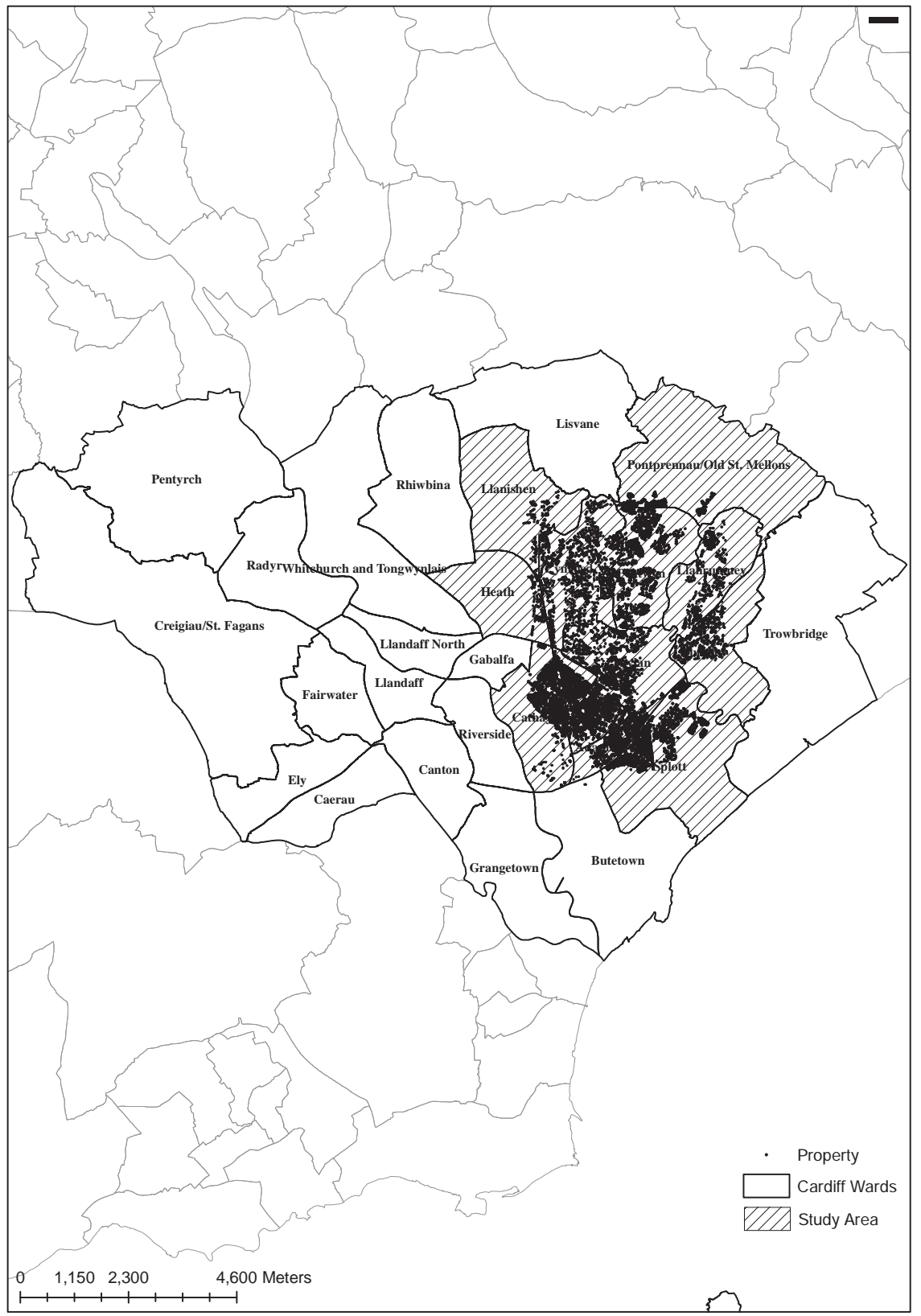

Figure 4.1Study area of Cardiff, UK

Source: author

The study area is limited to the eastern inner city wards of Cardiff which amount to almost including 11 wards: Cathays, Heath, Llanishan, Cyncoed, Penylan, Plasnewydd, Pentwyn, Llannumney, Rumney, Splott and Adamstown. The following table (4.1) illustrates the number of properties which meet the criteria of this study: 
Table 4.1The transaction number of each year

\begin{tabular}{cccccccccc}
\hline \hline Year & 2000 & 2001 & 2002 & 2003 & 2004 & 2005 & 2006 & 2007 & 2008 \\
\hline $\mathrm{N}$ & 1753 & 2198 & 2503 & 2228 & 2148 & 1737 & 2072 & 2057 & 174 \\
Total & & & & & 16867 & & & & \\
\hline
\end{tabular}

From the urban spatial layout perspective, there is a motorway (A48M) which divides the study area into two sample areas that differ greatly. They display significantly different social and development characteristics. The northern part of the study area is predominantly inhabited by family households and households who have access to private motor vehicles. The second sample area has high levels of residents who are students and are mainly depending on public transportation and walking. So based on this finding from the mapping, we can pre-define two spatial provisions: the northern and the southern part. It can be noted that earlier spatial approaches to housing market subdivision might have used this structure on its own.

For the aggregated hedonic models, the unit of measurement is output area, there being 322 census output areas in our study area. Figure 4.2 describes the average housing price at the aggregated level displayed in output areas. 


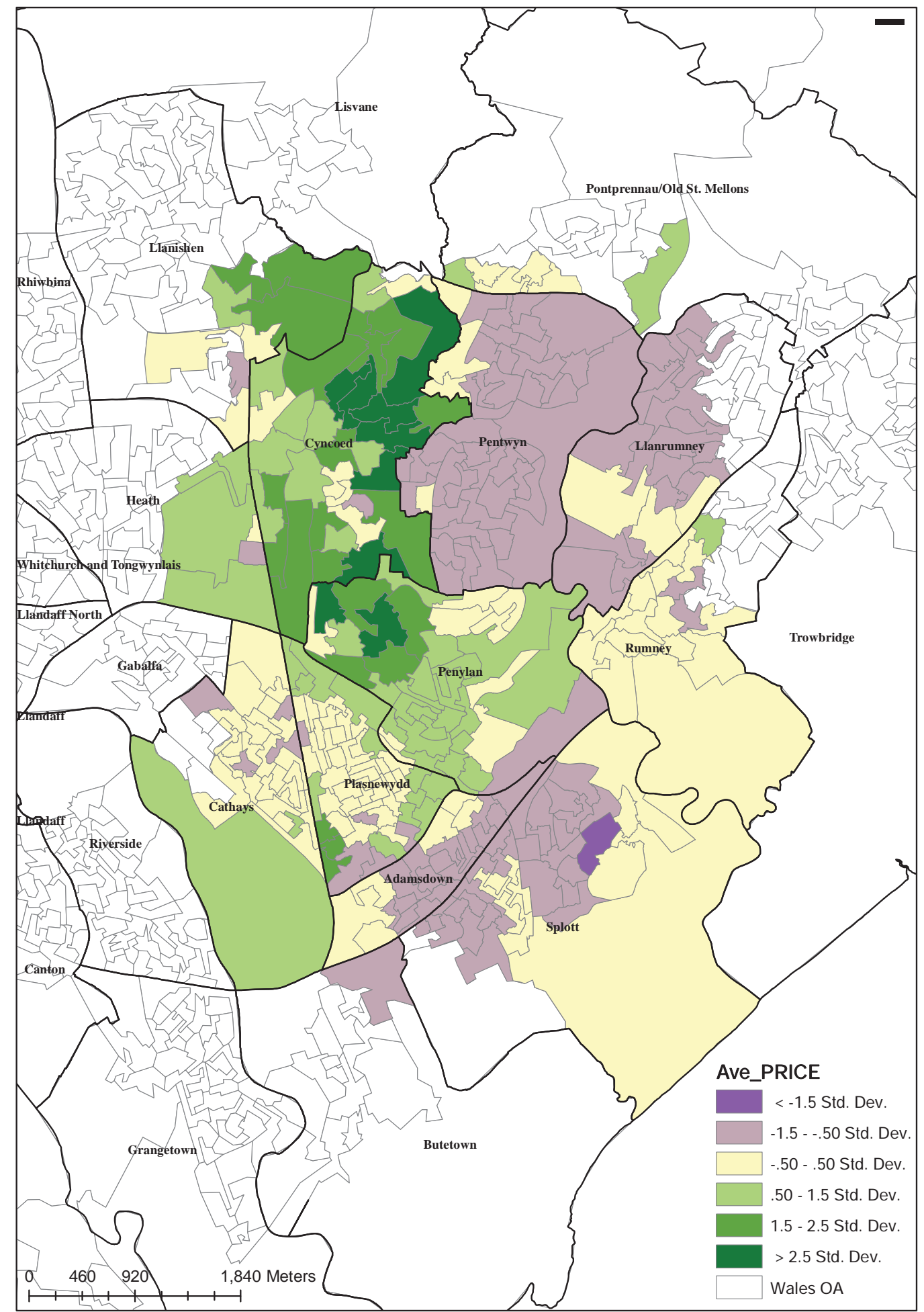

Figure 4.2The Std. Dev. of housing price in output area units

Source: author

\section{List of variables}


Fifty-five variables were prepared for the hedonic models: 21 dummy variables and 24 continuous variables.

Table 4.2Fifty-five variables and Description

\begin{tabular}{|c|c|c|c|c|c|c|c|c|c|}
\hline & Variables & $\mathrm{S}$ & $\mathrm{L}$ & $\mathrm{N}$ & $\mathrm{E}$ & SSX & $\mathrm{T}$ & Type & Description \\
\hline 1. & LN_FL & $\star$ & & & & & & Continuous & Ln floor area \\
\hline 2. & DU_N & $\star$ & & & & & & Dummy & New building $(Y e s=1 \mathrm{No}=0)$ \\
\hline 3. & DU_D & $\star$ & & & & & & Dummy & Detached House $(\mathrm{Yes}=1 \mathrm{No}=0)$ \\
\hline 4. & DU_SE & $\star$ & & & & & & Dummy & Semidetached House Yes $=1 \mathrm{No}=0$ \\
\hline 5. & DU_T & $\star$ & & & & & & Dummy & Terrace house Yes $=1 \mathrm{No}=0$ \\
\hline 6. & DU_F & $\star$ & & & & & & Dummy & Flat $\mathrm{Yes}=1 \mathrm{No}=0$ \\
\hline 7. & DU_TE & $\star$ & & & & & & Dummy & Tenure Freehold $=1$ Lease $=0$ \\
\hline 8. & DU_BC & & & $\star$ & & & & Dummy & Blue collar communities $\mathrm{Yes}=1 \mathrm{No}=0$ \\
\hline 9. & DU_CL & & & $\star$ & & & & Dummy & Lliving in the city $\mathrm{Yes}=1 \mathrm{No}=0$ \\
\hline 10. & DU_PS & & & $\star$ & & & & Dummy & Prosperious suburbs $\mathrm{Yes}=1 \mathrm{No}=0$ \\
\hline 11. & DU_CC & & & $\star$ & & & & Dummy & Constrained by Circumstances Yes $=1 \mathrm{No}=0$ \\
\hline 12. & DU_TT & & & $\star$ & & & & Dummy & Typical traits Yes $=1 \mathrm{No}=0$ \\
\hline 13. & DU_MU & & & $\star$ & & & & Dummy & Multicultural Yes $=1 \mathrm{No}=0$ \\
\hline 14. & LN_D & & $\star$ & & & & & Continues & straight-linedistance to CBD \\
\hline 15. & LN_D1 & & & & $\star$ & & & Continuous & straight line distance to Cardiff bay \\
\hline 16. & LN_D2 & & & & $\star$ & & & Continuous & straightline distance to Lake \\
\hline 17. & LN_D3 & & & & $\star$ & & & Continuous & straight line distance to hospital \\
\hline 18. & LN_D4 & & & & $\star$ & & & Continuous & straightline distance to Bute Park \\
\hline 19. & Y2000 & & & & & & $\star$ & Dummy & \\
\hline 20. & Y2001 & & & & & & $\star$ & Dummy & \\
\hline 21. & Y2002 & & & & & & $\star$ & Dummy & \\
\hline 22. & Y2003 & & & & & & $\star$ & Dummy & \\
\hline 23. & Y2004 & & & & & & $\star$ & Dummy & \\
\hline 24. & Y2005 & & & & & & $\star$ & Dummy & \\
\hline 25. & Y2006 & & & & & & $\star$ & Dummy & \\
\hline 26. & Y2007 & & & & & & $\star$ & Dummy & \\
\hline 27. & Y2008 & & & & & & $\star$ & Dummy & \\
\hline 28. & CH_R400M & & & & & $\star$ & & Continuous & SSX choice value at radius $400 \mathrm{~m}$ \\
\hline 29. & CH_R800M & & & & & $\star$ & & Continuous & SSX choice value at radius $800 \mathrm{~m}$ \\
\hline 30. & CH_R1200M & & & & & $\star$ & & Continuous & SSX choice value at radius $1200 \mathrm{~m}$ \\
\hline 31. & CH_R1600M & & & & & $\star$ & & Continuous & SSX choice value at radius $1600 \mathrm{~m}$ \\
\hline 32. & CH_R2000M & & & & & $\star$ & & Continuous & SSX choice value at radius $2000 \mathrm{~m}$ \\
\hline 33. & CH_R2500M & & & & & $\star$ & & Continuous & SSX choice value at radius $2500 \mathrm{~m}$ \\
\hline 34. & CH_R3000M & & & & & $\star$ & & Continuous & SSX choice value at radius $3000 \mathrm{~m}$ \\
\hline 35. & CH_R4000M & & & & & $\star$ & & Continuous & SSX choice value at radius $4000 \mathrm{~m}$ \\
\hline 36. & CH_R5000M & & & & & $\star$ & & Continuous & SSX choice value at radius $5000 \mathrm{~m}$ \\
\hline 37. & CH_R6000M & & & & & $\star$ & & Continuous & SSX choice value at radius $6000 \mathrm{~m}$ \\
\hline 38. & CH_R7000M & & & & & $\star$ & & Continuous & SSX choice value at radius $7000 \mathrm{~m}$ \\
\hline 39. & CH_R8000M & & & & & $\star$ & & Continuous & SSX choice value at radius $8000 \mathrm{~m}$ \\
\hline 40. & CH_R10000M & & & & & $\star$ & & Continuous & SSX choice value at radius $10000 \mathrm{~m}$ \\
\hline 41. & LOG_CH & & & & & $\star$ & & Continuous & SSX choice value at radius $\infty \mathrm{m}$ \\
\hline
\end{tabular}




\begin{tabular}{|c|c|c|c|c|}
\hline 42. & INT_R400M & $\star$ & Continuous & SSX integration value at radius $400 \mathrm{~m}$ \\
\hline 43. & INT_R800M & $\star$ & Continuous & SSX integration value at radius $800 \mathrm{~m}$ \\
\hline 44. & INT_R1200M & $\star$ & Continuous & integration value at radius $1200 \mathrm{~m}$ \\
\hline 45. & INT_R1600M & $\star$ & Continuous & integration value at radius $1600 \mathrm{~m}$ \\
\hline 46. & INT_R2000M & $\star$ & Continuous & integration value at radius $2000 \mathrm{~m}$ \\
\hline 47. & INT_R2500M & $\star$ & Continuous & SSX integration value at radius $2500 \mathrm{~m}$ \\
\hline 48. & INT_R3000M & $\star$ & Continuous & SSX integration value at radius $3000 \mathrm{~m}$ \\
\hline 49. & INT_R4000M & $\star$ & Continuous & SSX integration value at radius $4000 \mathrm{~m}$ \\
\hline 50. & INT_R5000M & $\star$ & Continuous & SSX integration value at radius $5000 \mathrm{~m}$ \\
\hline 51. & INT_R6000M & $\star$ & Continuous & SSX integration value at radius $6000 \mathrm{~m}$ \\
\hline 52. & INT_R7000M & $\star$ & Continuous & SSX integration value at radius $7000 \mathrm{~m}$ \\
\hline 53. & INT_R8000M & $\star$ & Continuous & SSX integration value at radius $8000 \mathrm{~m}$ \\
\hline 54. & INT_R10000M & $\star$ & Continuous & SSX integration value at radius $10000 \mathrm{~m}$ \\
\hline 55. & INT & $\star$ & Continuous & SSX integration value at radius $\propto m$ \\
\hline
\end{tabular}

\subsection{Empirical results}

\subsubsection{Street network analysis}

Finally, there are a total of 28 space syntax variables with 14 different search radii employed for testing the relationship between urban morphology and housing price, and their visualized maps are in Appendix -4. Generally, the red color in integration represents high connectivity, and the axial line with blue color indicate the connectivity of this street segment is low. With respect to the visualized choice maps, the high traffic flows is in red and segregated segments are colored with blue. It is noted that with the search radii increasing, the effect areas are enlarged, and geometry polycentric came out, shifted and regrouped which are found in both integration and choice maps. Furthermore, it is surprising to find that within a local level, the highest integration value is located in two streets, namely Crwys road - Albany road and Cowbridge east road, where are numbers of retail and commercial shops (Figure A4.1, 2, 3, 4, 9 and 10). However, when the search radii are above $3 \mathrm{~km}$, the highest integration value are shifted to the motorway (A48), shown in Figure A4.11, 12, 17, 18, 19 and 20. This implies that the travel preference of pedestrian and automobile on urban form are different, and it indicates maybe around $3 \mathrm{~km}$ is the optimal scale for all the people. 


\subsubsection{Disaggregated data}

I first explore the relationship between urban layout and house price at a disaggregated level. The descriptive statistics of the variables are shown in table 4.3. There are 16297 valid observations; 92\% buildings are not newly built; $79 \%$ of properties are freehold; terraced houses make up the largest portion at 52\%; and flats, the second largest portion. Only $5 \%$ of the output areas in the study are in the 'constrained by circumstances' neighborhood class; in contrast, $28 \%$ of output areas in the study area are in the 'typical traits' class. There are equal numbers of housing transactions from 2000 to 2007.

Table 4.3Descriptive Statistics for disaggregated dataset

\begin{tabular}{|c|c|c|c|c|c|}
\hline & $\mathrm{N}$ & Minimum & Maximum & Mean & Std. Deviation \\
\hline LN_PRICE & 17207 & 9.210 & 14.039 & 11.680 & 0.567 \\
\hline LN_FL & 16297 & 1.793 & 9.595 & 4.903 & 1.071 \\
\hline LN_D & 17207 & 12.804 & 12.821 & 12.811 & 0.004 \\
\hline LN_D1 & 17207 & 12.804 & 12.821 & 12.811 & 0.004 \\
\hline LN_D2 & 17207 & 12.804 & 12.821 & 12.811 & 0.004 \\
\hline LN_D3 & 17207 & 12.804 & 12.821 & 12.811 & 0.004 \\
\hline LN_D4 & 17207 & 12.804 & 12.821 & 12.811 & 0.004 \\
\hline DU_N & 17207 & 0.000 & 1.000 & 0.080 & 0.268 \\
\hline DU_D & 17207 & 0.000 & 1.000 & 0.100 & 0.300 \\
\hline DU_SE & 17207 & 0.000 & 1.000 & 0.210 & 0.407 \\
\hline DU_T & 17207 & 0.000 & 1.000 & 0.520 & 0.500 \\
\hline DU_F & 17207 & 0.000 & 1.000 & 0.170 & 0.375 \\
\hline DU_TE & 17207 & 0.000 & 1.000 & 0.790 & 0.411 \\
\hline DU_BC & 17207 & 0.000 & 1.000 & 0.110 & 0.310 \\
\hline DU_CL & 17207 & 0.000 & 1.000 & 0.270 & 0.446 \\
\hline DU_PS & 17207 & 0.000 & 1.000 & 0.150 & 0.354 \\
\hline DU_CC & 17207 & 0.000 & 1.000 & 0.050 & 0.208 \\
\hline DU_TT & 17207 & 0.000 & 1.000 & 0.280 & 0.449 \\
\hline DU_MU & 17207 & 0.000 & 1.000 & 0.150 & 0.353 \\
\hline Y2000 & 17207 & 0.000 & 1.000 & 0.110 & 0.309 \\
\hline Y2001 & 17207 & 0.000 & 1.000 & 0.130 & 0.340 \\
\hline Y2002 & 17207 & 0.000 & 1.000 & 0.150 & 0.359 \\
\hline
\end{tabular}




\begin{tabular}{|c|c|c|c|c|c|}
\hline Y2003 & 17207 & 0.000 & 1.000 & 0.130 & 0.339 \\
\hline Y2004 & 17207 & 0.000 & 1.000 & 0.130 & 0.332 \\
\hline Y2005 & 17207 & 0.000 & 1.000 & 0.100 & 0.301 \\
\hline Y2006 & 17207 & 0.000 & 1.000 & 0.120 & 0.328 \\
\hline Y2007 & 17207 & 0.000 & 1.000 & 0.120 & 0.321 \\
\hline Y2008 & 17207 & 0.000 & 1.000 & 0.010 & 0.099 \\
\hline CH_400M & 17207 & 0.000 & 3.183 & 1.483 & 0.923 \\
\hline INT_400M & 17207 & 0.000 & 95.898 & 29.479 & 16.135 \\
\hline CH_800M & 17207 & 0.000 & 3.879 & 2.152 & 1.277 \\
\hline INT_800M & 17207 & 0.000 & 194.551 & 68.402 & 40.154 \\
\hline CH_1200M & 17207 & 0.000 & 4.464 & 2.510 & 1.485 \\
\hline INT_1200M & 17207 & 11.928 & 350.988 & 120.269 & 67.933 \\
\hline $\mathrm{CH} \_1600 \mathrm{M}$ & 17207 & 0.000 & 4.961 & 2.745 & 1.626 \\
\hline INT_1600M & 17207 & 16.489 & 482.002 & 181.727 & 97.358 \\
\hline CH_2000M & 17207 & 0.000 & 5.287 & 2.912 & 1.721 \\
\hline INT_2000M & 17207 & 26.903 & 576.524 & 246.244 & 125.338 \\
\hline CH_2500M & 17207 & 0.000 & 5.570 & 3.069 & 1.817 \\
\hline INT_2500M & 17207 & 30.971 & 719.865 & 327.692 & 155.646 \\
\hline CH_3000M & 17207 & 0.000 & 5.789 & 3.195 & 1.891 \\
\hline INT_3000M & 17207 & 44.303 & 825.042 & 411.021 & 181.137 \\
\hline CH_4000M & 17207 & 0.000 & 6.155 & 3.389 & 2.004 \\
\hline INT_4000M & 17207 & 77.035 & 1044.480 & 584.539 & 219.047 \\
\hline CH_5000M & 17207 & 0.000 & 6.430 & 3.529 & 2.091 \\
\hline INT_5000M & 17207 & 141.572 & 1317.720 & 763.448 & 254.955 \\
\hline CH_6000M & 17207 & 0.000 & 6.655 & 3.634 & 2.161 \\
\hline INT_6000M & 17207 & 251.813 & 1604.220 & 944.528 & 281.435 \\
\hline $\mathrm{CH}_{-} 7000 \mathrm{M}$ & 17207 & 0.000 & 6.887 & 3.719 & 2.209 \\
\hline INT_7000M & 17207 & 359.996 & 1793.710 & 1114.311 & 298.407 \\
\hline CH_8000M & 17207 & 0.000 & 7.058 & 3.778 & 2.249 \\
\hline INT_8000M & 17207 & 441.707 & 1939.790 & 1248.791 & 308.140 \\
\hline CH_10000M & 17207 & 0.000 & 7.273 & 3.834 & 2.295 \\
\hline INT_10000M & 17207 & 616.409 & 2107.210 & 1412.711 & 292.122 \\
\hline LOG_CH & 17207 & 0.000 & 7.567 & 3.834 & 2.337 \\
\hline INT & 17207 & 858.122 & 2150.760 & 1521.650 & 245.688 \\
\hline Valid N (listwise) & 16297 & & & & \\
\hline
\end{tabular}

Two models were run: with and without the spatial accessibility indicators. To specify the latter model, a correlation test was undertaken (Annex One), which found that all locational variables and environmental variables such as the distance to the $\mathrm{CBD}$ and distance to the major park, are highly correlated. This is probably due to the scale of the research area, which is small, I therefore decided to only consider the locational 
attributes: straight distance to the city center. Dummies were created by suppressing "flat" and "multicultural" categories of the respective house-type and socioeconomic neighborhood type variables.

Model I (a) uses the traditional hedonic variables including structure, neighbourhood, environmental variables and year of sale attributes (Table 4.4)

The adjusted R-square is 0.615 . The log likelihood is minus 6184.150 , the Akaie info criterion is $0.761631, \mathrm{~F}$ is 1241.266 , and the probability of the F-test is 0.000 . I conclude that the model fits the data well and that each independent variable is significantly linear. As we expect, distance to the CBD has a negative relationship with house price; 'blue collar community' is also negatively related to the house price. All independent variables have a significant effect on housing price at a $5 \%$ confidence level, with all VIFs below ten. Model I's residual "White-test" results (table 4.5) shows that the $\mathrm{N}^{*}$ adjust $\mathrm{R}$-square is bigger than Chi-square (21), indicating heteroscedasticity, so the prediction of the model is poor. Moran's I (table 4.6) is 0.239, with a Z-score of $87.986(\mathrm{P}=-0.000)$ and there exist a significantly positive correlation, indicating the presence of a high value housing cluster and a low value housing cluster. I can imply the coefficients of each attributes are unreliable, leading to over estimation.

Model I (b) includes all 28 space syntax spatial accessibility variables (Table 4.4). The adjusted R-square is 0.654 and the log likelihood is -5303.758 . The Akaike info criterion is 0.657 and the F-value is 642.632 . Variation explained thus increased by $6.4 \%$ and the log likelihood increased by $14.23 \%$. Additionally, the Akaike info criterion rose by $13.75 \%$ and the sum of squared residuals decreased by 10.24\%.Model I (b) with urban configuration information therefore has higher explanatory power than model I (a). All variables in model I (b) are significant except DU_BC ('Blue collar neighbourhood'). This may be because Blue collar class people are more sensitive to accessibility. Distance to CBD has become positive, but with a 
weak standardized coefficient $(0.081)$. This suggests that once systemic network accessibility is accounted for, distance to CBD becomes a marginal influence, exerting a negative effect by virtue of the congestion associated with it. All variables in model I(a) have larger VIFs than in model I(b) but are still under 10, indicating that there is some interaction between physical attributes, socio- economic attributes and locational attributes, e.g. higher income people purchase larger houses farther from the city center but with high accessibility. Some of the network accessibility variables have positive coefficients and others negative, with similar P-values for all. As expected, most VIFs for the accessibility variables are over 10, indicating strong multicolinearity in model I(b). As discussed, the multicoliniarity will not affect the model's accuracy of prognosis, but the coefficients and the sign of coefficients should not be considered reliable. The White test for model I (b) (table 4.5) shows that $\mathrm{N}^{*}$ adjust R-square $>$ Chi square (48), which indicates heteroscedasticity in the residual. The OLS is no longer the best estimation and the model has lost its ability to predict. The z score of Moran's I in model I (b) (table 4.6) is 47.514 ( $p=0.000)$, which tell us there is still positive spatial autocorrelation in the residuals of the model. However, the $z$ score is smaller than in model I (a), with a $42.59 \%$ improvement, indicating that the space syntax variables ease the spatial autocorrelation effect and they add extra information in differentiating variation between the sample house price values.

Notwithstanding the slight reduction in some aspects of the model's reliability, it is still possible to conclude that the space syntax spatial accessibility variables have improved the estimation of house prices. I can say that urban layout significantly affects housing price and that when the configuration effect of the urban grid is taken into account, other cruder measures of accessibility (distance to CBD) are shown to have weaker and counter intuitive effects on price. 
Table 4.4Regression results of Model I (a) and (b)

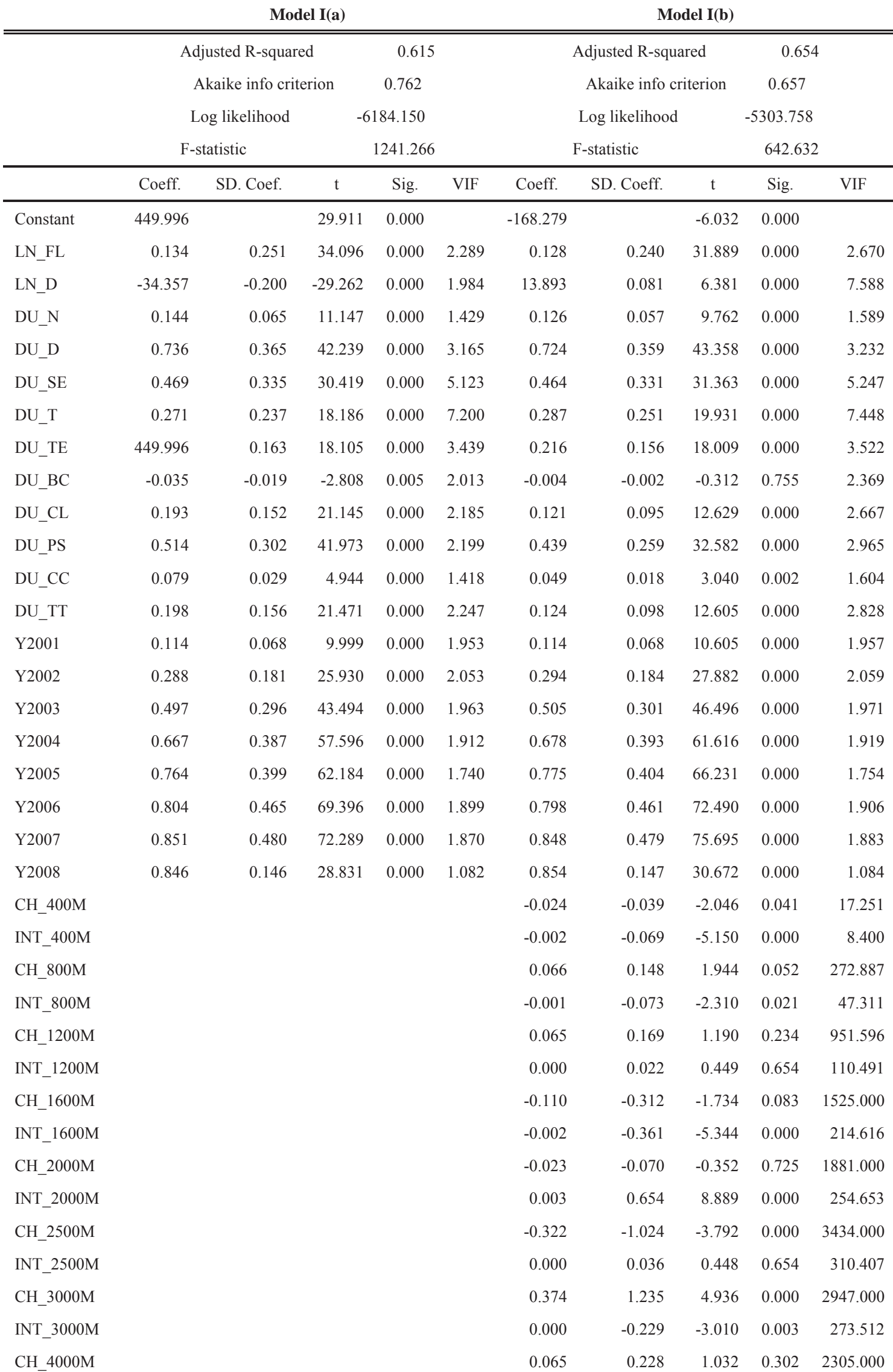




\begin{tabular}{|c|c|c|c|c|c|}
\hline INT_4000M & 0.000 & 0.116 & 1.743 & 0.081 & 209.872 \\
\hline $\mathrm{CH} \_5000 \mathrm{M}$ & -0.010 & -0.035 & -0.118 & 0.906 & 4110.000 \\
\hline INT_5000M & -0.001 & -0.629 & -8.875 & 0.000 & 236.375 \\
\hline CH_6000M & 0.063 & 0.237 & 1.014 & 0.311 & 2575.000 \\
\hline INT_6000M & 0.000 & 0.081 & 0.946 & 0.344 & 342.870 \\
\hline $\mathrm{CH}_{-} 7000 \mathrm{M}$ & -0.052 & -0.200 & -0.800 & 0.424 & 2936.000 \\
\hline INT_7000M & 0.002 & 0.909 & 9.800 & 0.000 & 405.171 \\
\hline CH_8000M & -0.015 & -0.059 & -0.220 & 0.826 & 3337.000 \\
\hline INT_8000M & 0.000 & -0.053 & -0.661 & 0.509 & 299.841 \\
\hline CH_10000M & -0.021 & -0.084 & -0.420 & 0.674 & 1880.000 \\
\hline INT_10000M & 0.001 & 0.444 & 7.866 & 0.000 & 150.178 \\
\hline LOG_CH & -0.046 & -0.188 & -3.163 & 0.002 & 165.544 \\
\hline INT & -0.001 & -0.590 & -15.176 & 0.000 & 71.220 \\
\hline
\end{tabular}

Table 4.5White test for Model I (a) and (b)

\begin{tabular}{|c|c|c|c|}
\hline \multicolumn{2}{|c|}{ Model I (a) } & \multicolumn{2}{|c|}{ Model I (b) } \\
\hline $\mathrm{N}=16297$ & Adjust R-square 0.019 & $\mathrm{~N}=16297$ & Adjust R-square \\
\hline $\mathrm{N}^{*}$ Adjust R-Square $=317.0412$ & Chi-square $(21)=32.671$ & $\mathrm{~N}^{*}$ Adjust R-Square $=395.968$ & Chi-square $(48)=65.171$ \\
\hline \multicolumn{2}{|c|}{ N*Adjust R-Square > Chi-square(21) } & \multicolumn{2}{|c|}{ N*Adjust R-Square > Chi-square(21) } \\
\hline
\end{tabular}

Table 4.6Global Moran's I for Model I (a) and (b)

\begin{tabular}{|c|c|c|c|c|c|c|c|}
\hline \multicolumn{4}{|c|}{ Model I (a) } & \multicolumn{4}{|c|}{ Model I (b) } \\
\hline Moran's I index: & 0.239 & Expected Index: & -0.000 & Moran's I index: & 0.129 & Expected Index: & -0.000 \\
\hline Variance: & 0.000 & Z Score: & 87.986 & Variance: & 0.000 & Z Score: & 47.514 \\
\hline p-value: & 0.000 & & & p-value: & 0.000 & & \\
\hline
\end{tabular}

I move on to look at the explanatory power of integration and choice measures at different radii in order to find out at what spatial scale the space syntax accessibility measures give most explanation, and it will imply us what kind people's activities is most associated with housing price in study area.

Each radius variable was put separately into model I(c). Table4.7 shows the comparison of $\mathrm{T}$ and $\mathrm{P}$ values for choice and integration at each spatial scale. VIF 
scores for each of the 14 versions of Model I (c) are within the desired range. I conclude from the table that choice and integration measures are significant whatever the spatial scale at which they are measured. This suggests that the volume of network flows and the convenience of connectivity at any point in the urban road network both significantly affect house price. The fact that all radii are significant suggests either that they all capture a similar accessibility effect or that they capture scale-specific effects that substitute for each other.

Choice has a negative relationship and integration a positive relationship with house price. This is as expected, since choice indicates likelihood of congestion and integration indicates ease of access to all other places within the radially-curtailed network section. In this way, the two network metrics neatly differentiate positive and negative network externalities. More can be said by examining the pattern of T-values. The change in T-value for integration rises from 2.773 to a peak of 28.163 at a radius of $7000 \mathrm{~m}$ and then falls again to 20.556.for a raidally unconstrained model (Figure 3.5). There is also a minor peak between $2000 \mathrm{~m}$ and $3000 \mathrm{~m}$. An opposite trend of T-values is observed for choice: first decreasing and then starting to rise at -8.374 , the lowest point being reached at radius of $7000 \mathrm{~m}$ and having a small negative fluctuation between $2000 \mathrm{~m}$ and $3000 \mathrm{~m}$. The adjusted $\mathrm{R}$ square values also peak at $7000 \mathrm{~m}$.

I may therefore conclude that for the study area, house prices seem to be most sensitive to network accessibility metrics measured at a radius of $7000 \mathrm{~m}$ and that this is true for negative and positive road network externalities. $7 \mathrm{KM}$ is the spatial scale that people use automobile within 10-15 mins. This indicates peoples' wiliness to pay extra only within a 10-15 mins driving distance to anyplace. Walking distance accessibility (400m-1200m) has less impact on housing price, but may be powerful in subsamples within smaller areas. Note that with radius increases the explanatory power of both integration and choice changes systematically, indicating that empirically, space syntax metric measurement is more accurate than the conventional 
geometric locational measurement. Network configuration becomes less influential beyond the $7000 \mathrm{~m}$ scale as it extends into ex-urban areas and the parts of the city the other side of the CBD, which indicates once more that beyond this distance there is no more city-scale externality effects captured in housing prices. This shows that optimal radius for measuring network accessibility is related to the size and shape of the study area.

Table 4.7 Model I (c): Different Radii - T value comparisons

\begin{tabular}{|c|c|c|c|c|c|c|c|c|c|c|}
\hline & \multicolumn{2}{|c|}{$400 \mathrm{M}$} & \multicolumn{2}{|c|}{$800 \mathrm{M}$} & \multicolumn{2}{|c|}{$1200 \mathrm{M}$} & \multicolumn{2}{|c|}{$1600 \mathrm{M}$} & \multicolumn{2}{|c|}{$2000 \mathrm{M}$} \\
\hline & $\mathrm{CH}$ & INT & $\mathrm{CH}$ & INT & $\mathrm{CH}$ & INT & $\mathrm{CH}$ & INT & $\mathrm{CH}$ & INT \\
\hline $\mathrm{T}$ & -2.314 & 2.773 & -4.627 & 11.343 & -5.98 & 15.42 & -7.332 & 19.024 & -8.536 & 23.439 \\
\hline Sig. & 0.021 & 0.006 & 0 & 0 & 0 & 0 & 0 & 0 & 0 & 0 \\
\hline \multirow[t]{3}{*}{ Ad- $R^{2}$} & \multicolumn{2}{|c|}{0.615} & \multicolumn{2}{|c|}{0.618} & \multicolumn{2}{|c|}{0.621} & \multicolumn{2}{|c|}{0.624} & \multicolumn{2}{|c|}{0.628} \\
\hline & \multicolumn{2}{|c|}{$2500 \mathrm{M}$} & \multicolumn{2}{|c|}{$3000 \mathrm{M}$} & \multicolumn{2}{|c|}{$4000 \mathrm{M}$} & \multicolumn{2}{|c|}{$5000 \mathrm{M}$} & \multicolumn{2}{|c|}{$6000 \mathrm{M}$} \\
\hline & $\mathrm{CH}$ & INT & $\mathrm{CH}$ & INT & $\mathrm{CH}$ & INT & $\mathrm{CH}$ & INT & $\mathrm{CH}$ & INT \\
\hline $\mathrm{T}$ & -8.416 & 24.836 & -8.139 & 24.609 & -7.367 & 22.2 .8 & -7.066 & 21.71 & -0.845 & 25.236 \\
\hline Sig. & 0 & 0 & 0 & 0 & 0 & 0 & 0 & 0 & 0 & 0 \\
\hline \multirow[t]{3}{*}{ Ad- $R^{2}$} & \multicolumn{2}{|c|}{0.629} & \multicolumn{2}{|c|}{0.629} & \multicolumn{2}{|c|}{0.627} & \multicolumn{2}{|c|}{0.626} & \multicolumn{2}{|c|}{0.63} \\
\hline & \multicolumn{2}{|c|}{$7000 \mathrm{M}$} & \multicolumn{2}{|c|}{$8000 \mathrm{M}$} & \multicolumn{2}{|c|}{$10000 \mathrm{M}$} & \multicolumn{2}{|c|}{ Infinite } & & \\
\hline & $\mathrm{CH}$ & INT & $\mathrm{CH}$ & INT & $\mathrm{CH}$ & INT & $\mathrm{CH}$ & INT & & \\
\hline $\mathrm{T}$ & -10.04 & 28.163 & -9.801 & 26.805 & -8.487 & 23.18 & -8.374 & 20.556 & & \\
\hline Sig. & 0 & 0 & 0 & 0 & 0 & 0 & 0 & 0 & & \\
\hline Ad- $R^{2}$ & \multicolumn{2}{|c|}{0.633} & \multicolumn{2}{|c|}{0.632} & \multicolumn{2}{|c|}{0.628} & \multicolumn{2}{|c|}{0.625} & & \\
\hline
\end{tabular}




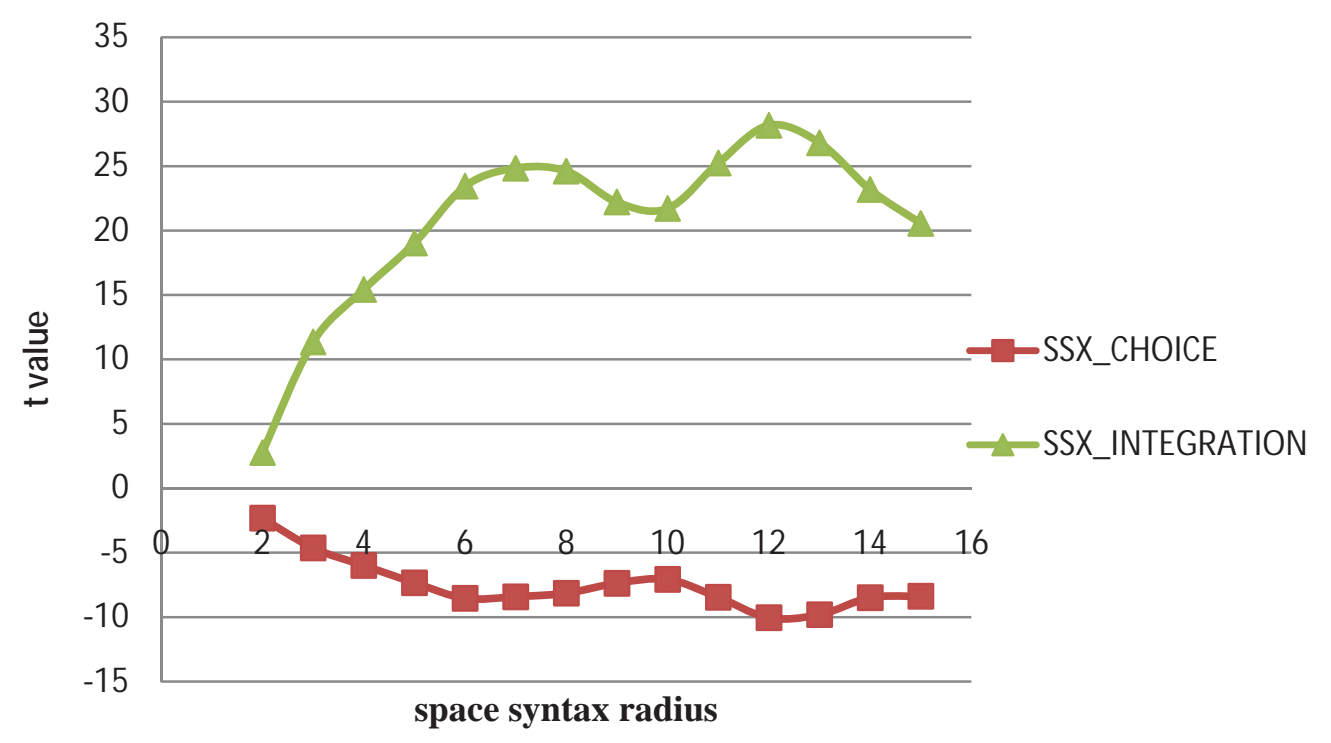

Figure 4.3Locational characteristics t value change for model I (c)

Source: author

\subsubsection{Aggregate data}

In order to compare the efficacy of network accessibility measures in hedonic models at different levels of aggregation I repeat the analysis already done using house prices aggregated to Census output areas. For this dataset, there are 322 observations of which only 317 are of use, as there are 5 output areas with missing values of floor area. The descriptive statistics for the hedonic variables are listed in Table 4.8, showing that $2.77 \%$ of the buildings are new buildings, while $82.2 \%$ properties are freehold. Terraced houses makeup the largest portion of buildings (50.7\%), and flats, the second largest portion. Only $3.83 \%$ of the output areas are classified as 'constrained by circumstances'; in contrast, most of the people (23.29\%) are classified as 'living in cities'. All these data are transformed from the disaggregated data, calculating the mean within each output area.

Table 4.8Descriptive Statistics for aggregated dataset 


\begin{tabular}{|c|c|c|c|c|c|}
\hline & $\mathrm{N}$ & Minimum & Maximum & Mean & Std. Deviation \\
\hline Ave_LN_PRICE & 322 & 10.690 & 12.849 & 11.770 & 0.403 \\
\hline Ave_LN_FL & 317 & 2.023 & 8.975 & 4.662 & 0.689 \\
\hline Ave_LN_D & 322 & 12.806 & 12.820 & 12.811 & 0.004 \\
\hline Ave_LN_D1 & 322 & 12.806 & 12.820 & 12.811 & 0.004 \\
\hline Ave_LN_D2 & 322 & 12.806 & 12.820 & 12.811 & 0.004 \\
\hline Ave_LN_D3 & 322 & 12.806 & 12.820 & 12.811 & 0.004 \\
\hline Ave_LN_D4 & 322 & 12.806 & 12.820 & 12.811 & 0.004 \\
\hline Ave_DU_N & 322 & 0.000 & 0.714 & 0.028 & 0.094 \\
\hline Ave_DU_D & 322 & 0.000 & 0.977 & 0.112 & 0.190 \\
\hline Ave_DU_SE & 322 & 0.000 & 1.000 & 0.254 & 0.239 \\
\hline Ave_DU_T & 322 & 0.000 & 1.000 & 0.508 & 0.333 \\
\hline Ave_DU_F & 322 & 0.000 & 1.000 & 0.126 & 0.198 \\
\hline Ave_DU_TE & 322 & 0.000 & 1.000 & 0.822 & 0.225 \\
\hline Ave_DU_BC & 322 & 0.000 & 1.000 & 0.152 & 0.360 \\
\hline Ave_DU_CL & 322 & 0.000 & 1.000 & 0.233 & 0.423 \\
\hline Ave_DU_PS & 322 & 0.000 & 1.000 & 0.189 & 0.392 \\
\hline Ave_DU_CC & 322 & 0.000 & 1.000 & 0.084 & 0.278 \\
\hline Ave_DU_TT & 322 & 0.000 & 1.000 & 0.208 & 0.407 \\
\hline Ave_DU_MU & 322 & 0.000 & 1.000 & 0.134 & 0.341 \\
\hline Ave_Y2000 & 322 & 0.000 & 0.448 & 0.115 & 0.062 \\
\hline Ave_Y2001 & 322 & 0.000 & 0.500 & 0.141 & 0.072 \\
\hline Ave_Y2002 & 322 & 0.000 & 0.500 & 0.146 & 0.066 \\
\hline Ave_Y2003 & 322 & 0.000 & 0.500 & 0.126 & 0.059 \\
\hline Ave_Y2004 & 322 & 0.000 & 0.667 & 0.121 & 0.068 \\
\hline Ave_Y2005 & 322 & 0.000 & 0.429 & 0.096 & 0.056 \\
\hline Ave_Y2006 & 322 & 0.000 & 0.389 & 0.124 & 0.059 \\
\hline Ave_Y2007 & 322 & 0.000 & 0.443 & 0.119 & 0.060 \\
\hline Ave_Y2008 & 322 & 0.000 & 0.333 & 0.012 & 0.025 \\
\hline Ave_CH_400M & 322 & 0.000 & 2.887 & 1.433 & 0.599 \\
\hline Ave_INT_400M & 322 & 8.135 & 67.355 & 27.958 & 13.315 \\
\hline Ave_CH_800M & 322 & 0.000 & 3.748 & 2.096 & 0.803 \\
\hline Ave_INT_800M & 322 & 10.368 & 167.199 & 65.275 & 35.394 \\
\hline Ave_CH_1200M & 322 & 0.000 & 4.260 & 2.447 & 0.925 \\
\hline Ave_INT_1200M & 322 & 16.185 & 265.339 & 113.913 & 61.073 \\
\hline Ave_CH_1600M & 322 & 0.000 & 4.687 & 2.676 & 1.004 \\
\hline Ave_INT_1600M & 322 & 24.794 & 385.980 & 171.363 & 89.420 \\
\hline Ave_CH_2000M & 322 & 0.000 & 5.008 & 2.839 & 1.055 \\
\hline Ave_INT_2000M & 322 & 38.914 & 509.813 & 231.581 & 115.489 \\
\hline Ave_CH_2500M & 322 & 0.000 & 5.293 & 2.993 & 1.110 \\
\hline Ave_INT_2500M & 322 & 65.608 & 620.645 & 308.213 & 142.766 \\
\hline Ave_CH_3000M & 322 & 0.000 & 5.476 & 3.118 & 1.154 \\
\hline Ave_INT_3000M & 322 & 91.846 & 726.716 & 388.102 & 167.112 \\
\hline Ave_CH_4000M & 322 & 0.000 & 5.750 & 3.310 & 1.220 \\
\hline
\end{tabular}




\begin{tabular}{lrrrrr} 
Ave_INT_4000M & 322 & 149.044 & 947.176 & 560.940 & 206.337 \\
Ave_CH_5000M & 322 & 0.000 & 6.027 & 3.449 & 1.270 \\
Ave_INT_5000M & 322 & 220.234 & 1221.000 & 738.890 & 241.592 \\
Ave_CH_6000M & 322 & 0.000 & 6.231 & 3.553 & 1.304 \\
Ave_INT_6000M & 322 & 305.793 & 1469.700 & 916.842 & 268.287 \\
Ave_CH_7000M & 322 & 0.000 & 6.400 & 3.635 & 1.326 \\
Ave_INT_7000M & 322 & 399.666 & 1695.000 & 1082.343 & 283.781 \\
Ave_CH_8000M & 322 & 0.000 & 6.537 & 3.692 & 1.348 \\
Ave_INT_8000M & 322 & 512.254 & 1837.800 & 1216.196 & 290.642 \\
Ave_CH_10000M & 322 & 0.000 & 6.692 & 3.746 & 1.367 \\
Ave_INT_10000M & 322 & 705.762 & 1938.300 & 1386.863 & 271.474 \\
Ave_LOG_CH & 322 & 0.000 & 6.736 & 3.743 & 1.377 \\
Ave_INT & 322 & 988.782 & 2018.600 & 1505.255 & 214.458 \\
Valid & 317 & & & & \\
\hline
\end{tabular}

Model II (a) uses the traditional hedonic variables including structure, locational, neighborhood effect, environmental and year attributes (Table 4.9 - shows the coefficients). The adjusted R-square is 0.770 ; log likelihood is 83.783; Akaie info criterion is -0.390 ; F-Test is 51.296 ; and the probability of the F-test is $0.000 .76 .97 \%$ of variation is explained by this model. Compared to the disaggregated data model, the aggregated data has better explanatory power due to less noise. In model II (a), "new building", "flat", "blue collar communities" and "constrained by circumstances" categories are insignificant at 5\% confidence interval. The reasons for this, is that they make up only a small portion of the variation in the output area compared with other attributes, and when aggregated this information is lost. Of the "year" variables, only 2006 and 2007 pass the P-value test, reflecting the peaking of property values in these years. VIFs are within the desired range indicating no multicolinearity. The analysis being based on output area, I expect there to be little or no heteroscedasticity. The White test of model II(a)(Table 4.10) confirms this with $\mathrm{N}^{*}$ adjust R-square being smaller than Chi-square(21). Moran's I test of model II(a)(Table 4.11) is 0.267 with a Z-score of 17.669 ( $\mathrm{P}=0.000)$, capturing the same evidence of spatial clustering as in the disaggregated model: high value output area cluster and a low value output area cluster. 
Model II(b) includes all 28 space syntax spatial accessibility variables averaged to output area (Table 4.9). The adjusted R-square is 0.836 and the log likelihood is 153.131. The Akaike info criterion is -0.657 and the F-value is 34.643 . The adjusted $R$ square has increased by approximately $8.65 \%$ by including the network accessibility measures and the log likelihood by $82.77 \%$. Additionally, the Akaike info criterion rose by $68.54 \%$ and the sum of squared residuals has been decreased by $35.47 \%$. The same improvements over the model without network accessibility measures is found, therefore, as in the disaggregated test. Of the locational variables "Distance to the CBD" drops out in model II(b), its explanatory power replaced by the space syntax spatial accessibility indices. The years "Y2006" and "Y2007" are still significant, but "Y2005" also become significant in model II (b). VIF scores for all variables increase dramatically compared to model II (a), with VIF scores for some of the structure variables beyond the safe limit. From this, I can infer that the structure information and traditional spatial information are highly correlated with the network configuration information at output area level. As can be seen from the table 4.9, most space syntax spatial accessibilities are insignificant at a 5\% confidence interval. Only a few integration values and one choice value pass the p-value. However, the VIF scores for most of the space syntax measures are over 10, which indicates strong multicoliniarity in this model. The $\mathrm{T}$ value is not reliable, but from the other performance indices like the adjusted R-square, log likelihood and AIC, I may still conclude that at the output area level the urban network structure has a significant affect on housing price, especially at a small scale where traditional location information such as distance from CBD is less valid. The White test for model II (b) (Table 4.10) shows that $\mathrm{N}^{*}$ adjust R-square < Chi-square(48), which indicates that there is no evidence of heteroscedasticity in the residuals, the same result as model II(a). So this model has strong predictive power. In table 4.11, Moran's I index is 0.097, with a Z-score of $6.581(\mathrm{p}=0.000)$, the same conclusion as model II (a), but with an improvement in Z-score of $62.75 \%$, which confirms again that explanatory 
power of the space syntax urban configuration information using aggregate data.

Table 4.9Regression results of Model II (a) and (b)

\begin{tabular}{|c|c|c|c|c|c|c|c|c|c|c|}
\hline & \multicolumn{5}{|c|}{ Model II(a) } & \multicolumn{5}{|c|}{ Model II(b) } \\
\hline & \multirow{2}{*}{\multicolumn{3}{|c|}{$\begin{array}{l}\text { Adjusted R-squared } \\
\text { Akaike info criterion }\end{array}$}} & \multicolumn{2}{|l|}{0.770} & \multicolumn{3}{|c|}{ Adjusted R-squared } & \multicolumn{2}{|c|}{0.837} \\
\hline & & & & -0.390 & & & Akaike info & criterion & -0.661 & \\
\hline & \multicolumn{3}{|c|}{ Log likelihood } & 83.782 & & & Log likelihood & & 153.8 & \\
\hline & \multicolumn{2}{|c|}{ F-statistic } & \multicolumn{3}{|c|}{51.296} & \multicolumn{3}{|c|}{ F-statistic } & \multicolumn{2}{|c|}{34.827} \\
\hline & Coeff. & SD. Coeff. & $\mathrm{t}$ & Sig. & VIF & Coeff. & SD. Coeff. & $\mathrm{t}$ & Sig. & VIF \\
\hline Constant & 563.157 & & 9.096 & 0.000 & & -14.464 & 117.844 & -0.123 & 0.902 & \\
\hline Ave_LN_FL & 0.096 & 0.165 & 4.372 & 0.000 & 1.957 & 0.061 & 0.021 & 2.892 & 0.004 & 2.573 \\
\hline Ave_LN_D & -43.129 & -0.379 & -8.929 & 0.000 & 2.479 & 1.972 & 9.198 & 0.214 & 0.830 & 12.685 \\
\hline Ave_DU_N & -0.133 & -0.030 & -0.919 & 0.359 & 1.509 & -0.100 & 0.135 & -0.736 & 0.462 & 1.856 \\
\hline Ave_DU_D & 1.341 & 0.617 & 14.683 & 0.000 & 2.424 & 1.431 & 0.087 & 16.516 & 0.000 & 3.079 \\
\hline Ave_DU_SE & 0.527 & 0.313 & 8.068 & 0.000 & 2.072 & 0.523 & 0.065 & 7.997 & 0.000 & 2.939 \\
\hline Ave_DU_F & -0.061 & -0.030 & -0.412 & 0.681 & 7.303 & -0.287 & 0.146 & -1.961 & 0.051 & 10.177 \\
\hline Ave_DU_TE & 0.177 & 0.100 & 1.518 & 0.130 & 5.932 & -0.101 & 0.117 & -0.859 & 0.391 & 8.443 \\
\hline Ave_DU_BC & -0.027 & -0.024 & -0.562 & 0.574 & 2.555 & 0.012 & 0.049 & 0.247 & 0.805 & 3.658 \\
\hline Ave_DU_CL & 0.217 & 0.229 & 5.499 & 0.000 & 2.382 & 0.124 & 0.038 & 3.252 & 0.001 & 3.166 \\
\hline Ave_DU_PS & 0.162 & 0.158 & 2.832 & 0.005 & 4.278 & 0.122 & 0.054 & 2.270 & 0.024 & 5.316 \\
\hline Ave_DU_CC & -0.002 & -0.001 & -0.036 & 0.971 & 1.852 & 0.009 & 0.050 & 0.180 & 0.857 & 2.313 \\
\hline Ave_DU_TT & 0.203 & 0.206 & 5.103 & 0.000 & 2.237 & 0.138 & 0.039 & 3.576 & 0.000 & 2.972 \\
\hline Ave_Y2001 & -0.422 & -0.072 & -1.746 & 0.082 & 2.318 & -0.338 & 0.465 & -0.726 & 0.468 & 9.872 \\
\hline Ave_Y2002 & -0.395 & -0.063 & -1.587 & 0.114 & 2.153 & -0.560 & 0.434 & -1.289 & 0.198 & 10.579 \\
\hline Ave_Y2003 & -0.054 & -0.007 & -0.207 & 0.836 & 1.715 & -0.307 & 0.457 & -0.672 & 0.502 & 10.273 \\
\hline Ave_Y2004 & 0.427 & 0.064 & 1.593 & 0.112 & 2.240 & 0.071 & 0.448 & 0.159 & 0.874 & 7.228 \\
\hline Ave_Y2005 & 0.525 & 0.073 & 2.070 & 0.039 & 1.710 & 0.247 & 0.418 & 0.590 & 0.556 & 7.718 \\
\hline Ave_Y2006 & 0.724 & 0.105 & 2.812 & 0.005 & 1.933 & 0.393 & 0.441 & 0.890 & 0.374 & 7.296 \\
\hline Ave_Y2007 & 0.754 & 0.105 & 2.890 & 0.004 & 1.812 & 0.425 & 0.433 & 0.982 & 0.327 & 7.734 \\
\hline Ave_Y2008 & 0.483 & 0.030 & 0.927 & 0.354 & 1.464 & 0.455 & 0.444 & 1.025 & 0.306 & 7.407 \\
\hline Ave_CH_400M & & & & & & -0.077 & 0.069 & -1.120 & 0.264 & 19.903 \\
\hline Ave_INT_400M & & & & & & -0.001 & 0.003 & -0.262 & 0.794 & 14.784 \\
\hline Ave_CH_800M & & & & & & 0.090 & 0.205 & 0.440 & 0.660 & 312.292 \\
\hline Ave_INT_800M & & & & & & -0.002 & 0.002 & -0.849 & 0.397 & 78.313 \\
\hline Ave_CH_1200M & & & & & & 0.025 & 0.342 & 0.074 & 0.941 & 1151.356 \\
\hline Ave_INT_1200M & & & & & & 0.000 & 0.002 & 0.220 & 0.826 & 189.263 \\
\hline Ave_CH_1600M & & & & & & 0.256 & 0.416 & 0.615 & 0.539 & 1997.030 \\
\hline Ave_INT_1600M & & & & & & -0.001 & 0.002 & -0.380 & 0.704 & 433.693 \\
\hline Ave_CH_2000M & & & & & & -0.220 & 0.420 & -0.523 & 0.601 & 2247.920 \\
\hline
\end{tabular}




\begin{tabular}{llllrr}
\hline Ave_INT_2000M & & & & & \\
Ave_CH_2500M & 0.004 & 0.002 & 2.130 & 0.034 & 500.326 \\
Ave_INT_2500M & -1.246 & 0.517 & -2.408 & 0.017 & 3766.111 \\
Ave_C & -0.002 & 0.002 & -1.122 & 0.263 & 572.672 \\
Ave_CH_3KM & 1.056 & 0.473 & 2.233 & 0.026 & 3399.330 \\
Ave_INT_3KM & 0.001 & 0.001 & 1.109 & 0.268 & 430.183 \\
Ave_CH_4KM & 1.031 & 0.492 & 2.094 & 0.037 & 4113.178 \\
Ave_INT_4KM & -0.002 & 0.001 & -2.280 & 0.023 & 315.126 \\
Ave_CH_5KM & -1.140 & 0.561 & -2.031 & 0.043 & 5799.182 \\
Ave_INT_5KM & -0.001 & 0.001 & -1.698 & 0.091 & 398.874 \\
Ave_CH_6KM & 0.198 & 0.389 & 0.509 & 0.611 & 2931.524 \\
Ave_INT_6KM & 0.003 & 0.001 & 3.119 & 0.002 & 572.207 \\
Ave_CH_7KM & 0.189 & 0.517 & 0.366 & 0.715 & 5362.242 \\
Ave_INT_7KM & -0.001 & 0.001 & -1.746 & 0.082 & 637.047 \\
Ave_CH_8KM & 0.150 & 0.498 & 0.301 & 0.763 & 5131.302 \\
Ave_INT_8KM & 0.001 & 0.001 & 1.127 & 0.261 & 386.288 \\
Ave_CH_10KM & -0.217 & 0.321 & -0.675 & 0.500 & 2196.124 \\
Ave_INT_10KM & 0.002 & 0.000 & 3.404 & 0.001 & 180.997 \\
Ave_LOG_CH & -0.096 & 0.110 & -0.868 & 0.386 & 262.273 \\
Ave_INT & -0.001 & 0.000 & -3.516 & 0.001 & 83.695 \\
\hline
\end{tabular}

Table 4.10White test for Model II (a) and (b)

\begin{tabular}{|c|c|c|c|}
\hline \multicolumn{2}{|c|}{ Model II (a) } & \multicolumn{2}{|c|}{ Model II (b) } \\
\hline $\mathrm{N}=317$ & Adjust R-square 0.077416 & $\mathrm{~N}=317$ & Adjust R-square 0.031295 \\
\hline $\mathrm{N} *$ Adjust R-Square $=24.541$ & Chi-square $(21)=32.671$ & $\mathrm{~N}^{*}$ Adjust R-Square $=9.921$ & Chi-square $(48)=65.171$ \\
\hline \multicolumn{2}{|c|}{$\mathrm{N}^{*}$ Adjust R-Square $<$ Chi-square(21) } & \multicolumn{2}{|c|}{$\mathrm{N}^{*}$ Adjust R-Square $<$ Chi-square(48) } \\
\hline
\end{tabular}

Table 4.11Global Moran's I for Model II (a) and (b)

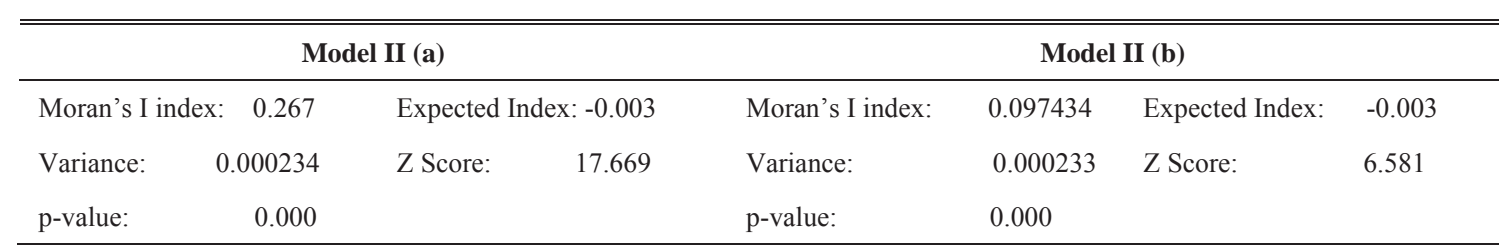

Finally, I repeat the sensitivity analysis of models to the spatial scale of network connectivity analysis. 14 versions of model II(c) are computed (Table 4.12). The tables illustrate the comparison of each model's T-value and P-value of choice and 
integration. In all 14 models, "DU_D”, "DU_F”, “DU_TE”, "DU_BC”, “DU_CC" categories are insignificant. Most 'year' dummy variables are invalid in the models except "Y2006" and "Y2007". "Distance to CBD" has a consistent negative impact on housing price which is as expected. VIF for each model is within the desired range. Not all choice and integration variables are significant at the aggregated level. All choice values are insignificant, suggesting that flows through the urban road layout have no impact on the house price at the output area level. On the other hand, integration value remains a significant determinant with aggregate data. These results reflect the street network characteristics of Cardiff, as a variety of street segments are dead end. If I assume that many dead end streets intersect with one main street, the integration value of the dead end road is similar to the high value of the main street, as the segment still has easy access to many other segments. For this reason, I can conclude that high integration values normally cluster. In contrast, while choice value of a main street will be high, with lots traffic movement, the choice value of a dead end road, even if links to a main road, is very low as it is a dead end and has less movement through it. There is likely to be a big stand deviation of choice value in each output area. Hence, when aggregating to the output area, choice value characteristics will be lost. If I are correct in associating integration with positive externalities and choice with negative at the disaggregate level, this may indicate that aggregation somehow smoothes or conceals the effects of negative externalities but not accessibility benefits.

Surprisingly in figure 3.6, integration at radius $2500 \mathrm{~m}$ gives the highest explanation $(81.2 \%)$ rather than the $7000 \mathrm{~m}$ radius in model I (c). The T-values for integration rise steadily from 3.381 peaking at 8.028 at a radius of $2500 \mathrm{~m}$ and then decline slowly as the radius increases. This is because when aggregating the data, I calculated the mean for all attributes within the output area, a process that ignores the variance of housing prices and thus loses information. It is likely that in the aggregated model, the optimal scale of measuring accessibility is constrained by the data structure and it does not so 
well indicate people's behavior (which is better captured in the disaggregated model where more information is retained).

Table 4.12 Model II (c): Different Radii - T value comparisons

\begin{tabular}{|c|c|c|c|c|c|c|c|c|c|c|}
\hline & \multicolumn{2}{|c|}{$400 \mathrm{M}$} & \multicolumn{2}{|c|}{$800 \mathrm{M}$} & \multicolumn{2}{|c|}{$1200 \mathrm{M}$} & \multicolumn{2}{|c|}{$1600 \mathrm{M}$} & \multicolumn{2}{|c|}{$2000 \mathrm{M}$} \\
\hline & $\mathrm{CH}$ & INT & $\mathrm{CH}$ & INT & $\mathrm{CH}$ & INT & $\mathrm{CH}$ & INT & $\mathrm{CH}$ & INT \\
\hline $\mathrm{t}$ & -1.84 & 3.381 & -0.054 & 5.188 & -1.59 & 6.332 & -1.712 & 7.124 & -1.611 & 7.913 \\
\hline Sig. & 0.067 & 0.001 & 0.142 & 0 & 0.113 & 0 & 0.088 & 0 & 0.108 & 0 \\
\hline \multirow[t]{3}{*}{ Ad- $R^{2}$} & \multicolumn{2}{|c|}{0.778} & \multicolumn{2}{|c|}{0.79} & \multicolumn{2}{|c|}{0.799} & \multicolumn{2}{|c|}{0.805} & \multicolumn{2}{|c|}{0.811} \\
\hline & \multicolumn{2}{|c|}{$2500 \mathrm{M}$} & \multicolumn{2}{|c|}{$3000 \mathrm{M}$} & \multicolumn{2}{|c|}{$4000 \mathrm{M}$} & \multicolumn{2}{|c|}{$5000 \mathrm{M}$} & \multicolumn{2}{|c|}{$6000 \mathrm{M}$} \\
\hline & $\mathrm{CH}$ & INT & $\mathrm{CH}$ & INT & $\mathrm{CH}$ & INT & $\mathrm{CH}$ & INT & $\mathrm{CH}$ & INT \\
\hline $\mathrm{t}$ & -1.401 & 8.028 & -1.262 & 7.785 & -0.874 & 6.406 & -0.787 & 6.225 & -0.983 & 7.154 \\
\hline Sig. & 0.162 & 0 & 0.208 & 0 & 0.383 & 0 & 0.432 & 0 & 0.326 & 0 \\
\hline \multirow[t]{3}{*}{ Ad- $R^{2}$} & \multicolumn{2}{|c|}{0.812} & \multicolumn{2}{|c|}{0.81} & \multicolumn{2}{|c|}{0.799} & \multicolumn{2}{|c|}{0.798} & \multicolumn{2}{|c|}{0.805} \\
\hline & \multicolumn{2}{|c|}{$7000 \mathrm{M}$} & \multicolumn{2}{|c|}{$8000 \mathrm{M}$} & \multicolumn{2}{|c|}{$10000 \mathrm{M}$} & \multicolumn{2}{|c|}{ Infinite } & & \\
\hline & $\mathrm{CH}$ & INT & $\mathrm{CH}$ & INT & $\mathrm{CH}$ & INT & $\mathrm{CH}$ & INT & & \\
\hline $\mathrm{t}$ & -1.138 & 7.639 & -1.179 & 7.647 & -0.957 & 7.048 & -1.134 & 6.423 & & \\
\hline Sig. & 0.256 & 0 & 0.239 & 0 & 0.34 & 0 & 0.258 & 0 & & \\
\hline Ad- $R^{2}$ & \multicolumn{2}{|c|}{0.809} & \multicolumn{2}{|c|}{0.809} & \multicolumn{2}{|c|}{0.804} & \multicolumn{2}{|c|}{0.799} & & \\
\hline
\end{tabular}

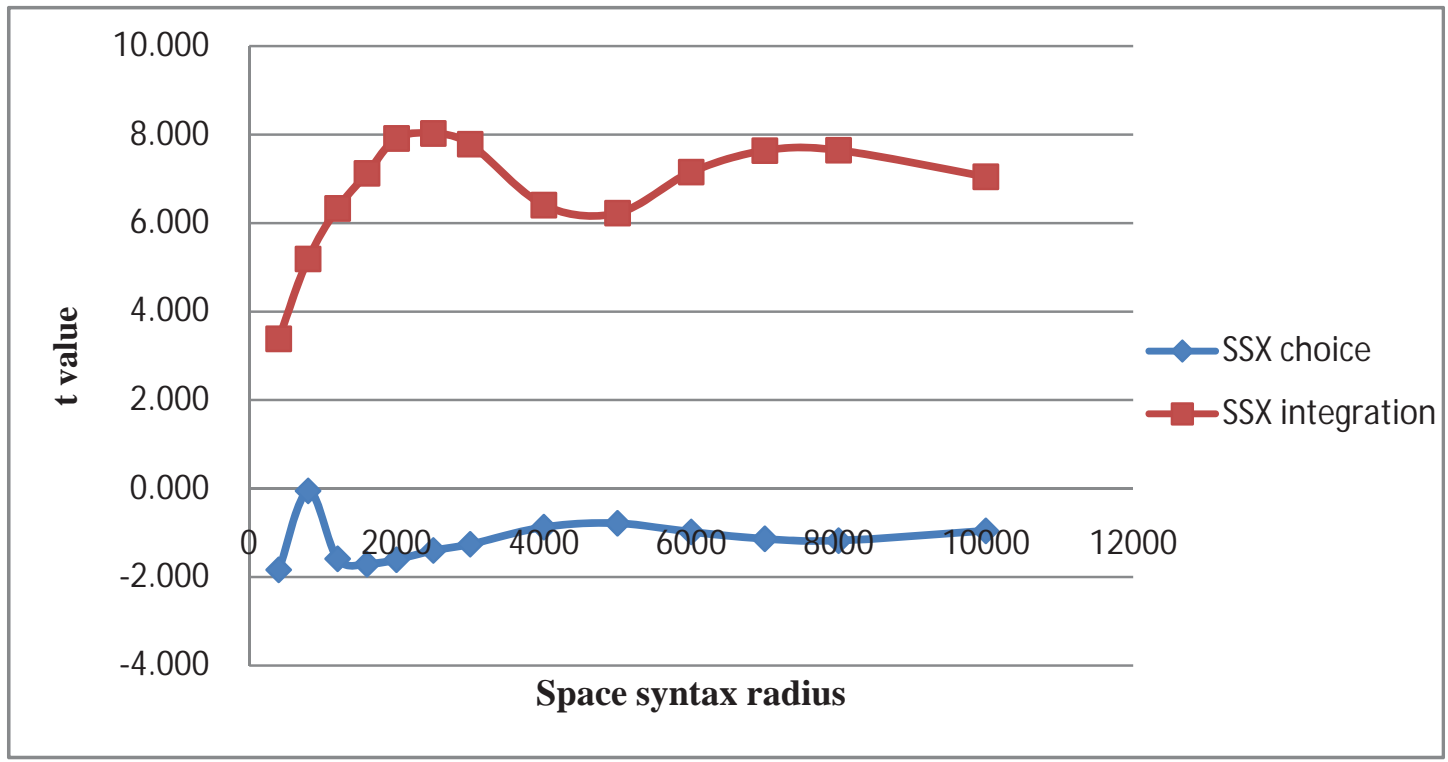

Figure 4.4Locational characteristics t value change for model II (c)

Source: author 


\subsubsection{Discussion of disaggregated data and aggregated data}

Comparing the space syntax spatial accessibility performance in disaggregated and aggregated data, I conclude that the estimation of models improved, the adjust R-square increased and the sum of squared residuals reduced; all of which tell us that urban spatial structure has a significant and important impact on house price. Furthermore, there is no doubt that network accessibility measures can capture additional information about the uniqueness of locations, which reduces the problem of spatial autocorrelation. Although spatial autocorrelation has not been removed completely in our models, I have nevertheless confirmed that if I have more detailed information of the characteristics of different of spaces, I can reduce the spatial autocorrelation error in models. I would therefore expect that if I can produce additional spatial configuration information, as with our network models, Moran's I will be even smaller. Whether in disaggregated or aggregated data, integration accessibility always has a positive impact on the property value, which indicates that if a place has high connectivity it will command a higher value for residential use.

Aggregated data gives better estimation: nearly $83.6 \%$ of observed variation is explained by model II(b). The aggregated data has no heteroscedasticity in the residuals, which means better ability for prediction. Another difference is that model II (b) shows that space syntax variables employed in aggregated data gives a better result for reducing the spatial effect. Finally, in disaggregated data, choice value has a negative impact on housing price, indicating that locations with configurationally less likelihood of congestion have higher prices. The optimal scale of accessibility generated from the disaggregated model can be taken as an indication of the scale at which peoples' movements through the street network impact housing price. In the aggregated model, this information seems to be lost.

Generally, analysis based on aggregated data is more valuable for informing policy, 
especially where units of aggregation are political entities or can be mapped on to the same. With aggregated data, it is possible, for example, to experiment with alternative infrastructure improvement projects and observe the effect on the mean space syntax spatial accessibility. Alternatively, I can estimate whether a particular project will increase land values through its influence on urban configuration.

\subsection{Summary}

The principal objective of this study was to test whether urban configuration can be shown to affect house prices. I find that the addition of configurational accessibility indicators measures on a network model raise the \% variation explained in a hedonic model of Cardiff house prices by $6.4 \%$.

I also wanted to know how an aggregate model with network accessibility variables compared to a disaggregate model; what the optimal radius is for measuring network effects on house price; and what additional advantages network measures have compared to conventional Euclidean measures in respect of improving model specification. In these regards, I found the following (Table, 4.13).

Table 4.13Comparison the results with previous studies

\begin{tabular}{|c|c|c|c|c|c|}
\hline & Data type & $\begin{array}{c}\text { SSX } \\
\text { measurement }\end{array}$ & SSX index & $\begin{array}{l}\text { Sig } \\
\mathrm{n}\end{array}$ & Econometrics results \\
\hline $\begin{array}{l}\text { Matthews and Turnbull, } \\
\qquad 2007\end{array}$ & $\begin{array}{l}\text { Disaggregat } \\
\text { ed }\end{array}$ & Typology & $\begin{array}{l}\text { Integration } \\
\text { Segments } \\
\text { ratio }\end{array}$ & $\begin{array}{l}+/- \\
+/-\end{array}$ & $\begin{array}{l}\text { Significant within } 1400 \text { feet walking } \\
\text { distance } \\
\text { Significant within } 1400 \text { feet walking } \\
\text { distance }\end{array}$ \\
\hline $\begin{array}{l}\text { Enstrom and Netzell, } \\
\qquad 2008\end{array}$ & $\begin{array}{l}\text { Disaggregat } \\
\text { ed }\end{array}$ & Typology & Integration & + & Significant \\
\hline \multirow{3}{*}{ This study } & Disaggregat & & Integration & + & $\begin{array}{l}\text { Significant all radii, largest t value at } 7 \mathrm{~km} \\
\text { radii }\end{array}$ \\
\hline & ed & Segments radii & Choice & - & $\begin{array}{l}\text { Significant all radii, smallest } \mathrm{t} \text { value at } \\
7 \mathrm{~km} \text { radii }\end{array}$ \\
\hline & Aggregated & & Integration & + & Significant all radii, largest $t$ value at \\
\hline
\end{tabular}


Diagnostic tests indicate that network accessibility measures can improve the robustness of hedonic models, in particular reducing the problem of autocorrelation. Urban network configuration has a significant impact on house price at both aggregated and disaggregated levels of analysis.Integration and choice both significantly affect house price for disaggregated data; integration value also has a positive impact on house price whereas choice value has a negative impact. I hypothesise that one captures positive grid network externality effects and the other negative and offer this as a testable proposition to be investigated in subsequent research. With aggregated data, integration remains significantly positively correlated with house price, whilst choice value becomes insignificant. I hypothesise that this is due to the particular style of street network in the case study city of Cardiff, with many dead-end streets linked directly to main streets, meaning that there is a higher variance for choice, which is lost at the aggregate level. Finally, our experiment shows that detailed urban configuration indicators measured from a network model of the urban grid had a stronger power in explaining variance in house prices than the traditional measure of distance to $\mathrm{CBD}$ and without the scale limitation of that measure. 


\section{Chapter Five:}

\section{Identification of housing submarkets by urban configurational features}

\subsection{Introduction}

Rosen (1974) introduced the idea of the hedonic price model where in an equilibrium market with complete competition, the marginal implicit price estimated for the characteristics of property is equal to marginal people's willingness to pay for these housing attributes. However, the housing market is not a uniform entity, rather it could be considered as distinctive segments across space, and the segmentation of demand and supply between geographical could lead to the spatial disequilibrium. Although there is general agreement amongst researchers that housing submarkets exists, there is no single and coherent definition of a housing submarket (Adair et al. 1996; Watkins 2001).

Commonly, there are two categories of specifications for housing submarkets: spatial and non-spatial methods. Spatial specifications emphasise a pre-defined geographic area with people's homogenous choice preferences as the main index (e.g. inner/outer city, north/south, political districts, and postcode districts) (Gabriel and Wolch 1984b; Maclennan et al. 1987a; Hancock 1991). When building type is diverse and its spatial segregation distinct, the housing submarket can also be specified by building structure types (e.g.,detached, semidetached, terrace house and flat) and/or structure characteristics (e.g., property age, numbers of room)(Dale-Johnson 1982; Bajic 1985; Allen et al. 1995; Adair et al. 1996). In addition, some researchers have found a specification with nested spatial and building structure characteristics has better performance (Goodman 1981; Maclennan and Tu 1996; Bourassa et al. 1999). 
By contrast, non-spatial sub-market specifications emphasise accuracy of estimation, advocating a data driven approach, without a pre-defined geographic area. These specifications rely on statistical hierarchical clustering techniques to explore patterns in people's homogeneous demand (e.g., income, socioeconomic status, household mobility) (Bryk and Lee 1992; Feitelson 1993; Goodman and Thibodeau 1998; Jones et al. 2004; Alkay 2008). However, these specification methods are criticized for being unstable over time (e.g. Bourassa et al. 1999).

Despite these definitional uncertainties specifications for housing submarket are widely accepted both in academic and practical fields in most developed countries with mature urban land markets. There is less knowledge about how to delineate sub markets in property markets where the building type is simplex (apartments) and social neighbourhood characteristics are not long established and changing quickly over time, such as most cities in China. Due to therapid rate of urbanization and importance placed by the national government on protecting land for food supply, high-density development is the most common solution to fight population expansion in China and many other developing counties. One consequence is simplex building types (high-rise flat). This means that the physical attributes of housing is not so singularly important in determining or delimiting housing sub-markets as it is in western countries where cities grew much more slowly. In addition, large scale and rapid rural migration in China and other developing countries tends to break the original social spatial structure within geographic areas, and mean that neighbourhood characteristics change constantly.

This study attempts to specify submarkets by urban configurational features at the street-level, using a case study of Cardiff, UK. It is hypothesized that accessibility is jointly purchased with residential location (e.g. Michelson 1977). Different levels and types of accessibility are acquired by the neighborhood choice of residents(Handy and 
Niemeier 1997).Cardiff was chosen as a laboratory for the basic scientific experiment because of good quality and readily available data. Chapter Six goes on to extend the concept to China to look at the power of network configuration to predict housing market change in the high density simplex-dominated city of Nanjing.

The objectives of this the study reported in this chapter are two folds: firstly, to examine whether accessibility contained in urban configurational data measured from a network model of streets is associated with social neighborhood preferences and thus reflected in housing price. Secondly, the chapter compares the estimation results of the configurationally-specified housing submarkets with traditional spatial specifications. The research framework is composed of the following steps: firstly, the location-based social network analysis method known as space syntax will be applied in this study, for measuring accessibility in urban configuration using two indices "integration", and "choice" (defined in Chapter Three).Then, two new alternative specifications will be made for housing submarkets. One is based on the optimal space syntax accessibility radii, which will be chosen by the hedonic price model. The second one is based on the optimal space syntax accessibility radii plus building types. Next, a two-step clustering analysis will be applied for identifying the optimal numbers of clusters for housing submarket. The remaining steps will follow Schnare and Struyk (1976)'s approach to test the existence of housing submarkets and find the optimal number. Finally, the results of the traditional and of the network-informed approaches will be compared.

The structure of this study will be organized as follows: section two will review existing studies that explore specifications for identifying housing submarkets. In section three, the methodologies of space syntax network analysis, the specification of hedonic price model, two-step clustering and Chow test will be introduced. Section four introduces the study area and the dataset for this study in Cardiff, UK. The results are presented in section five, utilizing five different models (e.g. city-wide, 
building type, spatial joint building type, optimal space syntax accessibility, and the accessibility plus building type). Section six further discusses the implications of the empirical results. Finally, section seven summarizes the findings and concludes.

\subsection{Literature review}

\subsubsection{Specifications of housing submarket}

Housing submarkets are the cause of the housing markets' disequilibrium according to (Whitehead and Odling-Smee 1975). Straszheim (1975) first noted that the fundamental characteristics of the urban housing market are variation in housing characteristics and its price by location.

The definition of housing submarket seems difficult and not coherent. Maclennan et al (1987b)stated the housing market could be considered as "....... a simple theoretical construct with no specific form and often it has no qualitative, temporal or spatial dimensions". Rapkin (1953) definite housing submarket as “......every dwelling unit within a local housing market may be considered a substitute for every other unit".

Recently, that concept of housing submarket has been the key focus for some scholars. For example, Goodman and Thibodeau (1998) state that the cause of housing submarkets is due to people's demand for particular locations and/or particular types of housing at particular locations. A metropolitan housing area is always segmented into small submarkets due to either supply or demand-related factor (Goodman and Thibodeau 2003). Bau and Thibodeau (1998)note that housing submarkets are typically defined as geographic areas where the prices per unit of housing quantity (defined using some index of housing characteristics) are relatively constant.

Goodmanand Thibodeau(2007) highlight the importance of explicitly recognizing 
housing submarkets to housing market analysis: (1) it will improve the accuracy of estimations, (2) it can assist researchers to model properly in both spatial and temporal variation.(3) it can improve the lenders' and investors' abilities to price risk. Finally, housing submarket can reduce housing consumers' search and other transaction costs.

Given the importance of housing submarkets, many researchers have attempted to identify sources of spatial disequilibrium. There are two mainstream school of thoughts for identifying submarkets. The first one is spatial based and suggests that in geographic areas, current spatial divisions (e.g. census output areas) can also be used to define boundaries of the submarket with the price of housing (per unit of service) being assumed constant within. Early published studies about the specification of housing submarket are based on supply-side analysis, using pre-defined boundaries to identify areas of roughly homogenously priced housing, land and externality attributes using a specified geography. For example, Straszheim (1975) and Gabriel (1984) use the racial composition of districts; Sonsteilie and Portney(1980) use political districts; and Hancock (1991) uses postcode districts. Munro (1986) specified the Glasgow market with a pre-defined spatial boundary, by north and south of the river, and in inner and outer suburban areas. Michael and Smith (1990) attempted to segment the housing market by agent definition. In all these, there is an implicit assumption that willingness to pay for price per unit of housing characteristics is constant within the adopted geographic divisions.

Additionally, some researchers have acknowledged that building's structural characteristics reflect people's preferences from supply-side, with the willingness to pay for individual housing characteristics being constant within certain building categories. This had led to many scholars attempting to identify the housing submarket using building structural characteristics. For example, Dale-Johnson (1982) used factor analysis to group dwellings with similar characteristics in order to define 
housing submarkets. Allen et al. (1995) specified housing submarkets on the basis of dwelling types (e.g., condominiums, single-family homes, and apartments). Furthermore, some researchers note that when consumers consider purchasing different size and types of dwellings they will be constrained by their income or other household socioeconomic factors, and consequently they start to consider the demand-side characteristics rather than the characteristics of stock per se. For example, Greaves (1985) pointed out the existence of submarkets in which lower income purchasers are primarily interested in satisfying basic accommodation needs. This contrasts with higher income purchasers, who have a wider range of requirements governing their housing choice.

Approaches such as those mentioned above assume that people are willing to pay the same price per unit for a property regardless of it being situated in the city center or in the suburbs. However, the monocentric trade-off model of urban economics emphasizes the trade-off of transportation costs against land rents(Alonso 1964a; Muth 1969; Mills 1972).Therefore, many researchers have recognized the importance of nested spatial and building structural characteristics for housing submarkets specification, giving a more complete supply-side specification. For example, Bajic (1985) employed structure characteristics such as floor area and lot size and the distance to the CBD as a spatial component. Adair et al. (1996) attempted to subdivide the city into inner city, middle city and outer city, and identified nine submarkets for the Belfast housing market based on terraced, semidetached, and detached dwelling within each area. Watkins (2001) provides a detailed review of the alternative approaches that housing economists have employed for characterizing housing submarkets. Using transaction data for the Glasgow housing market, he examined three alternative approaches for delineating housing submarket: (1) spatially stratified housing submarkets, (2) submarkets based on the similarity of structure characteristics and (3) a hybrid definition that nests dwelling characteristics based submarket within a spatially defined submarket. He concluded that the nested model provided the best 
empirical approach for delineating submarkets. This is to be expected since segmentation and clustering of preferences can be expected to occur on all of the significant dimensions of housing choice.

However, spatial-based specifications have been criticized for their inaccuracy. Due to rapid urbanization and the emergence of polycentricity, the urban system has become more complex and consequently the social spatial structure is transforming and social spatial segregation has been enhanced, especially in mega cities, such as Los Angeles and Shanghai. The original spatial models used to characterize the spatial divisions of preferences appears in these types of cities to be less effective in reflecting people's preference and choice for each characteristic. The risk is that traditional approaches will underestimate of the amount of submarkets. Furthermore, the spatial-based specification often seems to bead hoc because of the use of pre-identified geographical boundaries. It also requires a prior acknowledge of the local context and therefore renders scientific study difficult and not easily replicated.

In contrast, the second main stream approach to specifying housing submarkets is non-spatial, and attempts to identify new non-spatial groups or clusters where people have similar consumer perceptions of the individual characteristics of housing. So the clustering is by non-spatial consumer preference dimensions rather than by geographic area and physical stock characteristics.

Statistical approaches used to define groups for housing submarket, have included principal component analysis, factor analysis and hierarchical analysis (Dale-Johnson 1982; Maclennan and Tu 1996; Goodman and Thibodeau 1998; Bourassa et al. 1999). Moreover, some scholars attempt to identify the housing submarket by more advanced statistical technique. For example, Kauko et al. (2002) applied neural network analysis whilst Meen and Meen(2003) used cellular automata and discrete choice models and Hwang and Thill (2009) chose to apply fuzzy clustering to define urban 
housing submarket.

Compared to the spatial-based specifications, the non-spatial based determinants rely more on statistical methods, and place more importance on the maximum accuracy of estimation; specifying groups by demand indicators; and data-driven multi-variate boundaries. For example, Maclennan and Tu (1996) use principle component analysis to identify housing submarkets in Glasgow. It was found that in cluster analysis, the $\mathrm{K}$ means algorithm is better than the Ward means. Bourassa et al. (1999) segmented the Sydney and Melbourne housing markets by applying principal components and cluster analysis to a variety of neighborhood attributes, spatial and structure characteristics, and lettings data in order to determine submarkets. Day (2003) used hierarchical clustering techniques to identify property submarket by attributes of dwelling types, locations and people's socioeconomic characteristics.

Bourassa et al. (2003) examined two submarket constructions: (1) geographically concentrated "sale areas" used by local real estate appraisers in New Zealand and (2) a spatial submarket construction obtained by applying cluster analysis to the most influential factors generated from property, neighborhood and location attributes. They compared hedonic housing price predictions generated from these alternatives to a single equation for the entire city. They concluded that while s statistically generated submarket significantly increased hedonic house price prediction accuracy relative to the single equation model, the statistically generated submarket did not outperform the sale area submarket model.

TU et al.(2007) first investigated housing market segmentation through housing price spatial autocorrelation. They sought to let the data define urban housing market segmentation, rather than use the traditional administrative or any pre-defined boundaries to specify submarkets. They thought that the structure of spatial autocorrelation in the residual should reflect the neighborhood structure where errors 
are more likely to be correlated within submarkets than across submarkets, and therefore that controlling for submarkets in hedonic equations will substantially reduce estimation errors. The empirical results showed that the identified housing submarket structure improved the standard error by $17.5 \%$.

While spatial methods have been criticized for being arbitrary, non-spatial specification methods have been criticized for being unstable over time (e.g. Bourassa et al. 1999). Jones et al.(2003) point out that specifying housing submarkets by neighborhood characteristics is unstable, as the market is constantly changing. The approach has also been criticized for its non-spatial nature and the difficulties it presents for policy interventions. Bourassa et al. (2003) stated that non-spatial approach for submarkets in the hedonic price model can not fully demonstrate the impact of spatial attributes on housing prices. Goodman and Thibodeau(2003; 2007) emphasize that researchers should impose submarket spatial boundaries rather than deriving them through modeling, as practitioners and policymakers need a clear grasp of the housing submarket structure system across space (Jones et al. 2003). Some scholars even state that spatial characteristics are more important in the specification of submarkets (Palm 1978; Goodman 1981; Michaels and Smith 1990), even some evidence suggest that geographical submarkets are more meaningful, for policy implication, rather than improving prediction accuracy (Bourassa et al. 2003; Goodman and Thibodeau 2003; Goodman and Thibodeau 2007). Arguments behind such a view rest among other things on the importance of neighborhood labeling and the resilience of labels of time.

\subsubsection{Accessibility and social neighborhood characteristics}

There is a rich literature, which argues that accessibility is the most essential determinant of property value (e.g. Heikkila et al., 1989). Hansen (1959) definite accessibility as a number of potential opportunities or the attractiveness of each 
destination multiplied by the cost of travel to the destination. Thus, high price properties are always associated with high accessibility, as the less time and money spent in travel. However, Adair et al. (2000) examine whether accessibility has a positive impact on housing value in the Belfast urban area. They measured the accessibility by employing straight-line distance to focal points such as the CBD. Results indicated the accessibility measured in this way was of little significance in explaining variation in house prices at a city -wide scale. However, many authors found that accessibility can be an important influence at sub-market level, particular in low-income areas. What is more, some studies show that housing and accessibility to employment centers are jointly chosen, in that those paying higher price are compensated by the lower cost of commuting to the CBD (see, So et al. 1997).

Accessibility is not a unitary concept however. Different people want to be close to different types of opportunities and different people value the costs of distance differently (Webster 2010). Some studies, have found that accessibility is connected with social neighborhood characteristics. Handy and Niemeir (1997), argue that different segments of the population care about different sets of opportunities and may evaluate the impedance to and attractiveness of opportunities in unique ways. Therefore, accessibility measures can also be disaggregated according to socioeconomic characteristics. For example, Wachs and Kumagai (1973) explored the social impacts of transportation decisions and the distribution of activities on different segments of the population as defined by income and occupation categories. Niemeier (1997) examined the worth of accessibility to people in different income segments. In general, some differentiation of individuals and households by selected characteristics should result in more accurate accessibility measures. However, the level of disaggregation has practical limits and is likely to show diminishing returns in terms of accuracy in housing market modeling and analysis. Similar statements were also made by Srinivasan and Ferreira (2002) and Wang (2001), with different social classes of people valuing accessibility differently. Thus, classifying 'raw' accessibility 
could reflect different class's perception for individual housing characteristics.

With regards to the type of accessibility, according to Webster (2010), accessibility in urban configuration can be considered as the pre-eminent urban public good and is differentiated according to supply and demand. He also notes that if it can be priced, it could be allocated more efficiently. Using network analysis of street configuration to more accurately measure the supply of general accessibility is an appealing idea since it makes good intuitive sense that street arrangement at multiple scales has a bearing on how a particular location is valued. Some empirical studies have confirmed that the space syntax "integration" has an impact on housing price using topological network measurements(Matthews and Turnbull 2007; Enström and Netzell 2008).Indeed, Hillier and Hanson (1984a) made the distinction that underlying structures of space carry patterns of behavior, which have a social function. Vaughan et al.(2005) and Vaughan and Penn (2006), employed space syntax network analysis to examine the relationship between urban form and immigrants quarters. Their results show that poorer immigrants tend to congregate in poverty areas with lower spatial connectivity. This logically will be reflected in the pattern of valuation and prices offered for such locations.

To summarize, although there is no consensus on the definition of a submarket, housing submarkets specifications can be classified into two categories: spatial and non-spatial methods of, approximating a segregated equilibrium market where the price per unit of housing and locational characteristics can be assumed constant. Spatial specifications rely on extant geographies while non-spatial methods allow geography to emerge from individual characteristics. However, the specifications discussed above are not efficient enough to deal with the cases of rapid urbanization in developing countries. This study will extend the non-spatial method by adding more emphasis on people's choice indirectly. I hypothesise that urban configuration features could be a new alternative specification for housing submarket delimitation, 
as accessibility is relevant to both spatial location and social economic status. Compared with traditional non-spatial specifications, specification by urban configurational features has three advantages: one is its spatial visualization ability; another is its greater degree of spatial accuracy and differentiation; and a third is its stability over time.

\subsection{Methodologies}

\subsubsection{Space syntax}

Space syntax radii segment analysis introduced by Hillier and Penn(2004) is employed in this study. The metric radii within which network measures are taken, overcomes the problems associated with earlier implementations of the method as noted in Chapter three. There are two important measurements for spatial accessibility, one is "integration" also known as "closeness", and another is called "choice" known as "betweeness", both already identified in Chapter three.

Furthermore, the setting of metric radii is same as it is in chapter four. The radii is mainly divided into three ranges, including walking scale, cycling or running scale, automobile scale. Moreover, it is noted that the optimal radius also depends on the study area according to Hillier and Penn (2004).

\subsubsection{Hedonic price model}

The hedonic price model function is same as in chapter four, following Orford's approach(1999), and despite a series of attributes on urban configuration, the building size, building types, tenure and social economic status would also be considered for this study. 


\subsubsection{Two-Step cluster analysis}

It is well known that clustering algorithms, k-means clustering and agglomerative hierarchical techniques, suffer from several issues (Bacher et al. 2004). The two-step cluster method is one of the so-called intelligent cluster analysis methods. The reason for adopting this method in this study is that different from traditional clustering techniques, the two-step analysis could deal with both categorical and continuous variables, and detect the optimal number of clusters automatically. This method groups the dataset for two procedures (1) pre-clustering the cases into many small sub-clusters; (2) clustering the result of step one to the desired number of cluster (SPSS 2001, 2004)

According to the developer Chiu, Fang, Chen, Wang and Jeris (2001), the aim of the pre-cluster step is to compute a new data matrix with fewer cases for the next steps, thus its cans the data records one by one, deciding the record should be merged or a new cluster based on the distance criterion (such as Log-likelihood distance and Euclidean distance)

The second step is to take the results of pre-clusters as input and cluster them into the desired number of clusters, which is based on hierarchical technique. Similar to the agglomerative hierarchical clustering method, two-step could determines the numbers of cluster automatically. A hierarchical clustering method merges stepwise and repeatedly until all the records are grouped into a single cluster. There are two distance measures based on the Euclidian distance and the log-likelihood distance. However, the Euclidian distance can be employed for continuous variables (SPSS 2001, 2004).

Log-likelihood distance can deal with both continuous and categorical variables(SPSS, 
2004). The Log-likelihood distance of two clusters is calculated by the natural logarithm of likelihood function. There are some assumptions for the log-likelihood distance implementation, for example, normal distributions of continuous variables, the categorical variables have multinomial distributions, and all variables should be independent of each other. The algorithm of Log-likelihood distance between cluster $\mathrm{i}$ and $\mathrm{j}$ is defined as:

$$
\begin{gathered}
d(i, j)=\xi_{i}+\xi_{j}-\xi_{(i, j)} \\
\xi_{s}=-N_{s}\left[\sum_{k=1}^{K^{A}} \frac{1}{2} \log \left(\hat{\sigma}_{k}^{2}+\hat{\sigma}_{s k}^{2}\right)+\sum_{k=1}^{K^{B}} \hat{E}_{s k}\right] \\
\hat{E}_{s k}=-\sum_{l=1}^{L_{K}} \frac{N_{s k l}}{N_{s}} \log \frac{N_{s k l}}{N_{s}}
\end{gathered}
$$

Where,

$d(i, j)$ denotes the distance between clusters $i$ and $j$;

$i, j$ represents the cluster formed by combining clusters $i$ and $j$;

$K^{A}$ is the total number of continuous variables;

$K^{B}$ is totalnumber of categorical variables;

$L_{k}$ is the number of categories for the $k^{\text {th }}$ categorical variable;

$N s$ denotes the total number of records in cluster $s$;

$N_{s k l}$ is the number of records incluster $s$ whose categorical variable $k$ takes $l$ category; $N_{k l}$ is the number of records incategorical variable $k$ that take the lcategory; $\hat{\sigma}_{k}^{2}$ is the estimated variance of the continuous variable $k$ for the entire dataset; $\hat{\sigma}_{s k}^{2}$ denotes the variance of the continuous variable $k$ in cluster $j$.

In order to determine the number of clusters, two indicator of Schwarz's Bayesian Information Criterion (BIC) and Akaike's Information Criterion (AIC) could provide robust estimation for numbers of cluster. For $j$ clusters, the two indicators are computed as follows:

$$
\begin{gathered}
B I C(J)=-2 \sum_{j=1}^{J} \xi_{j}+m_{J} \log (N), \\
A I C(J)=-2 \sum_{j=1}^{J} \xi_{j}+2 m_{J}
\end{gathered}
$$




$$
m_{J}=J\left\{2 K^{A}+\sum_{k=1}^{K^{B}}\left(L_{k}-1\right)\right\}
$$

In short summary, compared with traditional cluster technique, two-step clustering analysis can incorporate with both continuous and categorical data, and it seems has high potential for housing submarket studies, in particular dealing with the category variable of the dwelling type. The analysis was computed using SPSS 16.

\subsubsection{Chow test}

The Chow test (Chow 1960) tests the regression equation c evaluates the parameter equality by F-test in struck break. The concept can be expressed as follows:

$$
\begin{gathered}
y_{t}=\alpha_{0}+\alpha_{1} x_{t}+\mu_{t} \\
y_{1 t}=\beta_{1}+\beta_{2} x_{t}+\mu_{1 t} \\
y_{2 t}=\delta_{1}+\delta_{2} x_{t}+\mu_{2 t}
\end{gathered}
$$

Where suggests if the parameters in the above models are the same, i.e. $\beta_{1}=$ $\delta_{1}, \beta_{2}=\delta_{2}$, then models $y_{1 t}$ and $y_{2 t}$ can be expressed as a single model $y_{t}$, where there is a single regression line.

The F-test statistic from the following formula:

$$
\mathrm{F}=\frac{\left(\mathrm{RSS}_{\mathrm{c}}-\mathrm{RSS}_{\mathrm{i}}-\mathrm{RSS}_{\mathrm{j}}\right)(\mathrm{n}+\mathrm{m}-2(\mathrm{k}+1))}{(\mathrm{k}+1)\left(\mathrm{RSS}_{\mathrm{i}}+\mathrm{RSS}_{\mathrm{j}}\right)}
$$

Where

$\mathrm{n}$ and $\mathrm{m}$ denotes the numbers of sample in the sub group $i$ and $j$ respectively; $\mathrm{RSS}_{\mathrm{c}}$ is the residual sum of squares of the combined model ; 
It is noted that the critical value $\mathrm{F}_{\mathrm{cv}}$ which is distributed as follows: $\mathrm{F}_{\mathrm{cv}} \sim F[K+$ $1, n+m-2(k+1)]$ with a $\mathrm{k}+1$ numerator and $\mathrm{n}+\mathrm{m}-2(\mathrm{k}+1)$ denominator degrees of freedom.

The Chow test was conducted using Eview 6.0.

\subsubsection{Weighted standard error estimation}

The weighted standard error estimation is computed to compare the accuracy of the price estimates generated where submarkets are identified with those derived from a single model covering the entire market (see, Schnare and Struyk, 1976; Tu et al., 2007).

$$
\mathrm{SE}_{\mathrm{c}}=\frac{\mathrm{N}_{1}-\mathrm{k}_{1}-1}{\sum\left(\mathrm{N}_{\mathrm{j}}-\mathrm{K}_{\mathrm{j}}-1\right)} \mathrm{SE}_{1}+\frac{\mathrm{N}_{2}-\mathrm{k}_{2}-1}{\sum\left(\mathrm{N}_{\mathrm{j}}-\mathrm{K}_{\mathrm{j}}-1\right)} \mathrm{SE}_{2}+\ldots \ldots \ldots \ldots+\frac{\mathrm{N}_{\mathrm{j}}-\mathrm{k}_{\mathrm{j}}-1}{\sum\left(\mathrm{N}_{\mathrm{j}}-\mathrm{K}_{\mathrm{j}}-1\right)} \mathrm{SE}_{\mathrm{j}} \text { Equation (5.9) }
$$

Where

$\mathrm{N}_{\mathrm{j}}$ is the number of transactions in the jth submarket,

$\mathrm{k}_{\mathrm{j}}$ is the number of the explanatory variables in the $\mathrm{jth}$ submarket

\subsection{Study area and dataset}

The study area and data source are exactly same as mentioned in chapter four, taking a part of Cardiff metropolitan area as the case. 


\subsection{Empirical analysis}

The main purpose of this research is to explore the housing submarket segmentations of Cardiff by two alternative specifications of accessibility at the urban street level. The first specification is to construct the submarket only by best-fit spatial urban configuration characteristics at a certain radii. The second specification is to identify housing submarket by all urban configuration characteristics and building structure characteristics. The reason for testing these two methods against each other is to compare the how improvement of results when traditional determinants for people's preference are considered. As theory above suggest the spatial locational information plus building types has the best performance to identify the sub-markets in UK context.

According to the housing submarket literature, the application of hedonic models for housing submarkets normally follows Schnare and Struyk (1976)'s three-step procedures. The first step is an estimation of the entire market model. In the second step, hedonic regressions are estimated separately for the individual submarkets, which are divided into segments by either prior geography or data driven specifications. The final step is to use the Chow test and the standard error (SE) to test the existence of statistically significant submarkets and to discover the efficient number of submarkets, with the $\mathrm{F}$ test determining whether the resulting reduction in sum of squared residuals is significant. If the reduction is significant, it indicates the specification for submarket is appropriate (Goodman and Thibodeau 2003).

\subsubsection{Market-wide hedonic model}

In order to get a robust model for the market-wide estimations, a range of structure, neighborhood, and locational characteristics are considered, amounting to 20 variables. An additional 14 models are constructed to examine at what scale 
accessibility has the greatest impact on housing property value. Generally, the results in table (5.1) show that all models are statistically significant; the adjust R-square of all models are above $61 \%$; the $\mathrm{F}$ test of all model are significant at $5 \%$ confidence level, and issues of multicollinearity are absent in 15 models in which the VIF of each variable is under 10. Apace syntax integration, and choice measures at all radii are significant, thus, it is concluded that the urban configurational features do in fact influence property values, ceteris paribus.

Some interesting findings from table (5.1) and figure (5.1) are highlighted.

Firstly, the best performance model for market-wide estimation is the model combined with accessibility index at radii $7 \mathrm{~km}$, (the adjust-R square is 0.633 and the $\mathrm{F}$ test is also significant at 5\% confidence level).Having removed the insignificant variables, the final standard error of the model is 1942.597, and there are 21 significantly variables.

Secondly, it is found that the space syntax accessibility index at all radii are significant, and integration always has a positive impact, whereas, choice always shows a negative sign. This can reflect that higher value properties located in places where the street segments are connected well to destination opportunities but with lower traffic volume. In addition, the t-value of both integration and choice are influenced with the increase of the radii, which stabilizes with small fluctuations at radii $2.5 \mathrm{~km}$ and finally decreasing after radii $7 \mathrm{~km}$. The t-value of both integration and choice reached a peak at -10.040 and 28.163 respectively, which indicates that the urban configurational features at radii $7 \mathrm{~km}$ have the strongest impact on housing price.

Finally, it is found that urban configurational features can interact with social neighborhood and locational characteristics, as the t-value of some variables became 
insignificant or unstable when the models were combined with accessibility at different scale levels. Compared with other variables, the dummy variables of transaction year are very stable, as shown in figure (5.1). The t-value of building types all slightly increased, which implies that there is no correlation between urban configuration and building types in this study area. However, the t-value of the floor area decreases slightly and starts to increase after radii $3 \mathrm{~km}$. Neighborhood characteristics is the variable mostly affected by urban configurational features at different levels. For example, the t-value of people from the "blue-collar" group became insignificant once urban configurational attributes are considered. The t value of the remaining groups firstly increased before radius $2500 \mathrm{~m}$, then decreased slowly, reaching its lowest rate at radii at $7 \mathrm{~km}$. With respect to the locational attributes, the t-value of the distance to $\mathrm{CBD}$ reduced as space syntax radii increased, but remained significant at the $5 \%$ confidence level. Thus, I can say that accessibility contained in urban configuration appears to incorporate effects of both locational characteristics and social economics characteristics. 
n

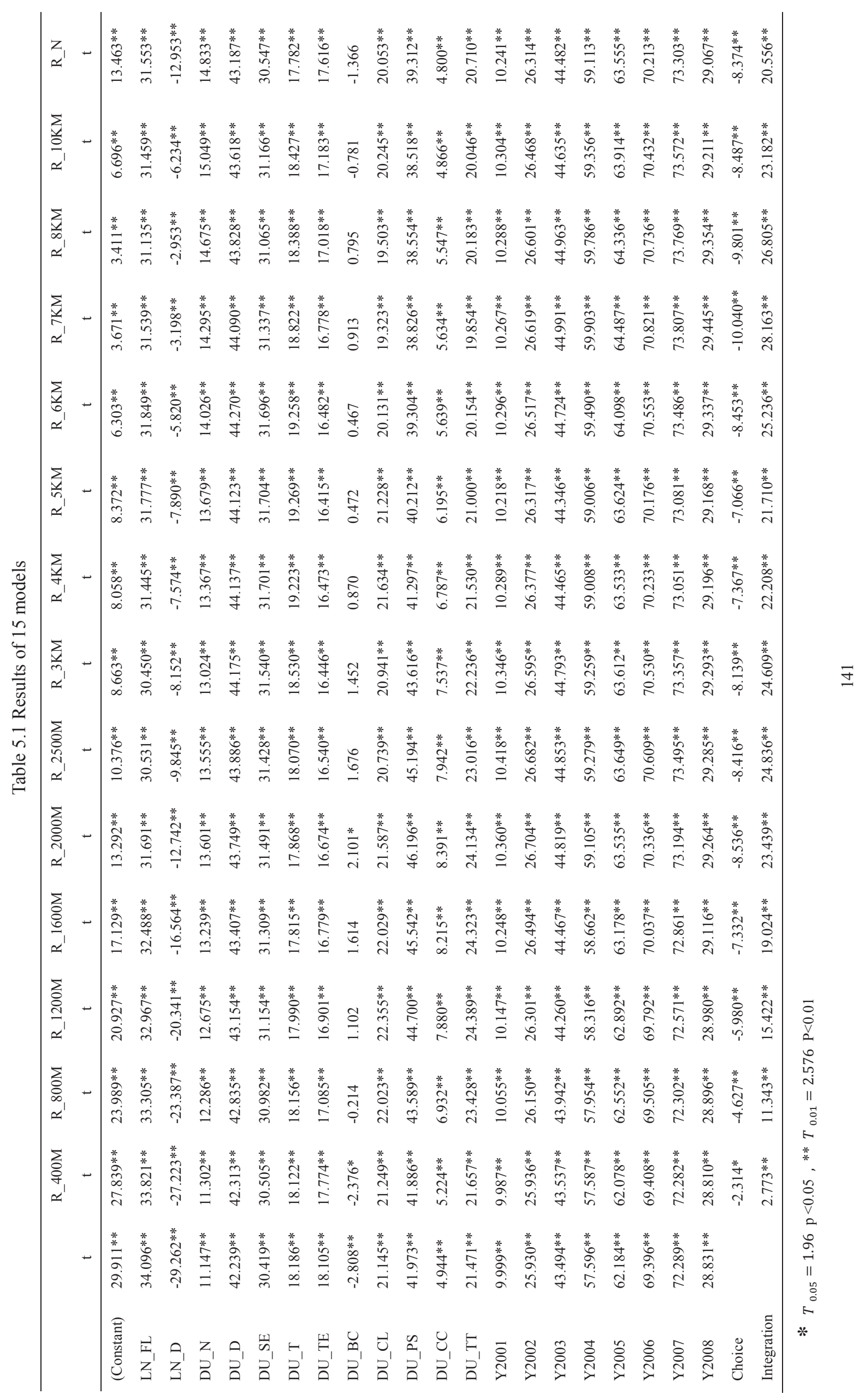




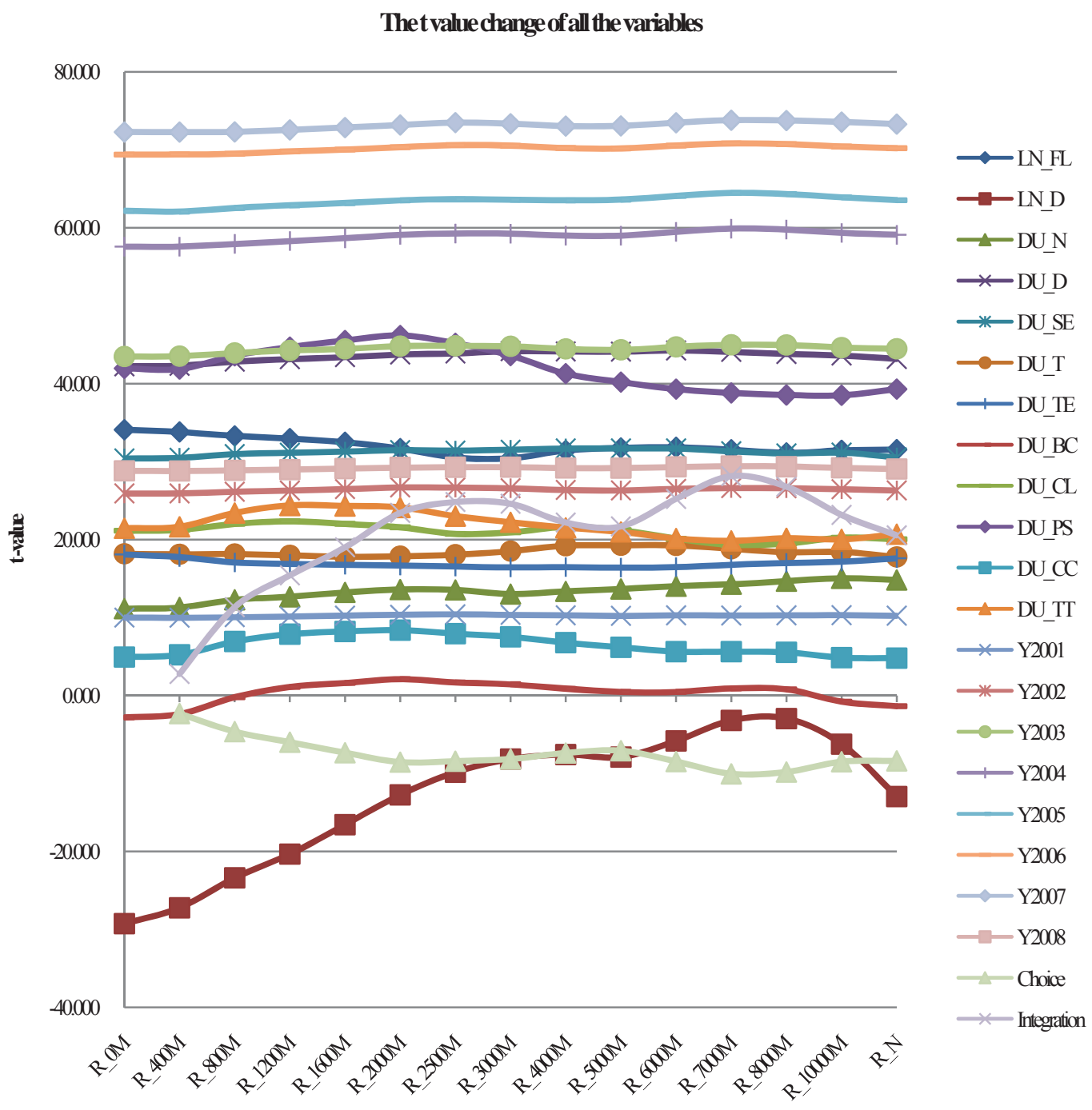

Figure 5.1 The t value change of all the variables via 15 models

Source: author

\subsubsection{Specifications and estimations for submarkets}

The aim of this study is to examine the efficiency of specifying housing submarkets using accessibility of urban street layouts as the main indicator. Thus, it is necessary to compare the results with traditional specifications (dwelling types and nested spatial and dwelling types), which are widely accepted in the housing submarket studies of the UK. Firstly, for each specification, hedonic regressions are estimated separately for each submarket. Then the chow test is applied to examine whether submarket exists, and the number of submarkets by asking whether the coefficient of 
each attribute in different submarket is equal. Finally, the weighted standard error test is used to examine the improvement of the sub-market estimation.

\subsubsection{Dwelling type specification}

Table 5.2 Estimation results of dwelling type specification

\begin{tabular}{|c|c|c|c|c|c|c|c|c|}
\hline & \multicolumn{2}{|c|}{ Terrace } & \multicolumn{2}{|c|}{ Semidetached } & \multicolumn{2}{|c|}{ Detached } & \multicolumn{2}{|c|}{ Flat } \\
\hline $\mathrm{N}$ & \multicolumn{2}{|c|}{8974} & \multicolumn{2}{|c|}{3598} & \multicolumn{2}{|c|}{1715} & \multicolumn{2}{|c|}{2920} \\
\hline $\begin{array}{l}\text { Adjust } \\
\text { R-square }\end{array}$ & \multicolumn{2}{|c|}{0.578} & \multicolumn{2}{|c|}{0.654} & \multicolumn{2}{|c|}{0.591} & \multicolumn{2}{|c|}{0.638} \\
\hline F test & \multicolumn{2}{|c|}{662.083} & \multicolumn{2}{|c|}{358.349} & \multicolumn{2}{|c|}{115.747} & \multicolumn{2}{|c|}{272.082} \\
\hline \multirow[t]{2}{*}{ RSS } & \multicolumn{2}{|c|}{963.592} & \multicolumn{2}{|c|}{320.484} & \multicolumn{2}{|c|}{186.696} & \multicolumn{2}{|c|}{315.488} \\
\hline & $\mathrm{t}$ & Sig. & $\mathrm{t}$ & Sig. & $\mathrm{t}$ & Sig. & $\mathrm{t}$ & Sig. \\
\hline (Constant) & 1.235 & 0.217 & 6.849 & $0.000 * *$ & 2.352 & $0.019 *$ & 1.238 & 0.216 \\
\hline LN_FL & 24.844 & $0.000 * *$ & 21.904 & $0.000 * *$ & 23.663 & $0.000 * *$ & 14.430 & $0.000 * *$ \\
\hline LN_D & $-0.887 *$ & 0.375 & -6.590 & $0.000 * *$ & -2.248 & $0.025 *$ & $-1.046^{*}$ & 0.296 \\
\hline DU_N & 2.781 & $0.005 * *$ & 9.951 & $0.000 * *$ & 2.126 & $0.034 *$ & 15.455 & $0.000 * *$ \\
\hline DU_TE & 13.719 & $0.000 * *$ & 8.304 & $0.000 * *$ & 13.389 & $0.000 * *$ & 5.171 & $0.000 * *$ \\
\hline DU_CL & 14.851 & $0.000 * *$ & 8.639 & $0.000 * *$ & 5.479 & $0.000 * *$ & 6.629 & $0.000 * *$ \\
\hline DU_PS & 12.249 & $0.000 * *$ & 26.534 & $0.000 * *$ & 15.323 & $0.000 * *$ & 19.342 & $0.000 * *$ \\
\hline DU_CC & 3.648 & $0.000 * *$ & 3.560 & $0.000 * *$ & 2.281 & $0.023 *$ & $1.177^{*}$ & $0.239 * *$ \\
\hline DU_TT & 16.657 & $0.000 * *$ & 13.156 & $0.000 * *$ & 6.028 & $0.000 * *$ & 5.558 & $0.000 * *$ \\
\hline Y2001 & 8.453 & $0.000 * *$ & 5.067 & $0.000 * *$ & 3.140 & $0.002 * *$ & 4.226 & $0.000 * *$ \\
\hline Y2002 & 21.576 & $0.000 * *$ & 13.545 & $0.000 * *$ & 5.949 & $0.000 * *$ & 11.886 & $0.000 * *$ \\
\hline Y2003 & 37.994 & $0.000 * *$ & 22.406 & $0.000 * *$ & 10.066 & $0.000 * *$ & 16.896 & $0.000 * *$ \\
\hline Y2004 & 47.912 & $0.000 * *$ & 29.451 & $0.000 * *$ & 13.389 & $0.000 * *$ & 23.565 & $0.000 * *$ \\
\hline Y2005 & 50.464 & $0.000 * *$ & 31.807 & $0.000 * *$ & 16.823 & $0.000 * *$ & 27.309 & $0.000 * *$ \\
\hline Y2006 & 56.828 & $0.000 * *$ & 34.536 & $0.000 * *$ & 17.075 & $0.000 * *$ & 28.280 & $0.000 * *$ \\
\hline Y2007 & 58.689 & $0.000 * *$ & 35.497 & $0.000 * *$ & 19.014 & $0.000 * *$ & 29.400 & $0.000 * *$ \\
\hline Y2008 & 24.096 & $0.000 * *$ & 15.606 & $0.000 * *$ & 6.418 & $0.000 * *$ & 11.116 & $0.000 * *$ \\
\hline CH_R7000M & -7.982 & $0.000 * *$ & -3.174 & $0.002 * *$ & 1.532 & 0.126 & -7.366 & $0.000 * *$ \\
\hline INT_R7000M & 19.305 & $0.000 * *$ & 9.957 & $0.000 * *$ & 1.594 & 0.111 & 11.917 & $0.000 * *$ \\
\hline NSV & 1 & & & & & & & \\
\hline
\end{tabular}

Dwelling type specification is the simplest way to identify submarkets in the UK, as it assumes that people have homogenous perceptions for each building type. In the UK, there is a strong general cultural preference for detached over semi-detached, semiover terraced and terraced over apartments. As the dataset of this study area has four types (detached house, semidetached house, flat and terrace house), the whole market is easily classified into four submarkets. Table (5.2) shows the estimation results for each submarket, and several interesting findings are summarized as follows. Firstly, 
compared with the market-wide model, all models for submarkets are statistically significant and the sign of each attribute in all models is as expected. There is no serious multicollinearity issue in any of the models, but not all the adjusted R-squares for the submarket models have experienced an increase compared to the whole market model, which varies from 0.578 to 0.654 . Secondly, it is found that the locational characteristic (distance to $\mathrm{CBD}$ ) is insignificant for both terrace house and flat submarkets, which indicates that when people choose terrace house and flat, they do not care about how far the properties are located from the CBD. This seems reasonable since in the study area most of the terrace houses are located near the city center, whereas, most of the flats are far from the city center. Thirdly, compared with the attribute of distance to $\mathrm{CBD}$, the accessibility index of urban configuration is more stable, and both integration and choice are only insignificant in the detached housing submarket. It indicates that people, who chose large houses are relying on the car and only consider how far it is from city center. They do not care whether the street segment connects well and if traffic volume is high. Furthermore, the results suggest that the street layout features contribute to the highest t-value for terrace houses, even if it is close to the city center, which implies that accessibility provides extra spatial impact on property value. Finally, most of the social economic classes are significant in all submarkets, with the only exception being people "constrained by circumstances" in flat submarket, which reflects it indicate that people do not want to buy flats in poor neighborhoods.

Table 5.3 Chow test results of dwelling type specification

\begin{tabular}{lc}
\hline \multicolumn{1}{c}{ Segments } & Chow \\
\hline Flat with detached & $598.5615^{* *}$ \\
Flat with semidetached & $604.5928^{* *}$ \\
Flat with terraced & $279.4152^{* *}$ \\
Detached with semidetached & $677.8311^{* *}$ \\
Detached with terraced & $333.2813^{* *}$ \\
Semidetached with terraced & $292.0371^{* *}$ \\
\hline$F_{(20, \mathrm{n}-2 \mathrm{k})}=1.878 \mathrm{a}=0.01$ &
\end{tabular}

Table (5.3) shows the results of the chow tests on the dwelling type specified submarkets. The hypothesis is that implicit price of individual attributes are equal between any two submarkets. All chow test results are significant at $1 \%$ confidence level, which shows that there are significant differences for the implicit prices between the four potential submarkets. Thus, the submarkets can be taken to exist and 
the implicit price for attributes vary systematically across these four equilibrium submarkets.

\subsubsection{Nested spatial and dwelling types specification}

Nested Spatial and dwelling type specification is another method for identifying submarkets and its concept rests on the assumption that when people locate, they are concerned about both spatial and building type characteristics, especially in large cities. This is because sometimes there are multi-employment centers, and people have different origins and destinations. However, this type of specification is ad hoc-based, requiring prior knowledge of social spatial structure in the city. In regards to the issue of pre-defined spatial boundary, the normal approach is to use natural boundaries such as rivers, rail-lines and motorways. In this study, the natural boundary of the A48 motorway divides the study area into two segments equally (north and south parts) and there is clear prima facie evidence that these operate as separate housing markets. This division is then combined with structure characteristics, which categorizes the housing market into eight potential submarkets.

Table (5.4) shows the regression results for all eight potential housing submarkets. Key findings are summarized as follows. Firstly, all models are significant and pass the $\mathrm{F}$ test. By comparing the results of the dwelling types specification, the fitness for each model is improved, as the adjust R-square is within the range of 0.568 to 0.705 . Secondly, the impact of locational attributes (the distance to CBD) and floor area are unstable across all submarkets. For example, in the southern part, it is found that the signs of the distance to CBD became positive and are insignificant in most submarkets except for the submarket of flats and floor area, which contribute to the highest $\mathrm{t}$-value in the southern part. This indicates that within an area proximate to the city center, the floor area is the main determinant for people purchasing detached, semidetached and terrace house, rather than the factor of short distance to CBD. In contrast, in the northern part, the sign of distance to CBD is negative and the t-value of floor area becomes smaller, which suggests that in the suburban area, people are more concerned about the transportation costs than the property size. Thirdly, although the signs of space syntax accessibility index in all models are expected, 
some urban configuration features are not constant across all models as before. For example, it is found that integration in all models is positive and is significant in seven submarkets except for the submarket of detached housing. However, the sign of choice is still negative and it becomes insignificant in the submarket of detached housing within the southern part, and terrace, semidetached, and detached housing in the northern part, which implies that the location with less traffic flow is not associated with high value properties in these submarkets. Fourthly, the social economic class "constrained by circumstances" is only significant at $5 \%$ confidence level in the submarkets of semidetached and flat in the south part, and terrace housing in the north part, which reflects the specific preference of people in these classes.

Table (5.5) shows the results of the parameter equality test for the prior specification of nested spatial and dwelling types. Again, the chow tests for any two submarkets is statistically significant at $1 \%$ confidence level, which provides evidence for the existence of eight statistically significant submarkets in this study area. 


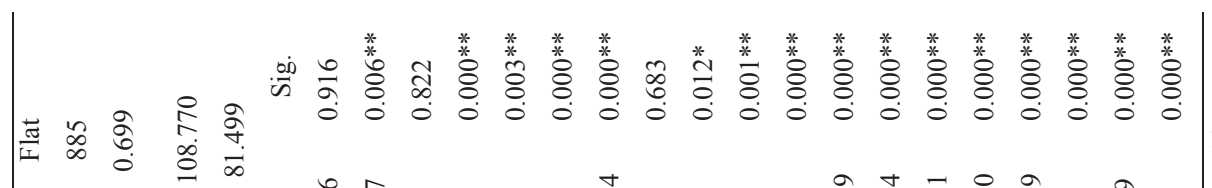

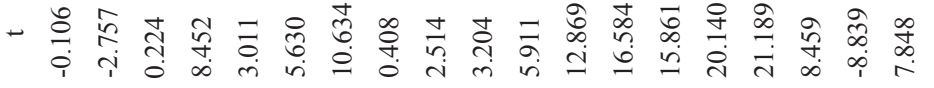

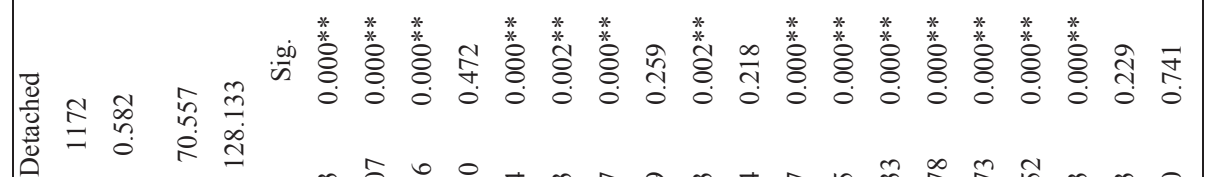

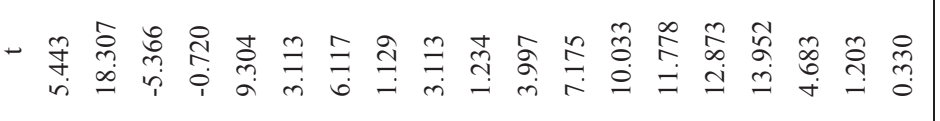

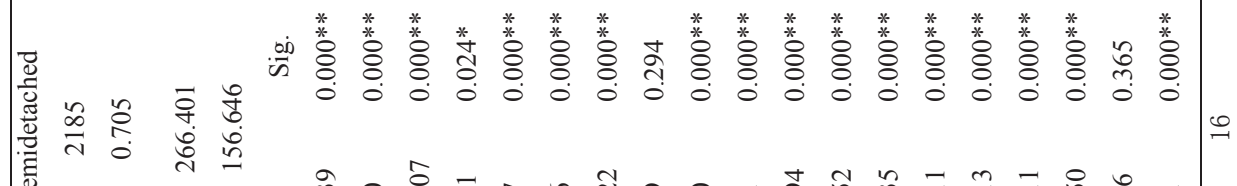

O2

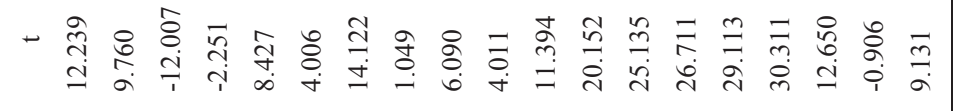

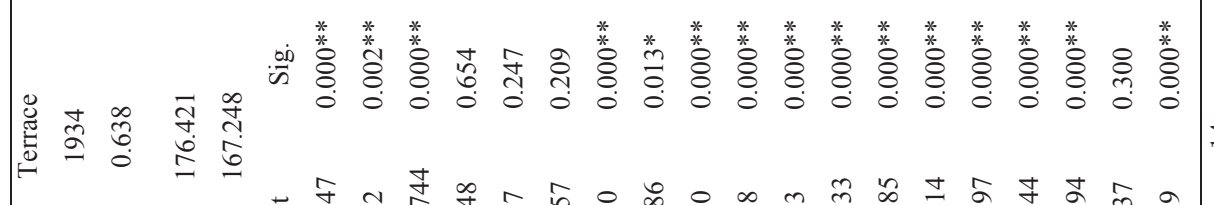

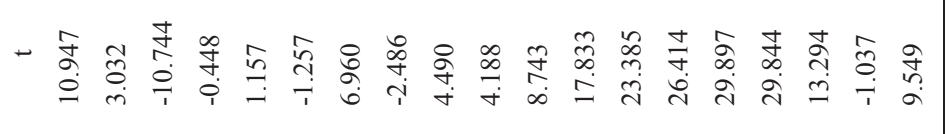

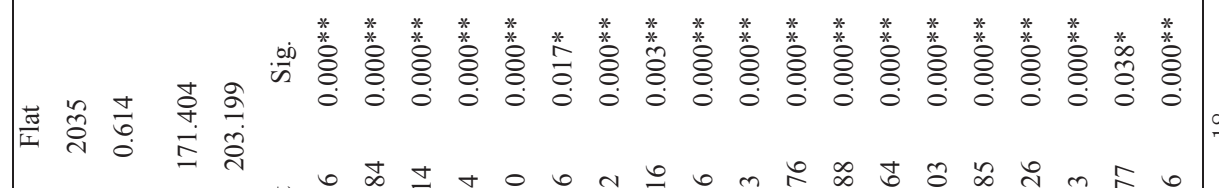

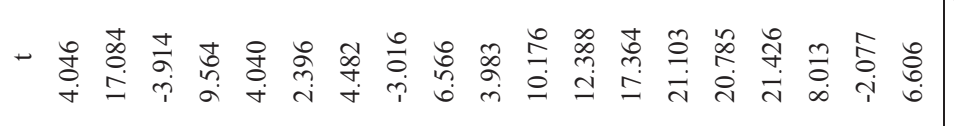

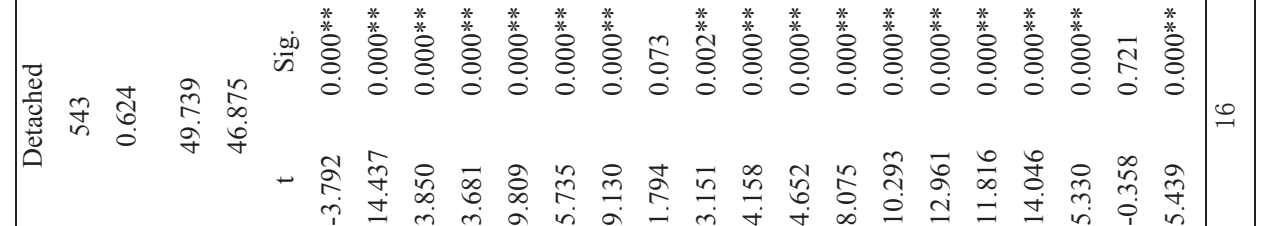
言

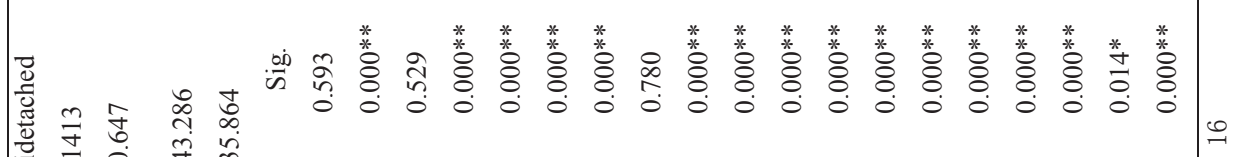

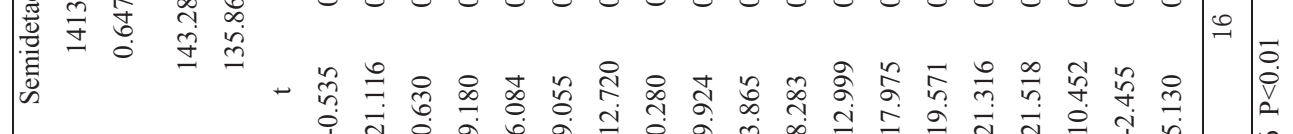

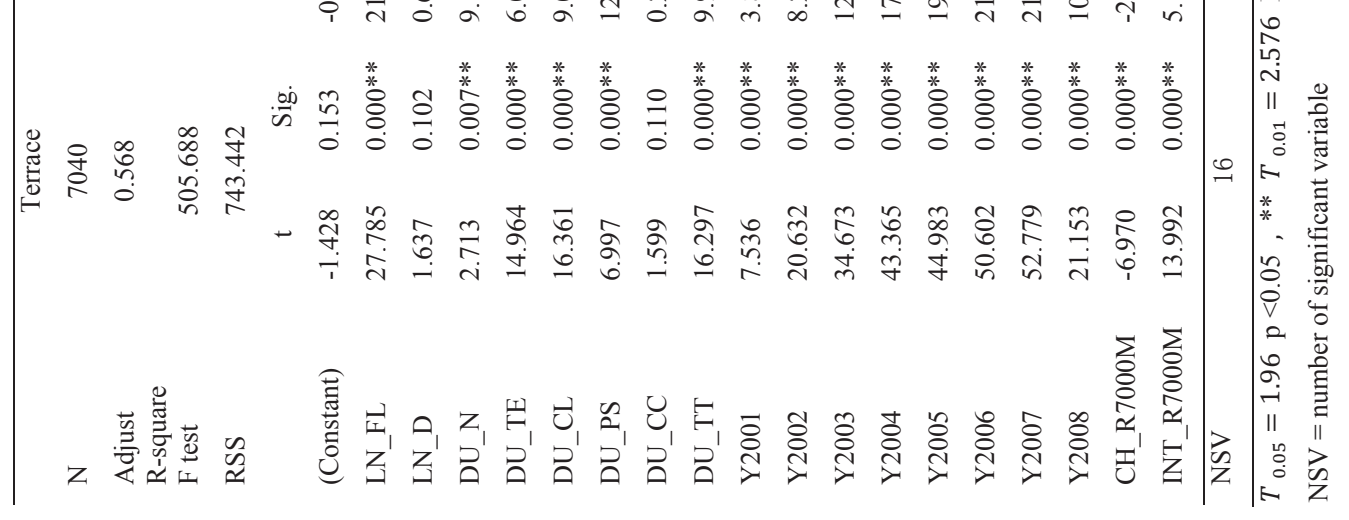


Table 5.5 Chow test results of spatial nested specification

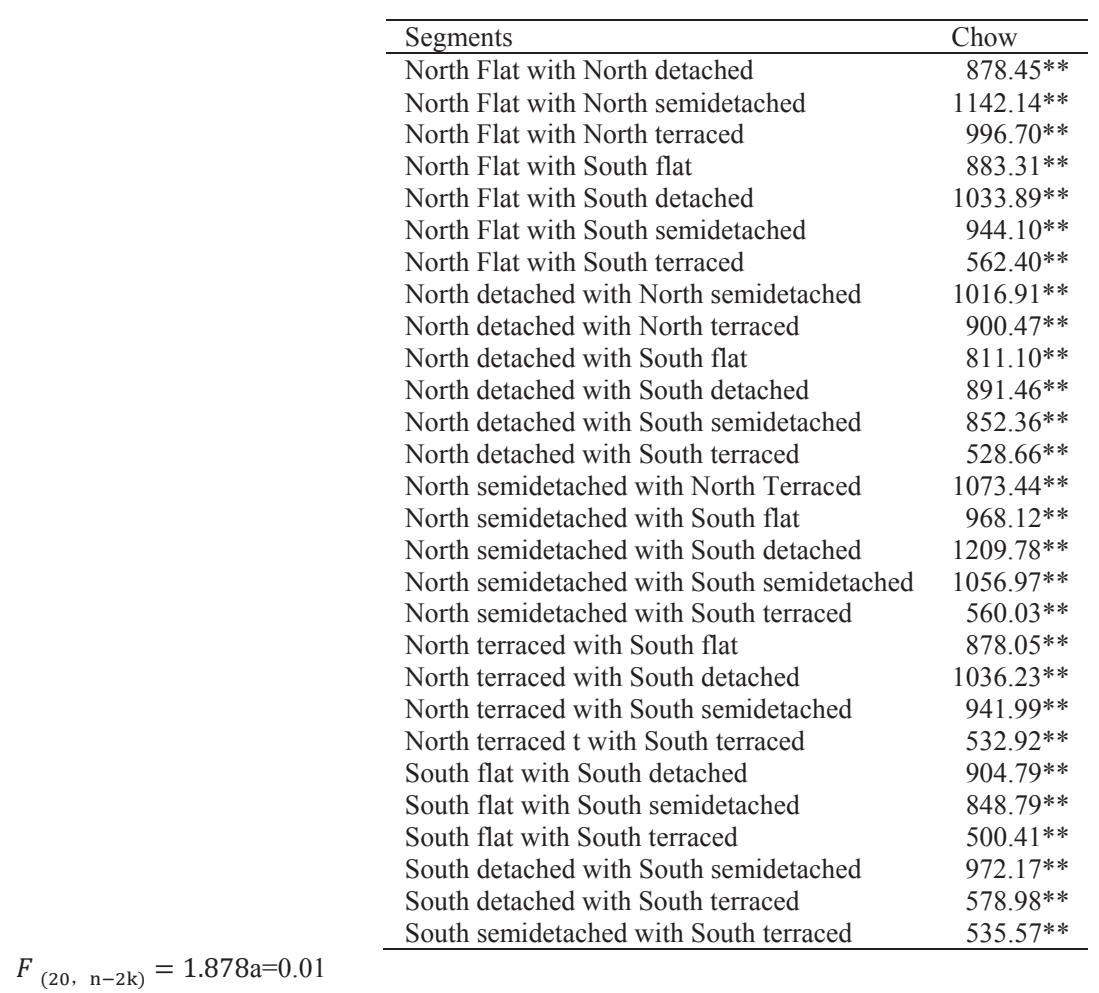

\subsubsection{Optimal urban configurational features specification}

Two alternative specifications for housing submarkets are based on urban configuration features in the market-wide model. The first specification is only considered with space syntax spatial index at $7000 \mathrm{~m}$ radius (having established that this is the radii at which space syntax measures have strongest influence on house prices), by the two-step clustering analysis, which can automatically acquire the optimal numbers of clusters. Table (5.6) shows the results of clustering. It can be clearly seen that with the increase of numbers of clusters, the BIC criterion decreases steadily. However, in places where there are four clusters, the ratio of distance is the highest of all at 2.084. Thus, the results confirm that there are four potential housing submarkets using this method. Table (5.7) shows the descriptive statistics of each submarket. 
Table 5.6Cluster results of optimal urban configurational features specification

\begin{tabular}{|c|c|c|c|c|}
\hline \multicolumn{5}{|c|}{ Auto-Clustering } \\
\hline Number of Clusters & $\begin{array}{c}\text { Schwarz's Bayesian } \\
\text { Criterion (BIC) }\end{array}$ & BIC Changea & $\begin{array}{c}\text { Ratio of BIC } \\
\text { Changesb }\end{array}$ & $\begin{array}{c}\text { Ratio of Distance } \\
\text { Measuresc }\end{array}$ \\
\hline 1 & 23891.979 & & & \\
\hline 2 & 13049.078 & -10842.901 & 1.000 & 1.793 \\
\hline 3 & 8064.271 & -4984.807 & 0.460 & 1.153 \\
\hline 4 & 6297.923 & -1766.349 & 0.163 & 2.084 \\
\hline 5 & 4583.364 & -1714.558 & 0.158 & 1.006 \\
\hline 6 & 4620.152 & 36.788 & -0.003 & 1.365 \\
\hline 7 & 4497.368 & -122.784 & 0.011 & 1.215 \\
\hline 8 & 4127.028 & -370.340 & 0.034 & 1.008 \\
\hline 9 & 3846.759 & -280.269 & 0.026 & 1.025 \\
\hline 10 & 3871.099 & 24.340 & -0.002 & 1.182 \\
\hline 11 & 3480.348 & -390.751 & 0.036 & 1.146 \\
\hline 12 & 2623.182 & -857.166 & 0.079 & 1.001 \\
\hline 13 & 2613.258 & -9.924 & 0.001 & 1.211 \\
\hline 14 & 2632.163 & 18.905 & -0.002 & 1.015 \\
\hline 15 & 2644.196 & 12.033 & -0.001 & 1.177 \\
\hline
\end{tabular}

a. The changes are from the previous number of clusters in the table.

b. The ratios of changes are relative to the change for the two cluster solution.

c. The ratios of distance measures are based on the current number of clusters against the previous number of clusters.

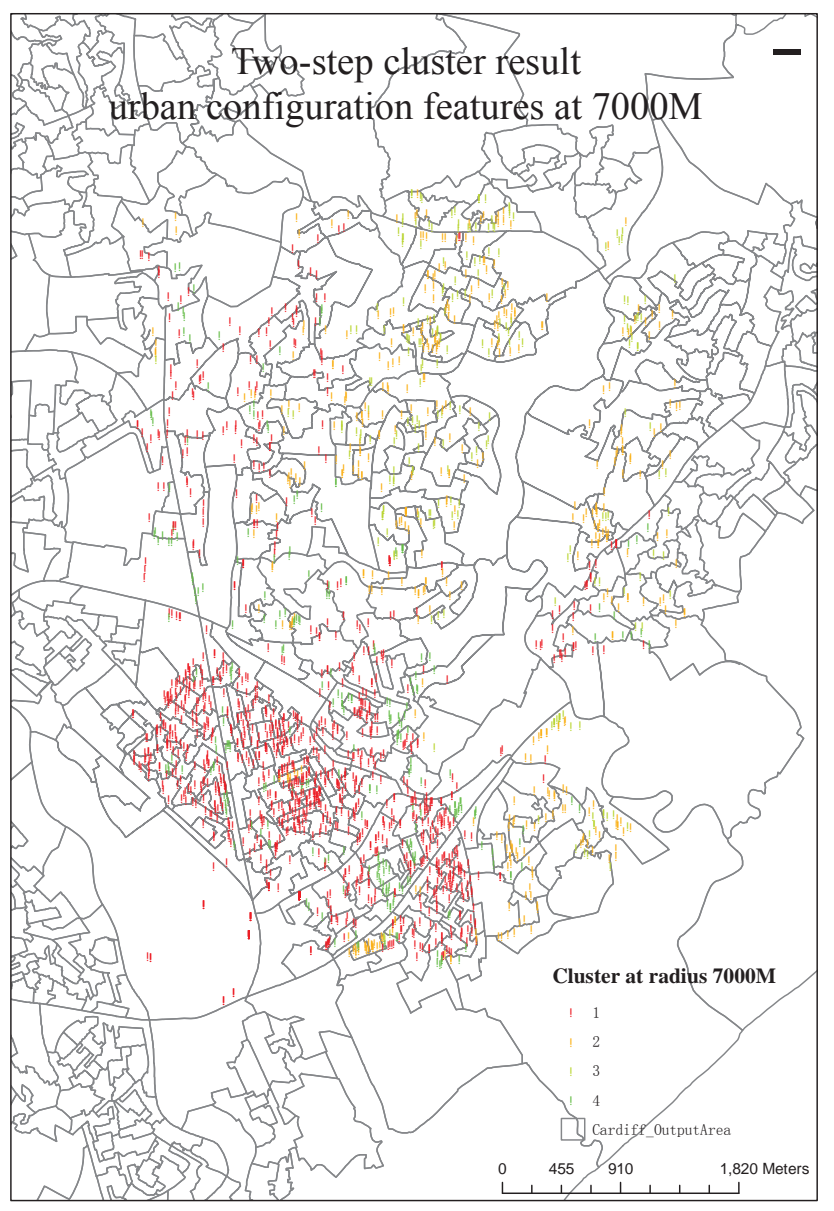

Figure 5.2 Two-step cluster result of urban configuration features at $7 \mathrm{~km}$

Source: author

Figure (5.2) shows the pattern of different of urban configurational features that are 
associated with different spatial properties. It can be seen that submarket number one has the highest value of both integration and choice, which might indicate that the properties tend to be situated alongside the main streets, whilst submarket number three has the lowest value of both integration and choice, which indicates that the properties are located close to the boundaries of the study area. The properties of submarket number four are mainly located at the city center but with dead-end roads, which is why its integration is still high but the choice is low. Finally, the remaining properties are in submarket number two, where integration is second lowest and choice is second highest. In addition, there seems to be a clear boundary between two parts, which indicates that the structure of spatial accessibility of urban configuration is close to Hoyt's sector model(1939) rather than linearly diffusion concentric zone model.

In addition, further examination of each submarket reveals that there are specific trends of personal preference for urban configuration in the different markets. For example, it is found that submarket one has the largest observations of all and comprises $53.589 \%$ of all houses in the area where above $50 \%$ of properties are terrace houses. Furthermore, in submarket one, the average housing price and floor area are highest of all. In terms of social economic status, in submarket one, most people are living in the city and other typical characteristics. In contrast, there are just $11.95 \%$ of total observations in submarket three, which has the cheapest average housing price and smallest average floor area. The proportions of different social economic status groups in submarket two and three are similar, as around one third of people are from the blue collar group and less people are from 'living in the city' group, which also confirms that the demand for integration is more associated with social economic status. As expected submarket four contains properties on the central streets but not on the high street, which has the second largest average housing price and third lowest floor areas. It is noted that the largest portions of social classes found in submarket four are from city living and multiple culture groups. With regards to the distribution of dwelling types, most detached and semidetached house are found in submarkets two and three, which both have lower value in terms of space syntax integration, which in turn can also indicate that the further away from the city the bigger the size of the house. Alternatively, as I have noted already, it may be that more 
wealthy people living in large suburban houses with good car-born mobility have less of a demand for high street network connectivity. 


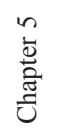

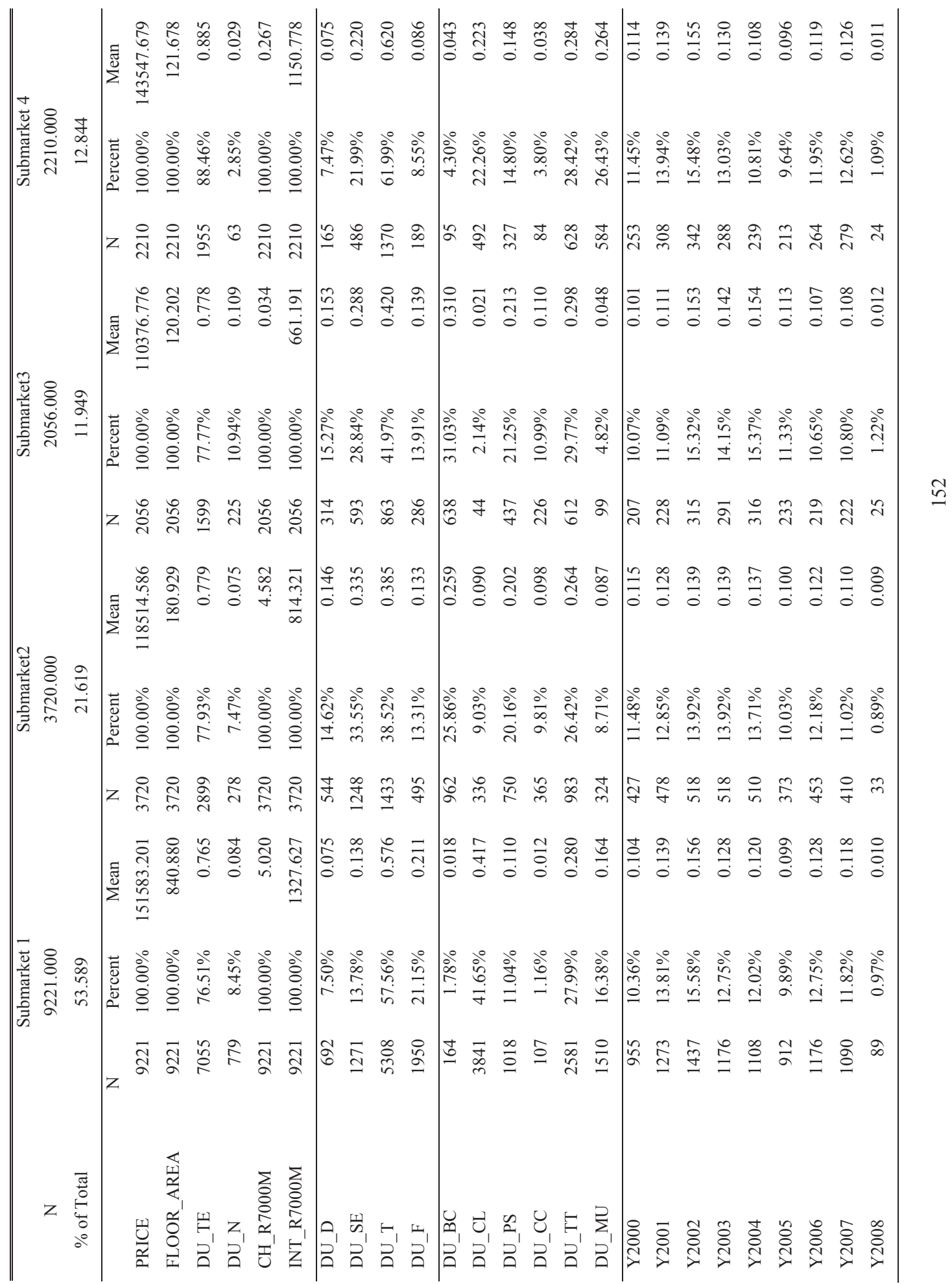


Table (5.8) shows the results of four submarkets estimations specified by different combination of high and low value of integration and choice at $7 \mathrm{~km}$ radii. The reason for doing this is insight how different social class are associated with different levels of spatial accessibility.

Key findings are highlighted in the following. Firstly, the model for each submarket is significant, and the fitness for each model seems improved, as the adjusted R-square ranges from 0.586 to 0.719 . Secondly, distance to $\mathrm{CBD}$ is also significant in all submarkets, but only with a positive sign in the submarket with the highest value of both integration, and choice. This might indicate that if the street layout is well designed in terms of accessibility, transportation cost could be ignored. Thirdly, with regards to configurational features, integration at radii $7 \mathrm{~km}$ is always significant with a positive sign in all submarkets. In the submarket which has high integration and the lowest choice, it is found that choice is insignificant because the properties of this submarket are alongside the main street or near the city center but with dead-end street segments. Moreover, choice is found to be significant but with a positive sign in the submarket which is lowest both in terms of integration and choice. Properties in this submarket are communities in suburb urban areas; the high choice value has a positive impact and the reason maybe because it is associated with low crime rate. Fourthly, since the people of "constrained by circumstances" have a small portion in submarket two and three, where the street segments are less connected, it is expected to be insignificant. Moreover, all classes are significant in all submarket at 5\% confidence level, which provides the evidence that social economic status can also impact on the submarket specified by urban configuration features. Finally, the results of Chow tests are shown in table (5.9). All six F-statistics are significant at the 0.1 level and the null hypotheses are rejected with a high level of confidence, confirming the existence of four submarkets. 
Table 5.8 Estimation results of optimal urban configuration specification

\begin{tabular}{|c|c|c|c|c|c|c|c|c|}
\hline & \multicolumn{2}{|c|}{ Submarket 1} & \multicolumn{2}{|c|}{ Submarket 2} & \multicolumn{2}{|c|}{ Submarket3 } & \multicolumn{2}{|c|}{ Submarket 4} \\
\hline $\mathrm{N}$ & \multicolumn{2}{|c|}{9221} & \multicolumn{2}{|c|}{3720} & \multicolumn{2}{|c|}{2056} & \multicolumn{2}{|c|}{2210} \\
\hline $\begin{array}{l}\text { Adjust } \\
\text { R-square }\end{array}$ & \multicolumn{2}{|c|}{0.586} & \multicolumn{2}{|c|}{0.711} & \multicolumn{2}{|c|}{0.719} & \multicolumn{2}{|c|}{0.643} \\
\hline$F$ test & \multicolumn{2}{|c|}{601.324} & \multicolumn{2}{|c|}{396.022} & \multicolumn{2}{|c|}{221.740} & \multicolumn{2}{|c|}{189.305} \\
\hline \multirow[t]{2}{*}{ RSS } & \multicolumn{2}{|c|}{1180.303} & \multicolumn{2}{|c|}{299.322} & \multicolumn{2}{|c|}{122.851} & \multicolumn{2}{|c|}{239.118} \\
\hline & $\mathrm{t}$ & Sig. & $\mathrm{t}$ & Sig. & $\mathrm{t}$ & Sig. & $\mathrm{t}$ & Sig. \\
\hline (Constant) & -5.888 & $0.000^{* *}$ & 2.628 & $0.009 * *$ & 11.053 & $0.000 * *$ & 6.312 & $0.000 * *$ \\
\hline LN_FL & 30.935 & $0.000^{* *}$ & 2.713 & $0.007 * *$ & 6.230 & $0.000 * *$ & 11.817 & $0.000 * *$ \\
\hline LN_D & 6.152 & 0.000 ** & -2.324 & $0.020^{*}$ & -10.830 & $0.000 * *$ & -6.157 & $0.000 * *$ \\
\hline DU_N & 7.074 & 0.000 ** & 11.791 & 0.000 ** & 4.260 & $0.000 * *$ & 7.047 & $0.000 * *$ \\
\hline DU_D & 25.115 & $0.000^{* *}$ & 27.623 & $0.000^{* *}$ & 21.898 & $0.000 * *$ & 6.996 & $0.000 * *$ \\
\hline DU_SE & 16.377 & $0.000^{* *}$ & 17.015 & $0.000^{* *}$ & 15.385 & $0.000 * *$ & 3.922 & $0.000 * *$ \\
\hline DU_T & 9.051 & $0.000^{* *}$ & 8.906 & $0.000^{* *}$ & 9.625 & $0.000 * *$ & 0.050 & 0.960 \\
\hline DU_TE & 17.499 & $0.000^{* *}$ & 2.404 & $0.016^{*}$ & -0.532 & 0.595 & 10.065 & $0.000 * *$ \\
\hline DU_CL & 14.986 & $0.000^{* *}$ & 3.887 & $0.000^{* *}$ & 6.586 & $0.000 * *$ & 8.477 & $0.000 * *$ \\
\hline DU_PS & 24.783 & $0.000^{* *}$ & 24.836 & $0.000^{* *}$ & 16.522 & $0.000 * *$ & 20.797 & $0.000 * *$ \\
\hline DU_CC & 3.495 & $0.000 * *$ & $0.893 *$ & $0.372 * *$ & $0.015^{*}$ & 0.988 & 5.650 & $0.000 * *$ \\
\hline DU_TT & 11.279 & $0.000^{* *}$ & 12.049 & $0.000^{* *}$ & 7.974 & $0.000 * *$ & 15.437 & $0.000 * *$ \\
\hline Y2001 & 6.740 & $0.000^{* *}$ & 6.571 & $0.000^{* *}$ & 1.582 & 0.114 & 4.830 & $0.000 * *$ \\
\hline Y2002 & 19.073 & $0.000^{* *}$ & 14.131 & $0.000^{* *}$ & 8.776 & $0.000 * *$ & 10.474 & $0.000 * *$ \\
\hline Y2003 & 29.827 & $0.000^{* *}$ & 25.560 & $0.000^{* *}$ & 18.844 & $0.000 * *$ & 19.050 & $0.000 * *$ \\
\hline Y2004 & 40.701 & $0.000^{* *}$ & 34.363 & $0.000^{* *}$ & 25.168 & $0.000 * *$ & 21.586 & $0.000 * *$ \\
\hline Y2005 & 43.779 & $0.000^{* *}$ & 37.298 & $0.000^{* *}$ & 29.104 & $0.000 * *$ & 24.638 & $0.000 * *$ \\
\hline Y2006 & 48.185 & $0.000^{* *}$ & 40.963 & $0.000^{* *}$ & 30.417 & $0.000 * *$ & 27.323 & $0.000 * *$ \\
\hline Y2007 & 49.768 & $0.000^{* *}$ & 42.097 & $0.000^{* *}$ & 32.201 & $0.000^{* *}$ & 29.498 & $0.000 * *$ \\
\hline Y2008 & 19.566 & $0.000^{* *}$ & 16.108 & $0.000^{* *}$ & 14.880 & $0.000 * *$ & 10.981 & $0.000 * *$ \\
\hline CH_R7000M & -9.791 & $0.000^{* *}$ & -4.311 & $0.000^{* *}$ & 4.068 & $0.000^{* *}$ & $-0.717^{*}$ & 0.473 \\
\hline INT_R7000M & 16.276 & $0.000^{* *}$ & 7.041 & $0.000^{* *}$ & 1.998 & $0.046 *$ & 8.717 & $0.000^{* *}$ \\
\hline NSV & \multicolumn{2}{|c|}{21} & \multicolumn{2}{|c|}{20} & \multicolumn{2}{|c|}{19} & \multicolumn{2}{|c|}{19} \\
\hline
\end{tabular}

$T_{0.05}=1.96 \mathrm{p}<0.05, * * T_{0.01}=2.576 \mathrm{P}<0.01$

$\mathrm{NSV}=$ number of significant variable

Table 5.9 Chow test results of optimal urban configuration specification

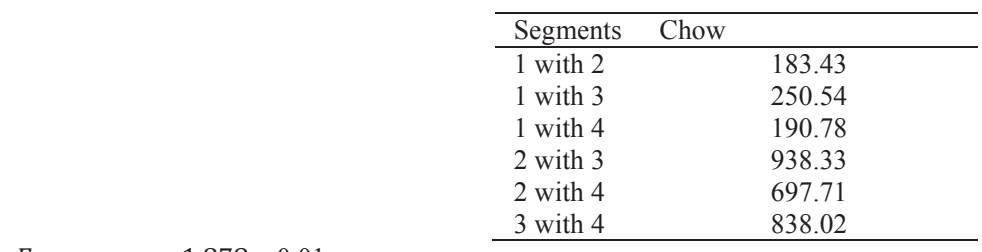




\subsubsection{Nested urban configuration and dwelling type specification}

The second way to specify housing submarket is to nest both dwelling types and urban configuration features at radii $7 \mathrm{~km}$. The reasons of justification for this approach are two folds: firstly, compared with traditional specification only by dwelling types, I expect considering the all urban configurational features could improve the estimation. Furthermore, I also expect to compare the performance of specifications by dwelling types within the pre-defined geographical area and new alternative spatial areas. The two-step clustering analysis results are showed in table (5.10), although the ratio of distance of both four and five clusters are higher than others, five clusters has the smallest ratio of BIC change, thus, the optimal numbers of submarket specified by nesting both dwelling types and urban configuration features is five.

Figure (5.3) shows the spatial pattern of the five housing submarkets. It is found that building structure characteristics (e.g. detached, semidetached, terrace and flat) have the strongest influence on the five housing submarkets, and each building type is associated with a different combinations of high and low values of integration and choice. For example, the submarket of flats is associated with high street connections and high traffic flow, and detached and semidetached houses require similar urban configuration features with lowest integration and higher choice values. Furthermore, it is noted that the largest portion of building type is terraced housing, and it is divided into two groups which are associated with high and low space syntax integration and choice. Two thirds of terrace houses are in cluster two and are located in places with the highest accessibility and highest traffic volume whereas the rest of the terraced housing are located in cluster three with street segments less connected and lower levels of traffic volume.

By examining the social economic status of the residents in each submarket, it is possible to state that different demands from different classes cause housing market segmentation. For example, as expected, the largest group of people who choose flats are from the "city living" group. More than half of the people who choose detached housing are from the group "prospering suburbs", and "prospering suburbs" and 
"typical traits" take up nearly two thirds of the people choosing semi-detached housing. In comparison, the other terraced-housing submarket, which has higher choice and integration value, is mainly occupied by the city living cohort and people of similar socioeconomic characteristics such as blue collars group,. This in turn implies that the location of this submarket is near the city center. With regards to terrace houses that have different configurational features, the proportion of different socio-economic classes are slightly different. For example, it is found that more people from the "blue collar" group and less "city living" prefer terraced housing located in urban suburb areas, which have lower street segment connectivity and traffic flow.

Table 5.10 Cluster results of nested urban configuration and building type specification

\begin{tabular}{|c|c|c|c|c|}
\hline \multicolumn{5}{|c|}{ Auto-Clustering } \\
\hline Number of Clusters & $\begin{array}{l}\text { Schwarz's Bayesian } \\
\text { Criterion (BIC) }\end{array}$ & BIC Changea & $\begin{array}{l}\text { Ratio of BIC } \\
\text { Changesb }\end{array}$ & $\begin{array}{c}\text { Ratio of } \\
\text { Distance } \\
\text { Measuresc }\end{array}$ \\
\hline 1 & 92234.001 & & & \\
\hline 2 & 54172.863 & -38061.138 & 1.000 & 2.063 \\
\hline 3 & 35766.199 & -18406.664 & 0.484 & 1.477 \\
\hline 4 & 23328.847 & -12437.352 & 0.327 & 2.319 \\
\hline 5 & 18010.103 & -5318.744 & 0.140 & 2.268 \\
\hline 6 & 15708.879 & -2301.224 & 0.060 & 1.017 \\
\hline 7 & 13448.247 & -2260.632 & 0.059 & 1.312 \\
\hline 8 & 11743.573 & -1704.674 & 0.045 & 1.429 \\
\hline 9 & 10573.904 & -1169.670 & 0.031 & 1.216 \\
\hline 10 & 9625.946 & -947.958 & 0.025 & 1.006 \\
\hline 11 & 8683.699 & -942.247 & 0.025 & 1.058 \\
\hline 12 & 7797.218 & -886.480 & 0.023 & 1.484 \\
\hline 13 & 7225.345 & -571.874 & 0.015 & 1.309 \\
\hline 14 & 6806.877 & -418.467 & 0.011 & 1.132 \\
\hline 15 & 6446.276 & -360.602 & 0.009 & 1.149 \\
\hline
\end{tabular}




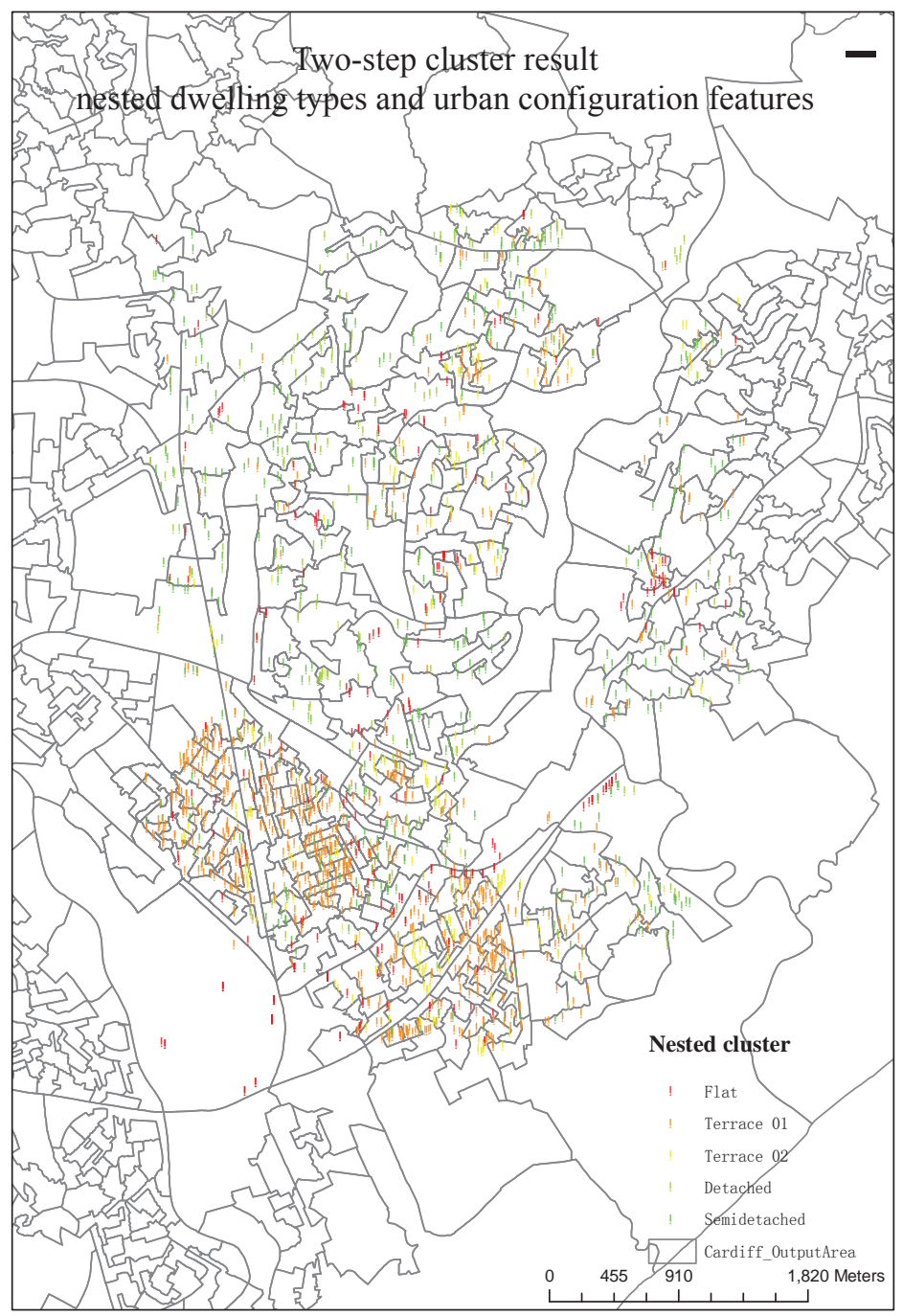

Figure 5.3 Two-step cluster result of nested dwelling type and all urban configurational features Source: author 


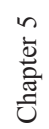

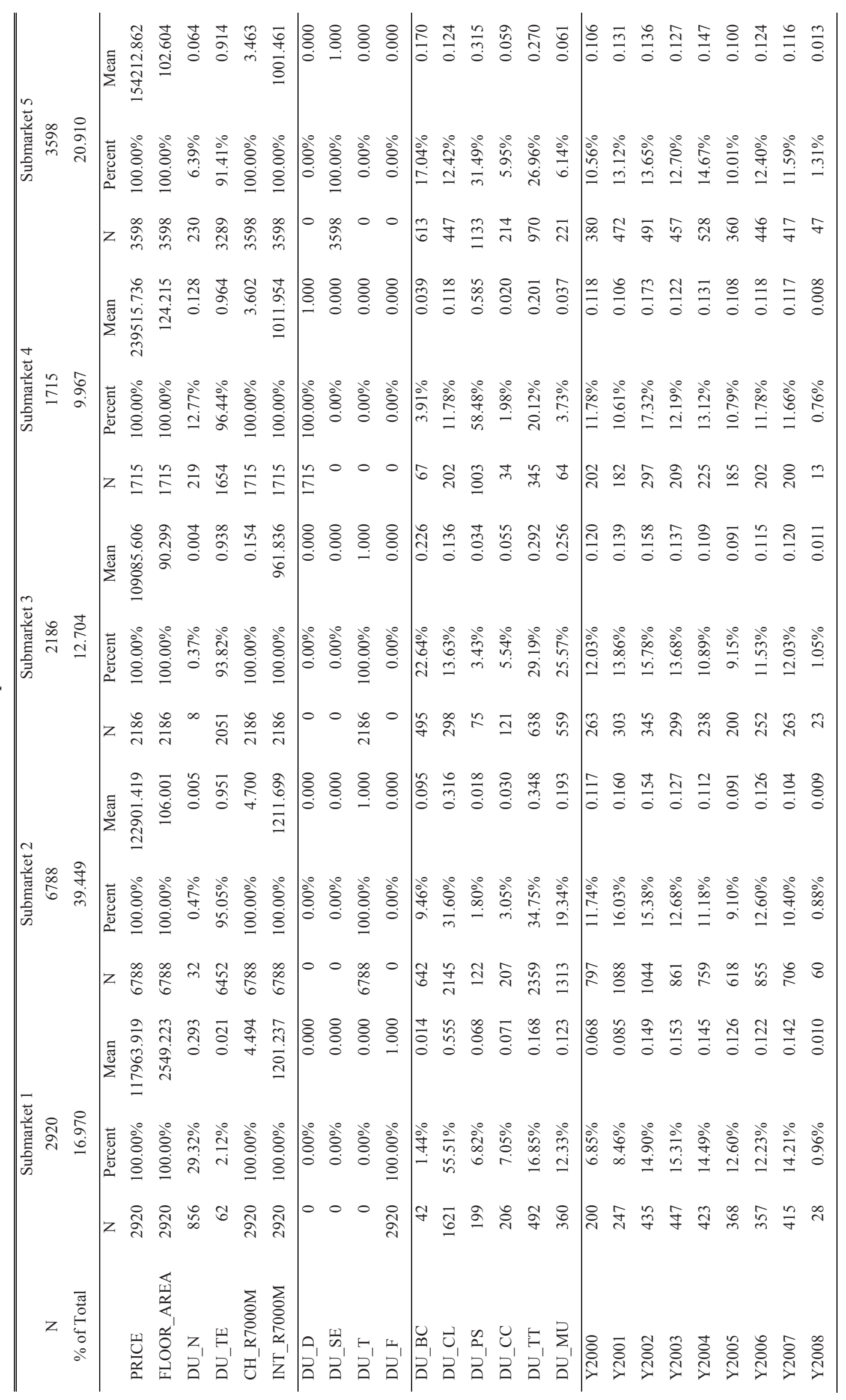


Table (5.12) shows the results of five submarkets estimation specified by nested dwelling types and all urban configuration features. Key findings are highlighted as follows. Firstly, all models of the five submarkets have good results, since all F tests are significant and the adjusted R-square is within the range of 0.56 to 0.65 . Secondly, the location characteristic of distance to CBD is significant with a negative sign and found in the submarket of urban suburb area, where both "integration" and "choice" are not high. Thirdly, the sign of urban configuration is constant, but it is found that urban configurational features do not impact the submarket of detached houses, as both integration and choice at $7 \mathrm{~km}$ radii are insignificant at 5\% level. Furthermore, choice is also found insignificant in the terraced submarket in suburban areas, as the variance of traffic flow is much lower in suburbs than centrally. This indicates that comparing inner-city and outer-city, when people choose their properties, they do not care about the traffic flow in the outer-city. Fourthly, it is seen that people "constrained by circumstances" are more sensitive to the submarkets associated with high value of choice, as the variable is insignificant in both submarket two and three. The results of the Chow tests for structural change in pricing models are presented in table (5.13). All F tests are significant at 0.01 level of confidence, showing that there is strong evidence to support the existence of five submarkets using this method.

Table 5.12 Estimation results of nested all urban configurational features and building type specification

\begin{tabular}{|c|c|c|c|c|c|c|c|c|c|c|}
\hline & \multicolumn{2}{|c|}{ Submarket 1} & \multicolumn{2}{|c|}{ Submarket 2} & \multicolumn{2}{|c|}{ Submarket 3} & \multicolumn{2}{|c|}{ Submarket 4} & \multicolumn{2}{|c|}{ Submarket 5} \\
\hline $\mathrm{N}$ & \multicolumn{2}{|c|}{2920} & \multicolumn{2}{|c|}{6788} & \multicolumn{2}{|c|}{2186} & \multicolumn{2}{|c|}{1715} & \multicolumn{2}{|c|}{3598} \\
\hline Adjust R-square & \multicolumn{2}{|c|}{0.638} & \multicolumn{2}{|c|}{0.565} & \multicolumn{2}{|c|}{0.628} & \multicolumn{2}{|c|}{0.591} & \multicolumn{2}{|c|}{0.654} \\
\hline F test & \multicolumn{2}{|c|}{272.082} & \multicolumn{2}{|c|}{474.976} & \multicolumn{2}{|c|}{200.454} & \multicolumn{2}{|c|}{115.747} & \multicolumn{2}{|c|}{358.349} \\
\hline \multirow[t]{2}{*}{ RSS } & \multicolumn{2}{|c|}{315.488} & \multicolumn{2}{|c|}{736.618} & \multicolumn{2}{|c|}{207.866} & \multicolumn{2}{|c|}{186.696} & \multicolumn{2}{|c|}{320.484} \\
\hline & $\mathrm{t}$ & Sig. & $\mathrm{t}$ & Sig. & $\mathrm{t}$ & Sig. & $\mathrm{t}$ & Sig. & $\mathrm{t}$ & Sig. \\
\hline (Constant) & 1.238 & 0.216 & -4.338 & $0.000^{* *}$ & 6.783 & $0.000 * *$ & 2.352 & $0.019 *$ & 6.849 & $0.000 * *$ \\
\hline LN_FL & 14.430 & 0.000 ** & 22.954 & $0.000 * *$ & 8.843 & 0.000 ** & 23.663 & $0.000 * *$ & 21.904 & $0.000 * *$ \\
\hline LN_D & $-1.046^{*}$ & 0.296 & 4.600 & $0.000 * *$ & -6.577 & 0.000 ** & -2.248 & $0.025^{*}$ & -6.590 & $0.000 * *$ \\
\hline DU_N & 15.455 & $0.000 * *$ & 2.348 & $0.019 *$ & $1.235^{*}$ & 0.217 & 2.126 & $0.034 *$ & 9.951 & $0.000 * *$ \\
\hline DU_TE & 5.171 & $0.000 * *$ & 13.480 & $0.000^{* *}$ & 3.841 & $0.000 * *$ & 13.389 & $0.000 * *$ & 8.304 & $0.000 * *$ \\
\hline
\end{tabular}




\begin{tabular}{|c|c|c|c|c|c|c|c|c|c|c|}
\hline DU_CL & 6.629 & $0.000 * *$ & 11.257 & $0.000 * *$ & 4.844 & $0.000 * *$ & 5.479 & $0.000 * *$ & 8.639 & $0.000 * *$ \\
\hline DU_PS & 19.342 & $0.000 * *$ & 9.145 & $0.000 * *$ & 8.521 & $0.000 * *$ & 15.323 & $0.000 * *$ & 26.534 & $0.000 * *$ \\
\hline DU_CC & 1.177 & 0.239 & 3.051 & $0.002 * *$ & 1.111 & 0.267 & 2.281 & $0.023 *$ & 3.560 & $0.000 * *$ \\
\hline DU_TT & 5.558 & $0.000 * *$ & 9.694 & $0.000 * *$ & 14.916 & $0.000 * *$ & 6.028 & $0.000 * *$ & 13.156 & $0.000 * *$ \\
\hline Y2001 & 4.226 & $0.000 * *$ & 7.543 & $0.000 * *$ & 3.848 & $0.000 * *$ & 3.140 & $0.002 * *$ & 5.067 & $0.000 * *$ \\
\hline Y2002 & 11.886 & $0.000 * *$ & 19.474 & $0.000 * *$ & 9.382 & $0.000 * *$ & 5.949 & $0.000 * *$ & 13.545 & $0.000 * *$ \\
\hline Y2003 & 16.896 & $0.000 * *$ & 33.003 & $0.000 * *$ & 20.076 & $0.000 * *$ & 10.066 & $0.000 * *$ & 22.406 & $0.000 * *$ \\
\hline Y2004 & 23.565 & $0.000 * *$ & 41.305 & $0.000 * *$ & 25.291 & $0.000 * *$ & 13.389 & $0.000 * *$ & 29.451 & $0.000 * *$ \\
\hline Y2005 & 27.309 & $0.000 * *$ & 43.285 & $0.000 * *$ & 26.776 & $0.000 * *$ & 16.823 & $0.000 * *$ & 31.807 & $0.000 * *$ \\
\hline Y2006 & 28.280 & $0.000 * *$ & 48.603 & $0.000 * *$ & 30.735 & $0.000 * *$ & 17.075 & $0.000 * *$ & 34.536 & $0.000 * *$ \\
\hline Y2007 & 29.400 & $0.000 * *$ & 49.810 & $0.000 * *$ & 32.361 & $0.000 * *$ & 19.014 & $0.000 * *$ & 35.497 & $0.000 * *$ \\
\hline Y2008 & 11.116 & $0.000 * *$ & 19.396 & $0.000 * *$ & 14.398 & $0.000 * *$ & 6.418 & $0.000 * *$ & 15.606 & $0.000 * *$ \\
\hline CH_R7000M & -7.366 & $0.000 * *$ & -9.057 & $0.000 * *$ & -1.329 & 0.184 & 1.532 & 0.126 & -3.174 & $0.002 * *$ \\
\hline INT_R7000M & 11.917 & $0.000 * *$ & 19.618 & $0.000 * *$ & 6.499 & $0.000 * *$ & 1.594 & 0.111 & 9.957 & $0.000 * *$ \\
\hline NSV & \multicolumn{2}{|c|}{16} & \multicolumn{2}{|c|}{18} & \multicolumn{2}{|c|}{15} & \multicolumn{2}{|c|}{16} & 18 & \\
\hline
\end{tabular}

$T_{0.05}=1.96 \mathrm{p}<0.05, * * T_{0.01}=2.576 \mathrm{P}<0.01$

$\mathrm{NSV}=$ number of significant variable

Table 5.13 Chow test results of nested all urban configurational features and building type specification

\begin{tabular}{ll}
\hline Segments & Chow \\
\hline Submarket 1 with 2 & $420.83^{* *}$ \\
Submarket 1 with3 & $715.49^{* *}$ \\
Submarket 1 with4 & $651.10^{* *}$ \\
Submarket 1 with5 & $679.83^{* *}$ \\
Submarket 2 with3 & $481.56^{* *}$ \\
Submarket 2 with4 & $462.38^{* *}$ \\
Submarket 2 with5 & $437.88^{* *}$ \\
Submarket 3 with4 & $727.69^{* *}$ \\
Submarket 3 with5 & $774.56^{* *}$ \\
Submarket 4 with5 & $714.70^{* *}$ \\
\hline
\end{tabular}

$F_{(20, \mathrm{n}-2 \mathrm{k})}=1.878 \mathrm{a}=0.01$

\subsubsection{Estimation of weighed standard error}


Table 5.14Estimation results of weighed standard error

\begin{tabular}{llc}
\hline \hline Stratification scheme & Standard error & $\%$ reduction \\
\hline Market-wide model & 1942.597 \\
Structure definition identified by dwelling type & 642.615 & $66.92 \%$ \\
Nested definition identified by dwelling-type segments in north and south & 394.090 & $79.71 \%$ \\
Submarket specified only by urban configuration at 7000M radius & 744.060 & $61.70 \%$ \\
Submarket specified by both urban configuration and dwelling types at 7000M radii & 456.726 & $76.49 \%$ \\
\hline
\end{tabular}

Finally, i use the weighted standard test applied by Schnare and Struyk(1976) to evaluate the improvement of segmented market models in terms of standard error estimation. For the weighted standard error test, there is no strict requirement on the size of the low threshold of evidence, or significance and overall variability in housing prices. Schnare and Struyk accepted this threshold as 10 percent in their studies whereas Dale and Johnson (1982) suggested five percent. The two new alternative methods and the two traditional methods defining the housing submarket all pass the weighted standard error test, even at the strictest level, as shown in table (5.14).

From that table (5.14), it is possible to see that the method specifying submarkets using nested spatial indicator and structure with prior experience has the best results, which has improved the market-wide estimation by $79.71 \%$. The second best performance specification is defining submarkets by both urban configuration and dwelling types at $7000 \mathrm{~m}$ radii, which has improved the error rate by $76.49 \%$. The third best is the structure definition by dwelling type.

Although the new method identifying submarkets only by urban configuration features at radii $7 \mathrm{~km}$, is not the best, the model has still improved the estimation by $61.70 \%$. The spatial and structure nested method has the best performance overall since it is an ad hoc method. The urban configuration and dwelling types nested identification is slightly weaker than the traditional spatial and structure nested model, but the whole process can be deemed more objective. As Orford (2000) highlighted, 
the building structure features are important for housing submarkets in Cardiff housing market, which means that the housing market in Cardiff can be segmented according to its building structure features. In this case, each building type group reflects the price unit of each characteristic and people's willingness to pay is constant. However, the results of clustering nested building types and urban configurational features shows that each building type is associated with specific combination of high and low integration and choice values, which suggests that the building structure features interact with urban configurational features in complex ways. Furthermore, the weighed standard error test shows the improvements of specification by building type and the optimal urban configurational features are quite close with a difference of only $5.22 \%$.

On the basis of these results, it may be suggested that for housing markets where the dwelling type is uniform, urban configurational features could be an alternative specification method for submarkets, since I have shown that there is heterogeneous demand within the housing market as a whole for different kinds and levels of network connectivity and conversely, and different classes of people have homogeneous demand for urban configurational features of housing property. Cardiff proves to be an interesting location for this experiment since it has become obvious that the strong distinctions made in the market between building-types, means that this becomes a dominant dimension in creating sub-markets. In spite of this, network configuration measured by space syntax still adds discriminating power in explaining differences in clusters of house prices. I can be sure therefore that in markets with far more homogenous housing stock such as Chinese cities, urban configuration is likely to be an important determinant in sub-market formation.

\subsection{Summary}

This study supports the large amount of researches into the means of identifying 
housing submarkets that have found that building structure features are important inreflecting people's homogenous perceptions towards property attributes. However, it has added new knowledge designed to address the lack of understanding about how to deal with submarkets when the dwelling type is homogenous, for example uniformly simplex as in many Chinese cities. I have shown that configurational data recovered from a graph model of a street network adds significant power in discriminating between housing sub-markets and thus provides a potentially fruitful approach to analyzing sub-markets in China.

Although a non-spatial specification of sub-markets can to a degree relieve some of the problems of traditional ad hoc geographical sub-market specification these methods require social neighborhood characteristics to be constant over time and such methods are thus ill-fitted to rapidly transforming countries. The objective of this study has therefore been to test whether urban configurational features can offer a new alternative way to specify housing submarkets, since network configuration is likely to be more stable than other neighborhood characteristics. The accessibility metrics derived from space syntax models of housing areas would intuitively seem to be important contributors to locational value. In order to verify the assertion, I have compared the model performances of network-based sub-market specifications against traditional means. Two alternative methods were specified: one used only optimally measured urban configurational features, and the other used nested optimal urban configurational feature combined with dwelling types.

Two-step clustering analysis found that both building types and social economic classes are associated with the indicators of space syntax namely integration and choice. For example, flats require street segments with high levels of street connectivity and high traffic flow whereas detached and semidetached houses require similar urban configuration where street connection is poor but with high levels of traffic flow. Furthermore, people of middle class cohorts or above tend to live further 
away from the city center since they have access to cars and consequently are less sensitive to how well street segments are connected. This is in stark contrast to lower income people who are sensitive to the geometrical pattern of street segments.

Finally, the weighted standard error tests show that all specifications using space syntax metrics have significantly improved the estimation over and above the market-wide model. Although the specifications based on urban configurational features are not the best performing models, these results are quite close to the traditional specifications and they are (a) more objective in their approach and (b) less reliant on a housing market culture that has evolved around distinctly differentiated building types. Therefore, it seems justifiable to conclude that a housing sub-market model based on urban configurational features can be an alternative way to deal with cases where the building type is uniformly simplex and where there are low levels of observations of social neighborhood preferences. 


\section{Chapter Six:}

\section{Identifying the micro-dynamic effects of urban street configuration on house price volatility using a panel model}

\subsection{Introduction}

For the majority of households, housing represents the main component of wealth, and the "wealth effect" of housing on consumption is significant (Case et al. 2005). Furthermore, the housing market impacts the economy not only through wealth effects, but also has influences on other markets (e.g. the mortgage market, mortgage insurance, mortgage backed bonds, and consumer durables). Therefore, understanding the sources of housing market price volatility has great significance or the wider economy and the welfare of society. Despite the importance of the housing market, most empirical analyses mainly focus on exploring the macro determinants of house price movements over time using aggregated data (e.g. real income, real GDP, tax rates, interest rates, population, construction costs and consumer price index) (Holly and Jones 1997; Hort 1998; Takala and Barot 1998; Meese and Wallace 2003; Harter-Dreiman 2004; Riddel 2004). At the micro level, there are also researchers who identify the price impact of one-time changes of building characteristic, measuring the added value for housing price over time by hybrid repeat sales models and hedonic models (McMillen 2003; Case et al. 2006; McMillen and Thorsnes 2006; Tian 2006; Noonan 2007). However, hybrid repeat sales and hedonic models have been criticized for the basis of sample selection and underestimation (Polinsky and Rubinfeld 1977; Polinsky and Shavell 1982). Recently, Iacono and Levinson (2011) examined the micro dynamic relationship between accessibility and land value over 
time by first difference estimation. They used property sales data of Minneapolis-St. Paul region for the years 2000 and 2005, and measured of accessibility combined with regional accessibility data for their respective years to predict changes in prices over time in a particular location. However, they failed to find any significant micro dynamic effects of accessibility on land value over time. They also noted that measuring changes in accessibility should be described more accurately than they were able to. Although these approaches could address the problem of omitted variable, they do not consider the unobserved heterogeneity variation over time (e.g. housing policy change and construction cost change), and assume the macro factors to be constant, which also may lead to estimation bias.

However, few studies have examined geographical determinants of house price volatility within a region or city scale, although many scholars assert that the value of accessibility could lead to changes in housing price dynamics (Capozza and Helsley 1989). Currently, China is undergoing rapid the urbanization, and with respect to its scale, it is perhaps the largest the world has ever experienced (Zhang and Song 2003). As part of this process, there have been numerous investments into road network developments situated on the urban fringe in order to cope with the rapid expansion of cities and its populations (Zhang and Zhao 2009). In most Chinese cities, it is easy to observe how rapid urban spatial expansion can impact on the dynamic change of housing price in the local housing market, especially after 2003. Many house purchasers found that the price of properties located near existing or future transport hubs are more likely to increase. Consequently, housing buyers tend to have a clear belief that infrastructure improvement projects will add further value to their property.

Against this background, the study readdresses the hypothesis that there is a micro-dynamic relationship between urban morphology and housing price, using a large panel dataset for city of Nanjing, China from 2005 to 2010.Three main questions are answered in this study. Firstly, is there a micro-dynamic relationship 
between urban morphology and housing price? Secondly, do all street network improvements produce a positive spillover effect captured in property value; and if not then what kind of street network improvement and at what scale are such improvements good for the house owner? Thirdly, I ask whether this relationship is dynamic synchronously over both space and time, and whether submarkets exist as a result of accessibility patterns.

Compared with previous studies, several points should be made. Firstly, the study applies the average selling price rather than the transaction price from survey data. The reasons for this are explained at a later point of this chapter. Secondly, a location-based social network analysis method, known as space syntax, is employed in this study to capture the accessibility changes created by street network improvements over time. Thirdly, I ran a random intercept model instead of alternatives such as a first difference model, hybrid repeat sale model and hedonic model, as during the period in which the data was gathered, housing policy governing the regulation of multiple properties changed several times, thus the unobserved heterogeneity could be absorbed by the intercept in a fixed effect model. Furthermore, the assumptions of a first difference model are less restrictive than the assumptions of weak exogeneity required for an unbiased fixed effects (FE) estimator (i.e. homoscedasticity and no serial correlation in error term). A fixed effects model is efficient under independence of error term and a first difference model is efficient under perfect serial correlation in the error term (Mendelsohn et al. 1992).

The structure of this chapter is organized as follows: in section two, I review studies that examine housing price volatility both at macro and micro level. In section three, space syntax network analysis and longitudinal random intercept models are explained and illustrated. Section four introduces the study area and survey data, and results are presented in Section five, utilizing five different models (e.g. pooled model, individual-fixed effects model, random effects model, time-fixed effect model and 
two-way effects model). Insights from the empirical results are discussed in section 6; and Section 7 summarizes the findings and concludes.

\subsection{Literature review}

\subsubsection{Cross-sectional static house price models}

Urban researchers have long recognized the limitations of the monocentric concept on several levels (e.g. polycentric employment, assumption of exogenous employment location) (e.g. Boarnet 1994). As an alternative, the notion of accessibility is taken by most researchers exploring the locational determinants of house price following Rosen's (1974b) hedonic price model based on cross-section data. Some researchers have found that accessibility in terms of easy access to different services (such as rail station) adds value to properties. For example, Dewees (1976) explored the rail travel costs' impact on residential property values, and he found that a subway station increases the site rent with a radii of one third mile. In a similar study, Bowes and Ihlanfeldt (2001) found that rail stations with parking facilities have a higher positive impact on housing price, but its impact power still depends on how proximity to the CBD. In fact, the most megacity shows a tendency of ploycentric in spatial, such as Osland and Thorsen (2008)explored the effect of urban attraction and labor-market accessibility on housing price by utilizing a gravity-based labor-market accessibility. They found that labor-market accessibility is not an adequate alternative to distance from the CBD; illustrating that there are multiple attributes bundled into the concepts of central accessibility. Recently, Adair et al. (2000)focused on the relationship between accessibility, price, and location of owner-occupied housing in the Belfast urban area, and they adopted a traffic gravity model, calculating the accessibility index of locations to various opportunities by different types of vehicle. They find that the impact of accessibility is not consistent via space as it is of little significance at a city-wide scale, but become significant at a sub-market level, in particularly in 
lower-income areas.

\subsubsection{Hybrid repeat sales model with hedonic model}

However, most hedonic models rely on cross-sectional data, and suffer from a number of well-known problems (e.g. omitting variables). Besides this, housing quality and other characteristics can change profoundly over time. McConnell and Walls (2005) stated thatthe standard cross-sectional approach precludes a dynamic perspective on house price formation. McMillen (2003) also discussed the limitations of static hedonic models, noting that the unit price of housing can only be estimated accurately from a simple hedonic house value function if data are available on all housing characteristics.

Therefore, the repeat sale model is often employed for house price index construction without hedonic characteristics, as it assumes that the coefficients of each characteristic are constant over time. Some researchers have combined hedonic model and repeat sales models, into a hybrid model to identify the one-time changes of one characteristic, adding the value for house price over time (Case et al. 2006). For example, McMillen (2003) used an alternative repeat sales model to identify changes in house price distance gradients in Chicago. He employed transaction data for Chicago from January 1, 1983 to December 31, 1998, and found that house prices in the City were not affected significantly by distance from the CBD in the early to mid 1980s. Furthermore, McMillen and McDonald (2004) examined the effect of the new rapid transit line on single-family house price, in particular investigate the impact of before and after the opening of the line from downtown Chicago to Midway Airport . The results showed that since opening of the line, the house price gradient with respect to distance to the station having been $-4.2 \%$ per mile before 1987 and $-19.4 \%$ during 1991-1996. They also note that the repeat-sales method yields an identical result for this change in the price gradient. McMillen (2004) measures the effect of 
airport expansion on property value around Chicago O'Hare. He applied a simple hedonic regression separately for 1997 and 2000, with the results suggesting that aircraft are becoming so much quieter, and its negative externality is almost insignificant, which indicates that the airport could be expanded without negatively affecting the local housing price.

Tian (2006) examined the impact of two transport projects on residential property values in Guangzhou, China. Calculating a general housing index through repeat-sales and hedonic models, she found that the construction of Metro line 2 has a positive impact on the value of residential property within walking distance of a station. However, within a pre-defined area around the inner ring road, the repeat-sales model found the construction of the Inner Ring Road project to have a negative impact on house price. Case et al. (2006) attempted to apply a hybrid repeat-sales and hedonic model to explore the impact of environmental contamination on condominium price using a dataset for Scottsdale, Arizona. From the results, they found that the environmental contamination has a negative impact on local prices but that the impact does not appear until several years after the contamination became publicly known. What is more, they argue that it is necessary to incorporating hedonic characteristics into repeat-sale models since the hedonic model assumes all characteristics are fixed over time, but the parameters of location variables can vary profoundly over time by urban growth and / or development.

Noonan (2007) examined the effect of historic landmarks on property value in Chicago with a hybrid hedonic and repeat-sales model. Firstly, he applied a hedonic price analysis for the observations in the affect area, and he noted the hedonic price model omitted some variable, which could lead to the results bias. Secondly, a repeat-sales approach demonstrated significant spillover effects of landmark designation on prices. Unlike other studies, Noonan considered sample selection bias and the issues of spatial autocorrelation. 
It is clear from this review that researchers favour applying a hybrid repeat sales and hedonic modeling approach, examining whether one-time changes of characteristic add the value for housing price over time within a pre-defined area. There are, however, two major problems for the hybrid repeat sales and hedonic model that are pointed out in the literature: (i) there is sample selection bias because houses traded in repeat sales data sets may well have special characteristics, meaning that model parameters are not generalizable to the whole housing markets(Clapp and Giaccotto 1992). Due to certain characteristics, some dwelling types transact more often than others, and some do not transact with normal frequency or at all. (ii) The theoretical models of Polinsky and Rubinfeld (1977) and Polinsky and Shave (1982) show that the repeat sale approach can work if the affected area can be thought of as an "open city", which means that the affected area is a small part of the housing market. If the pollution effect does reduce housing prices across an entire market, the repeat sale approach will underestimate pollution damage.

\subsubsection{Panel models}

Panel models are distinguished from repeat sales models by the monitoring of prices for all houses in the study with both spatial and intertemporal factors, and it is particularly appropriate for measuring urban morphology effects on residential property values. First, the analysis of the repeat sales of specific properties allows very careful control of the unwanted effects of house-to-house differences. Second, intertemporal pattern change in urban morphology is easy to measure, so that one can compare before and after effects. These intertemporal effects can be as powerful as the cross sectional effects, measuring the effect of urban street layout change. Third, urban morphology dynamic effects tend to be at a city scale rather in a pre-defined affected area. As Hsiao (2003) and Klevmarken (1989) point out, the most important benefit from using panel data is that panel data are able to control individual 
heterogeneity over space and time, whereas non-panel time-series or cross-section studies cannot. Panel models allow researchers to analyze macro-dynamic and micro-dynamic effects that cannot be addressed using non-panel datasets. In addition, panel models could increase the degrees of freedom and reduce collinearity among explanatory variables -hence improving the efficiency of econometric estimates.

Empirical studies of the housing market distinguish two main types of drivers: macroeconomic and micro geographical drivers. Recently, an increasing number of studies have examined the macro determinants of house price movements over time by aggregated data across countries (e.g. real income, real GDP, tax rates, interest rates, population, construction costs and consumer price-index). For example, Apergis and Rezetis (2003) examined the dynamic effects of macroeconomic factors on housing price, and he found that mortgage rate had the highest explanatory power in the variation of housing price, and inflation and employment had the second and third highest power. Kholdy and Sohrabian (1998) attempted to explain housing cyclesfrom1986 to 1994 in California. The results of the study suggest that speculative behavior and expectations of capital gain played a stronger role than economic fundamentals to explain housing cycles. Stern (1992) examined the determinants of house price inflation in UK for the period 1971to 1989. He found that real income was the most important determinant of house price inflation; housing supply had a somewhat greater effect on prices than had interest rates. Stevenson (2004) presented strong evidence that housing and inflation are cointegrated in the long run by utilizing cointegration tests. Conventional ordinary least squares (OLS) models provided less conclusive results. Jud and Winkler (2002) also showed that changes in real income, construction costs and interest rates, as well as growth of population are significant factors for real U.S. housing price appreciation.

However, few studies have examined the role of micro geographical determinants of house price volatility within a region or city, especially for geographical determinants. 
Capozza et al. (1986) confirm there is a significant positive relationship between urban growth and the housing price. Capozza and Helsley (1989) show in a dynamic model that the price of urban land has four additive components, which including the agricultural land rent, the cost of conversion, accessibility, and expected future rent increases. Urban development is an incremental process, where densities depend solely on economic conditions at the time of development. Densities may nevertheless decline with distance, because economic conditions change over time in particular ways (incomes increase or transportation costs fall), but within the urban area, land prices increase by the capitalized value of accessibility as distance to the CBD decreases.

Van de Vlist et al. (2011) address the interplay between demographic and housing market dynamics using the case of Haifa, Israel with 34 tracts over11 years. Their empirical analysis creates a house price index by tract and year to explore how demographic shocks and associated changes in housing demand affect the housing market. The results indicate house prices vary across submarkets in response to a demographic shock, and house prices in Haifa tend to converge across submarket with lower-end submarkets ending up with higher house prices than higher submarkets.

Mendelsohn et al. (1992) applies panel models to measure the disamenity from hazardous wastes on residential property by comparing a first differencing model and a fixed effect model. The panel models detect a significant reduction in housing values associated with the timing and location of the waste site area. Affected properties were estimated to have fallen between $\$ 7000$ and $\$ 10,000$ (1989 dollars) in value as a result of their proximity to the hazardous wastes in nearby waters.

Recently, Iacono and Levinson (2011) examine the dynamic relationship between accessibility and land value over time using first difference estimation, and ask whether spatial-temporal changes of accessibility are associated with the changes in 
land value. They used property sales data of the Minneapolis-St. Paul region for the years 2000 and 2005, and measured of accessibility using regional accessibility data. However, they found only a very marginal and statistically insignificant effect of accessibility improvements on prices. They also note that measuring changes in accessibility and house prices over time should be more accurately treated in theory and empirical studies. This is the specific trigger for the study reported in this chapter.

To summarize, the number of studies examining the determinants of urban house price dynamics is limited, and few studies investigate the influence of geographical determinants on house prices dynamic, especially at a street level. The motivation of my research is to examine whether changes in the accessibility contained in an urban street network may be a determinant of the volatility of house prices at micro level in the short-run. I investigate this using data from 2005 to 2010 in Nanjing, China. It is well known that a variety of new town developments and street network improvement projects were initiated in both suburb and the metropolitan areas of Nanjing, in order to increase street connectivity and to relieve traffic pressure brought by rapid urbanization(Zhang and Zhao 2009).

The hypothesis is that improvements in street connectivity, lead to spillover effects on housing price. Or put another way: changes in street network over time, have the effect of redistributing a city's external economies by virtue of the fact that it is via the street network that individuals can transact with each other. Put yet another way, I hypothesize (a) that urban agglomeration economies are continually being redistributed spatially via incremental changes in the street network and (b) that these externality effects are capitalized in the housing market and are measurable via house prices. 


\subsection{Methodology}

\subsubsection{Space syntax method:}

The network measures used in this chapter have already been described already in Chapter three. Indices of both integration and choice are applied in this study. However, I specify experimentally the radii of $800 \mathrm{~m}, 1200 \mathrm{~m}, 2 \mathrm{~km}, 5 \mathrm{~km}, 8 \mathrm{~km}, 10 \mathrm{~km}$, $12 \mathrm{~km}, 15 \mathrm{~km}, 20 \mathrm{~km}$ and infinite distance as space syntax connectivity zones, capturing the urban configuration features.

\subsubsection{Panel model}

The basic linear panel models can be described as following general model:

$$
y_{i t}=\alpha_{i t}+\beta_{i t}^{T} x_{i t}+u_{i t} \quad(i=1, \ldots, N ; t=1, \ldots, T) \quad \text { Equation (6.1) }
$$

Where:

$i$ denotes households, individuals, firms, countries, etc.

$t$ denotes time.

$\alpha$ is the unknown intercept for each entity.

$\beta$ isK $\times 1$ and $x_{i t} \mathrm{t}$ is the $i t^{\text {th }}$ observation on $\mathrm{K}$ explanatory variables.

$u_{i t}$ isa random disturbance term of mean 0 .

The pooled model requires both intercept and parameters to be constant over time, which means that $\alpha_{i t}=\alpha$ for all, $t$ and $\beta_{i t}=\beta$ for all $i, t$. That is a standardlinear model for pooling data which acrossiand $t$.

If decompose the error term in two components, the model could be:

$$
u_{i t}=u_{i}+v_{i t}
$$


Where:

$u_{i}$ is the fixed effect or unobserved heterogeneity.

$v_{i t}$ isidiosyncratic error.

Note that if the dataset has observations for the same time periods for all individuals the approach is called a balanced panel. Otherwise, the data are an unbalanced panel data.

If only the intercept varies but parameters are constant over time, this is called a Fixed-effects model. It assumes that the error term has two separate components, one of which is a specific effect. The idiosyncratic error can vary over individuals and time, which contravene the strict assumptions for standard OLS error terms. We omit the constant, because it would be collinear with $u_{i}$.

$$
y_{i t}=\beta_{i t}^{T} x_{i t}+u_{i}+\varepsilon_{i t}
$$

There are three types of specific effect, namely individual, time, and two-ways. If the unobserved heterogeneity is constant over time, like culture, religion, gender, race, which do not vary over time, the individual-fixed effect model will be appropriate. Time-fixed effects models require the only unobserved heterogeneity to be constant over space, for example if housing policy and mortgage loans only vary over time but not over space. If the heterogeneity occurs over both space and time, we should consider both individual and time fixed effect, called a two-ways fixed model. For housing studies, the individual-fixed model is typically an ideal specification, as the heterogeneity varies across space for each observation. However, if the housing policy or mortgage rate is important in the study objective and changes dynamically over time, a two-ways fixed effect model should be specified. Generally, in a short-run horizon, both government and finance factors are constant, and the 
estimated coefficients of the fixed-effects models should not be biased because of omitted time-variant characteristics. For example, floor area, numbers of room, distance to local amenity, etc. in an individual-fixed model can control for all time-invariant differences between the individuals.

If the sample size is large, a fixed effects model would be domain to deal with the issue of enormous loss of degrees of freedom. Compared with fixed effected model, the random effects model also can avoid this issue, which assumes that the variation is random and uncorrelated with the independent variables. The random effect model is:

$$
y_{i t}=\alpha_{0}+\beta_{i t}^{T} x_{i t}+u_{i}+\varepsilon_{i t}
$$

Where:

$\mathrm{u}_{\mathrm{i}}$ is independent of the $\varepsilon_{\text {it }}$.

$\mathrm{x}_{\mathrm{it}}$ is independent of the $\mathrm{u}_{\mathrm{i}}$ and ${ }^{\varepsilon}{ }_{\text {it }}$.

Note that the random effects model might be appropriate if observations are representative of a sample rather than the whole population. In that case, there will be a "group" effect that can be observed in that group to be samples from a larger population. For example, if surveying students are in different campuses then"campus" may be a random effect, which allows for time-invariant variables to play a role as explanatory variables.

The choice between fixed and random effect model? In fact, the fixed versus random effects issue has generated a hot debate in the statistics literature. "...the crucial distinction between fixed and random effects is whether the unobserved individual effect embodies elements that are correlated with the regressors in the model, not whether these effects are stochastic or not" (Greene, 2008, p.183). If an entity's error term $u_{i}$ is correlated with the variables in $x_{i t}$, then the fixed effects model is 
appropriate. If an entity's error term $u_{i}$ is not correlated with the predictors $x_{i t}$, then we should choose random effect model as the ideal specification.

Generally, to decide between fixed or random effects, it is possible to run a Hausman-Wu test where the null hypothesis is that difference in coefficients are not systematic (Greene 2008). It basically tests whether the unique errors $u_{i}$ are correlated with the regressorsx $x_{i t}$, and the null hypothesis is that they are not correlated.

$$
H=\left(\hat{\beta}_{F E}-\hat{\beta}_{R E}\right)^{\prime}\left(\operatorname{Var}\left(\hat{\beta}_{F E}\right)-\operatorname{Var}\left(\hat{\beta}_{R E}\right)\right)^{-1}\left(\left(\hat{\beta}_{F E}-\hat{\beta}_{R E}\right)\right) \text { Equation (6.5) }
$$

Where

$\widehat{\beta}$ denotes the corresponding fixed effects estimates.

\subsection{Data and study area}

\subsubsection{Study area}

A review of the housing welfare policy in China shows that the development of real estate between 1949 and 1978 was very slow, due to little investment in housing construction. According to Chen and Gao (1993), less than 10\% of total capital investment was channeled into new housing construction during the period of the mid-1950s and late 1970s. However, after 1978, the government carried out various experiments of urban housing reforms, the purpose of government being to shift new housing provision from employers to the market (Wang and Murie 2000). The latest housing policy reform was launched in 1998 when the government abandoned the traditional housing allocation system. Since 1998, commodity residential housing became the main housing supply mode and dwellings were built by development companies and sold at full market price in the primary market. According to statistics, 
figures from the National Bureau of Statistic of China, show that investment in real estate development reached RMB1080.6 billion Yuan in2005, which was an increase of 22.9 per cent compared to the previous year, and show the average growth rate of investment into the Chinese real estate development to be 27.7 per cent from 1998 to 2005. Since 2005, the Chinese government adjusted housing policies five times in order to control house price increases rapidly and to restrict multiple property investment behavior

Nanjing (also known Nanking), China is chosen for this study, as it is a typical Chinese city, with a history of over 2000 years, it also being the ancient capital of China. Nanjing is therefore a good city in which to study the influence of infrastructure configurational changes and house prices: the stock of roads has changed dramatically at the same time as house prices.

Nanjing was the cultural and historical center of ancient China and is located in the east coast, two hours from the city of Shanghai by high-speed rail. Generally, It is considered as the second-largest city in the East China region, where Shanghai is the largest commercial center. Nanjing has been seventh place of ranking of "Cities with Strongest Comprehensive Strength" issued by the National Statistics Bureau, meanwhile, it is also considered as the second potential city for sustainable development in the Yangtze River Delta. The study area in Nanjing includes all ten districts within the metropolitan region, namely Pukou, Gulou, Jiangning, Xuanwu, Jianye, Xixia, Qinhuai, Yuhua, Baixia and Xiaguan, with a total population of over 5.3 million and a land area of $6597 \mathrm{~km}^{2}$. From statistical year book records, Nanjing has experiencedfast growth, with the GDP and population of the municipality growing from 14.2 billion RMB and 4.88 million in 1988 to 381.462 billion RMB and 6.24 million in 2008, respectively. Like other capital cities of the32 provinces in China, every year there are hundreds and thousands off armersmigrating into the city, looking for jobs. As shown in table (6.1), from 2005 to 2007, the population of the 
metropolitan area increased by 1 million people each year, but after 2008 , only three million people moved each year, indicating that the urbanization process has slowed down. Furthermore, large amounts of green land have been transformed into urban lands for residential purposes. For example, from 2005 to 2007,building area increased by 800 ha annually, and after the finance crisis of 2007, the government extended the building area to 1030 ha in an attempt to stabilize the employment rate. With respect to transportation infrastructure construction, street network development has not simply grown by radial sprawl in the urban fringe; there have also been urban regeneration projects in inner city areas due to land use transformation. Table (6.1), shows that there have been nearly $200-300 \mathrm{~km}$ of road infrastructure projects completed each year, including the freeway, $1^{\text {st }}$ grade road, 2 rd grade road, $3^{\text {rd }}$ grade road and $4^{\text {th }}$ grade roads. In year 2006alone, $800 \mathrm{~km}$ length was completed. The classification of road infrastructure shows that fastest growing type of road is $4^{\text {th }}$ grade roads which increased from $4812 \mathrm{~km}$ to $6216 \mathrm{~km}$ over the period. Second grade roads follow this and in contrast with freeways and third grade roads, which increased at a slower speed. 


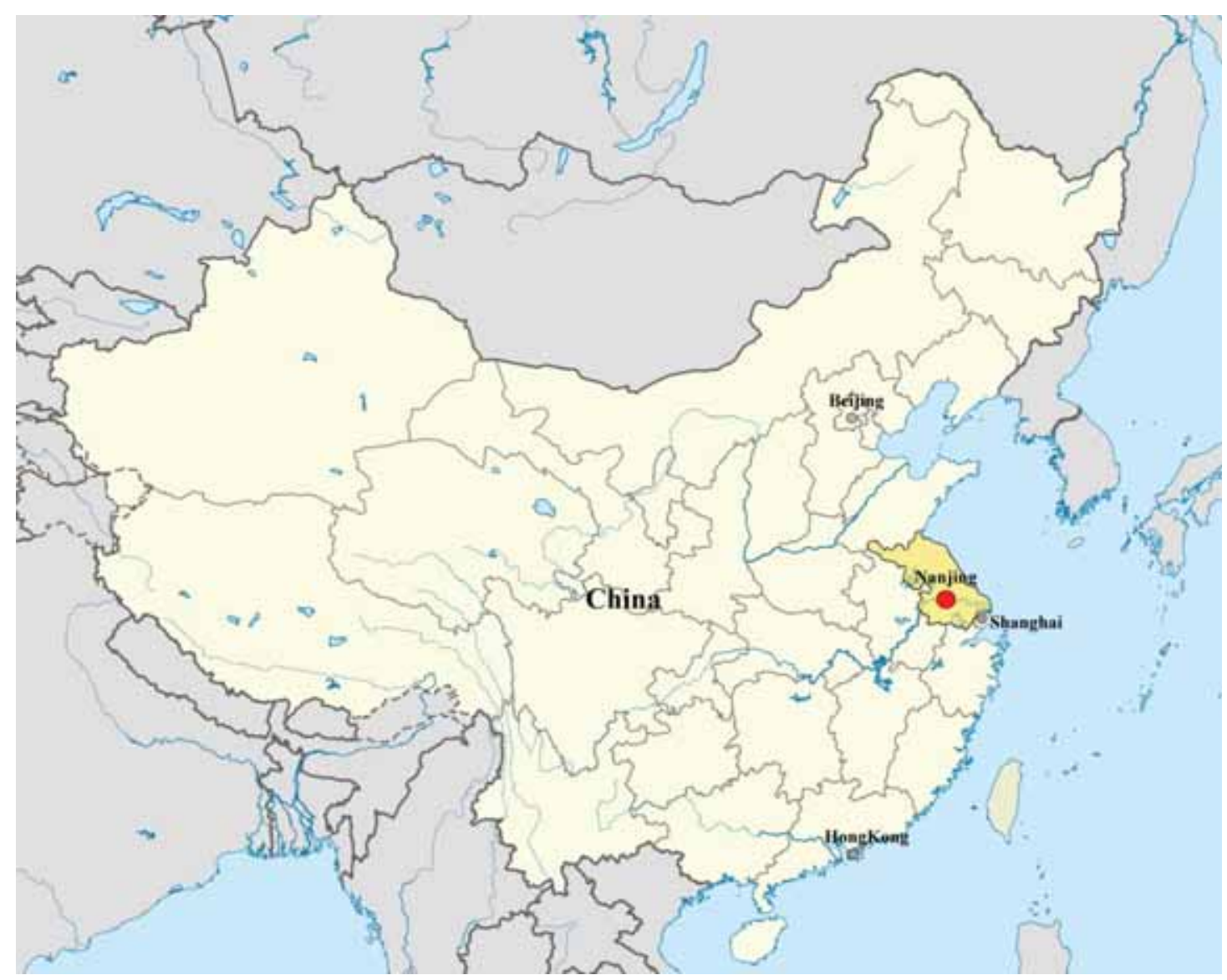

Figure 6.1 Location of Nanjing in China

Source: Author 


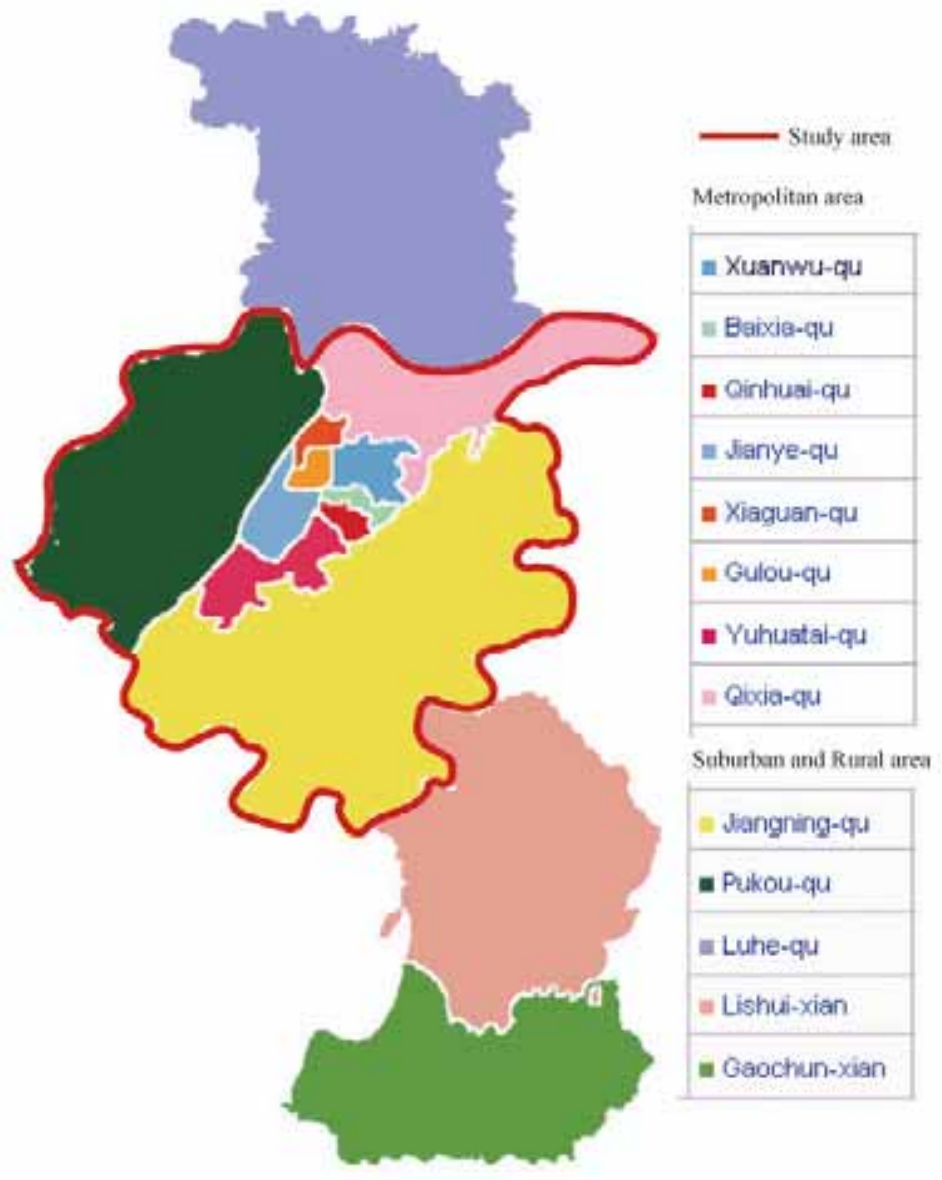

Figure 6.2 Study area of Nanjing

\section{Source: Author}

Table 6.1 General information of Nanjing from 2005-2010

\begin{tabular}{|c|c|c|c|c|c|c|c|}
\hline & & 2005 & 2006 & 2007 & 2008 & 2009 & 2010 \\
\hline \multicolumn{2}{|c|}{ Population of Metropolitan areas (million) } & 513.80 & 524.64 & 534.39 & 541.24 & 545.97 & 548.37 \\
\hline \multirow{2}{*}{\multicolumn{2}{|c|}{ Land areas of Metropolitan areas }} & 4723.0 & 4723.07 & 4723.07 & 4723.07 & 4723.07 & 4723.07 \\
\hline & & 7 & & & & & \\
\hline \multicolumn{2}{|l|}{ Housing price index } & 107.8 & 104.3 & 106.6 & 102.7 & 98.9 & 103.8 \\
\hline \multicolumn{2}{|c|}{ New increased building area of housing (Hectares) } & 842.15 & 816.85 & 746.69 & 1030.75 & 1350.50 & 819.57 \\
\hline infrastructure & Total & 8805 & 9688.9 & 9947 & 10164 & 10509 & 10749 \\
\hline \multirow[t]{5}{*}{ construction (km) } & Freeway & 309 & 390.5 & 400 & 430 & 434 & 480 \\
\hline & $1^{\text {st }}$ grade & 268 & 386.6 & 523 & 534 & 771 & 775 \\
\hline & $2^{\text {nd }}$ grade & 909 & 1073.6 & 1148 & 1236 & 1289 & 1404 \\
\hline & $3^{\text {rd }}$ grade & 445 & 578 & 754 & 768 & 766 & 762 \\
\hline & $4^{\text {th }}$ grade & 4812 & 5657.6 & 5677 & 5866 & 6044 & 6216 \\
\hline
\end{tabular}




\subsubsection{Data sources}

Panel data at micro level are very rare even in industrial countries, because it has to be collected repeatedly for the same individual over time. This is why the panel data employed in this study is collected from a survey conducted by author. There are two main components of this survey: urban morphology (street network) maps and both property value and property's attributes from 2005 to 2010 .

\subsubsection{Street network maps}

The street network raw data of Nanjing Metropolitan areas from 2005 and 2010was collected from a Chinese GPS map company. As the digital map they used is for Sat Nav purposes, it has accurate street network information with proper geo-coordinates for each year, so that it can capture accurate dynamic changes of the urban street network within the metropolitan area. Figure (6.3) shows the street network changes within each administration area by overlapping a time series of six annual maps. From the composite map, it is seen how infrastructure construction re-shapes the urban street layout in a very short space of time; for example, Hexi and Jiangning new town developments in 2006, interior ring way construction in 2005, Nanjing Yangzi River Third Bridge in 2006 and Xuanwu lake tunnel in 2006. 


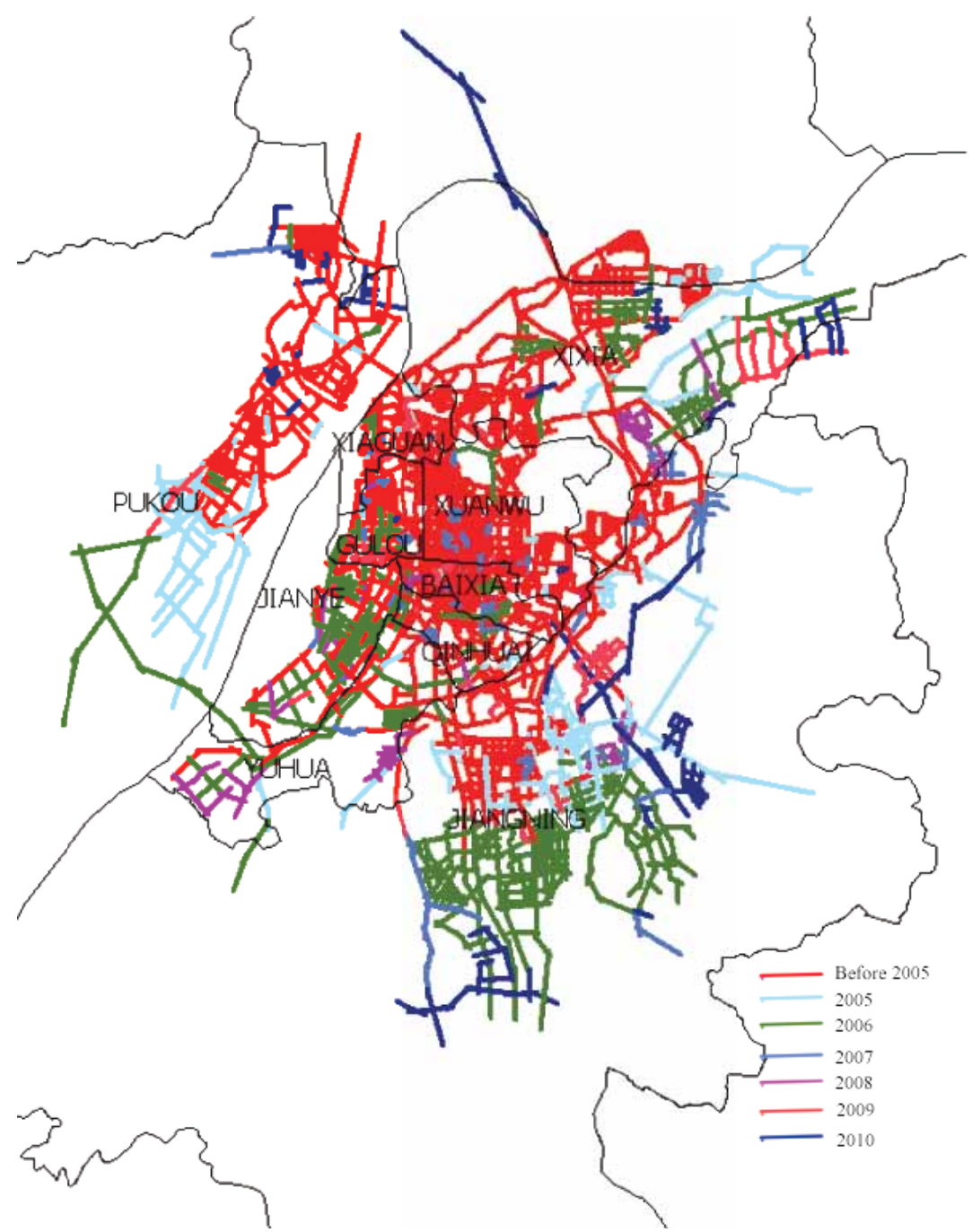

Figure 6.3 The changes of urban configuration in Nanjing from 2005-2010

Source: Author

\subsubsection{Street network analysis}

Another data source is a space syntax analysis of these street network maps. Based on prior experience, I specify 10 ranges of radii from $800 \mathrm{~m}$ to infinite distance as space syntax connectivity zones. In order to capture the dynamics of accessibility contained in the evolving urban street layout, space syntax analysis was administered separately for the street network maps from 2005 to 2010. Also note that the accessibility value attached to individual residential house price observation is the value of street segment nearest to the observation. Furthermore, spatial analysis is also employed to 
calculate straight-line distance to the CBD (XinJie Kou), distance to the XuanWu lake and distance to the ZhongShan park. 


\subsubsection{House prices and attributes}

The analyzed data includes average selling price by spatial unit (residential project) from 2005 to 2010 in Nanjing and was collected by the author from two Chinese professional real estate website databases (www.sofun.com and www.fangjia.com). The dataset includes for each project: information on the average selling price of developments (ASP), the date of valuation, administration area, project units size, gross-area, parcel-size, dummy variable of new blank of decoration, plot ratio, green ratio, numbers of parking units and service maintenance fee. Although the survey collected the ASP of individual residential development projects repeatedly from 2005 to 2010,there is still a missing data issue, due to missing attribute value and ASP for some projects. The dataset is unbalanced in terms of sample size by each year, which is not equal. There are 229 observations of projects in 2005 and observations of projects increase to 592 in 2010, showed in table (6.3). This is because some projects are newly developed and they do not have historical ASP. There are 2704 valid observations in the study. The sample was not randomly selected, but the sample size is more than $90 \%$ of the population within the metropolitan area. Furthermore, it was commodity residential housing developments with simplex residential land use (flats) that were also selected for observation, with developments with mixed land use and affordable housing excluded from the study. The ASP recorded is the instant ASP in October of each year. According to experience, October is the peak time for transaction for the whole year, so the ASP in that month is a good indicator of supply and demand.

Table 6.3 The changes of mean of housing price from 2005 to 2010

\begin{tabular}{llllll}
\hline \hline 2005 & 2006 & 2007 & 2008 & 2009 & 2010 \\
$\mathrm{~N}=229$ & $\mathrm{~N}=308$ & $\mathrm{~N}=447$ & $\mathrm{~N}=540$ & $\mathrm{~N}=588$ & $\mathrm{~N}=592$ \\
\hline 5047.65 & 6096.64 & 7684.15 & 9292.93 & 11131.74 & 15073.59 \\
\hline
\end{tabular}


The dependent variables require more discussion. Most hedonic price studies use individual house transaction price as the dependent variable to measure people's wiliness to pay extra money for the specific characteristics in the hedonic model. However, this study applies average selling price, estimated by the developer, instead of using the housing transaction price that is used in most hedonic price studies. There are several pragmatic reasons for using the average selling price. Firstly, in mainland China, transaction price information for new build sales is mainly controlled by government, and is not released to the public. The registration systems for sales of new houses and second-hand houses are in separate departments, and it is very difficult to gain public access to the records of individual dwelling units. Secondly, the Chinese housing market is not mature, as private property has been commercialized only since1998, and there are few cases of properties transacted more than two times. This means that even if historical transaction price data were available, it would not be possible to construct a panel data set of transaction prices for the purposes of our study. I have therefore chosen to take average selling price as a surrogate and accept the noise that this inevitably introduces into the model.

Average selling price $\left(y u a n / \mathrm{m}^{2}\right)$ is the average price of a residential project for the first transaction between developer and house purchasers. Because of the way the residential development market works in China, this metric is a commonly understood indicator of market performance. In the Chinese housing market, ASP is calculated by real estate developers weekly or monthly, to measure housing demand and supply, along with constructions cost and profits, and is an important reference for house purchaser in understanding the dynamic of the market. Although the average selling price is an advertised price, according to developer's expectation, it is also used to guide government taxation. According to research by Tian (2006), the formula of average selling price is: 


$$
\text { Average selling price }=\frac{\sum f_{i} P_{i}}{\sum f_{i}}
$$

Where,

$f_{i}$ is the floor space of the $\mathrm{I}^{\text {th }}$ apartment.

$P_{i}$ is selling price per square meter of floor space of the $\mathrm{I}^{\text {th }}$ apartment.

Table (6.4) shows the statistics descriptive of both dependent variable and independent variables, with total 2704 transaction records,

Table 6.4 Statistics descriptive data

\begin{tabular}{|c|c|c|c|c|c|c|c|}
\hline \multirow{2}{*}{$\begin{array}{l}\text { Variable } \\
\text { PRICE }\end{array}$} & \multirow[b]{2}{*}{ overall } & \multirow{2}{*}{$\begin{array}{l}\text { Mean } \\
9.063\end{array}$} & \multirow{2}{*}{$\begin{array}{l}\text { Std. } \\
\text { Dev. } \\
0.541\end{array}$} & \multirow{2}{*}{$\begin{array}{l}\text { Min } \\
7.305\end{array}$} & \multirow{2}{*}{$\begin{array}{l}\text { Max } \\
\qquad 11.472\end{array}$} & \multicolumn{2}{|c|}{ Observations } \\
\hline & & & & & & $\mathrm{N}=$ & 2704 \\
\hline & between & & 0.433 & 7.922 & 10.461 & $\mathrm{n}=$ & 593 \\
\hline & within & & 0.340 & 7.975 & 11.149 & T-ba & $=4.55987$ \\
\hline \multirow[t]{3}{*}{ YEAR } & overall & 2008.008 & 1.574 & 2005.000 & 2010.000 & $\mathrm{~N}=$ & 2704 \\
\hline & between & & 0.683 & 2007.500 & 2010.000 & $\mathrm{n}=$ & 593 \\
\hline & within & & 1.455 & 2005.008 & 2010.508 & T-bal & $=4.55987$ \\
\hline \multirow[t]{3}{*}{ BAIXIA } & overall & 0.096 & 0.295 & 0.000 & 1.000 & $\mathrm{~N}=$ & 2704 \\
\hline & between & & 0.304 & 0.000 & 1.000 & $\mathrm{n}=$ & 593 \\
\hline & within & & 0.000 & 0.096 & 0.096 & T-bat & $=4.55987$ \\
\hline \multirow[t]{3}{*}{ GULOU } & overall & 0.132 & 0.339 & 0.000 & 1.000 & $\mathrm{~N}=$ & 2704 \\
\hline & between & & 0.338 & 0.000 & 1.000 & $\mathrm{n}=$ & 593 \\
\hline & within & & 0.000 & 0.132 & 0.132 & T-bat & $=4.55987$ \\
\hline \multirow[t]{3}{*}{ JIANYE } & overall & 0.136 & 0.343 & 0.000 & 1.000 & $\mathrm{~N}=$ & 2704 \\
\hline & between & & 0.340 & 0.000 & 1.000 & $\mathrm{n}=$ & 593 \\
\hline & within & & 0.000 & 0.136 & 0.136 & T-bat & $=4.55987$ \\
\hline \multirow[t]{3}{*}{ JIANGNING } & overall & 0.202 & 0.401 & 0.000 & 1.000 & $\mathrm{~N}=$ & 2704 \\
\hline & between & & 0.397 & 0.000 & 1.000 & $\mathrm{n}=$ & 593 \\
\hline & within & & 0.000 & 0.202 & 0.202 & T-ba & $=4.55987$ \\
\hline \multirow[t]{3}{*}{ PUKOU } & overall & 0.103 & 0.304 & 0.000 & 1.000 & $\mathrm{~N}=$ & 2704 \\
\hline & between & & 0.297 & 0.000 & 1.000 & $\mathrm{n}=$ & 593 \\
\hline & within & & 0.000 & 0.103 & 0.103 & T-bat & $=4.55987$ \\
\hline \multirow[t]{3}{*}{ QINHUAI } & overall & 0.071 & 0.256 & 0.000 & 1.000 & $\mathrm{~N}=$ & 2704 \\
\hline & between & & 0.245 & 0.000 & 1.000 & $\mathrm{n}=$ & 593 \\
\hline & within & & 0.000 & 0.071 & 0.071 & T-bal & $=4.55987$ \\
\hline \multirow[t]{3}{*}{ XIXIA } & overall & 0.075 & 0.264 & 0.000 & 1.000 & $\mathrm{~N}=$ & 2704 \\
\hline & between & & 0.270 & 0.000 & 1.000 & $\mathrm{n}=$ & 593 \\
\hline & within & & 0.000 & 0.075 & 0.075 & T-bal & $=4.55987$ \\
\hline \multirow[t]{2}{*}{ XIAGUAN } & overall & 0.058 & 0.233 & 0.000 & 1.000 & $\mathrm{~N}=$ & 2704 \\
\hline & & & 189 & & & & \\
\hline
\end{tabular}




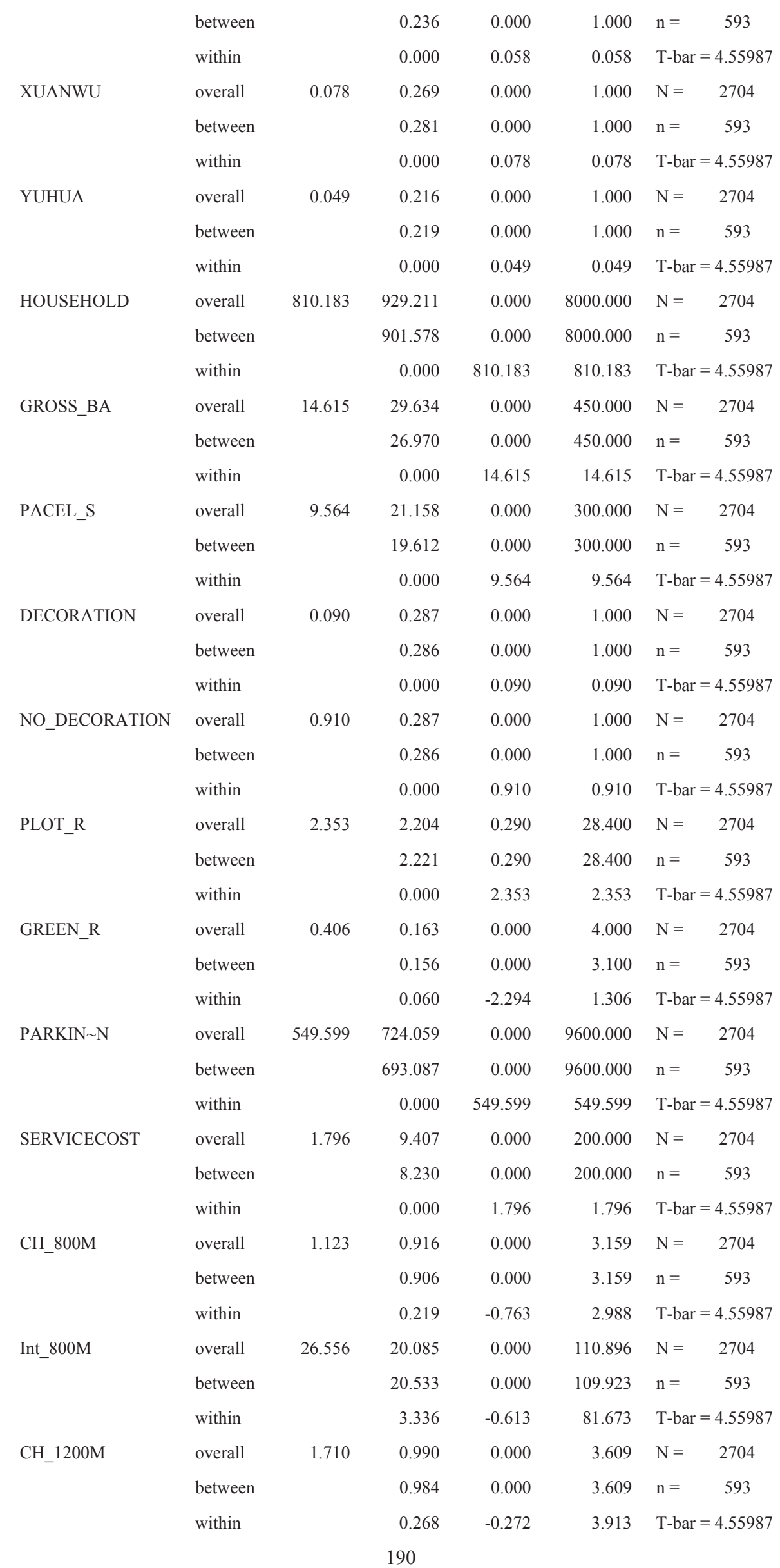




\begin{tabular}{|c|c|c|c|c|c|c|c|}
\hline \multirow[t]{3}{*}{ Int_1200M } & overall & 45.818 & 38.459 & 0.000 & 225.211 & $\mathrm{~N}=$ & 2704 \\
\hline & between & & 39.664 & 0.000 & 219.794 & $\mathrm{n}=$ & 593 \\
\hline & within & & 5.627 & -5.382 & 97.018 & T-bar & 4.55987 \\
\hline \multirow[t]{3}{*}{$\mathrm{CH} \_2 \mathrm{KM}$} & overall & 2.378 & 1.102 & 0.000 & 4.353 & $\mathrm{~N}=$ & 2704 \\
\hline & between & & 1.107 & 0.000 & 4.348 & $\mathrm{n}=$ & 593 \\
\hline & within & & 0.291 & 0.108 & 5.062 & T-bar & 4.55987 \\
\hline \multirow[t]{3}{*}{ Int_2KM } & overall & 94.781 & 82.232 & 0.000 & 458.834 & $\mathrm{~N}=$ & 2704 \\
\hline & between & & 84.512 & 0.000 & 457.025 & $\mathrm{n}=$ & 593 \\
\hline & within & & 9.488 & 16.944 & 152.862 & T-bar & 4.55987 \\
\hline \multirow[t]{3}{*}{$\mathrm{CH} \_5 \mathrm{KM}$} & overall & 3.372 & 1.383 & 0.000 & 5.549 & $\mathrm{~N}=$ & 2704 \\
\hline & between & & 1.388 & 0.000 & 5.546 & $\mathrm{n}=$ & 593 \\
\hline & within & & 0.388 & 0.096 & 7.062 & T-bar & 4.55987 \\
\hline \multirow[t]{3}{*}{ Int_5KM } & overall & 347.858 & 248.767 & 4.861 & 1200.282 & $\mathrm{~N}=$ & 2704 \\
\hline & between & & 254.354 & 4.861 & 1192.670 & $\mathrm{n}=$ & 593 \\
\hline & within & & 27.734 & 55.974 & 481.674 & T-bar & 4.55987 \\
\hline \multirow[t]{3}{*}{$\mathrm{CH} \_8 \mathrm{KM}$} & overall & 3.748 & 1.520 & 0.000 & 6.101 & $\mathrm{~N}=$ & 2704 \\
\hline & between & & 1.518 & 0.000 & 6.095 & $\mathrm{n}=$ & 593 \\
\hline & within & & 0.449 & 0.011 & 7.928 & T-bar & 4.55987 \\
\hline \multirow[t]{3}{*}{ Int_8KM } & overall & 598.556 & 344.517 & 5.491 & 1503.338 & $\mathrm{~N}=$ & 2704 \\
\hline & between & & 349.923 & 5.491 & 1494.876 & $\mathrm{n}=$ & 593 \\
\hline & within & & 44.854 & 164.825 & 804.564 & T-bar & 4.55987 \\
\hline \multirow[t]{3}{*}{ CH_10KM } & overall & 3.894 & 1.578 & 0.000 & 6.278 & $\mathrm{~N}=$ & 2704 \\
\hline & between & & 1.570 & 0.000 & 6.272 & $\mathrm{n}=$ & 593 \\
\hline & within & & 0.477 & -0.029 & 8.220 & T-bar & 4.55987 \\
\hline \multirow[t]{3}{*}{ Int_10KM } & overall & 731.524 & 371.984 & 5.491 & 1595.801 & $\mathrm{~N}=$ & 2704 \\
\hline & between & & 376.150 & 5.491 & 1584.911 & $\mathrm{n}=$ & 593 \\
\hline & within & & 54.704 & 180.075 & 924.251 & T-bar & 4.55987 \\
\hline \multirow[t]{3}{*}{ CH_12KM } & overall & 4.005 & 1.615 & 0.000 & 6.378 & $\mathrm{~N}=$ & 2704 \\
\hline & between & & 1.605 & 0.000 & 6.369 & $\mathrm{n}=$ & 593 \\
\hline & within & & 0.499 & -0.279 & 8.443 & T-bar & 4.55987 \\
\hline \multirow[t]{3}{*}{ Int_12KM } & overall & 841.348 & 383.721 & 6.995 & 1683.363 & $\mathrm{~N}=$ & 2704 \\
\hline & between & & 386.375 & 6.995 & 1666.133 & $\mathrm{n}=$ & 593 \\
\hline & within & & 63.160 & 209.702 & 1044.529 & T-bar & 4.55987 \\
\hline \multirow[t]{3}{*}{$\mathrm{CH} \_15 \mathrm{KM}$} & overall & 4.121 & 1.664 & 0.000 & 6.513 & $\mathrm{~N}=$ & 2704 \\
\hline & between & & 1.650 & 0.000 & 6.504 & $\mathrm{n}=$ & 593 \\
\hline & within & & 0.526 & -0.332 & 8.613 & T-bar & 4.55987 \\
\hline \multirow[t]{3}{*}{ Int_15KM } & overall & 972.804 & 372.772 & 8.961 & 1809.427 & $\mathrm{~N}=$ & 2704 \\
\hline & between & & 373.027 & 8.961 & 1789.510 & $\mathrm{n}=$ & 593 \\
\hline & within & & 71.336 & 279.579 & 1220.005 & T-bar & 4.55987 \\
\hline \multirow[t]{3}{*}{ CH_20KM } & overall & 4.238 & 1.719 & 0.000 & 6.779 & $\mathrm{~N}=$ & 2704 \\
\hline & between & & 1.699 & 0.000 & 6.769 & $\mathrm{n}=$ & 593 \\
\hline & within & & 0.559 & -0.650 & 8.736 & T-bar & 4.55987 \\
\hline \multirow[t]{2}{*}{ Int_20KM } & overall & 1126.919 & 331.764 & 102.371 & 1914.174 & $\mathrm{~N}=$ & 2704 \\
\hline & between & & 327.436 & 132.767 & 1885.313 & $\mathrm{n}=$ & 593 \\
\hline
\end{tabular}




\begin{tabular}{|c|c|c|c|c|c|c|}
\hline & within & & 82.454 & 408.373 & 1454.174 & T-bar $=4.55987$ \\
\hline \multirow[t]{3}{*}{ CH_N } & overall & 4.289 & 1.818 & 0.000 & 6.965 & 2704 \\
\hline & between & & 1.785 & 0.000 & 6.954 & 593 \\
\hline & within & & 0.594 & -0.986 & 9.588 & $\mathrm{~T}-\mathrm{bar}=4.55987$ \\
\hline \multirow[t]{3}{*}{ Int_N } & overall & 1260.245 & 249.667 & 554.104 & 1902.368 & 2704 \\
\hline & between & & 233.892 & 587.327 & 1861.653 & 593 \\
\hline & within & & 91.787 & 569.353 & 1532.228 & T-bar $=4.55987$ \\
\hline \multirow[t]{3}{*}{ DIST_CBD } & overall & 8.626 & 0.754 & 5.441 & 10.620 & 2704 \\
\hline & between & & 0.782 & 5.441 & 10.620 & 593 \\
\hline & within & & 0.000 & 8.626 & 8.626 & T-bar $=4.55987$ \\
\hline \multirow[t]{3}{*}{ DIST_LAKE } & overall & 8.844 & 0.579 & 6.900 & 10.659 & 2704 \\
\hline & between & & 0.594 & 6.900 & 10.659 & 593 \\
\hline & within & & 0.000 & 8.844 & 8.844 & T-bar $=4.55987$ \\
\hline \multirow[t]{3}{*}{ DIST_PARK } & overall & 9.089 & 0.432 & 7.681 & 10.561 & 2704 \\
\hline & between & & 0.439 & 7.681 & 10.561 & 593 \\
\hline & within & & 0.000 & 9.089 & 9.089 & T-bar $=4.55987$ \\
\hline
\end{tabular}

\subsection{Analysis and empirical results}

\subsubsection{Street network analysis}




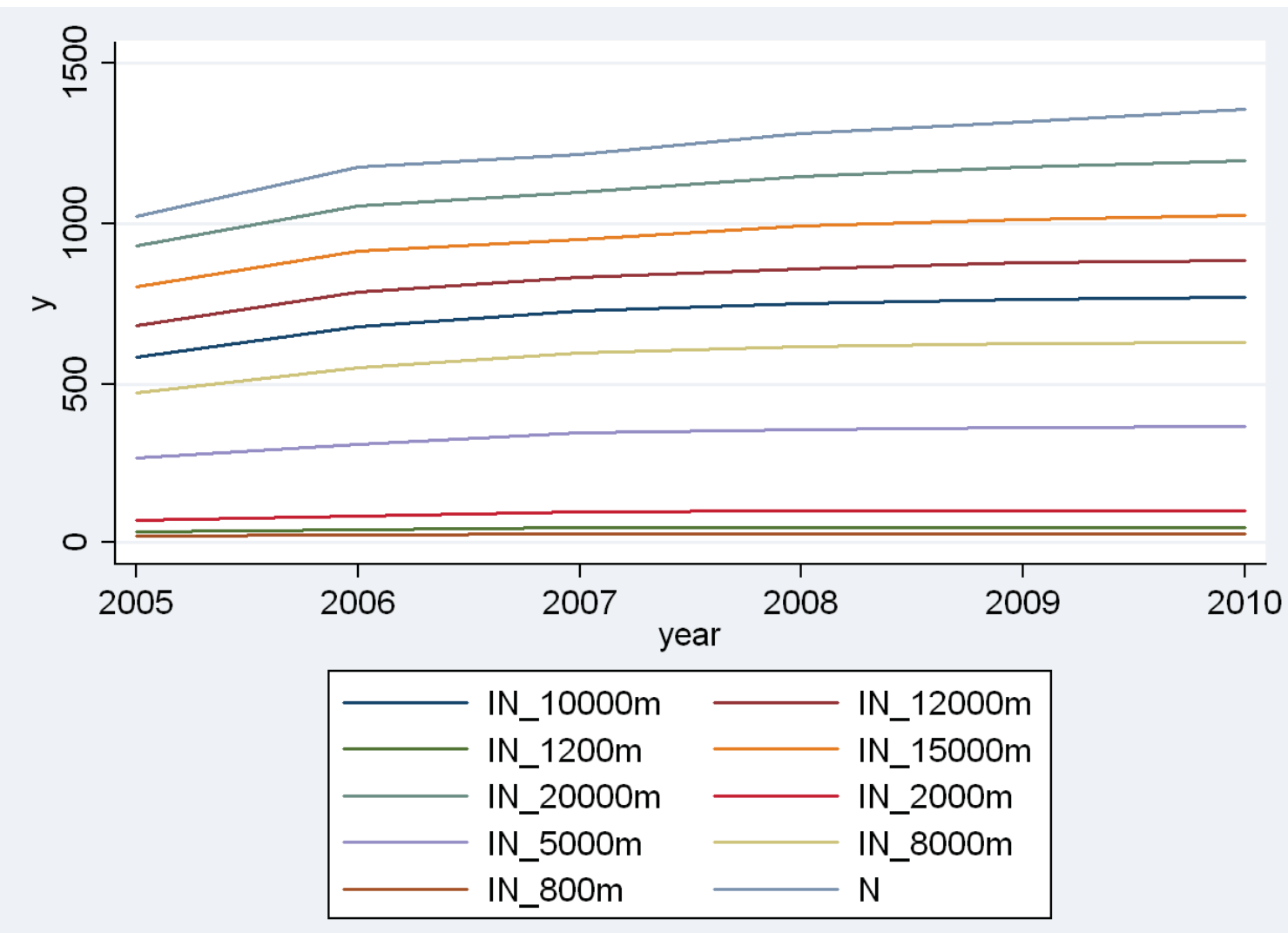

Figure 6.4 Integration value change from 2005-2010 at different radii

Source: author 


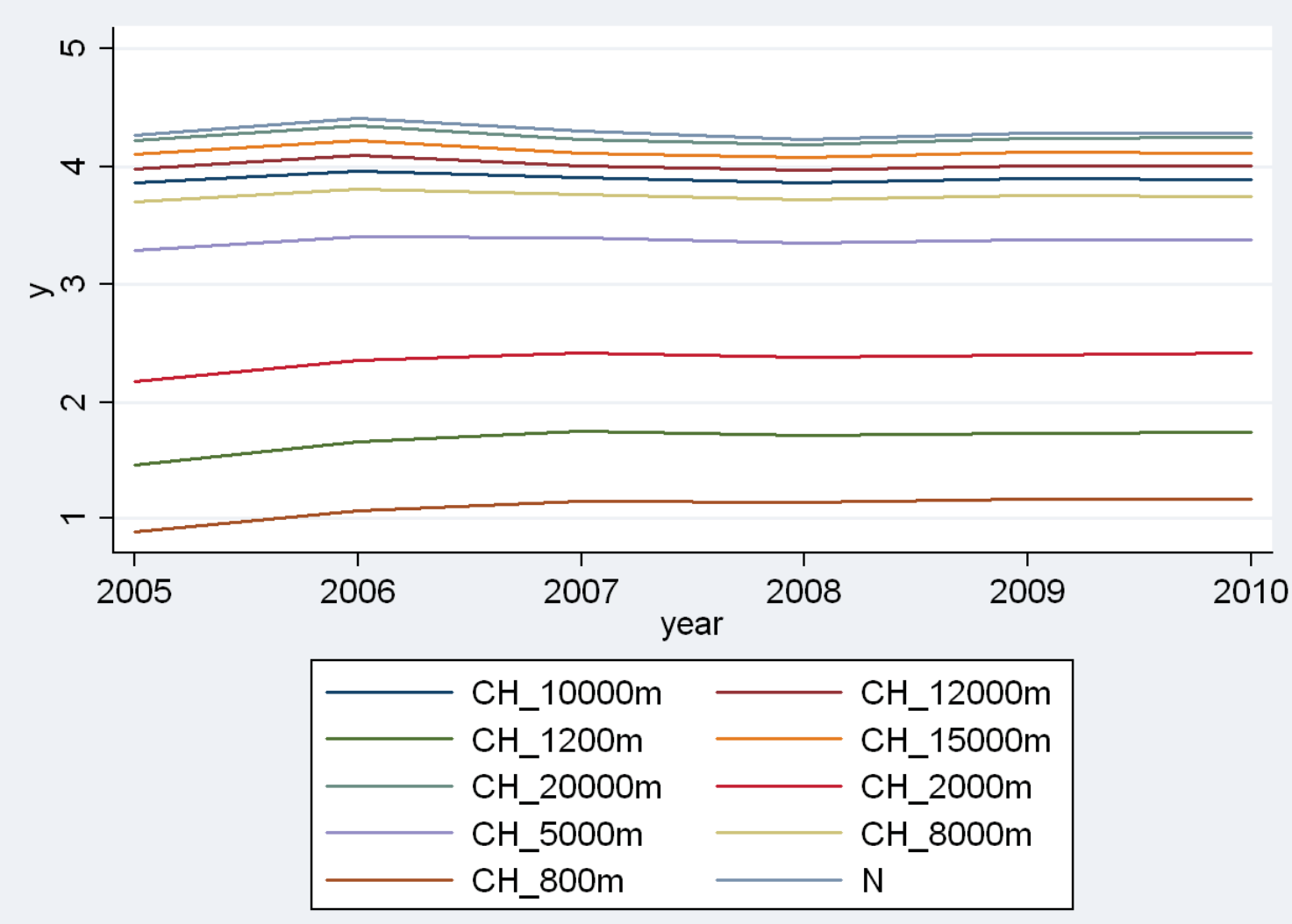

Figure 6.5 Choice value change from 2005-2010 at different radii

Source: author

There are 120 variables of urban morphology features applied in this chapter, which are all visualized in Appendix-6 respectively. Generally, compared to the case of Cardiff, the integration value of Nanjing in all the years show a single agglomeration hub, which is exactly located in the city center, and the effect area become larger with the search radii increasing. By contrast, it is expected to see that the choice value redistributes dynamically with infrastructure improvements, such as new bridge and tunnel. With regards to the changes of urban configuration features in temporal, Figure (6.4) shows the mean change for integration value at different radii. It is seen that when less than $5 \mathrm{~km}$ radii, the integration value are stable over years, which may be because less infrastructure projects occurred with the radii of $5 \mathrm{~km}$ to the city center. However, when the above the radii $8 \mathrm{~km}$, the means of integration value grow year by year, in particular from 2005 to 2006, which indicates that various of street 
network improvements enhanced the city connectivity, especially the urban suburb area. Relatively, choice value shows two types of changes trend, in particular during the period of 2005 to 2007 (Figures 6.5). Within the radii of $2 \mathrm{~km}$, the mean of the choice value increase, whereas, all average choice value decrease slightly when above the radii of $5 \mathrm{~km}$, which implies that the series of main road improvements have brought relief to the congestion. In that case, the traffic flows increased in the areas surrounding the center and is reduced in the area of suburb area.

\subsubsection{Empirical results}

Bearing in mind that the objective of this study is to examine the micro-dynamic effect of street network configuration on housing price using a panel model. There are five forms of models employed in this study: pooled model, individual-fixed effect model, random effect model, time-fixed model and two-ways effect fixed model, all using average selling price as the dependent variable and 40 explanatory variables, including 20 urban configuration attributes and 20 time-invariance attributes (e.g. distance to $\mathrm{CBD}$, project units size, gross-area, parcel-size, decoration condition, plot ratio, green ratio, parking units size and service maintenance fee). As mentioned in the methodology section, the fixed effects model can control for time invariance by absorbing time invariant variables in the intercept. The random effect model deals with time invariant components as explanatory variables. Thus, the time-invariance attributes are excluded in individual-fixed effect model, time-fixed model and two-way effect model. The exploratory process of panel analysis will be decomposed into seven components, which are as follows:

Table 6.5 Empirical results of five models

\begin{tabular}{|c|c|c|c|c|c|c|c|c|c|c|}
\hline \multirow[t]{2}{*}{ Variables } & \multicolumn{2}{|c|}{ OLS } & \multicolumn{2}{|c|}{ Fixed } & \multicolumn{2}{|c|}{ Random } & \multicolumn{2}{|c|}{ Time-fixed } & \multicolumn{2}{|c|}{ Two-ways } \\
\hline & Ceof. & $t$ value & Ceof. & $t$ value & Ceof. & $t$ value & Ceof. & $t$ value & Ceof. & $t$ value \\
\hline Intercept & 10.055 & $23.559 * *$ & & & 10.868 & $16.594 * *$ & & & & \\
\hline CH_800M & -0.009 & -0.385 & 0.083 & $2.138^{*}$ & 0.032 & 1.083 & 0.001 & 0.054 & -0.002 & -0.063 \\
\hline
\end{tabular}




\begin{tabular}{|c|c|c|c|c|c|c|c|c|c|c|}
\hline Int_800M & 0.002 & 1.114 & 0.002 & 0.966 & 0.002 & 1.09 & 0.001 & 0.563 & 0.000 & 0.14 \\
\hline $\mathrm{CH}_{-} 1200 \mathrm{M}$ & -0.016 & -0.425 & -0.011 & -0.232 & 0.012 & 0.291 & -0.007 & -0.232 & 0.034 & 1.051 \\
\hline Int_1200M & -0.001 & -1.096 & -0.003 & $-2.221 *$ & -0.002 & $-1.771^{\#}$ & -0.001 & -1.432 & 0.000 & -0.382 \\
\hline $\mathrm{CH} \_2 \mathrm{KM}$ & 0.155 & $3.811 * *$ & -0.041 & -0.725 & 0.093 & $1.968 *$ & 0.041 & 1.24 & -0.038 & -0.985 \\
\hline Int_2KM & -0.001 & $-1.674^{\#}$ & 0.000 & 0.018 & -0.001 & -1.728 & 0.000 & 0.722 & 0.000 & 0.352 \\
\hline CH_5KM & 0.048 & 0.729 & 0.191 & $2.292 *$ & 0.152 & $2.070 *$ & 0.058 & 1.107 & 0.057 & 0.991 \\
\hline Int_5KM & -0.001 & $-2.150 *$ & 0.001 & 1.461 & 0.000 & -0.303 & 0.000 & -1.952 & -0.001 & $-1.858^{\#}$ \\
\hline CH_8KM & -0.217 & -1.527 & -0.416 & $-2.293 *$ & -0.390 & $-2.420 *$ & -0.270 & $-2.33 *$ & -0.068 & -0.541 \\
\hline Int_8KM & 0.000 & 0.746 & -0.001 & 0.746 & -0.001 & -1.127 & 0.000 & -1.127 & 0.001 & 1.061 \\
\hline CH_10KM & 0.103 & 0.531 & 0.023 & 0.102 & 0.123 & 0.588 & 0.002 & 0.014 & -0.072 & -0.454 \\
\hline Int_10KM & 0.000 & 0.683 & -0.002 & $-2.073 *$ & 0.000 & -0.428 & 0.003 & $6.016^{* *}$ & 0.000 & 0.355 \\
\hline $\mathrm{CH}_{-} 12 \mathrm{KM}$ & 0.144 & 0.71 & 0.290 & 1.429 & 0.217 & 1.104 & 0.077 & 0.462 & 0.032 & 0.231 \\
\hline Int_12KM & -0.002 & $-4.235 * *$ & -0.001 & $-1.797^{\#}$ & -0.001 & $-1.842^{\#}$ & -0.002 & $-4.903 * *$ & 0.000 & 0.265 \\
\hline CH_15KM & -0.215 & -1.4 & 0.001 & 0.004 & -0.215 & -1.499 & 0.152 & 1.209 & 0.051 & 0.509 \\
\hline Int_15KM & 0.001 & $3.896 * *$ & 0.000 & 0.779 & 0.000 & 0.721 & 0.001 & $2.477^{*}$ & 0.000 & 0.348 \\
\hline $\mathrm{CH}_{-} 20 \mathrm{KM}$ & 0.059 & 0.618 & 0.014 & 0.169 & 0.120 & 1.406 & -0.105 & -1.343 & 0.006 & 0.096 \\
\hline Int_20KM & -0.001 & $-5.567 * *$ & -0.001 & $-4.199 * *$ & -0.001 & $-4.129 * *$ & 0.000 & 0.199 & 0.000 & 0.091 \\
\hline CH_N & -0.039 & -1.229 & -0.130 & $-3.474 * *$ & -0.103 & $-3.004 * *$ & 0.018 & 0.693 & 0.007 & 0.256 \\
\hline Int_N & 0.003 & $20.825 * *$ & 0.005 & $31.668 * *$ & 0.004 & $31.521 * *$ & 0.000 & $-4.493 * *$ & 0.000 & $-1.956^{\#}$ \\
\hline BAIXIA & 0.304 & $5.606 * *$ & & & 0.285 & $3.194 * *$ & & & & \\
\hline GULOU & 0.467 & $9.444 * *$ & & & 0.578 & $7.050 * *$ & & & & \\
\hline JIANYE & 0.274 & $6.251 * *$ & & & 0.360 & $4.899 * *$ & & & & \\
\hline JIANGNING & -0.113 & $-2.351 *$ & & & -0.105 & -1.36 & & & & \\
\hline PUKOU & -0.105 & $-1.905^{\#}$ & & & 0.111 & 1.259 & & & & \\
\hline QINHUAI & 0.237 & $4.819 * *$ & & & 0.349 & $4.178 * *$ & & & & \\
\hline XIXIA & 0.091 & 1.465 & & & -0.055 & -0.55 & & & & \\
\hline XIAGUAN & 0.197 & $3.646 * *$ & & & 0.250 & $2.778 * *$ & & & & \\
\hline XUANWU & 0.286 & $4.699 * *$ & & & 0.226 & $2.284 *$ & & & & \\
\hline Units size & 0.000 & $-4.514 * *$ & & & 0.000 & $-4.009 * *$ & & & & \\
\hline GROSS_BA & -0.001 & $-2.065 *$ & & & 0.000 & -0.012 & & & & \\
\hline PACEL_S & 0.003 & $3.351 * *$ & & & 0.002 & 1.441 & & & & \\
\hline New Blank & 0.142 & $5.239 * *$ & & & 0.162 & $3.506 * *$ & & & & \\
\hline PLOT_R & -0.003 & -0.729 & & & -0.010 & -1.467 & & & & \\
\hline GREEN_R & 0.182 & $3.804 * *$ & & & 0.081 & 1.362 & & & & \\
\hline P_UNIT & 0.000 & $4.029 * *$ & & & 0.000 & $2.895 * *$ & & & & \\
\hline MAIN_FEE & 0.002 & $2.299 *$ & & & 0.003 & $1.742^{\#}$ & & & & \\
\hline D_CBD & -0.310 & $-9.223 * *$ & & & -0.418 & $-8.203 * *$ & & & & \\
\hline D_LAKE & 0.035 & 1.114 & & & 0.027 & 0.519 & & & & \\
\hline D_PARK & -0.049 & -1.137 & & & -0.149 & $-2.119 *$ & & & & \\
\hline R-Squared & \multicolumn{2}{|r|}{0.509} & \multicolumn{2}{|c|}{0.658} & \multicolumn{2}{|c|}{0.844} & \multicolumn{2}{|c|}{0.418} & \multicolumn{2}{|c|}{0.01} \\
\hline Adj. R-Squared & \multicolumn{2}{|r|}{0.501} & \multicolumn{2}{|c|}{0.509} & \multicolumn{2}{|c|}{0.831} & \multicolumn{2}{|c|}{0.414} & \multicolumn{2}{|c|}{0.008} \\
\hline F-statistic & \multicolumn{2}{|r|}{69.051} & \multicolumn{2}{|c|}{200.972} & \multicolumn{2}{|c|}{359.388} & \multicolumn{2}{|c|}{96.35} & \multicolumn{2}{|c|}{1.074} \\
\hline p-value & \multicolumn{2}{|r|}{0} & \multicolumn{2}{|r|}{0} & \multicolumn{2}{|r|}{0} & \multicolumn{2}{|c|}{0} & \multicolumn{2}{|c|}{0.37} \\
\hline
\end{tabular}

Signif.codes: $\quad 0$ ‘**’ $0.01^{\text {‘ } * ’ 0} 0.05^{\text {‘\# }} 0.1$ 
Firstly, ordinary least squares (OLS) was performed for pooled regression models, assuming the coefficients for any individual at any cross-section do not vary in time. The tests for the OLS model in figure (6.5), shows that Adj.-R-Squared is 0.501, F test is 69.051 , and the $\mathrm{p}$ value is 0.000 , which indicates that the model is statistically significant. There is a linear dynamic relationship between street configuration accessibility and housing price with $40.6 \%$ variance explained by this model. With the exception of three areas, namely XIXIA,PUKOU and JIANGNING, the T scores of all others areas are above 1.96 with a positive sign, which indicates the relationship between accessibility and housing price in these areas is stable over the years. Furthermore, as expected, distance to CBD ,project units size and gross-building area are significant, all with negative signs, whereas parcel size, decoration status, green space ratio, numbers of parking spaces, and service cost are significant with positive signs. These findings suggest that projects nearer to the CBD, with fewer households, bigger parcel size, good decoration, more green space, more parking space and high service cost (indicating better/more club goods) are associated with higher price.

In terms of urban configurational attributes, the space syntax choice (betweenness) coefficient only has a significant (positive) impact at radii $2 \mathrm{~km}$. This implies that the locations of greatest people movement measured within $2 \mathrm{~km}$ travel distance is associated with higher house prices. This may be picking up local agglomeration effects at a neighborhood level, with highest movement potential from a $2 \mathrm{~km}$ catchment generating sufficient neighborhood economies of scale to attract home-value enhancing facilities, possibly including metro stations.

By contrast, the integration(closeness) value, measuring access to opportunities, shows a significantly negative impact at radii $5 \mathrm{~km}, 12 \mathrm{~km}$ and $20 \mathrm{~km}$, but positive impact at $15 \mathrm{~km}$ and $\mathrm{N} \mathrm{km}$. This apparently arbitrary pattern seems to indicate that high value property is found in a mix of less and more accessible places. There is a 
non-monotonic relationship between closeness and house price but there are significant relationships between house price and specific levels of closeness. It is difficult to think of specific explanations for these patterns, but it must be a function of the particular configuration of Nanjing and its recent extensions. One explanation might be that 5,12 and $20 \mathrm{~km}$ radii do not correspond to significant search areas for any particular kind of destinations demanded by higher income residents - on the contrary, they may correspond to search areas for low-paid jobs, which would account for the negative coefficients. It might also be noted that the global integration value has the largest $\mathrm{T}$ score (20.825), reflecting the fact that most high value projects are located at places that are easy to access from across the city. In Nanjing as elsewhere, people pay for accessibility to transaction opportunities at different scales.

Next, I performed a fixed effects model (also known as individual-fixed), which allows that unobserved heterogeneity between individual project prices to vary across space, while parameters $\beta$ are constant. Table (6.5), shows that the model is statistically significant $(\mathrm{p}<0.05)$ and adj.-R-Squared is 0.509 , confirming that a dynamic relationship between street network improvement and housing price exists. In the fixed effects model, the space syntax choice (betweenness) values, indicating the relative level of traffic flow in street segment, are significant at radii $800 \mathrm{~m}$ and $5 \mathrm{~km}$ with positive signs, and also significant at radii $8 \mathrm{~km}$ and $\mathrm{N} \mathrm{km}$ with negative signs. This is again suggesting interesting subtle patterns in the spatial economy: within $5 \mathrm{~km}$ area, the high value properties are located near main streets where the pedestrian flows are high. $800 \mathrm{~m}$ betweenness hotspots are attractive places to live by virtue of access to a high density of local walking-based destinations. Betweenness hotspots at $8 \mathrm{~km}$ and $\mathrm{N} \mathrm{km}$ have high traffic externalities that are not compensated for by local services.

On the other hand, as the p-values of integration (closeness) values measured at radii $1200 \mathrm{~m}, 10 \mathrm{~km}$ and $20 \mathrm{kmarelower}$ than 0.05 , and the all of their T-tests are negative, 
there is evidence that some at least of the high value properties are not located at easily accessed place, such as near highways and elevated roads. This may well reflect the phenomenon of high valued exclusive gated developments on the urban periphery. Nevertheless, the more significant pattern is for high value properties to be located at places of easy access, with the t value of global integration being largest of all.

Based on the results of the individual-fixed effect model, it can be inferred that street network improvements at a small scale (e.g. improving street density and development of small new-town developments), tend to add value to residential projects, whereas larger scale street network improvement (e.g. freeways, express ways and bridges) tend to impose negative externalities(noise, fumes and insecurity) on housing, which are capitalized in asking price. Furthermore, the results also confirm the benefit to housing price from both inner and outer ring roads, as these decentralize the street connectivity from the original city center, adding value to properties further out.

Table 6.6 F test for individual effects

\begin{tabular}{lll}
\hline \hline \multicolumn{3}{c}{ F test for individual effects } \\
\hline $\mathrm{F}=9.5741 \quad \mathrm{df1}=573 \quad \mathrm{df} 2=2090$ & p-value $<2.2 \mathrm{e}-16$ \\
alternative hypothesis: significant effects & \\
\hline
\end{tabular}

The third step is to test whether the panel model controls the unobserved determinants efficiently. If the fixed model is better than the OLS model, we should use the panel structure, otherwise the pooled model should be used. A simple Chow test is used to check whether the fixed model is significantly different to the OLS model, showed in table (6.6). If the p-value is $<0.05$ then the fixed effects model is a better choice. In this case, as the p-value is much lower than 0.05 , we can reject the null hypothesis and choose the fixed model. 
The fourth step is to run a random effects model. The crucial distinction between fixed and random effects is whether the unobserved individual effects embody elements that are correlated with the regressors in the model (Greene 2008). As table (6.5) shows, the Random effects model is also significant with a much higher adj.-R-Squared: $83.1 \%$ of variation being explained by this model.

Looking at the time-invariant variables, the dummy variables for five admin areas are found to be significant at 95\% confidence: BAIXIA, GULOU, JIANYE, QINHUAI and XIAGUAN, which is to be expected: Figure (6.3) clearly shows the larger numbers of street network improvements in these administration areas, which have attracted private estate developers to develop luxury residential projects for high-income people as the location became more accessible. This is particularly strong in the GULOU area. Furthermore, the variable 'unit size' is significant at $99 \%$ level with a negative sign, whereas decoration condition is significant with a positive sign and number of parking spaces is also significant with a positive impact on house prices. This implies that projects in the high-end market have the characteristics of lower density, better decoration and more parking space near the city center, whereas the projects for the general market are associated with large numbers of units, new blank decoration and less parking space.

In addition, it was found that the attributes of total building area, parcel size, plot-ratio and green ratio are not significant statistically, indicating interestingly that high value of ASP are not related to development density. As expected, both the distance to CBD and distance to a major park are significant with a negative sign, but the variable of distance to lake dropped out of the model. This may be due to the fact that although the lake is free for public, it is gated and there are only six entrances. Thus the XUANHU lake appears to have no spillover effect on house prices.

In terms of urban morphology attributes, betweenness (choice) value at radii $2 \mathrm{~km}$ and 
$5 \mathrm{~km}$ are significant with a positive sign, and the choice values at radii $8 \mathrm{~km}$ and globe are significant with negative sign. As I have already suggested when discussing the other models, this shows distinctive spatial boundaries for different kinds of demand. Within $5 \mathrm{~km}$ area, high pedestrian flows have a positive externality on housing price, since they imply higher vitality and more welfare-enhancing destinations catering for foot-born custom. Higher betweenness over $5 \mathrm{~km}$ is associated with higher automobile traffic and associated negative externalities.

Integration values are statistically significant only at radii $20 \mathrm{~km}$ and globally, with different signs, underlining the existing of submarkets. As has already been noted, many high value properties are located in places less accessible within radii $20 \mathrm{~km}$, but some types of such developments buck this trend in the urban fridge area.

Table 6.7 Hausman Test

\begin{tabular}{lc}
\hline \hline & Hausman Test \\
\hline chisq $=1029.051 \quad \mathrm{df}=20 \quad$ p-value $<2.2 \mathrm{e}-16$ \\
alternative hypothesis: one model is inconsistent \\
\hline
\end{tabular}

The next step is to choose between the fixed effect model and the random effects model. As noted in the methodology section, the fixed effects model only describes the relationship within selected samples, while the random effects model could be interpreted to larger populations. The Hausman-Wu test (Hausman, 1978) was employed to make the choice, based on whether the unobserved individual effects elements are correlated with the repressors in the model. If the p-value is significant (for example $<0.05$ ) then the fixed effects model should be used(Greene 2008).From table (6.7), the chi-sq is 1029.051 and p-value is lower than 0.05 , so the fixed effect model is better than the random model.

The sixth step is to examine the relationship by time-fixed-effects model, which 
allows unobserved heterogeneity (e.g. inflations and mortgage rate) to vary only across time, and ignores the heterogeneity of individuals across space. If the model is significant statistically, it indicates that the rate of price change for the whole market for each year is not equal.

The model turns out to be statistically significant, with an adj.-R-Squared of 0.414 and $\mathrm{F}$ test of 96.350 , showed in table (6.6), which indicate that the price change rate of the whole market for each year is different. Compared with the performance of urban configurational attributes in the individual-effect model, it is found that integration or choice values are hardly significant within the radii at $5 \mathrm{~km}$, which suggest that the housing price volatility within the old town has little relationship with the change of urban configuration statistically. This is as expected, as few large size street layout improvements occurred in the old town during the study periods, as seen in figure (6.3). However, the Choice value is significant at radii $8 \mathrm{~km}$ with a negative sign, indicating that within the specific area of people's travel distance range (between $5 \mathrm{~km}-8 \mathrm{~km})$, property value increased in some location where the choice value decreased during 2005 to 2010 . The reasons may be that the specific area is located in an area where the old town and the new town intersected, and normally the ring road and expressways are the means to link the old and new town. After these links had been constructed, people started to choose the ring road or express way for minimum travel time, so that the original choice value decreases, as traffic volume are mainly shifted to the ring road and express ways.

Integration values are significant at radii $12 \mathrm{~km}$ and globally with negative $\mathrm{t}$ values, while $10 \mathrm{~km}$ and $15 \mathrm{~km}$ integration values have positive signs. This implies that there are two opposite relationships between housing price change and integration change outside of the old town, and the ring road is the spatial boundary that divides these two relationships. Since the ring road is located far from the city center at a network integration distance of $10 \mathrm{~km}$ and $12 \mathrm{~km}$. It is known that building the ring road could 
enhance central growth and allow people living in the suburb areas or city center to quickly access both areas. Thus, house price increases are associated with integration increases in suburb areas, new town and within the old town. However, regarding the area within the inner ring road, the integration value is similar with choice value, and has decreased in value since the ring road was constructed. The ring road enables people to commute within the inner ring road, especially when two places are positioned diagonally. The time-fixed model shows how housing price volatility is also associated with spatial accessibility change across time and the results conform to the dynamic relationship between housing price and street accessibility. This also suggest that the relationship within the inner ring road is opposite to that beyond the inner ring road. However, the time-fixed effect model cannot reflect how the volatility of different markets (high-end or low-end) follows the changes of urban configuration..

Thus, a chow test and Lagrange Multiplier Test were used to examine further whether the data needs a two-way effect model, which allows the unobserved heterogeneities to vary across both space and time (e.g. people's expectation, housing stocks, and business circumstance of each developer). The two-way effect model can be used to examine whether the dynamic relationship over space is synchronous with that over time, which means that a bigger price movement caused by a bigger change of spatial accessibility can be found in the market of higher value property. The p-values of both Chow test and Lagrange Multiplier Test are smaller than 0.05, showed in table (6.8) and (6.9), which rejects the null hypothesis of no difference between individual-fixed effect and time-fixed effect model. Hence, a two-way fixed-effect model should be explored.

Table 6.8 F test for individual effects and time-fixed effect

\begin{tabular}{lll}
\hline \hline & F test for individual effects \\
\hline $\mathrm{F}=5.323$ & df1 $=587 \quad$ df2 $=2091 \quad$ p-value $<2.2 \mathrm{e}-16$
\end{tabular}


Table 6.9 Lagrange Multiplier Test

\begin{tabular}{l}
\hline \hline Time-fixed model \\
\hline Lagrange Multiplier Test - time effects (Honda) \\
normal $=433.4043 \quad$ p-value $<2.2 \mathrm{e}-16$ \\
alternative hypothesis: significant effects
\end{tabular}

However, the two-ways fixed effect model is insignificant, as it does not pass the F test with a p-value 0.370 , showed in table (6.5), indicating that the dynamic effect of accessibility on house price change across space and time is not synchronous. This was expected due to the result of both individual-fixed effect model and time-fixed effect model, as the signs of significant integration value in each model are opposite, which implies that the spatial pattern of housing price is not matched well with the pattern of change rate across time. Further, it indicates that there is no evidence proving that the higher value market also has a higher change rate due to the better change of spatial accessibility. Another reason for the two-ways fixed effect model not being significant could be due to the small sample size.

Based on these results, key findings will be summarized as follows.

Firstly, the existence of the dynamic effect of urban configuration on housing price volatility is confirmed by the individual-fixed effect model and time-fixed effect model, respectively. The individual-fixed model assumes that the change rate of each individual observation for each year is equal, while the time-fixed effect model assumes individual observations are homogenous across space. Generally, the results of the individual-fixed effect model show that high value property is located in areas with lower integration value and higher choice value within a range of radii $5 \mathrm{~km}$, and 
that this distribution is stable over 5 years; whereas, in time-fixed effect model, it is also found that the change of spatial accessibility can affect house price volatility. For example, in urban suburbs, increasing integration can increase property value, and within the ring road, decreasing choice value could also increase property value, (choice value at $8 \mathrm{~km}$ has a negative sign, and integration value at $10 \mathrm{~km}$ and $15 \mathrm{~km}$ has a positive sign). Although the two-way effect model is insignificant, it also indicates that housing price dynamics are asynchronous over space and time, and the rate of price change seems more related to location than to property value per se.

Secondly, the dynamic relationship between urban configuration and housing price is only valid for selected samples as the Hausman-Wu test rejected the null hypothesis, suggesting that the individual -fixed effect model is better fitted to this dataset than a random effect model.

Thirdly, the results imply the existence of housing submarkets as suggested, for example, by the finding that the dynamic relationship between urban configuration and housing price within the inner ring road is different from that beyond the inner ring road. This in turn suggests a positive dynamic relationship in the suburb area and a negative relationship in the buffer area.

\subsection{Discussion}

After the analysis, there are several points that need to be further discussed. Firstly, compared with traditional measurement for accessibility, the space syntax method is effective in capturing the accessibility changes at different levels of urban configuration at a disaggregated level. As the theory suggests, change of accessibility could be considered the most important geographical determinant of housing price movements (Capozza and Helsley 1989), however, few studies can empirically prove 
this hypothesis. One of the difficulties is to measure the change of accessibility accurately. For example, Iacono and Levinson (2011) conclude that the inappropriate measurement of accessibility could be a reason for models to be insignificant. However, this study confirms that the dynamic effect of urban configuration on housing price volatility exists. This allows, with appropriate modeling tools, for governments and investors to predict housing price pattern and movement by observing the levels and changes of accessibility across time and space. However, whether accessibility has a lagged effect on housing price still needs to be explored in greater detail.

In addition, it is found that not all types of road infrastructure improvements add value to the property, and people clearly have different preferences for road connectivity and traffic flow in central urban areas and in suburbs. For example, betweenness (choice) values tend to have positive signs at small radii, indicating people's willingness to pay extra money for intensely used streets within walking distance due, I suggest, to agglomeration economy effects of local amenities, rather than street segment connectivity per se. In contrast, a series of large scale street network improvements has been implemented by the government, rendering the city more accessible, but the evidence shows that it has a negative impact on people's private wealth and welfare, as both integration and choice show negative signs at large radii and thus also negatively impact on the housing price. Consequently it is possible to infer that people prefer high traffic flow in the central city and less connectivity and traffic flow in suburb areas.

Thirdly, the significant space syntax attributes at different radii could reflect the existence of housing neighborhood submarkets, and a dynamic relationship between these submarkets. The results of the time-fixed effect model suggests that housing price volatility is not affected by change of urban configuration in the city centre. There is a negative relationship between urban configuration and housing price, 
mainly found in the area within the ring road within a $2 \mathrm{~km}$ buffer distance. The change of urban configuration stimulated house prices to increase in the area beyond the ring road, This is because the improvement of the ring road enhanced the connections between city center and suburbs. However, this also reduced the connection and increased the traffic volume in a $2 \mathrm{~km}$ buffer area round ring road, as the two space syntax indices show a different sign at different radii. Furthermore, the two-way effect model is insignificant, which implies that the price change rate of high value property is not similarly high. Thus, the price change rate is related with location rather than the property value. It can be concluded that with the benefit of urban growth, house prices of submarkets in suburban areas are more likely to be impacted by accessibility changes.

Finally, it can be noted when comparing this chapter with the previous one, that people from different countries have different preferences, and different purchasing characteristics with respect to the urban configurations of housing properties. As the results of the Cardiff case showed in chapters 3and 4, high price property is associated with high street connectivity and low traffic flow, and high-income people do not care about how street segments connect and the state of traffic flow in suburb areas of the UK. However, the Chinese study shows that high price property is associated with high traffic flow in city center and both low connectivity and traffic flow in suburb area. This may be due to the different life styles or travel behaviors resulting indifferent preferences for urban configurations. Thus, it is suggested that further studies are needed to explore the relationship between urban configuration and housing price in different types of cities.

\subsection{Summary}

Despite the large numbers of studies exploring the relationship between location and land value, there is still remarkably little evidence on how this relationship evolves 
over time and space. Furthermore, the traditional hedonic statistical methods and hybrid repeat sale and hedonic methods fail to detect an accurate relationship over time, especially when the unobserved heterogeneity varies over time, due to, for instance housing policy and mortgage rate changes. The objective of this study was to explore the geographical determinants of housing price movement at a city scale, examining whether there is a dynamic effect of urban configuration on housing price change by using a longitudinal analysis, which has typically been employed in studies examining the determinants of house price volatility at a macro level. A location-based social network analysis method, known as space syntax, was applied to measure the accessibility contained in urban morphology. Then I conducted a survey, collecting street network map data, housing values, and related attributes for the metropolitan areas of Nanjing from 2005 to 2010.The statistical evidence suggests that the accessibility information contained in an urban configuration network graph is an important geographical determinant influencing housing price dynamics over space and time, respectively. Dynamics caused by urban street network infrastructure improvements have both positive and negative impacts on housing price overtime. Generally, accessibility measured using closeness as the indicator across the city(connectivity to everywhere else in the whole city) has a negative impact on prices. The betweenness value has a positive impact on the city center and a negative impact on the urban suburb. Furthermore, responding to changes of accessibility caused by the construction of the ring way, there is a negative dynamic relationship within the ring road and positive dynamic relationship beyond the ring road. But there is no evidence to suggest that places with high accessibility also have a high change rate, as the correlations is dependent on the particular case of urban morphology change in each housing submarket. 


\section{Chapter Seven:}

\section{Conclusions}

\subsection{Introduction}

The focus of this thesis is on urban morphology and property markets. In particular, it investigates the externalities of urban configuration on housing price, asking whether households are more willing to pay extra for a more generally accessible location when they purchase a property without a specific motivation and travel destination. The purpose of this final chapter is to summarise the key findings of previous chapters. It is divided into four sections. In addition, the chapter discusses policy implications of the thesis findings, including the importance of the research with respect to the hedonic price theory as well as the role of space syntax theory, and more generally spatial network analysis, and as a method to aid the precision of government and private sector planning and management of the built environment. I then comment on the study's limitation with respect to the issue of econometric estimation and the handling of space syntax radii. The final section provides suggestions for how the research can be further developed and will highlight directions for future research. Finally, some final conclusions for this entire thesis are offered.

\subsection{Conclusions for each chapter}

Chapter two presented a literature review of hedonic price modeling, which can be summarized from a number of aspects. Firstly, it is generally accepted in the literature that under the conditions of a perfectly competitive market, where demand equals supply, people's willing to pay for each characteristic of property becomes the 
implicitly measured attribute of the hedonic price model. Existing economic theories do not, however, provide clear guidelines on how to select hedonic price functional form. Moreover, hedonic modeling also suffers from a number of econometrics issues, which also lead to biased estimation, such as spatial heteroscedasticity, spatial autocorrelation and multicollinearity. The rest of chapter two mainly focused on reviewing relevant literature about locational attributes that have an impact on housing price. It was noted from the literature that urban growth as well as polycentric renders the urban system progressively more complex and that most people choose a location without considering minimum travel cost to employment centre. Consequently, the role of the distance to CBD in theory and in hedonic studies has less impact than earlier theories suggest. Finally, I suggest that the methods of measuring accessibility is overly simple as they mainly rely on the Euclidean distance where as disaggregated measurements requires a prior specification of destinations. It was also noted that by using Euclidean distance, even of polycentric centres, hedonic modeling studies ignore the potentially rich source of information contained in a city's road grid pattern.

Chapter three presented the space syntax methodology and its underlying algorithms. There are some inconsistencies in its early topological distance measurement, but a series of improvement such as metric segment analysis and geometric angular cost segment analysis, have improved the accuracy with which it measures general spatial accessibility. The literature also implies that metric segment analysis is suitable for large-scale analysis.

There are two mainly in dices used in space syntax metric segment analysis, both measuring the accessibility contained in urban configuration. The first attribute is integration (closeness) and the second is choice (betweenness). Integration can be interpreted as how well connected or how close one street segment is to all other street segment within the search radius. This measurement can indicate a proxy for the 
cost of overcoming spatial separation within a network of road segments. Choice can indicate the pattern of movements through the network within a specific radius. However, space syntax theory has not delineated the restrictive criterion on how to specify a search radii, which depends on the researcher's purpose and scale of study objective. Thus, the specification of search radii for each empirical study is pre-defined individually, usually in an exploratory fashion (ie without a priori hypotheses) but also usually taking as a rule of thumb, the association of certain radial distances with certain transport modes. In most cases radii are chosen for their assumed association with people's travel behavior by walking, cycling and vehicular transport. Although there exist a large number of studiessuggesting that housing price pattern is highly correlated with urban morphology, few studies test the hypothesis that urban street network morphology is a fundamental geographical determinant of house price (and therefore of the configuration of agglomeration externalities).

Chapter four therefore tests whether urban configuration can be a geographical determinant of house prices at both disaggregated and aggregated level. It uses the UK city of Cardiff as a case study. Compared to conventional geometrical measures of accessibility, the results imply that a network approach to measuring accessibility in urban areas improves the model's performance with respect to explanatory power and a reduction in heteroscedasticity, spatial autocorrelation and multi-collinearity. Through estimating space syntax accessibility variables across a variety of spatial scales, the chapter demonstrates that the study area displays a bi-centric urban configuration with respect to property prices that corresponds to local and city-wide externalities. The results show thaturban network configuration has a statistically significant impact on house price at both aggregated and disaggregated levels. Integration and choice both significantly affect house price for disaggregated data; integration value has a positive impact on house price whereas choice value has a negative impact. In regards to aggregated data, integration remains significantly positively correlated with house price, whilst choice value becomes insignificant. Due 
to the particular pattern of the street network in the case study city of Cardiff, with many dead-end streets linked directly to main streets, there is a higher variance for choice. However, this is lost at the aggregate level. Finally, the experiment shows that detailed urban configuration indicators measured from a network model of the urban grid had a stronger power in explaining variances of the house prices than the traditional measure of distance to $\mathrm{CBD}$ and without the scale limitation of that measure.

Chapter five provides new knowledge addressing the lack of understanding on how to deal with submarkets when the dwelling type is homogenous and neighbourhood characteristics are unstable over time. This chapter showed that urban configurational data recovered from a graph model of a street network adds significant explanation power in distinguishing between housing sub-markets and thus provides a potentially useful approach to analyzing sub-markets. This chapter used the same data set and study area presented in Chapter four. The whole process of identifying housing submarket followed the Schnare and Struyk procedure (1976), and compared the efficiency of two new alternative specifications. The first specification is optimal urban configuration and the second is nested urban configuration and building type with two traditional spatial specifications. The results show that all specifications using network metrics have significantly improved the estimation over and above the market-wide model. Although the specifications based on urban configurational features are not the best performing models, these results are quite close to the traditional specifications. In particular, the new alternative specification seems more objective, and less reliant on a housing market culture that has evolved around distinctly different building types. This suggests that it may be particularly useful in urban housing markets with a high degree of homogeneity in building type. Furthermore, the results also imply that different socio-economic classes have different preferences of urban morphology features. For example, people of the middle class cohort or above in Cardiff, tend to live further away from the city centre 
since they have access to cars and consequently are less sensitive to how well street segments are connected. This is in stark contrast to lower income people who seem more sensitive to the geometrical pattern of street segments.

Chapter six applies a network approach to hedonic modeling in a housing market dominated by simplex building types (Nanjing, China) and addresses the hypothesis that there is a micro-dynamic relationship between urban morphology and house price. It uses a large panel data set (of 2704 house prices) for the city of Nanjing, from 2005 to 2010. The statistical evidence suggests that the accessibility contained in urban configuration is an important geographical determinant influencing the housing price dynamic over space and time, respectively. Furthermore, urban street network infrastructure improvements have both positive and negative impacts on housing price over time. For example, integration has a negative impact on prices, while choice value has a positive impact in the city center and a negative impact in the urban suburb. This offers support for the idea that network metrics allow us to identify housing submarkets defined at least partially in relation to particular morphological characteristics - as predicted from the previous chapter. In particular, in the area surrounding the ring road in Nanjing, there is a negative dynamic relationship inside the ring road and a positive dynamic relationship beyond the ring road. However, there is no evidence that places with high accessibility also have a high change rate. The results imply that house prices in the suburbs are more likely to be effected by the dynamics of urban configuration. By comparing the results in chapter four and five, it is found that people from different countries have different preferences in regards to the urban configurations of residential properties. For example, in the UK context, high priced properties are associated with high street-connectivity and low traffic flow, and high-income people do not apparently care about the connectivity of street segments and the state of traffic flow in suburban areas of the UK. However, the Chinese study shows that high price properties are associated with high traffic flow in the city center and both low connectivity and low traffic flow in suburban areas. 


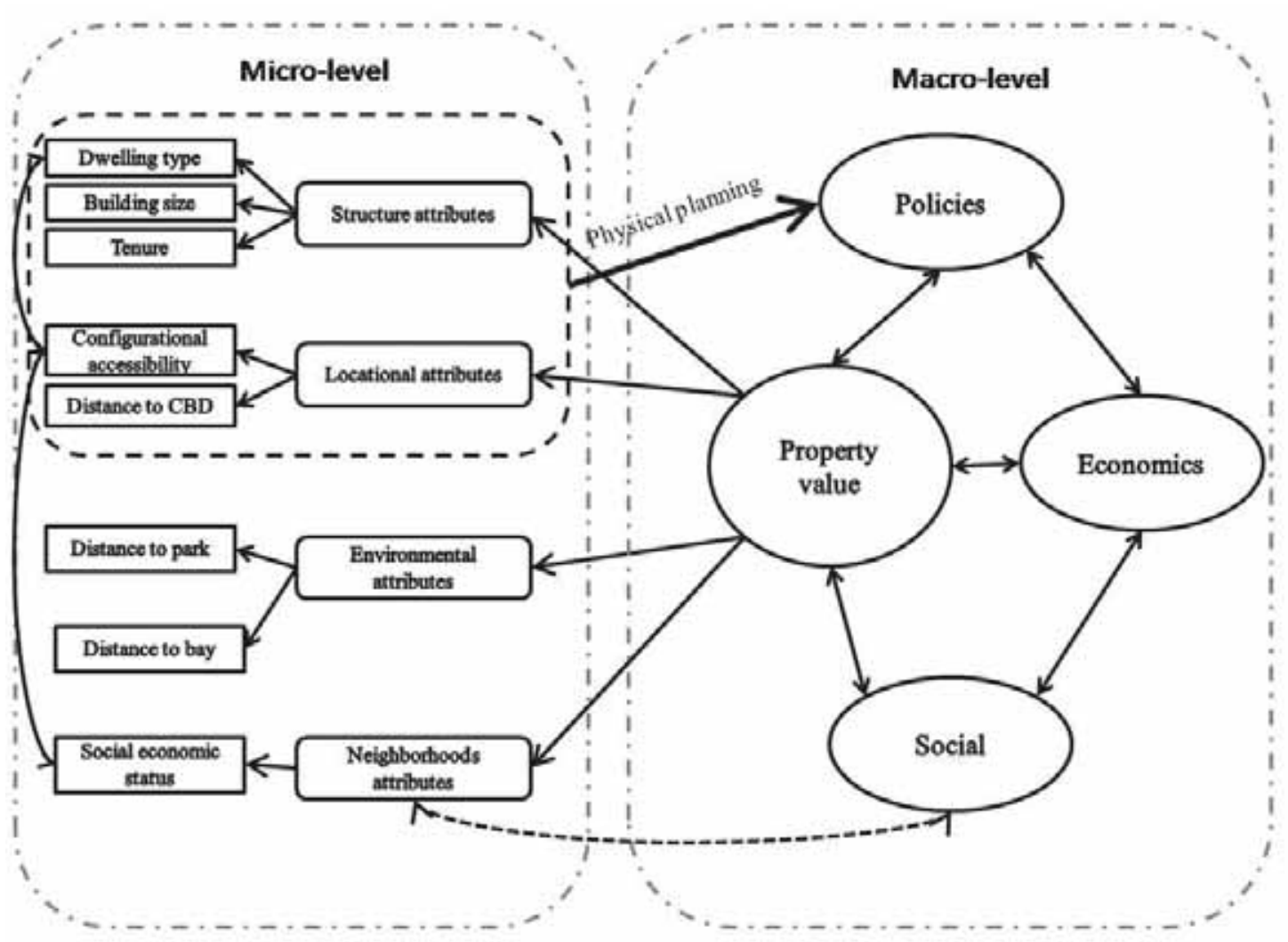

Figure 7.1 Diagrammatic structural equation of housing price

Source: author

In summary, this thesis attempts to contribute to the hedonic theory through its emphasis on housing price mainly at the micro level (Figure 7.1), and provides a framework for the study of urban morphology and housing market. Several empirical evidences show that the urban configurational features can have both positive and negative impacts on property value. The spatial information contained in the urban form has stronger explanations for the variance of housing price compared to the information of traditional locational attributes, and be more objective. Surprisingly, this study also found that urban configurational features are associated with structure characteristics and neighborhoods characteristics, and that each social class has specific preferences in terms of building type and street layout. Moreover, at the macro-level, it is known that the real estate is closely associated with social, economic and policy factors, but from the urban planning perspective, the studies on urban configuration may be a means to tackle some complex issues (e.g. social exclusion and housing segregations) through efficient allocations of space, following this framework here. 


\subsection{Implications}

This section discusses the implications of these findings from three perspectives: firstly, implications for the space syntax and related spatial network theory; secondly, implications for hedonic house price research; and thirdly implications for urban development and planning policy.

\subsubsection{Implications for the Space Syntax theory}

The findings of this dissertation have several implications for spatial network theories of cities such as Space Syntax, Place Syntax, sDNA and others. The main contribution of the thesis is to provide new evidence showing under what circumstances when choosing a property, spatial network accessibility is likely to influence people's locational preferences. In particular, it measures house buyers preference for being either close to or further from movement and opportunity. This supports the argument of Hillier and Hanson (1984) that people have an innate ability to read or comprehend the meanings of different arrangements or layout of space. Or from Webster's economic interpretation (2010), that street networks spatially distribute the general benefits that come from many people co-locating: they allocate agglomeration economies across space. However, the results of this thesis show in detail that the relationship between urban morphology and socio-economic activities tend to vary over space. The thesis examines this through investigating housing submarkets. Traditional space syntax studies do not touch upon this issue. For example, in chapters four and five, it is found that high income people only consider the house size rather than how a street segment is connected in suburb areas whereas low-income people are more sensitive to the street layout in the city center. This finding implies that estimation results of previous space syntax studies, such as in urban crime and social exclusion studies, are likely to be over estimated. It also supports Webster's (2010) insistence that Space Syntax only deals with general accessibility and is silent on 
specific accessibility (equivalent to weighted spatial network analysis).

Finally, the thesis has found that the specification of spatial network analysis radii should be based on the particulars of particular cases rather than on prior theoretical or methodological knowledge. Choice of analytical radii should be determined by a city's specific spatial features.

As for the topological distance measurement in space syntax theory, the Local integration (R3) measures accessibility up to three steps away. In terms of the axial line, the topological distance measurement suggests that integration at a local level correlates strongly with local pedestrian movement, and integration (R3) is applied to predict the local pedestrian movement for many cases(Hillier and Hanson 1984; Hillier 1996). However, this thesis has found that people from different countries have different preferences for urban configurational attributes of houses. Thus, it seems more appropriate to apply a specific space syntax index to each different case.

\subsubsection{Implications for Hedonic price theory}

The hedonic pricing method is very well established, particularly in North America, but few studies have focused on the UK and China. The theory suggests that distance to $\mathrm{CBD}$ has a negative effect on housing price and that its explanatory power is becoming weaker. One reason is that people's consumer motivation leading in the past towards minimum travel cost to work place, have changed as people are willing to pay extra for proximity to local amenities. This seems well established in US context. The implication of my work for hedonic price theory is that people's consumer motivation has also evolved in UK and China context, and that the negative gradient of CBD on property value is less significant than expected, particularly for high-income people. 
Furthermore, against the background of a weakening CBD-effect in hedonic modeling, the thesis has shown that street network indices of accessibility are very effective, as it does not require any specifications of people's motivation at the disaggregated level. Since people have different motivations and destinations, traditional measurements for accessibility at the disaggregated level requires a prior specification of attractive places.

\subsubsection{Implications for urban planning}

There two implications for urban planning: firstly, the findings indicate that urban morphology as a public good has both positive and negative externalities on the property value. More specifically, a city's street network morphology distributes positive and negative agglomeration economies and diseconomies and other kinds of urban externality. A city's grid is therefore an important lever in influencing value within the urban economy. This is true because, as the thesis has shown, the pattern of externalities is capitalized in the land and property markets.

It requires prior evaluation of a street layout proposal before implementing infrastructure improvements to optimize externality effects. One of the key functions of urban government is to intervene in various ways to achieve such optimization. Governments provide urban public goods, otherwise, the market would fail to provide many of them because of the free-rider problem. Street networks are one important public good but as I have shown, they are much more than just one other good. A street improvement scheme provides direct benefits to travelers and the households and firms they belong to. But it may also have the effect of shifting the distribution of all the other aggregated and compounded public goods and values created by other such investments all across the city. It is vital for urban planners therefore to understand these kinds of systemic or global effects of local interventions. Space syntax and other such techniques can aid this understanding and the thesis has shown 
the possibility of measuring value changes throughout the city caused by changes to the urban spatial structure (Chiaradia et al. 2009).

Being more normative, we can define an important goal in urban planning as being to design and re-configure urban layout based on people's demand for different urban configurational patterns and different types of accessibility, bearing in mind that certain urban configurational features are associated with different preferences and social economic status. For example, many cities use regeneration developments to improve the attractiveness of areas. Although the building quality is improved, the project remains unattractive for high income people. One potential reason is that these regeneration projects fail to address the provision of high environmental quality and accessibility. Using network configurational analysis at different spatial scales, urban planners have a language and analytical tool for attempting optimize multiple objectives, including crucially, optimizing system-wide general accessibility (and land values) at the same time as optimizing local and sub-market-specific accessibility. In many ways this redefines the art and science of spatial planning.

\subsection{Limitation of these studies}

The limitations of this thesis relate to several issues. The first is the imperfections of data quality. The second issue is the violation of econometrics assumptions. The final issue is associated with the limitations of space syntax axial lines, and its handling of radii. The limitations provide directions for future research in analysis of urban morphology and housing market.

\subsubsection{Imperfections of data quality}

The data quality problems mean there are limitations on the use of models for 
estimation. For example, the Cardiff studies fail to account for all housing characteristics, such as the number of bedrooms and bathrooms, building age, school districts and distance to bus station. If data are not available for all housing characteristics, the unit price of housing cannot be estimated accurately from a simple hedonic house value function (McMillen 2003). Furthermore, in the Cardiff studies, the attributes of social economic status is at the aggregated level, so when applied to the disaggregated level, the attributes insufficient for capturing the housing price variance which in turn also leads to estimation bias. Finally, in the Chinese case study, the average selling price is an estimated price by developer based on expectations, thus it does not represent the average transaction price. Consequently, the estimated coefficients are not completely accurate.

\subsubsection{Econometrics issues}

The chapters using Cardiff as the case study also suffer from a number of econometrics issues, such spatial autocorrelation and heteroscedasticity. The basic assumptions underlying the regression model implies that the observations should be independent of one another, and that the variance of the error term does not correlate with the dependent variables. Although the space syntax variables provide more spatial information to reduce the spatial effect, the spatial autocorrelation still exists in the model of residual. Although these econometric issues do not necessarily influence the relationship between variables, they reduce the predictive power of the model. This can be solved by using some advanced estimations in order to adjust the model, such as multilevel specification, spatial lag or error model combined adjusted weighting. 


\subsubsection{Space syntax axial line and radii}

The creation of the space syntax line is another limitation. This thesis adopted Turner's suggestion (2007), creating the axial line map based on a digital road centre line map. However, due to the limitation of information on street networks, some spatial information cannot be interpreted in the axial line map, such as one way streets, which could affect the measurement of accessibility of the urban configuration.

The process of specifying space syntax radii seems arbitrary as there is a lack of precise definition to distinguish between each radii, particularly for integration and choice, and it is found that different radii could be highly correlated, thus it seems to be difficult to interpret social activity phenomena with each radii. Different researchers apply different sets of radii and the results are rarely coherent.

\subsection{Recommendation for future studies}

One direction to extend this dissertation is to understand how urban morphology as a public good can be priced accurately by people in each submarket, using advanced econometric models. For example, a longer period panel data for the Chinese study would allow us to observe how the change of urban configuration is associated with housing price volatility over time, particularly in each housing submarket or administration area. Furthermore, for the Cardiff study, empirical studies could also explore how different ranges of housing price are associated with different classes of urban morphology, or with preferences of different social economic classes in terms of the urban morphology. This could help government to address the socio-spatial segregation issues through a physical planning approach.

Secondly, the work in this dissertation could be extended to compare the externality of urban configuration on housing market in different local cultural contexts or places. 
The purpose of this direction would be to help both urban designers and planners to understand what kind of urban form has the lowest negative externality on the housing market. In addition, it could also help practitioners understand local people's demand with respect to urban form (see for example, Senior, Webster and Blank, 2004). For a stated preference modeling study of the demand for different urban morphologies, although that study did not use network morphology), which could offer some information for governments and developers on how to provide better and efficient service depending on demand. More generally, there is great scope for investigating the idea of optimizing net capitalized agglomeration economies via optimizing street network design.

Finally, the research can be extended to explore the relationship between urban morphology and other academic fields at a street level with high resolution data including information on land use, health and so on. For example, how is urban configuration associated with retail viability, use of urban public facilities, individual health (through the influence on exercise and congestion externalities), the speed of private urban services supply after the construction of new residential areas; and so on. At the most general level of urban planning, after a century of modern professional planning, there is still a lack of theoretical and practical guidance on how to distribute land use efficiently. 


\section{Reference}

Adair, A. et al. 1996. Hedonic modelling, housing submarkets and residential valuation. Journal of Property Research 13(1), pp. 67-83.

Adair, A. et al. 2000. House prices and accessibility: The testing of relationships within the Belfast urban area. Housing studies 15(5), pp. 699-716.

Alexander, C. 1964. Notes on the Synthesis of Form. Harvard Univ Pr.

Alexander, C. 1974 . A city is not a tree. utg.

Alkay, E. 2008. Housing submarkets in Istanbul. International Real Estate Review 11(1), p. $113^{*} \mathrm{C} 127$.

Allen, M. T. et al. 1995. Implicit pricing across residential rental submarkets. The Journal of Real Estate Finance and Economics 11(2), pp. 137-151.

Alonso, W. 1964. Location and land use: Toward a general theory of land rent. Publication of the Joint Center for Urban Studies. Harvard University Press.

Anderson, R. J. and Crocker, T. D. 1971. Air pollution and residential property values. Urban Studies 8(3), pp. 171-180.

Anderson, S. T. and West, S. E. 2006. Open space, residential property values, and spatial context. Regional science and urban economics 36(6), pp. 773-789.

Andersson, D. E. et al. 2010. Does high-speed rail accessibility influence residential property prices? Hedonic estimates from southern Taiwan. Journal of Transport Geography 18(1), pp. 166-174.

Anselin, L. 1988. Lagrange multiplier test diagnostics for spatial dependence and spatial heterogeneity. Geographical analysis 20(1), pp. 1-17.

Apergis, N. and Rezitis, A. 2003. Housing prices and macroeconomic factors in Greece: prospects within the EMU. Applied economics letters 10(12), pp. 799-804.

Atkin, R. 1978. Time as a pattern on a multi-dimensional structure. Journal of Social and Biological Structures 1(3), pp. 281-295.

Bacher, J. et al. 2004. SPSS twostep cluster: A first evaluation. Lehrstuhl für 
Soziologie.

Bajic, V. 1985. Housing(C) $\backslash$ market segmentation and demand for housing attributes: Some empirical findings. Real Estate Economics 13(1), pp. 58-75.

Ball, M. and Kirwan, R. 1977. Accessibility and supply constraints in the urban housing market. Urban Studies 14(1), pp. 11-32.

Ball, M. J. 1973. Recent empirical work on the determinants of relative house prices. Urban Studies 10(2), pp. 213-233.

Barros, A. P. et al. 2009. Transport Mobility at the University of Brasilia, Brazil.

Bartik, T. J. 1987. The estimation of demand parameters in hedonic price models. The journal of political economy 95(1), pp. 81-88.

Basu, S. and Thibodeau, T. G. 1998. Analysis of spatial autocorrelation in house prices. The Journal of Real Estate Finance and Economics 17(1), pp. 61-85.

Bateman, I. et al. 2001. The effect of road traffic on residential property values: a literature review and hedonic pricing study. Scottish Executive.

Bateman, I. and Executive, S. 2001. The effect of road traffic on residential property values: a literature review and hedonic pricing study. Scottish Executive.

Batty, M. 2001. Exploring isovist fields: space and shape in architectural and urban morphology. Environment and Planning B 28(1), pp. 123-150.

Batty, M. 2004a. A new theory of space syntax.

Batty, M. 2004b. A new theory of space syntax. London, UK: Centre for Advanced Spatial Analysis (UCL).

Batty, M. 2009. Accessibility: in search of a unified theory. Environment and Planning B: Planning and Design 36(2), pp. 191-194.

Batty, M. and Rana, S. 2002. Reformulating space syntax: the automatic definition and generation of axial lines and axial maps. Centre for Advanced Spatial Analysis Working Paper (58).

Batty, M. and Rana, S. 2004. The automatic definition and generation of axial lines and axial maps. Environment and Planning B 31(4), pp. 615-640.

Baumont, C. and Legros, D. 2009. Neighborhood Effects In Spatial Housing Value 
Models. The Case Of The Metropolitan Area Of Paris (1999).

Bavelas, A. 1948. A mathematical model for group structures. Human organization 7(3), pp. 16-30.

Bavelas, A. 1950. Communication patterns in task-oriented groups. Journal of the Acoustical Society of America.

Beckmann, M. J. 1973. Equilibrium models of residential land use. Regional and Urban Economics 3(4), pp. 361-368.

Bender, B. and Hwang, H. S. 1985. Hedonic housing price indices and secondary employment centers. Journal of Urban Economics 17(1), pp. 90-107.

Boarnet, M. G. 1994. The monocentric model and employment location. Journal of Urban Economics 36(1), pp. 79-97.

Bolitzer, B. and Netusil, N. R. 2000. The impact of open spaces on property values in Portland, Oregon. Journal of Environmental Management 59(3), pp. 185-193.

Bourassa, S. et al. 2005. Spatial dependence, housing submarkets, and house prices. Available at SSRN 771867.

Bourassa, S. C. et al. 1999. Defining housing submarkets. Journal of Housing Economics 8(2), pp. 160-183.

Bourassa, S. C. et al. 2003. Do housing submarkets really matter? Journal of Housing Economics 12(1), pp. 12-28.

Bourassa, S. C. and Peng, V. S. 1999. Hedonic prices and house numbers: The influence of feng shui. International Real Estate Review 2(1), pp. 79-93.

Bowen, W. M. et al. 2001. Theoretical and empirical considerations regarding space in hedonic housing price model applications. Growth and change 32(4), pp. 466-490.

Bowes, D. R. and Ihlanfeldt, K. R. 2001. Identifying the impacts of rail transit stations on residential property values. Journal of Urban Economics 50(1), pp. 1-25.

Brasington, D. M. and Hite, D. 2005. Demand for environmental quality: a spatial hedonic analysis. Regional science and urban economics 35(1), pp. 57-82.

Brown, G. M. 1999. Design and value: spatial form and the economic failure of a mall. Journal of Real Estate Research 17(2), pp. 189-225. 
Bryk, A. S. and Lee, V. E. 1992. Is politics the problem and markets the answer? An essay review of Politics, Markets, and America's Schools. Economics of Education Review 11(4), pp. 439-451.

Button, K. and Taylor, S. 2000. International air transportation and economic development. Journal of Air Transport Management 6(4), pp. 209-222.

Can, A. 1990. The measurement of neighborhood dynamics in urban house prices. Economic geography, pp. 254-272.

Capozza, D. R. and Helsley, R. W. 1989. The fundamentals of land prices and urban growth. Journal of Urban Economics 26(3), pp. 295-306.

Carvalho, R. and Penn, A. 2004. Scaling and universality in the micro-structure of urban space. Physica A: Statistical Mechanics and its Applications 332, pp. 539-547.

Case, B. et al. 2006. The Impact of Environmental Contamination on Condo Prices: A Hybrid Repeat - Sale/Hedonic Approach. Real Estate Economics 34(1), pp. 77-107.

Case, B. and Quigley, J. M. 1991. The dynamics of real estate prices. The Review of Economics and Statistics, pp. 50-58.

Case, K. E. et al. 2005. Comparing Wealth Effects: The Stock Market versus the Housing Market.

Cassel, E. and Mendelsohn, R. 1985. The choice of functional forms for hedonic price equations: Comment. Journal of Urban Economics 18(2), pp. 135-142.

Chambers, D. N. 1992. The racial housing price differential and racially transitional neighborhoods. Journal of Urban Economics 32(2), pp. 214-232.

Chau, K. W. et al. 2001. The pricing of 'luckiness' in the apartment market. Journal of Real Estate Literature 9(1), pp. 29-40.

Chen, X. and Gao, X. 1993. Urban economic reform and public-housing investment in China. Urban Affairs Review 29(1), pp. 117-145.

Cheshire, P. C. and Sheppard, S. 1997. The welfare economics of land use regulation. London School of Economics, Department of Geography.

Chiaradia, A. et al. 2009. Residential property value patterns in London: space syntax spatial analysis.

Chiu, T. et al. eds. 2001. A robust and scalable clustering algorithm for mixed type 
attributes in large database environment. Proceedings of the seventh ACM SIGKDD international conference on knowledge discovery and data mining. ACM.

Chow, G. C. 1960. Tests of equality between sets of coefficients in two linear regressions. Econometrica: Journal of the Econometric Society, pp. 591-605.

Christopher, A. ed. 1965. A city is not a tree.

Clapp, J. M. and Giaccotto, C. 1992. Estimating price indices for residential property: a comparison of repeat sales and assessed value methods. Journal of the American Statistical Association, pp. 300-306.

Collinge, S. K. 1996. Ecological consequences of habitat fragmentation: Implications for landscape architecture and planning. Landscape and Urban Planning 36(1), pp. $59-77$.

Corbusier, L. and Guiton, J. 1981. The ideas of Le Corbusier on architecture and urban planning. George Braziller.

Cropper, M. L. et al. 1988. On the Choice of Funtional Form for Hedonic Price Functions. The Review of Economics and Statistics, pp. 668-675.

Croxford, B. et al. 1996. Spatial distribution of urban pollution: civilizing urban traffic. Science of the total environment 189, pp. 3-9.

Cubbin, J. S. 1970. A hedonic approach to some aspects of the Coventry housing market. University of Warwick, Department of Economics.

Dale-Johnson, D. 1982. An alternative approach to housing market segmentation using hedonic price data. Journal of Urban Economics 11(3), pp. 311-332.

Dalton, N. S. et al. 2003. To tame a TIGER one has to know its nature: extending weighted angular integration analysis to the descriptionof GIS road-centerline data for large scale urban analysis.

Day, B. 2003. Submarket identification in property markets: a hedonic housing price model for Glasgow. University of East Anglia England: The Centre for Social and Economic Research on the Global Environment School of Environmental Sciences.

Day, B. et al. 2007. Beyond implicit prices: recovering theoretically consistent and transferable values for noise avoidance from a hedonic property price model. Environmental and resource economics 37(1), pp. 211-232.

Debrezion, G. et al. 2006. The impact of rail transport on real estate prices: an 
empirical analysis of the Dutch housing market.

Des Rosiers, F. et al. 1999. Environment and value Does drinking water quality affect house prices? Journal of Property Investment \& Finance 17(5), pp. 444-463.

Des Rosiers, F. et al. 2000. Sorting out access and neighbourhood factors in hedonic price modelling. Journal of Property Investment \& Finance 18(3), pp. 291-315.

Desyllas, J. 2000. The relationship between urban street configuration and office rent patterns in Berlin. Architecture. London, University College London. PhD 345.

Dewees, D. N. 1976. The effect of a subway on residential property values in Toronto. Journal of Urban Economics 3(4), pp. 357-369.

Dhrymes, P. J. 1971. Distributed lags: problems of estimation and formulation. Holden-Day San Francisco.

Diewert, W. E. ed. 2003. Hedonic regressions: a review of some unresolved issues.

Dougherty, J. et al. 2009. School Choice in Suburbia: Test Scores, Race, and Housing Markets. American Journal of Education 115(4), pp. 523-548.

Downes, T. A. and Zabel, J. E. 2002. The impact of school characteristics on house prices: Chicago 1987-1991. Journal of Urban Economics 52(1), pp. 1-25.

Dubin, R. A. and Goodman, A. C. 1982. Valuation of education and crime neighborhood characteristics through hedonic housing prices. Population \& Environment 5(3), pp. 166-181.

Dubin, R. A. and Sung, C. H. 1987. Spatial variation in the price of housing: rent gradients in non-monocentric cities. Urban Studies 24(3), pp. 193-204.

Dubin, R. A. and Sung, C. H. 1990. Specification of hedonic regressions: non-nested tests on measures of neighborhood quality. Journal of Urban Economics 27(1), pp. 97-110.

Dunse, N. and Jones, C. 1998. A hedonic price model of office rents. Journal of Property Valuation and Investment 16(3), pp. 297-312.

Enström, R. and Netzell, O. 2008. Can space syntax help us in understanding the intraurban office rent pattern? Accessibility and rents in downtown Stockholm. The Journal of Real Estate Finance and Economics 36(3), pp. 289-305.

Epp, D. J. and Al-Ani, K. S. 1979. The effect of water quality on rural nonfarm 
residential property values. American Journal of Agricultural Economics 61(3), pp. 529-534.

Epple, D. 1987. Hedonic prices and implicit markets: estimating demand and supply functions for differentiated products. The journal of political economy 95(1), pp. 59-80.

Evans, A. W. 1985. Urban economics: an introduction. Basil Blackwell Oxford.

Farber, S. 1998. Undesirable facilities and property values: a summary of empirical studies. Ecological Economics 24(1), pp. 1-14.

Feitelson, E. 1993. An hierarchical approach to the segmentation of residential demand: theory and application. Environment and Planning A 25, pp. 553-553.

Fletcher, M. et al. 2000. Heteroscedasticity in hedonic house price models. Journal of Property Research 17(2), pp. 93-108.

Follain, J. R. and Jimenez, E. 1985. Estimating the demand for housing characteristics: a survey and critique. Regional science and urban economics 15(1), pp. 77-107.

Freeman, A. M. I. 1979. Benefits of environmental improvement: theory and practice. p. Medium: X; Size: Pages: 282.

Freeman III, A. M. 1979a. Benefits of environmental improvement: theory and practice.

Freeman III, A. M. 1979b. Hedonic prices, property values and measuring environmental benefits: a survey of the issues. The Scandinavian Journal of Economics, pp. 154-173.

Freeman III, A. M. 1993. Nonuse values in natural resource damage assessment. Valuing Natural Assets. The Economics of Natural Resource Damage Assessment. Washington DC: Resources for the Future, pp. 264-306.

Gabriel, S. A. and Wolch, J. R. 1984a. Spillover effects of human service facilities in a racially segmented housing market. Journal of Urban Economics 16(3), pp. 339-350.

Gabriel, S. A. and Wolch, J. R. 1984b. Spillover effects of human service facilities in a racially segmented housing market* 1. Journal of Urban Economics 16(3), pp. 339-350.

Gans, R. 1963. Common sense in teaching reading. Bobbs-Merrill. 
Garrett, T. A. 2003. Aggregated versus disaggregated data in regression analysis: implications for inference. Economics Letters 81(1), pp. 61-65.

Gatzlaff, D. H. and Smith, M. T. 1993. The impact of the Miami Metrorail on the value of residences near station locations. Land Economics, pp. 54-66.

Gibbons, S. and Machin, S. 2003. Valuing English primary schools. Journal of Urban Economics 53(2), pp. 197-219.

Gibbons, S. and Machin, S. 2005. Valuing rail access using transport innovations. Journal of Urban Economics 57(1), pp. 148-169.

Gillard, Q. 1981. The effect of environmental amenities on house values: the example of a view lot. The Professional Geographer 33(2), pp. 216-220.

Gillen, K. et al. 2001. Anisotropic autocorrelation in house prices. The Journal of Real Estate Finance and Economics 23(1), pp. 5-30.

Goodchild, M. F. 1986. Spatial autocorrelation. Geo Books Norwich.

Goodman, A. C. 1981. HOUSING SUBMARKETS WITHIN URBAN AREAS: DEFINITIONS AND EVIDENCE*. Journal of regional science 21(2), pp. 175-185.

Goodman, A. C. and Thibodeau, T. G. 1998. Housing Market Segmentation. Journal of Housing Economics 7(2), pp. 121-143.

Goodman, A. C. and Thibodeau, T. G. 2003. Housing market segmentation and hedonic prediction accuracy. Journal of Housing Economics 12(3), pp. 181-201.

Goodman, A. C. and Thibodeau, T. G. 2007. The spatial proximity of metropolitan area housing submarkets. Real Estate Economics 35(2), pp. 209-232.

Graves, P. E. et al. 1988. The Robustness of Hedonic Price Estimation. Land Economics 64(3), pp. 220-233.

GREAVES, M. 1985. The determinants of residential values: the hierarchical and statistical approaches. Journal of Property Valuation and Investment 3(1), pp. 5-23.

Greene, W. H. 2008. The econometric approach to efficiency analysis. The measurement of productive efficiency and productivity growth, pp. 92-250.

Griliches, Z. 1961. Staff papers 3. hedonic price indexes for automobiles: An econometric of quality change. NBER. 
Griliches, Z. 1996. The discovery of the residual: a historical note. Journal of Economic Literature 34(3), pp. 1324-1330.

Gujarati, D. N.,(2003), Basic econometrics. New York: MeGraw-Hill, pp. 363-369.

Haggett, P. and Chorley, R. J. 1969. Network analysis in geography. Edward Arnold (London).

Halvorsen, R. and Pollakowski, H. O. 1981. Choice of functional form for hedonic price equations. Journal of Urban Economics 10(1), pp. 37-49.

Hamilton, B. W. 1989. Wasteful commuting again. The journal of political economy 97(6), pp. 1497-1504.

Hamilton, B. W. and Röell, A. 1982. Wasteful commuting. The journal of political economy, pp. 1035-1053.

Hancock, K. 1991. The determination of housing submarkets: case studies using Scottish data. unpublished paper, Centre for Housing Research, University of Glasgow, Glasgow.

Handy, S. L. and Niemeier, D. A. 1997. Measuring accessibility: an exploration of issues and alternatives. Environment and Planning A 29, pp. 1175-1194.

Hansen, W. G. 1959. How accessibility shapes land use. Journal of the American Institute of Planners 25(2), pp. 73-76.

Harding, J. P. et al. 2003. Estimating bargaining effects in hedonic models: evidence from the housing market. Real Estate Economics 31(4), pp. 601-622.

Harris, D. R. 1999. " Property Values Drop When Blacks Move in, Because...": Racial and Socioeconomic Determinants of Neighborhood Desirability. American sociological review, pp. 461-479.

Harter-Dreiman, M. 2004. Drawing inferences about housing supply elasticity from house price responses to income shocks. Journal of Urban Economics 55(2), pp. 316-337.

Haurin, D. R. and Brasington, D. 1996. School quality and real house prices: Inter-and intrametropolitan effects. Journal of Housing Economics 5(4), pp. 351-368.

Hefner, F. House Price Impacts of School District Choice1 John A. Kilpatrick Mundy and Associates Suite 200, 1109 First Avenue Seattle, Washington 98101. 
Heikkila, E. et al. 1989. What happened to the CBD-distance gradient? Land values in a policentric city. Environment and Planning A 21(2), pp. 221-232.

Hendry, D. F. 1995. Dynamic econometrics. Oxford University Press, USA.

Henneberry, J. 1998. Transport investment and house prices. Journal of Property Valuation and Investment 16(2), pp. 144-158.

Hillier, B. 1996. Space is the Machine. Cambridge University Press Cambridge.

Hillier, B. 2004. Can streets be made safe? Urban Design International 9(1), pp. $31-45$.

Hillier, B. and Hanson, J. 1984. The social logic of space. Cambridge University Press Cambridge.

Hillier, B. et al. 1987. IDEAS ARE IN THINGS-AN APPLICATION OF THE SPACE SYNTAX METHOD TO DISCOVERING HOUSE GENOTYPES. ENVIRON PLANN B 14(4), pp. 363-385.

Hillier, B. and Iida, S. 2005. Network and psychological effects in urban movement. Spatial Information Theory, pp. 475-490.

Hillier, B. and Penn, A. 2004. Rejoinder to Carlo Ratti. ENVIRONMENT AND PLANNING B-PLANNING \& DESIGN 31(4), pp. 501-511.

Hillier, B. et al. 1993a. Natural movement-or, configuration and attraction in urban pedestrian movement. ENVIRON PLANN B 20(1), pp. 29-66.

Hillier, B. et al. 1993b. Natural movement: or, configuration and attraction in urban pedestrian movement. Environment and Planning B: Planning and Design 20(1), pp. 29-66.

Hoch, I. and Waddell, P. 1993. Apartment rents: another challenge to the monocentric model. Geographical Analysis 25(1), pp. 20-34.

Holly, S. and Jones, N. 1997. House prices since the 1940s: cointegration, demography and asymmetries. Economic Modelling 14(4), pp. 549-565.

Hort, K. 1998. The determinants of urban house price fluctuations in Sweden 1968-1994. Journal of Housing Economics 7(2), pp. 93-120.

Howard, E. 1965. Garden Cities of Tomorrow. 1898. Reprint. Cambridge, Mass.: 
MIT Press.

Hoyt, H. 1939. The structure and growth of residential neighborhoods in American cities.

Hsiao, C. 2003. Analysis of panel data. Cambridge Univ Pr.

Hulten, C. 2005. Price hedonics: a critical review. Economic Policy Review 9(3).

Hurd, R. M. 1903. Principles of city land values. The Record and Guide.

Hwang, S. and Thill, J. C. 2009. Delineating urban housing submarkets with fuzzy clustering. Environment and Planning B: Planning and Design 36(5), pp. 865-882.

Iacono, M. and Levinson, D. 2011. Accessibility Dynamics and Location Premia: Do Land Values Follow Accessibility Changes? Working Papers.

Ioannides, Y. M. 2002. Residential neighborhood effects. Regional Science and Urban Economics 32(2), pp. 145-165.

Jackson, J. R. 1979. Intraurban variation in the price of housing. Journal of Urban Economics 6(4), pp. 464-479.

Jacobs, J. 1961. The death and life of great American cities. Vintage.

Jiang, B. and Claramunt, C. 2002. Integration of space syntax into GIS: new perspectives for urban morphology. Transactions in GIS 6(3), pp. 295-309.

Jiang, B. and Claramunt, C. 2004. Topological analysis of urban street networks. Environment and Planning B 31(1), pp. 151-162.

Jiang, B. and Liu, X. 2009. AxialGen: a research prototype for automatically generating the axial map. Arxiv preprint arXiv:0902.0465.

Johnson, M. S. and Ragas, W. R. 1987. CBD land values and multiple externalities. Land Economics 63(4), pp. 337-347.

Jones, C. et al. 2003. Structural change in a local urban housing market. Environment and Planning A 35(7), pp. 1315-1326.

Jones, C. et al. 2004. Intra-urban migration and housing submarkets: theory and evidence. Housing Studies 19(2), pp. 269-283.

Jud, G. D. et al. 2002. The impact of information technology on real estate licensee 
income. Journal of Real Estate Practice and Education 5(1), pp. 1-16.

Kain, J. F. and Quigley, J. M. 1970. Measuring the value of housing quality. Journal of the American Statistical Association 65(330), pp. 532-548.

Kauko, T. et al. 2002. Capturing housing market segmentation: an alternative approach based on neural network modelling. Housing Studies 17(6), pp. 875-894.

Kholdy, S. and Sohrabian, A. 1998. Economic fundamentals and housing market. Applied economics letters 5(7), pp. 459-461.

Kiel, K. A. 1995. Measuring the impact of the discovery and cleaning of identified hazardous waste sites on house values. Land Economics, pp. 428-435.

King, A. T. and Mieszkowski, P. 1973. Racial discrimination, segregation, and the price of housing. The journal of political economy, pp. 590-606.

Klevmarken, N. A. 1989. Introduction. European Economic Review 33(2-3), pp. 523-529.

Kohlhase, J. E. 1991. The impact of toxic waste sites on housing values. Journal of Urban Economics 30(1), pp. 1-26.

Krüger, M. 1979. An approach to built-form connectivity at an urban scale: variations of connectivity and adjacency measures amongst zones and other related topics. Environment and Planning B 6(3), pp. 305-320.

Kwan, M. P. 1998. Space - time and integral measures of individual accessibility: a comparative analysis using a point - based framework. Geographical analysis 30(3), pp. 191-216.

Lake, I. R. et al. 2000. Using GIS and large-scale digital data to implement hedonic pricing studies. International Journal of Geographical Information Science 14(6), pp. 521-541.

Lancaster, K. J. 1966. A new approach to consumer theory. The journal of political economy 74(2), pp. 132-157.

Landau, U. et al. 1981. The effect of temporal constraints on household travel behavior. Environment and Planning A 13(4), pp. 435-448.

Larkham, P. J. 2006. The study of urban form in Great Britain. Urban Morphology 10(2), p. 117. 
Lawrence, D. L. and Low, S. M. 1990. The built environment and spatial form. Annual review of anthropology 19, pp. 453-505.

Leach, E. 1978. Culture and reality. Psychological Medicine 8(4), pp. 555-564.

Leavitt, H. J. 1951. Some effects of certain communication patterns on group performance. The Journal of Abnormal and Social Psychology 46(1), p. 38.

Linneman, P. 1980. Some empirical results on the nature of the hedonic price function for the urban housing market. Journal of Urban Economics 8(1), pp. 47-68.

Lutzenhiser, M. and Netusil, N. R. 2001. The effect of open spaces on a home's sale price. Contemporary Economic Policy 19(3), pp. 291-298.

Lynch, K. 1960. The city image and its elements. The image of the city, pp. 46-90.

Maclennan, D. et al. 1987a. Housing choice and the structure of urban housing markets. Between State and Market Housing in the Post-industrial Era, p. 26"C52.

Maclennan, D. et al. 1987b. Housing choice and the structure of urban housing markets. Between State and Market Housing in the Post-industrial Era, pp. 27-45.

Maclennan, D. and Tu, Y. 1996. Economic perspectives on the structure of local housing systems. Housing Studies 11(3), pp. 387-406.

Maddison, D. 2001. The amenity value of the global climate. Earthscan.

March, L. and Steadman, P. 1971. The Geometry of Environments: An Introduction to Spatial Organizaiton in Design. Methuen \& Company Limited.

Martin, L. and March, L. 1972. Urban space and structures. Cambridge University Press.

Matthews, J. W. and Turnbull, G. K. 2007. Neighborhood street layout and property value: the interaction of accessibility and land use mix. The Journal of Real Estate Finance and Economics 35(2), pp. 111-141.

McConnell, V. and Walls, M. A. 2005. The value of open space: Evidence from studies of nonmarket benefits. Resources for the Future.

McDonald, J. F. 1987. The identification of urban employment subcenters. Journal of Urban Economics 21(2), pp. 242-258.

McLeod, P. B. 1984. The demand for local amenity: an hedonic price analysis. 
Environment and Planning A 16(3), pp. 389-400.

McMillen, D. P. 2001. Nonparametric employment subcenter identification. Journal of Urban Economics 50(3), pp. 448-473.

McMillen, D. P. 2003. The return of centralization to Chicago: using repeat sales to identify changes in house price distance gradients. Regional Science and Urban Economics 33(3), pp. 287-304.

McMillen, D. P. 2004. Airport expansions and property values: the case of Chicago O'Hare Airport. Journal of Urban Economics 55(3), pp. 627-640.

McMillen, D. P. and McDonald, J. 2004. Reaction of house prices to a new rapid transit line: Chicago's midway line, 1983-1999. Real Estate Economics 32(3), pp. 463-486.

McMillen, D. P. and Thorsnes, P. 2006. Housing Renovations and the Quantile Repeat - Sales Price Index. Real Estate Economics 34(4), pp. 567-584.

Meen, D. and Meen, G. 2003. Social behaviour as a basis for modelling the urban housing market: a review. Urban Studies 40(5-6), p. 917.

Meese, R. and Wallace, N. 2003. House price dynamics and market fundamentals: the Parisian housing market. Urban Studies 40(5-6), p. 1027.

Mendelsohn, R. et al. 1992. Measuring hazardous waste damages with panel models. Journal of Environmental Economics and Management 22(3), pp. 259-271.

Michael, H. J. et al. 1996. Water quality affects property prices: A case study of selected Maine lakes. Maine Agricultural and Forest Experiment Station, University of Maine.

Michaels, R. G. and Smith, V. K. 1990. Market segmentation and valuing amenities with hedonic models: The case of hazardous waste sites. Journal of Urban Economics 28(2), pp. 223-242.

Michelson, W. 1977. Environmental choice, human behavior, and residential satisfaction. Taylor \& Francis.

Mieszkowski, P. and Zodrow, G. R. 1989. Taxation and the Tiebout model: the differential effects of head taxes, taxes on land rents, and property taxes. Journal of Economic Literature 27(3), pp. 1098-1146.

Mills, E. S. 1972. Markets and efficient resource allocation in urban areas. The 
Swedish Journal of Economics, pp. 100-113.

Min, K. M. et al. eds. 2007. The effect of spatial configuration on land use and land value in Seoul.

Monn, C. 2001. Exposure assessment of air pollutants: a review on spatial heterogeneity and indoor/outdoor/personal exposure to suspended particulate matter, nitrogen dioxide and ozone. Atmospheric Environment 35(1), pp. 1-32.

Morris, E. W. et al. 1972. The measurement of housing quality. Land Economics, pp. 383-387.

Moudon, A. V. 1997. Urban morphology as an emerging interdisciplinary field. Urban Morphology 1(1), pp. 3-10.

Munoz-Raskin, R. 2010. Walking accessibility to bus rapid transit: Does it affect property values? The case of Bogotá, Colombia. Transport Policy 17(2), pp. 72-84.

Munro, M. 1986. Testing for segmentation in the private housing market in Glasgow. Centre for Housing Research, Discussion Paper No 8.

Muratori, S. 1959. Studi per una operante storia urbana di Venezia. Istituto poligrafico dello Stato, Libreria dello Stato.

Muratori, S. and di Storia Urbanistica, C. S. 1963. Studi per una operante storia urbana di Roma. Consiglio nazionale delle ricerche.

Murdoch, J. C. and Thayer, M. A. 1988. Hedonic price estimation of variable urban air quality. Journal of Environmental Economics and Management 15(2), pp. 143-146.

Muth, R. F. 1969. CITIES AND HOUSING; THE SPATIAL PATTERN OF URBAN RESIDENTIAL LAND USE.

Nelson, J. P. 1982. Highway noise and property values: a survey of recent evidence. Journal of Transport Economics and Policy, pp. 117-138.

Nes, A. and Rueb, L. 2009. Spatial Behaviour in Dutch Dwelling Areas.

Neumann, B. C. 2005. Is All Open Space Created Equal? A Hedonic Application within a Data-Rich GIS Environment.

Nghiep, N. and Al, C. 2001. Predicting housing value: A comparison of multiple regression analysis and artificial neural networks. Journal of Real Estate Research 
22(3), pp. 313-336.

Niedercorn, J. H. and Ammari, N. S. 1987. New evidence on the specification and performance of neoclassical gravity models in the study of urban transportation. The Annals of Regional Science 21(1), pp. 56-64.

Niemeier, D. A. 1997. Accessibility: an evaluation using consumer welfare. Transportation 24(4), pp. 377-396.

Noonan, D. S. 2007. Finding an impact of preservation policies: Price effects of historic landmarks on attached homes in chicago, 1990-1999. Economic development quarterly 21(1), pp. 17-33.

Nowak, D. and McPherson, E. 1993. Quantifying the impact of trees: The Chicago urban forest climate project. Unasylva 44.

Oates, W. E. 1969. The effects of property taxes and local public spending on property values: An empirical study of tax capitalization and the Tiebout hypothesis. The journal of political economy 77(6), pp. 957-971.

Openshaw, S. 1973. Insoluble problems in shopping model calibration when the trip pattern is not known. Regional Studies 7(4), pp. 367-371.

Orford, S. 1999. Valuing the built environment. G1S and house.

Orford, S. 2000. Modelling spatial structures in local housing market dynamics: a multilevel perspective. Urban Studies 37(9), p. 1643.

Orford, S. 2002. Valuing locational externalities: a GIS and multilevel modelling approach. Environment and Planning B 29(1), pp. 105-128.

Osland, L. and Thorsen, I. 2008. Effects on housing prices of urban attraction and labor-market accessibility. Environment and planning. A 40(10), p. 2490.

Osman, K. M. and Suliman, M. 1996. Spatial and cultural dimensions of the houses of Omdurman, Sudan. Human Relations 49(4), pp. 395-428.

Palm, R. 1978. Spatial Segmentation of the Urban Housing Market. Economic Geography 54(3), pp. 210-221.

Penn, A. 2003. Space Syntax and Spatial Cognition or Why the axial line? Environment and Behavior 35(1), pp. 30-65.

Penn, A. et al. 1997. Intelligent Architecture. Virtual reality, training's future?: 
perspectives on virtual reality and related emerging technologies 6, p. 121.

Penn, A. et al. 1998. Configurational modelling of urban movement networks. Environment and Planning B: Planning and Design 25(1), pp. 59-84.

Peponis, J. et al. 1998. On the generation of linear representations of spatial configuration. Environment and Planning B 25, pp. 559-576.

Polinsky, A. M. and Rubinfeld, D. L. 1977. Property Values and the Benefits of Environmental Improvements: Theory and Measurement. Public economics and the quality of life, p. 154.

Polinsky, A. M. and Shavell, S. 1982. Pigouvian taxation with administrative costs. Journal of Public Economics 19(3), pp. 385-394.

Pollakowski, H. O. 1982. Urban housing markets and residential location. Lexington books Lexington, MA.

Porta, S. et al. 2006. The network analysis of urban streets: a dual approach. Physica A: Statistical Mechanics and its Applications 369(2), pp. 853-866.

Poudyal, N. C. et al. 2009. A hedonic analysis of the demand for and benefits of urban recreation parks. Land Use Policy 26(4), pp. 975-983.

Powe, N. et al. 1995. Valuation of urban amenities using an hedonic price model. Journal of Property Research 12(2), pp. 137-147.

Quigley, J. M. 1995. A simple hybrid model for estimating real estate price indexes. Journal of Housing Economics 4(1), pp. 1-12.

Rapkin, C. 1953. Housing market analysis: a study of theory and methods. Housing and Home Finance Agency, Office of the Administrator, Division of Housing Research.

Ratti, C. 2004. Urban texture and space syntax: some inconsistencies. Environment and Planning B: Planning and Design 31(4), pp. 487-499.

Redfearn, C. L. 2007. The topography of metropolitan employment: Identifying centers of employment in a polycentric urban area. Journal of Urban Economics 61(3), pp. 519-541.

Richardson, H. W. 1977. On the possibility of positive rent gradients. Journal of Urban Economics 4(1), pp. 60-68. 
Richardson, H. W. et al. 1990. Residential property values, the CBD, and multiple nodes: further analysis. Environment and Planning A 22(6), pp. 829-833.

Riddel, M. 2004. Housing-market disequilibrium: an examination of housing-market price and stock dynamics 1967-1998. Journal of Housing Economics 13(2), pp. 120-135.

Ridker, R. G. and Henning, J. A. 1967. The determinants of residential property values with special reference to air pollution. The Review of Economics and Statistics 49(2), pp. 246-257.

Rolon, A. 2005. Value Capture as a Potential Source for Funding Transportation Projects in the City of Querétaro, Mexico.

Rosen, S. 1974. Hedonic prices and implicit markets: product differentiation in pure competition. The journal of political economy, pp. 34-55.

Rothenberg, J. et al. 1991. The maze of urban housing markets: Theory, evidence, and policy. University of Chicago Press.

Schafer, R. 1979. Racial discrimination in the Boston housing market. Journal of Urban Economics 6(2), pp. 176-196.

Schipper, Y. et al. 1998. Why do aircraft noise value estimates differ? A meta-analysis. Journal of Air Transport Management 4(2), pp. 117-124.

Schnare, A. B. and Struyk, R. J. 1976. Segmentation in urban housing markets. Journal of Urban Economics 3(2), pp. 146-166.

Shaw, M. E. 1954. Group structure and the behavior of individuals in small groups. The Journal of Psychology 38(1), pp. 139-149.

Shaw, M. E. 1964. Communication networks. Advances in experimental social psychology 1, pp. 111-147.

Shen, Q. 1998. Spatial technologies, accessibility, and the social construction of urban space. Computers, environment and urban systems 22(5), pp. 447-464.

Sheppard, J. A. 1974. Metrological analysis of regular village plans in Yorkshire. The Agricultural History Review, pp. 118-135.

Sheppard, S. 1999. Hedonic analysis of housing markets. Handbook of regional and urban economics 3, pp. 1595-1635. 
Shimbel, A. 1953. Structural parameters of communication networks. Bulletin of Mathematical Biology 15(4), pp. 501-507.

Sima, Y. and Zhang, D. eds. 2009a. Comparative Precedents on the Study of Urban Morphology. 2009). Proceedings of the 7th International Space Syntax Symposium. Stockholm: KTH.

Sima, Y. and Zhang, D. eds. 2009b. Comparative Precedents on the Study of Urban Morphology.

Sirmans, G. S. et al. 2006. The value of housing characteristics: a meta analysis. The Journal of Real Estate Finance and Economics 33(3), pp. 215-240.

Sirmans, S. G. et al. 2005. The composition of hedonic pricing models. Journal of Real Estate Literature 13(1), pp. 1-44.

Slater, T. 1981. The analysis of burgage patterns in medieval towns. Area, pp. 211-216.

Slater, T. 1988. English medieval town planning. Urban historical geography: recent progress in Britain and Germany, pp. 93-105.

Small, K. A. and Song, S. 1992. " Wasteful" commuting: a resolution. The journal of political economy 100(4), pp. 888-898.

Smith, V. K. and Huang, J. C. 1995. Can markets value air quality? A meta-analysis of hedonic property value models. Journal of political economy, pp. 209-227.

So, H. et al. 1997. Estimating the influence of transport on house prices: evidence from Hong Kong. Journal of Property Valuation and Investment 15(1), pp. 40-47.

Song, Y. and Sohn, J. 2007. Valuing spatial accessibility to retailing: A case study of the single family housing market in Hillsboro, Oregon. Journal of Retailing and Consumer Services 14(4), pp. 279-288.

Sonstelie, J. C. and Portney, P. R. 1980. Gross rents and market values: Testing the implications of Tiebout's hypothesis* 1. Journal of Urban Economics 7(1), pp. 102-118.

Srinivasan, S. and Ferreira, J. 2002. Travel behavior at the household level: understanding linkages with residential choice. Transportation Research Part D: Transport and Environment 7(3), pp. 225-242.

Srour, I. M. et al. 2002. Accessibility indices: connection to residential land prices and 
location choices. Transportation Research Record: Journal of the Transportation Research Board 1805(-1), pp. 25-34.

Steadman, M. and March, L. 1971. The geometry of the environment: an introduction to spatial organization in design. Methuen London.

Steadman, P. 1983. Architectural morphology: an introduction to the geometry of building plans. Pion Londres.

Steadman, P. 2004. Developments in space syntax. Environment and Planning B: Planning and Design 31(4), pp. 483-486.

Stegman, M. A. 1969. Accessibility models and residential location. Journal of the American Institute of Planners 35(1), pp. 22-29.

Steinnes, D. N. 1977. Causality and intraurban location. Journal of Urban Economics 4(1), pp. 69-79.

Steinnes, D. N. 1982. Do'people follow jobs' or do'jobs follow people'? A causality issue in urban economics. Urban Studies 19(2), pp. 187-192.

Steinnes, D. N. 1992. Measuring the economic value of water quality. The Annals of Regional Science 26(2), pp. 171-176.

Stern, D. 1992. Explaining UK house price inflation 1971-89. Applied Economics 24(12), pp. 1327-1333.

Stevenson, S. 2004. House price diffusion and inter - regional and cross - border house price dynamics. Journal of Property Research 21(4), pp. 301-320.

Stewart, J. Q. 1947. Empirical mathematical rules concerning the distribution and equilibrium of population. Geographical Review 37(3), pp. 461-485.

Straszheim, M. 1974. Hedonic estimation of housing market prices: A further comment. The Review of Economics and Statistics 56(3), pp. 404-406.

Straszheim, M. R. 1975. An econometric analysis of the urban housing market. NBER Books.

Takala, B. B. K. and Barot, B. 1998. House prices and inflation: a cointegration analysis for Finland and Sweden. Bank of Finland Discussion Papers 12, p. 98.

Tang, F. 1975. DETECTION AND ESTIMATION OF TRANSPORTATION IMPACT WITH MODELS OF SUBURBAN RESIDENTIAL PROPERTY SALES 


\section{PRICES.}

Thorsnes, P. 2002. The value of a suburban forest preserve: Estimates from sales of vacant residential building lots. Land Economics 78(3), pp. 426-441.

Tian, L. 2006. Impacts of transport projects on residential property values in China: Evidence from two projects in Guangzhou. Journal of Property Research 23(4), pp. 347-365.

Tobler, W. R. 1970. A computer movie simulating urban growth in the Detroit region. Economic geography 46, pp. 234-240.

Tse, R. Y. C. and Love, P. E. D. 2000. Measuring residential property values in Hong Kong. Property Management 18(5), pp. 366-374.

Tu, Y. 1997. The local housing sub-market structure and its properties. Urban Studies 34(2), pp. 337-353.

Tu, Y. et al. 2007. Spatial autocorrelations and urban housing market segmentation. The Journal of Real Estate Finance and Economics 34(3), pp. 385-406.

Turner, A. 2007. From axial to road-centre lines: a new representation for space syntax and a new model of route choice for transport network analysis. Environment and Planning B: Planning and Design 34(3), pp. 539-555.

Turner, A. et al. 2005. An algorithmic definition of the axial map. Environment and Planning B: Planning and Design 32(3), pp. 425-444.

Tyrväinen, L. 1997. The amenity value of the urban forest: an application of the hedonic pricing method. Landscape and Urban planning 37(3), pp. 211-222.

van der Vlist, A. J. et al. 2011. Immigration and urban housing market dynamics: the case of Haifa. The Annals of Regional Science 47(3), pp. 585-598.

Vaughan, L. et al. 2005. Space and exclusion: does urban morphology play a part in social deprivation? Area 37(4), pp. 402-412.

Vaughan, L. and Penn, A. 2006. Jewish immigrant settlement patterns in Manchester and Leeds 1881. Urban studies 43(3), p. 653.

Visser, P. et al. 2008. Residential environment and spatial variation in house prices in the Netherlands. Tijdschrift voor economische en sociale geografie 99(3), pp. 348-360. 
Wachs, M. and Kumagai, T. G. 1973. Physical accessibility as a social indicator. Socio-Economic Planning Sciences 7(5), pp. 437-456.

Waddell, P. et al. 1993. Housing price gradients: The intersection of space and built form. Geographical Analysis 25(1), pp. 5-19.

Wang, F. 2001. Explaining intraurban variations of commuting by job proximity and workers' characteristics. Environment and Planning B 28(2), pp. 169-182.

Wang, Y. P. and Murie, A. 2000. Social and spatial implications of housing reform in China. International Journal of Urban and Regional Research 24(2), pp. 397-417.

Ward, M. D. and Gleditsch, K. S. 2008. Spatial regression models. Sage Publications, Incorporated.

Wardman, M. and Bristow, A. L. 2004. Traffic related noise and air quality valuations: evidence from stated preference residential choice models. Transportation Research Part D: Transport and Environment 9(1), pp. 1-27.

Watkins, C. A. 2001. The definition and identification of housing submarkets. Environment and Planning A 33(12), pp. 2235-2254.

Webster, C. 2010. Pricing accessibility: Urban morphology, design and missing markets. Progress in Planning 73(2), pp. 77-111.

Weibull, J. W. 1976. An axiomatic approach to the measurement of accessibility. Regional science and urban economics 6(4), pp. 357-379.

White, M. J. 1988a. Location choice and commuting behavior in cities with decentralized employment. Journal of Urban Economics 24(2), pp. 129-152.

White, M. J. 1988b. Urban commuting journeys are not" wasteful". The journal of political economy 96(5), pp. 1097-1110.

Whitehand, J. 1972. Building cycles and the spatial pattern of urban growth. Transactions of the Institute of British Geographers, pp. 39-55.

Whitehand, J. 1987a. MRG Conzen and the intellectual parentage of urban morphology. Planning History Bulletin 9(2), pp. 35-41.

Whitehand, J. 1987b. Urban morphology. Historical geography: progress and prospect, pp. 250-276.

Whitehand, J. W. R. 1967. Fringe belts: a neglected aspect of urban geography. 
Transactions of the Institute of British Geographers, pp. 223-233.

Whitehand, J. W. R. 1992. Recent advances in urban morphology. Urban studies 29(3-4), pp. 619-636.

Whitehead, C. M. E. 1999. Urban housing markets: theory and policy. Handbook of regional and urban economics 3, pp. 1559-1594.

Whitehead, C. M. E. and Odling-Smee, J. 1975. Long-run equilibrium in urban housing-a note. Urban Studies 12(3), p. 315.

Wilman, E. A. 1981. Hedonic prices and beach recreational values. Advances in Applied Microeconomics 1(1), p. 98.

Wilson, A. G. 1970. Entropy in urban and regional modelling. Pion Ltd.

Wright, F. L. 1935. Broadacre City: A new community plan. Architectural Record Publishing Company.

Yinger, J. 1979. ESTIMATING THE RELATIONSHIP BETWEEN LOCATION AND THE PRICE OF HOUSING*. Journal of Regional Science 19(3), pp. 271-286.

Zhang, K. H. and Song, S. 2003. Rural-urban migration and urbanization in China: Evidence from time-series and cross-section analyses. China Economic Review 14(4), pp. 386-400.

Zhang, L. and Zhao, S. X. 2009. City branding and the Olympic effect: A case study of Beijing. Cities 26(5), pp. 245-254. 


\section{Appendices}




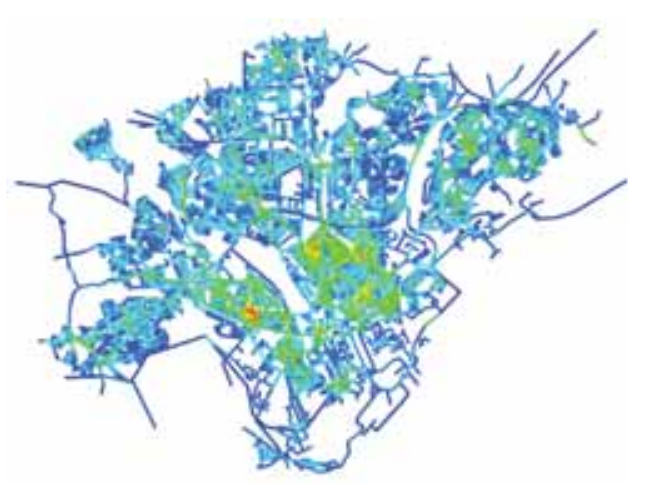

Figure A4. 1Integration at radii of $0.4 \mathrm{~km}$

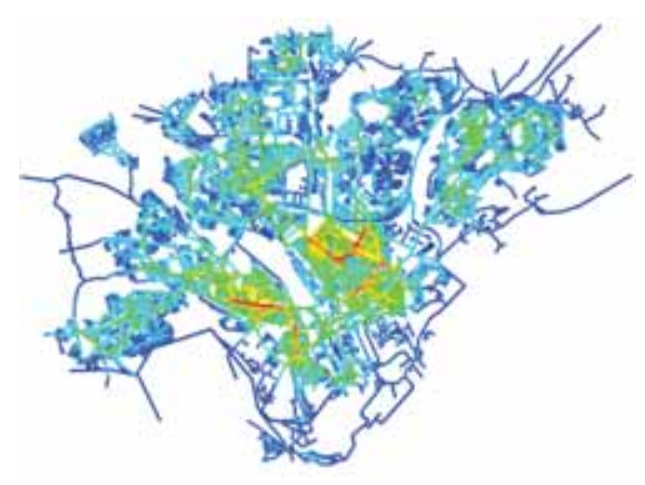

Figure A4. 2Integration at radii of $0.8 \mathrm{~km}$

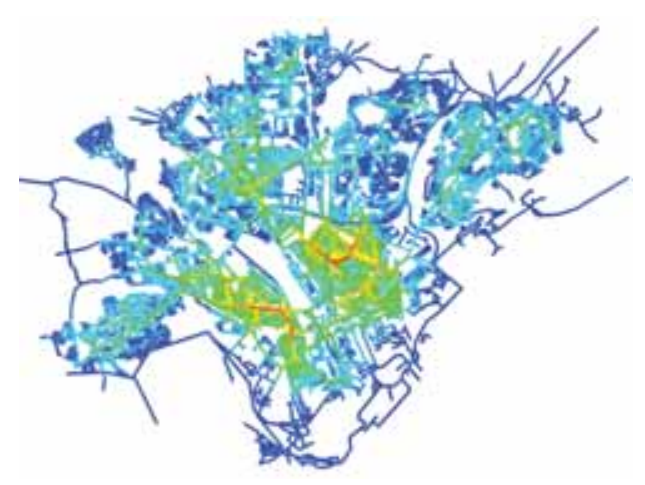

Figure A4. 3Integration at radii of $1.2 \mathrm{~km}$

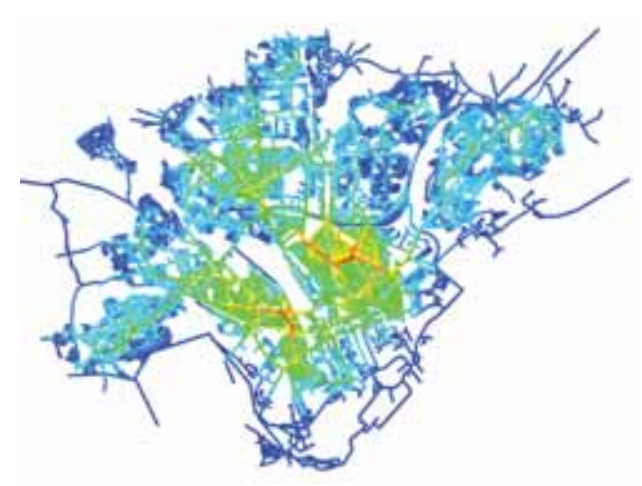

Figure A4. 4 Integration at radii of $1.6 \mathrm{~km}$

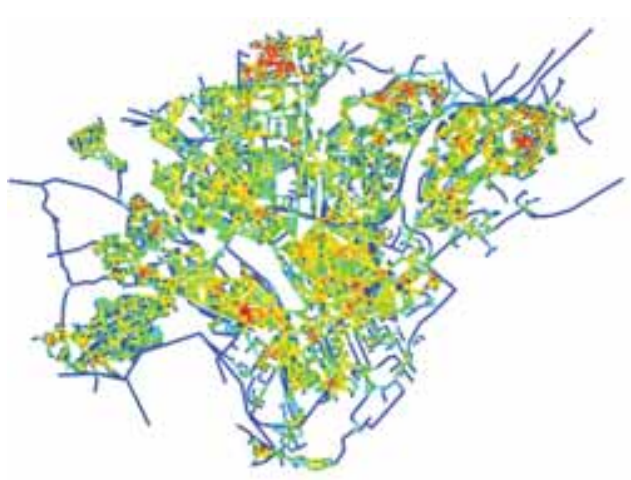

Figure A4. 5 Choice at radii of $0.4 \mathrm{~km}$

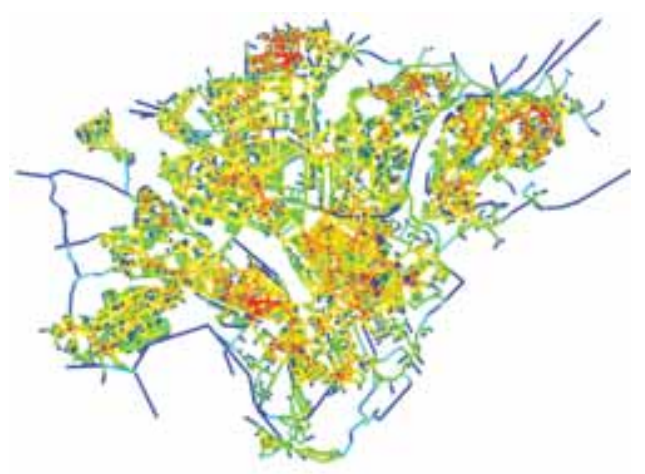

Figure A4. 6 Choice at radii of $0.8 \mathrm{~km}$

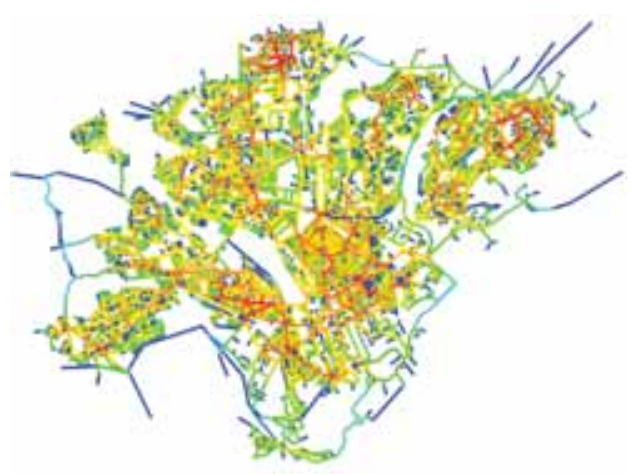

Figure A4. 7 Choice at radii of $1.2 \mathrm{~km}$

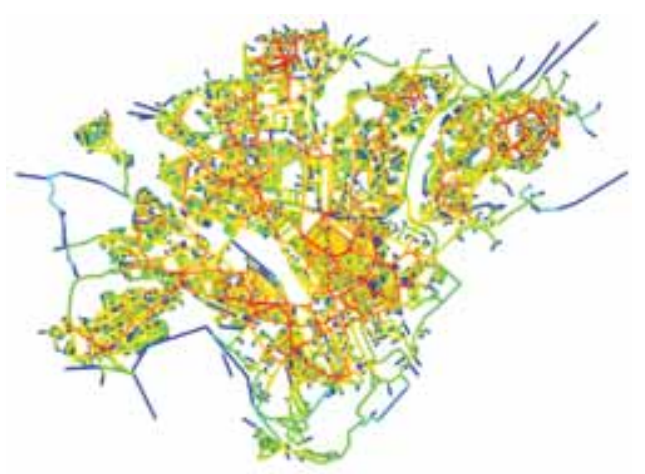

Figure A4. 8 Choice at radii of $1.6 \mathrm{~km}$ 


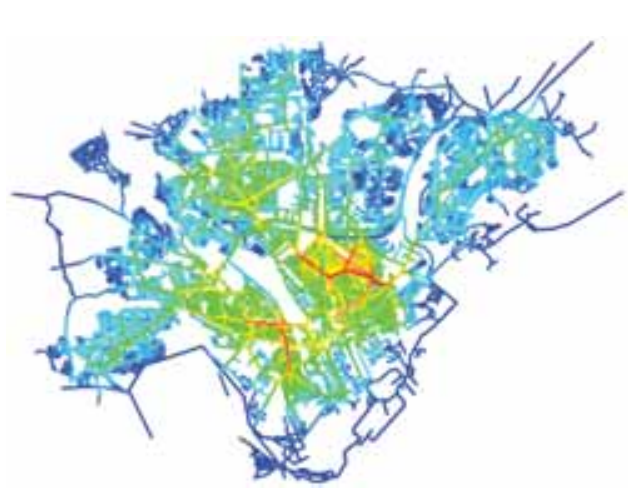

Figure A4. 9 Integration at radii of $2 \mathrm{~km}$

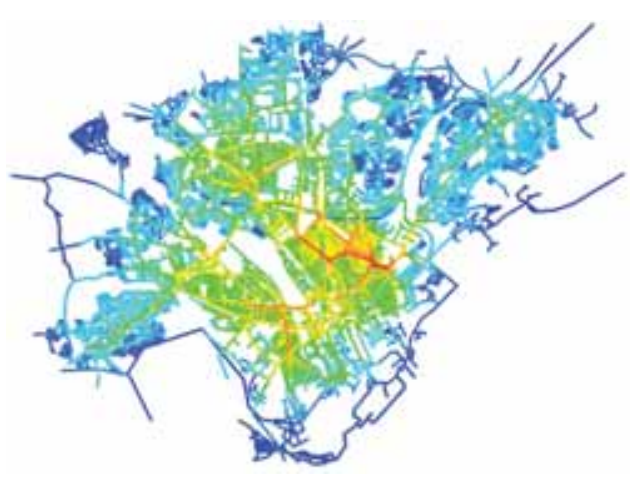

Figure A4. 10 Integration at radii of $2.5 \mathrm{~km}$

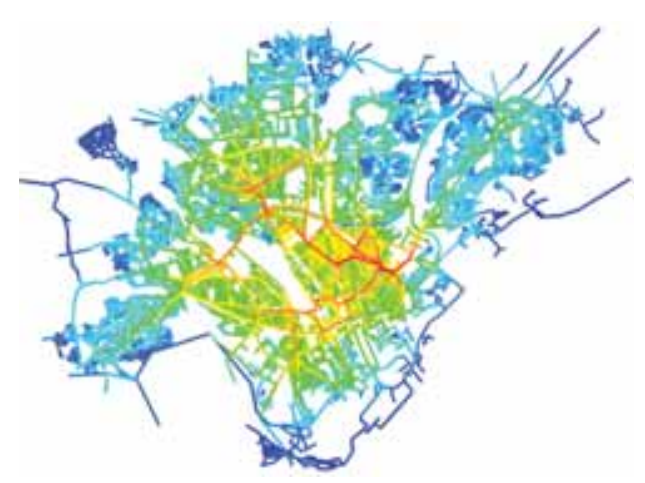

Figure A4. 11 Integration at radii of $3 \mathrm{~km}$

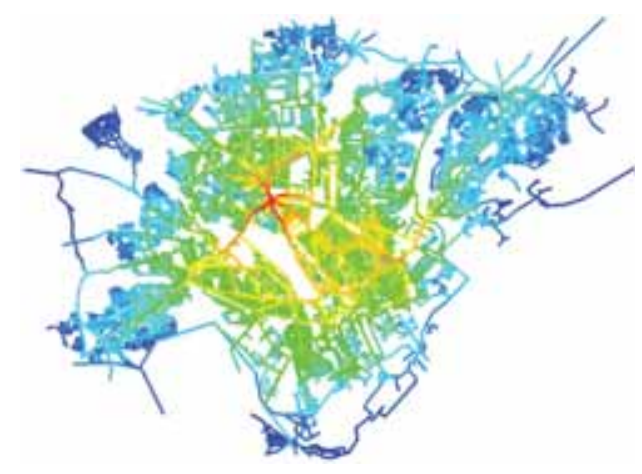

Figure A4. 12 Integration at radii of $4 \mathrm{~km}$

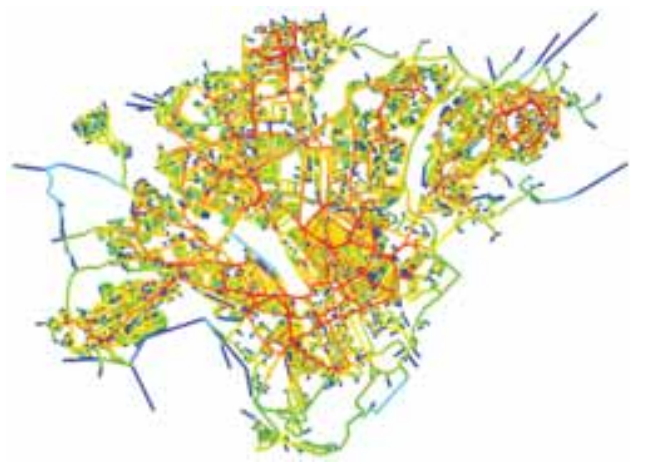

Figure A4. 13 Choice at radii of $2 \mathrm{~km}$

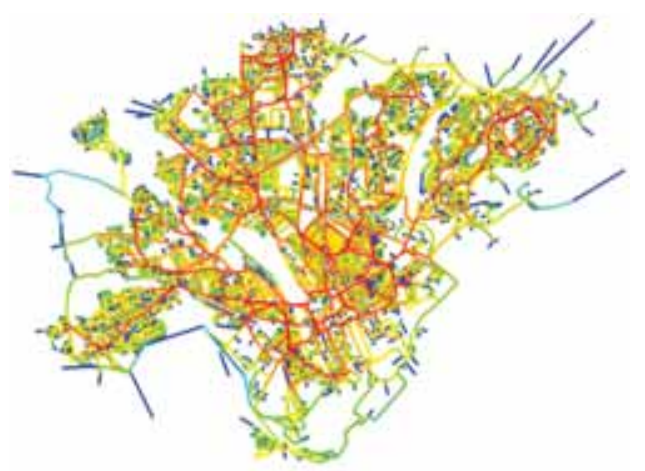

Figure A4. 14 Choice at radii of $2.5 \mathrm{~km}$

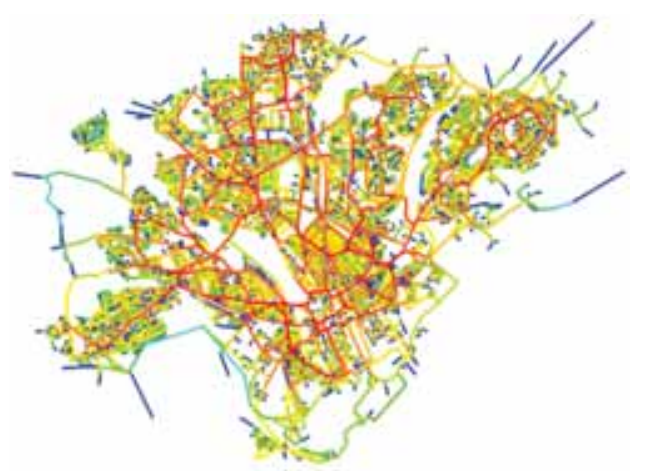

Figure A4. 15 Choice at radii of $3 \mathrm{~km}$

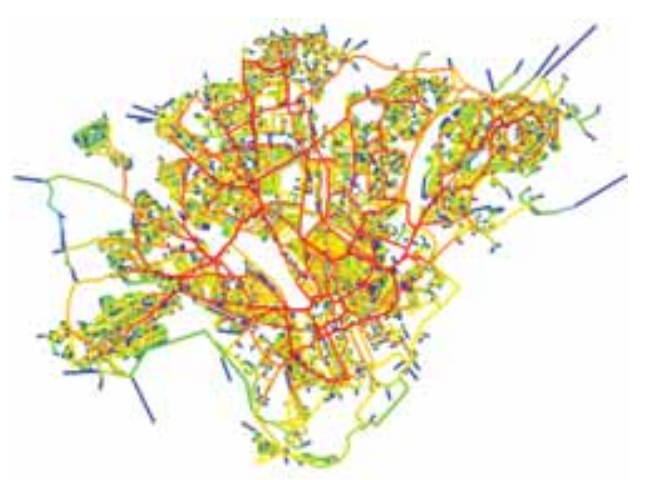

Figure A4. 16 Choice at radii of $4 \mathrm{~km}$ 


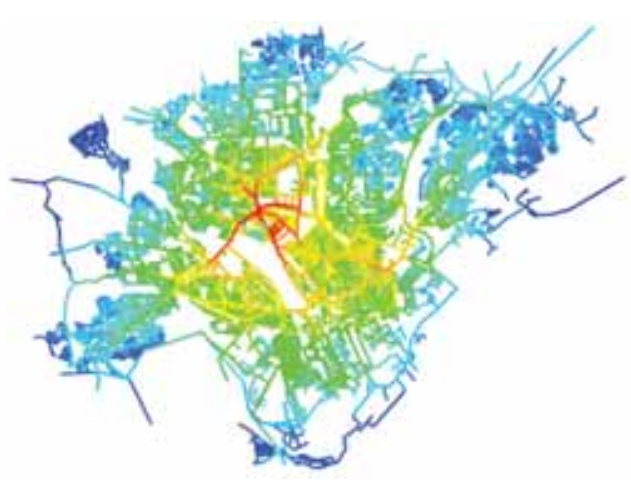

Figure A4. 17 Integration at radii of $5 \mathrm{~km}$

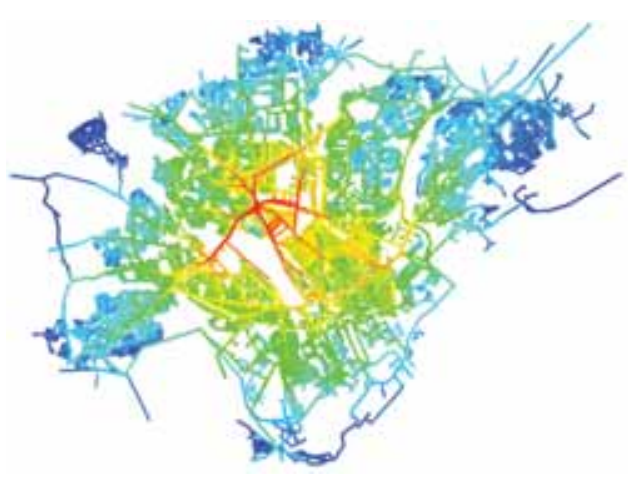

Figure A4. 18 Integration at radii of $6 \mathrm{~km}$

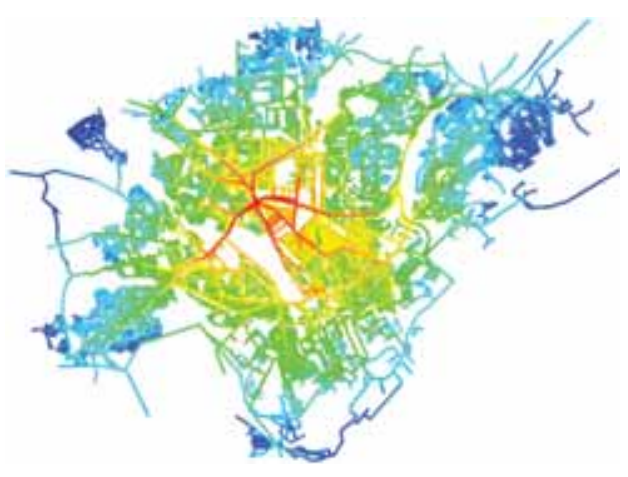

Figure A4. 19 Integration at radii of $7 \mathrm{~km}$

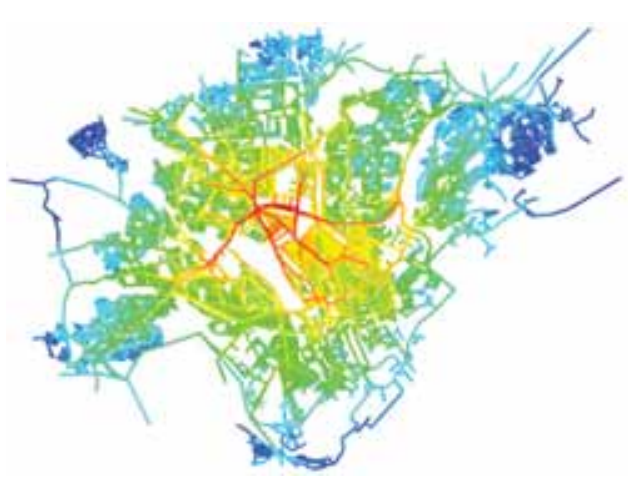

Figure A4. 20 Integration at radii of $8 \mathrm{~km}$

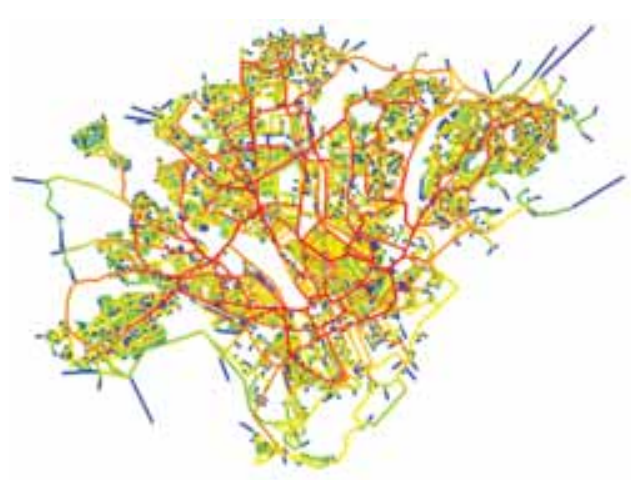

Figure A4. 21 Choice at radii of $5 \mathrm{~km}$

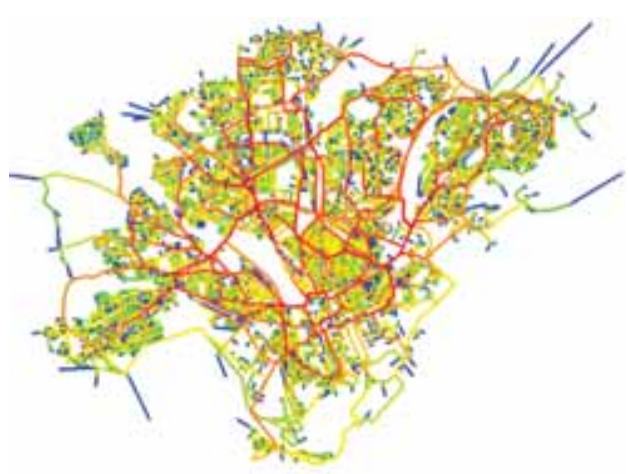

Figure A4. 22 Choice at radii of $6 \mathrm{~km}$

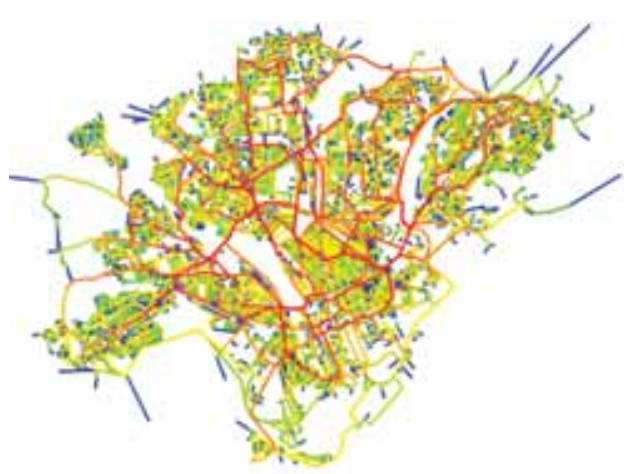

Figure A4. 23 Choice at radii of $7 \mathrm{~km}$

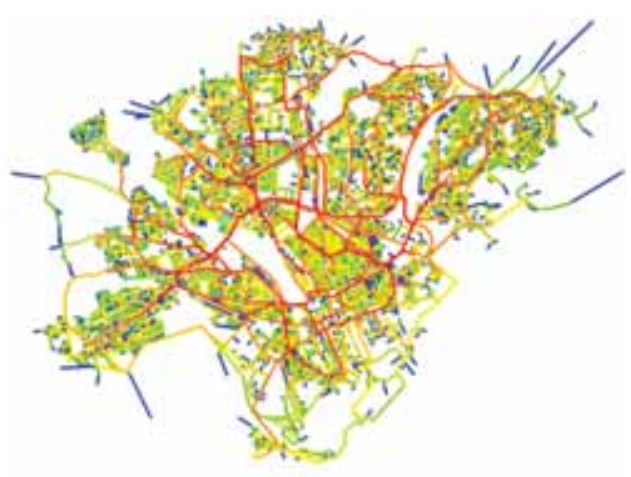

Figure A4. 24 Choice at radii of $8 \mathrm{~km}$ 


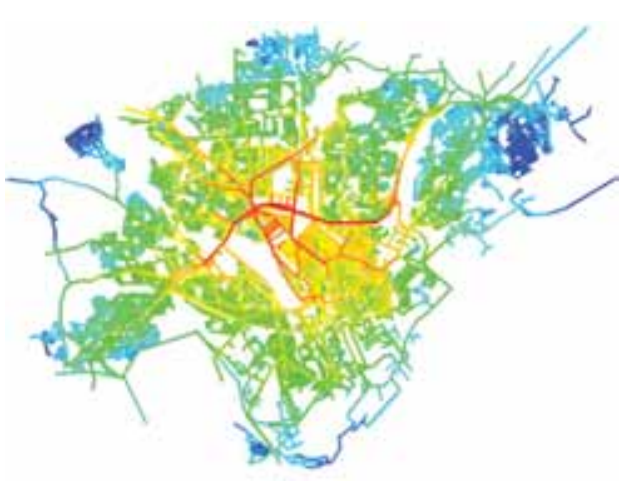

Figure A4. 25Integration at radii of $10 \mathrm{~km}$

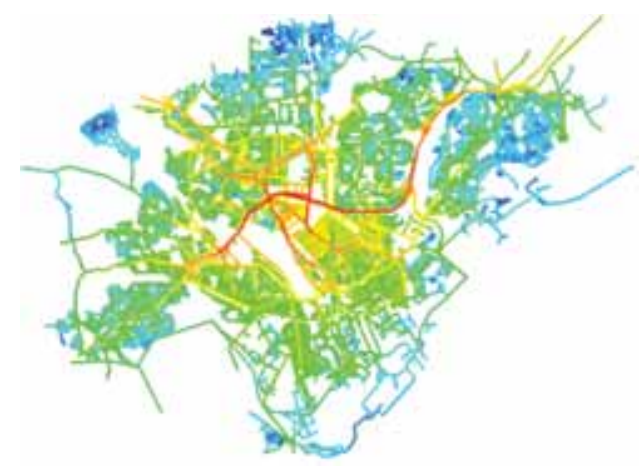

Figure A4. 26 Global integration

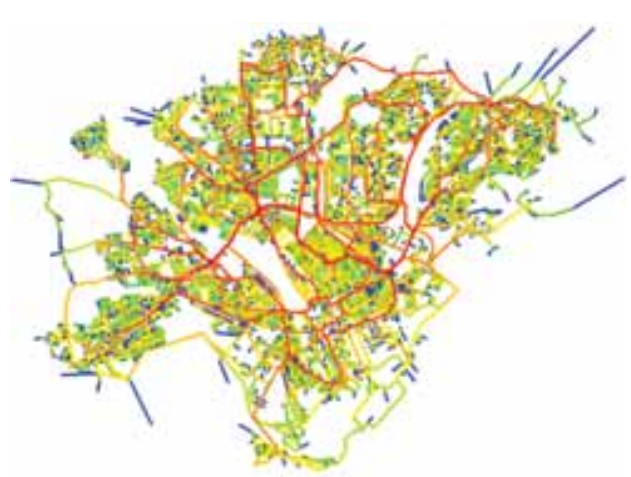

Figure A4. 27 Choice at radii of $10 \mathrm{~km}$

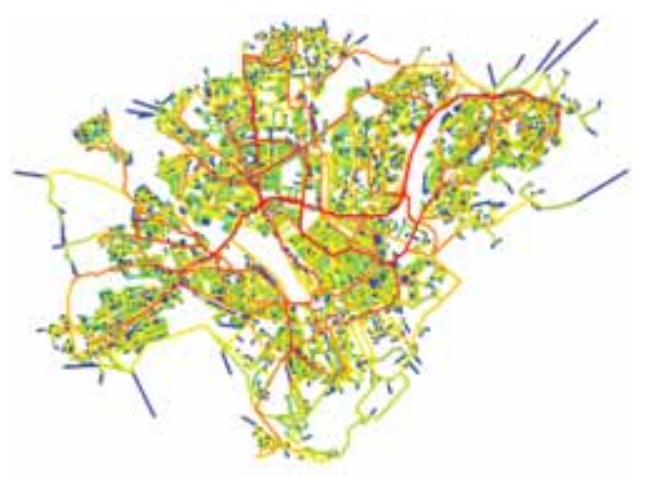

Figure A4. 28 Global choice 


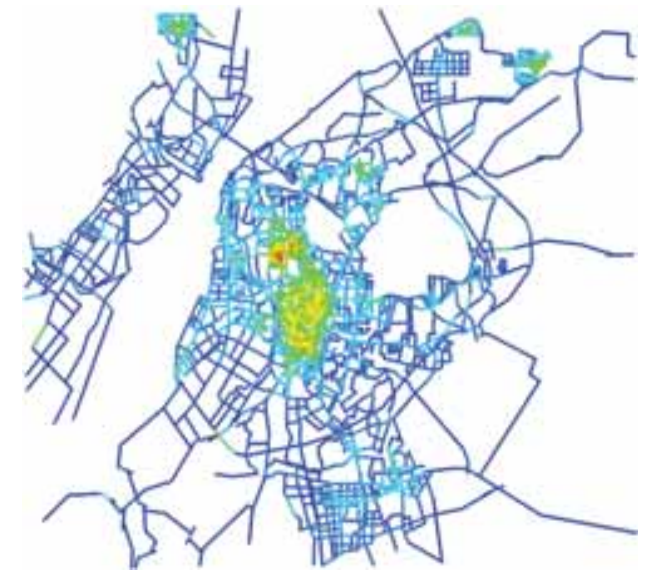

Figure A6. 1 Integration at $0.8 \mathrm{~km}$ in 2005

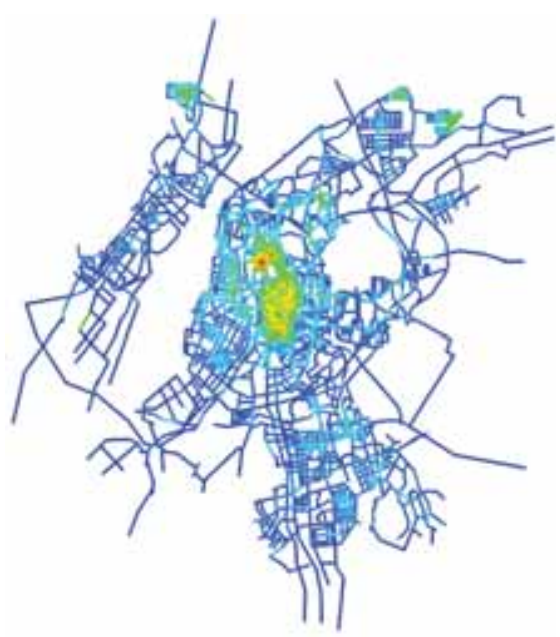

Figure A6. 2Integration at $0.8 \mathrm{~km}$ in 2006

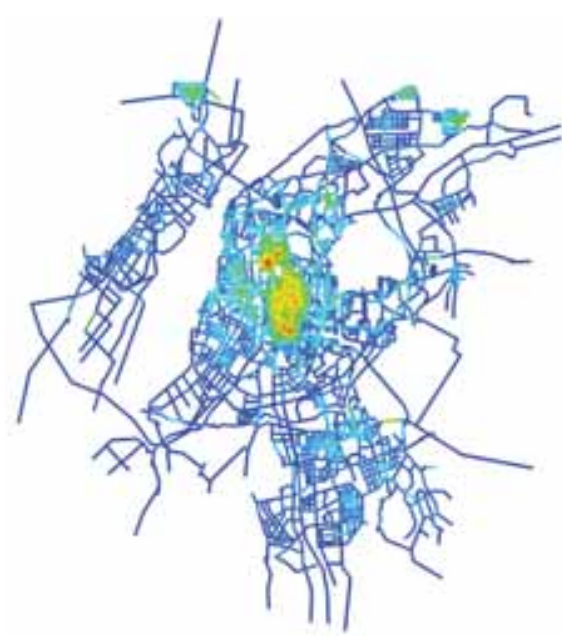

Figure A6. 3Integration at $0.8 \mathrm{~km}$ in 2007

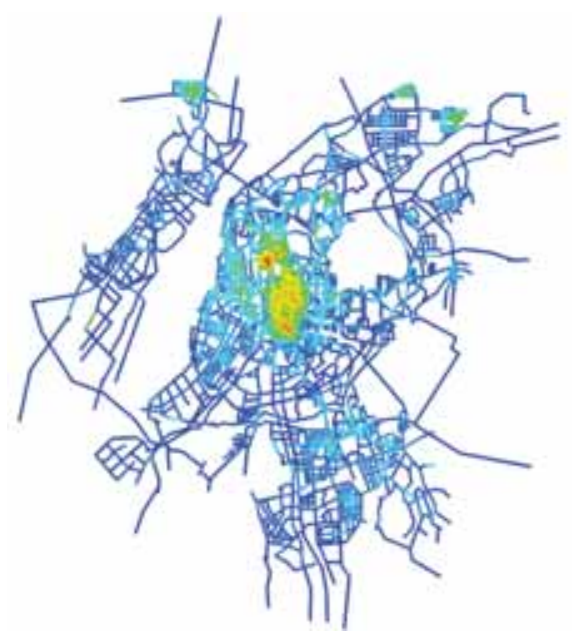

Figure A6. 4Integration at $0.8 \mathrm{~km}$ in 2008

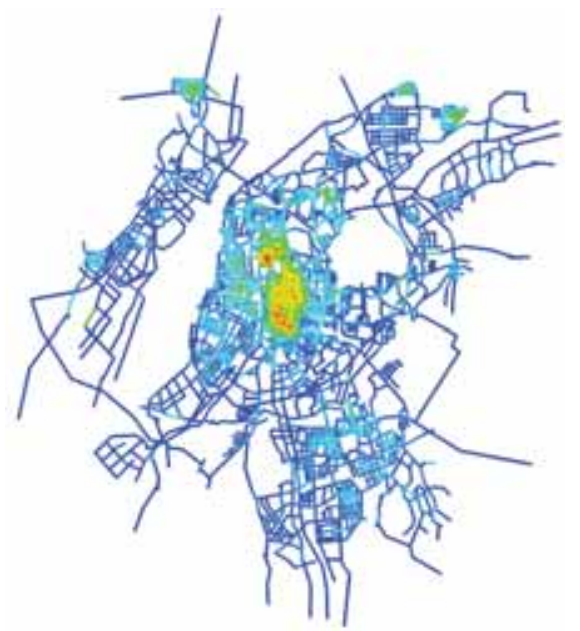

Figure A6. 5Integration at $0.8 \mathrm{~km}$ in 2009

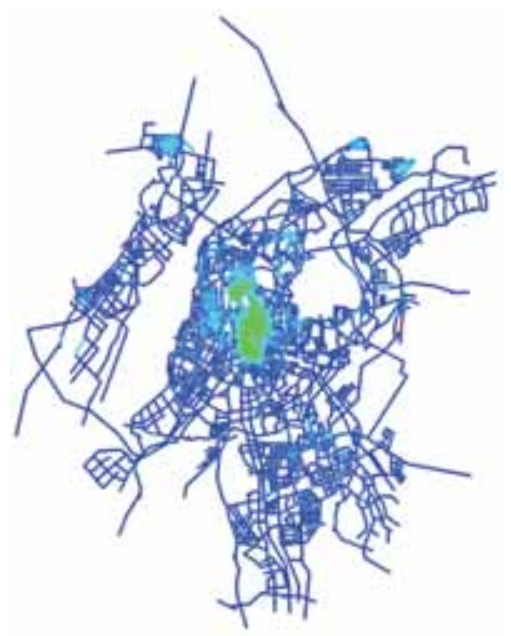

Figure A6. 6Integration at $0.8 \mathrm{~km}$ in 2010 


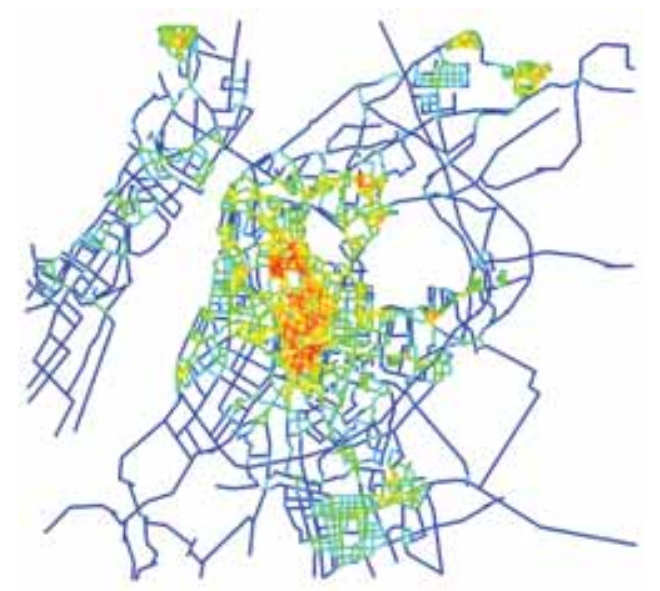

Figure A6. 7 Choice at $0.8 \mathrm{~km}$ in 2005

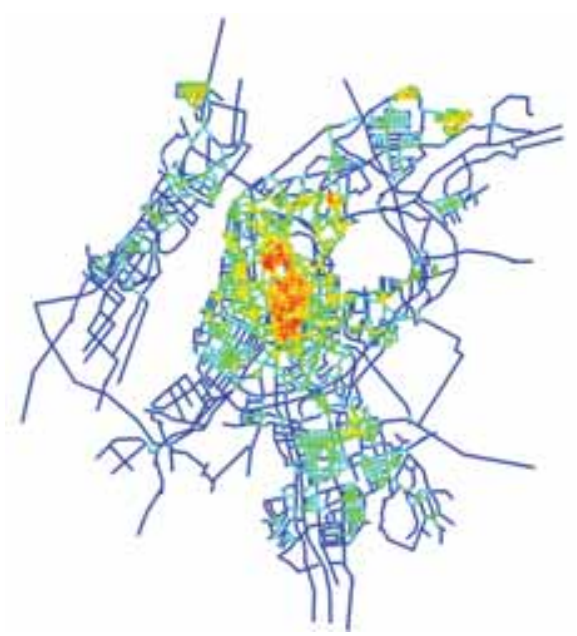

Figure A6. 8Choice at $0.8 \mathrm{~km}$ in 2006

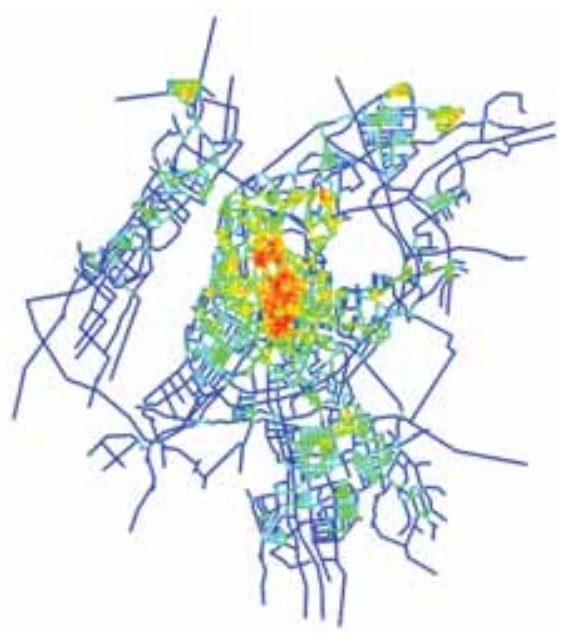

Figure A6. 9Choice at $0.8 \mathrm{~km}$ in 2007

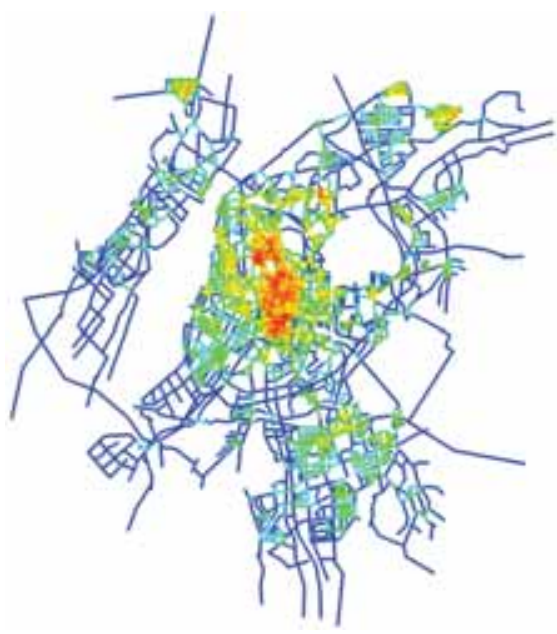

Figure A6. 10Choice at $0.8 \mathrm{~km}$ in 2008

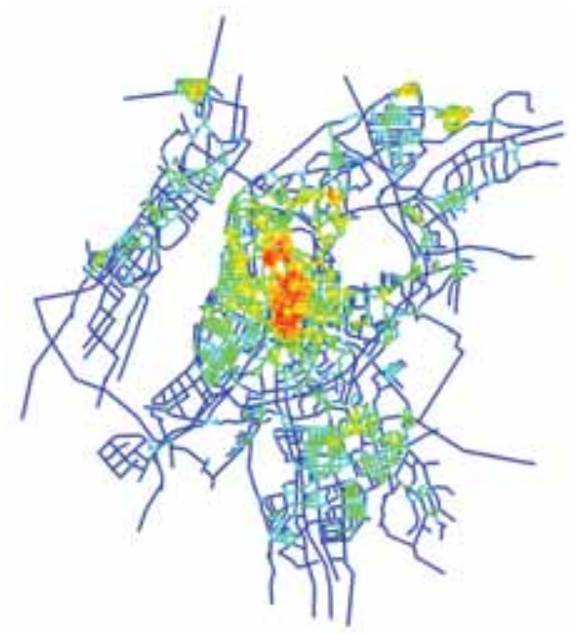

Figure A6. 11Choice at $0.8 \mathrm{~km}$ in 2009

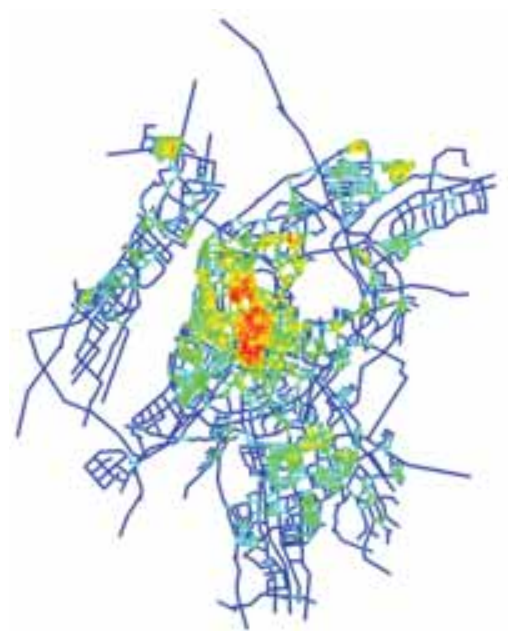

Figure A6. 12Choice at $0.8 \mathrm{~km}$ in 2010 


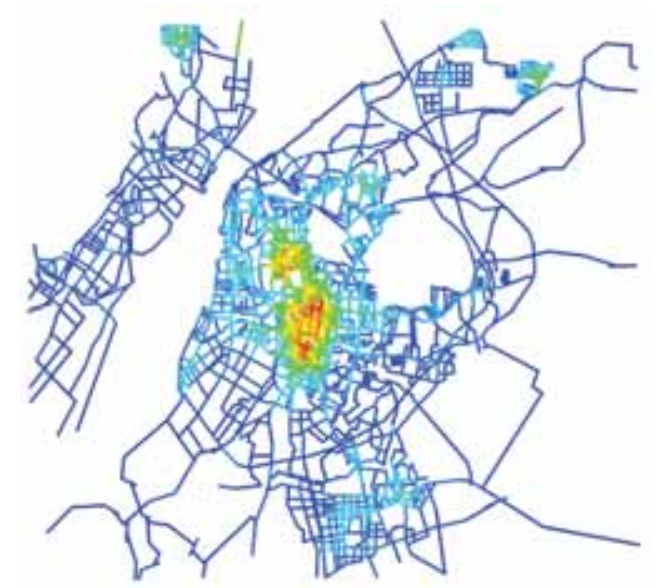

Figure A6. 13Integrationat $1.2 \mathrm{~km}$ in 2005

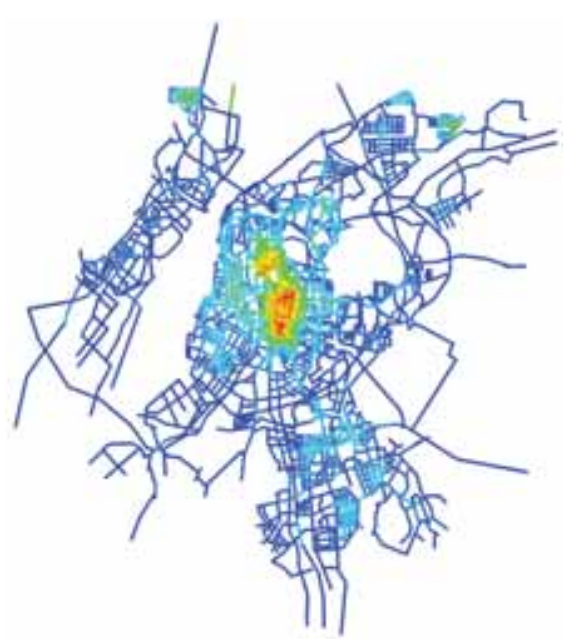

Figure A6. 14Integrationat $1.2 \mathrm{~km}$ in 2006

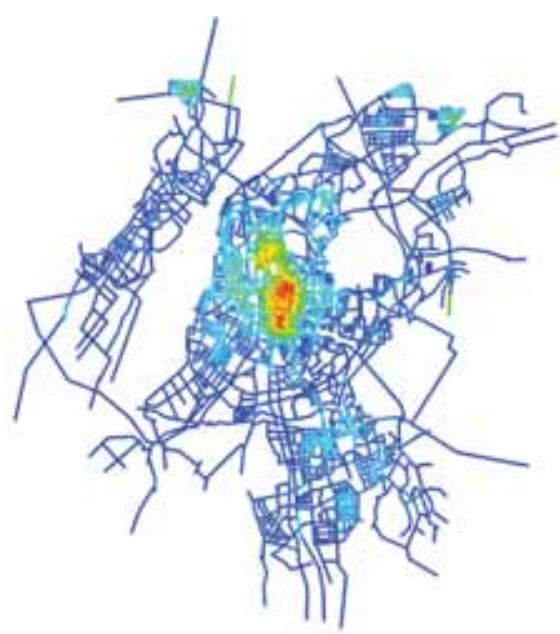

Figure A6. 15Integration at $1.2 \mathrm{~km}$ in 2007

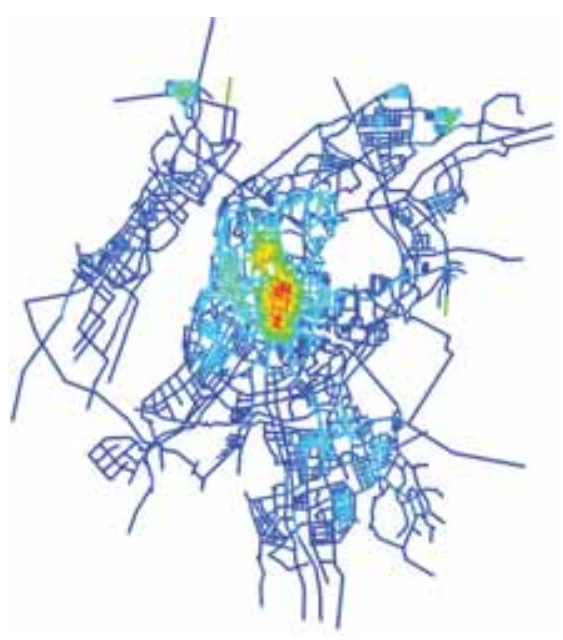

Figure A6. 16Integration at $1.2 \mathrm{~km}$ in 2008

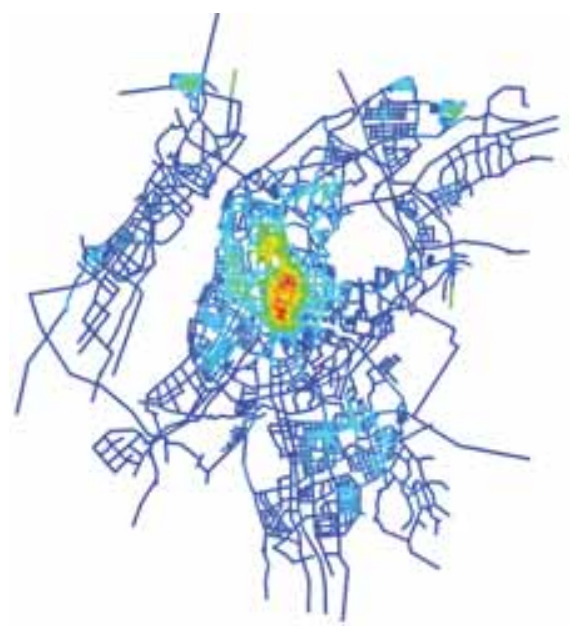

Figure A6. 17Integration at $1.2 \mathrm{~km}$ in 2009

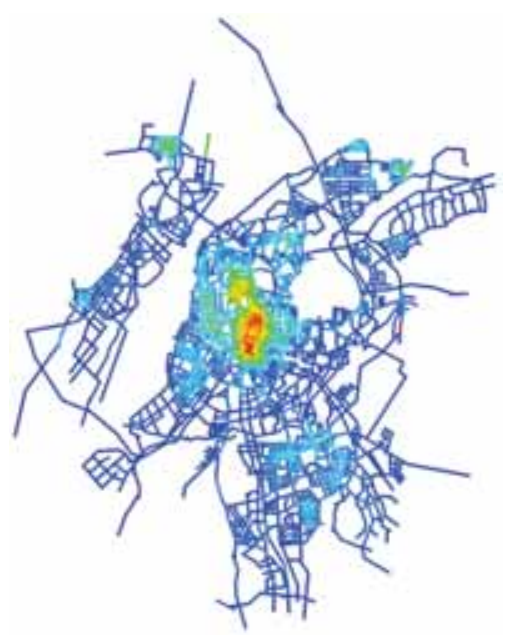

Figure A6. 18Integration at $1.2 \mathrm{~km}$ in 2010 


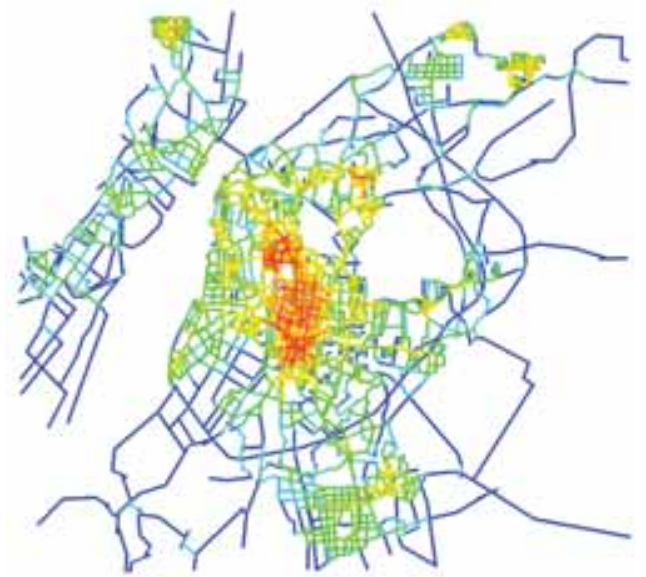

Figure A6. 19 Choice at $1.2 \mathrm{~km}$ in 2005

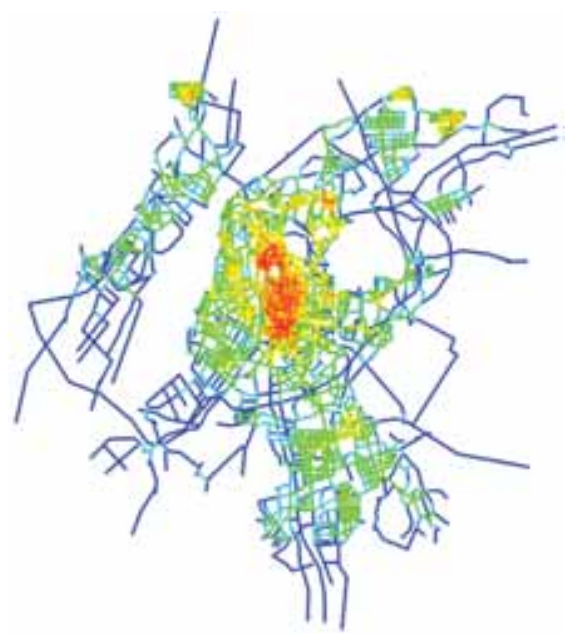

Figure A6. 20Choice at $1.2 \mathrm{~km}$ in 2006

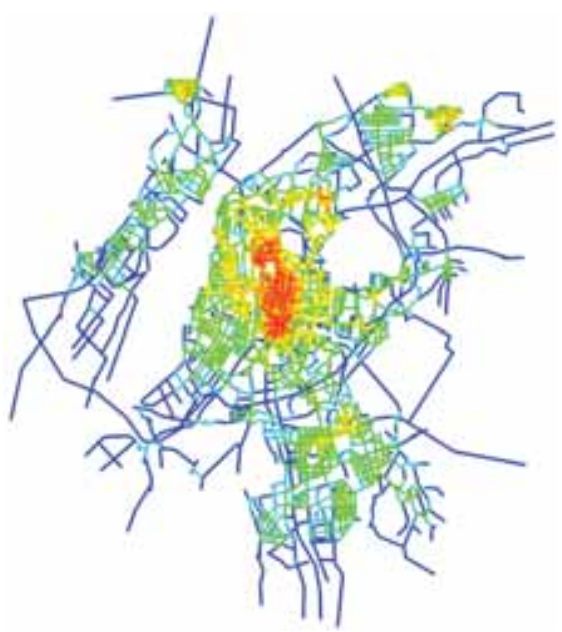

Figure A6. 21 Choice at $1.2 \mathrm{~km}$ in 2007

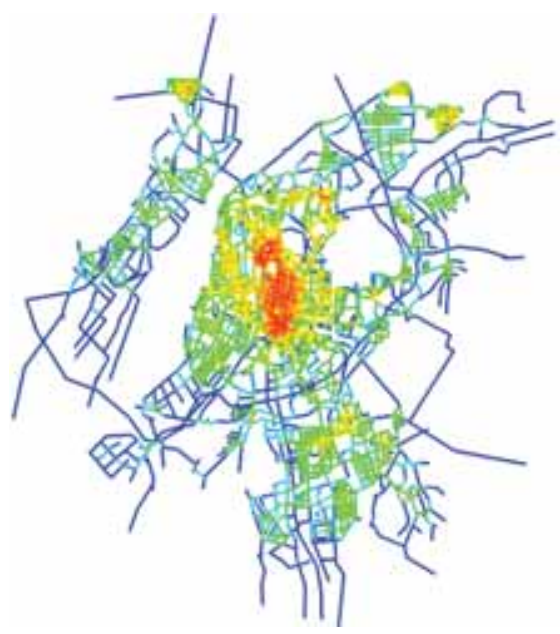

Figure A6. 22Choice at $1.2 \mathrm{~km}$ in 2008

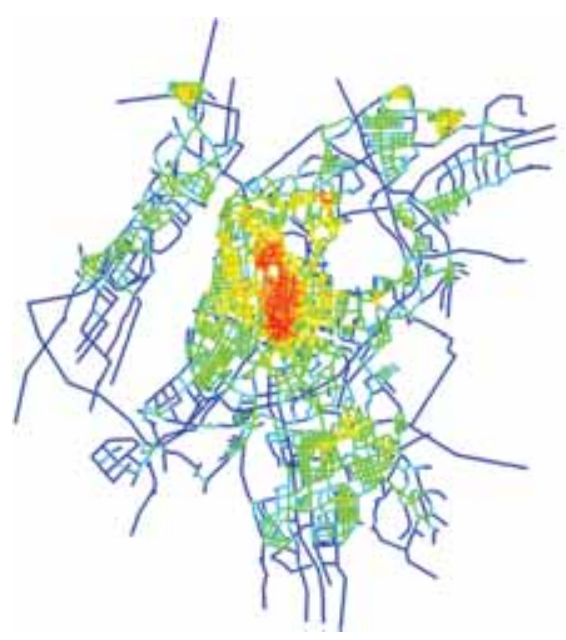

Figure A6. 23 Choice at $1.2 \mathrm{~km}$ in 2009

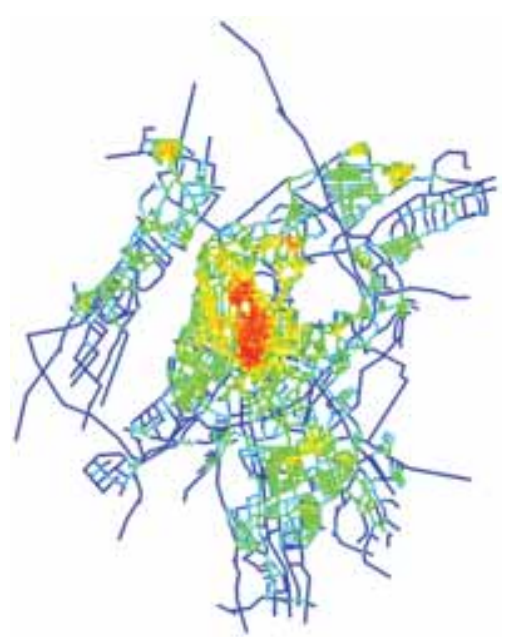

Figure A6. 24 Choice at $1.2 \mathrm{~km}$ in 2010 


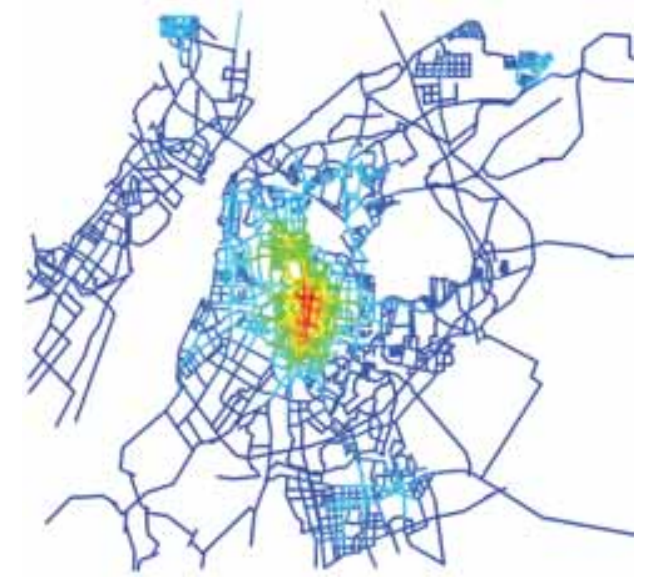

Figure A6. 25Integration at $2 \mathrm{~km}$ in 2005

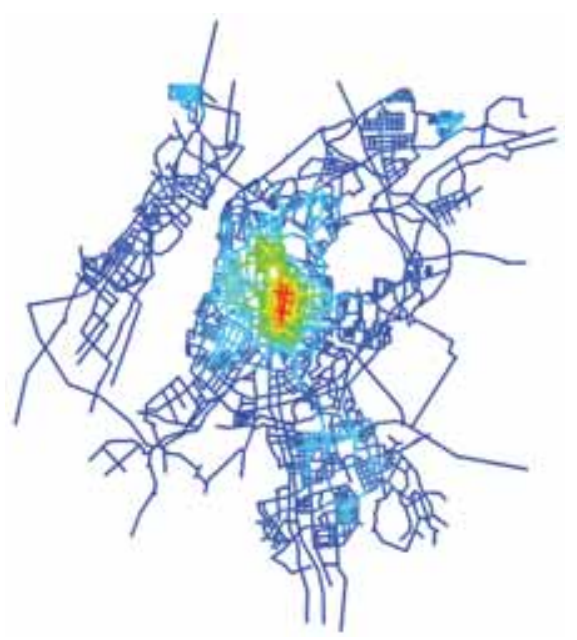

Figure A6. 26Integration at $2 \mathrm{~km}$ in 2006

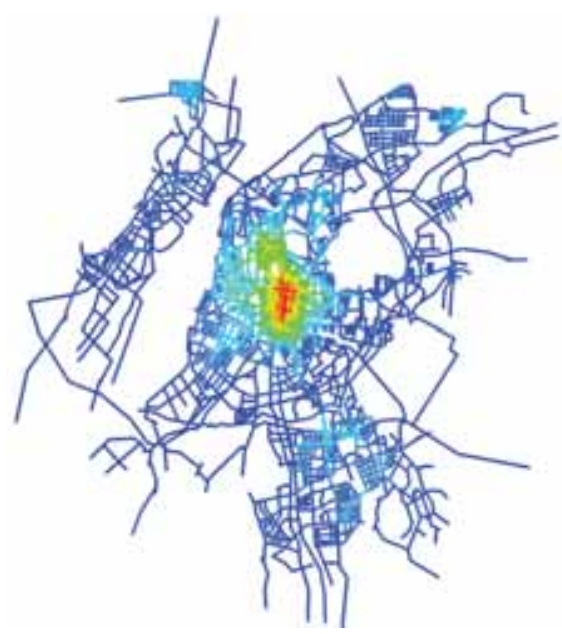

Figure A6. 27Integration at $2 \mathrm{~km}$ in 2007

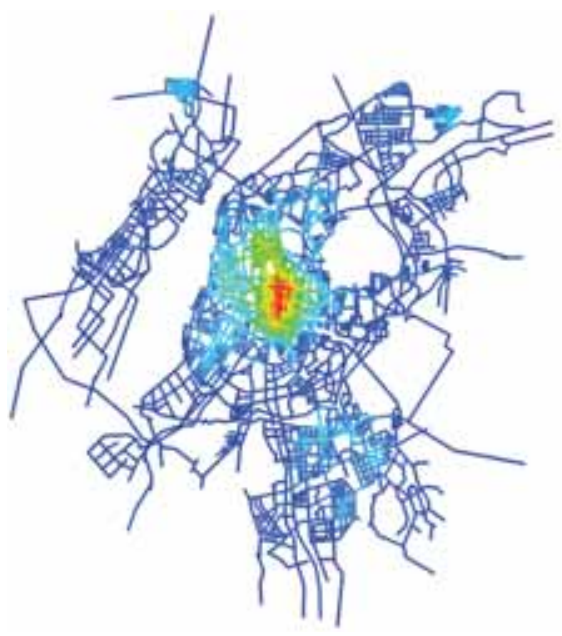

Figure A6. 28Integration at $2 \mathrm{~km}$ in 2008

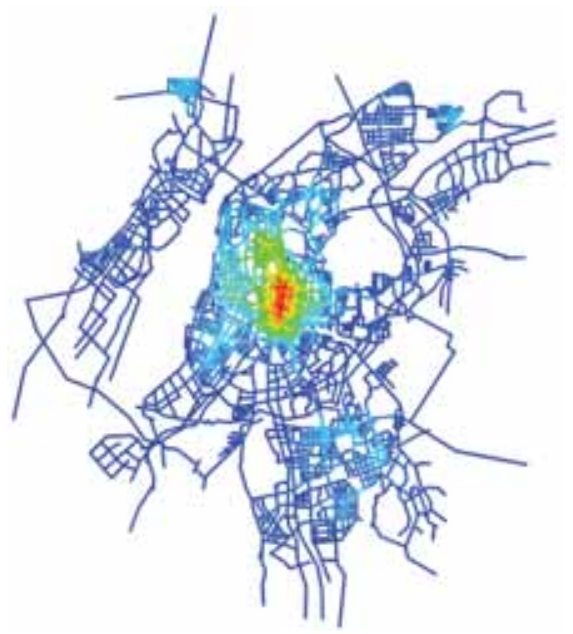

Figure A6. 29Integration at $2 \mathrm{~km}$ in 2009

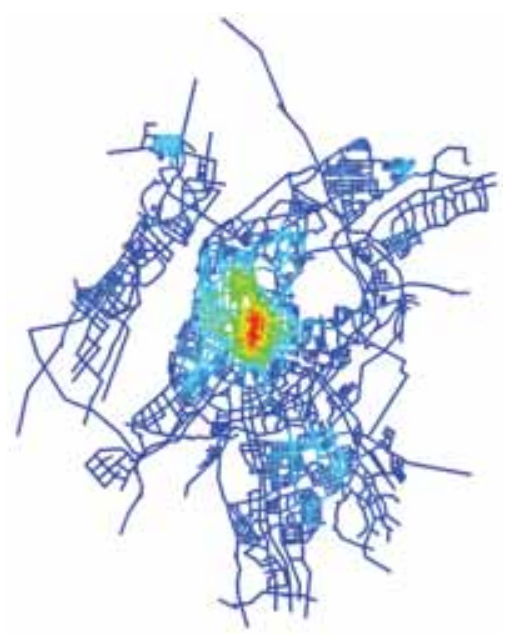

Figure A6. 30Integration at $2 \mathrm{~km}$ in 2010 


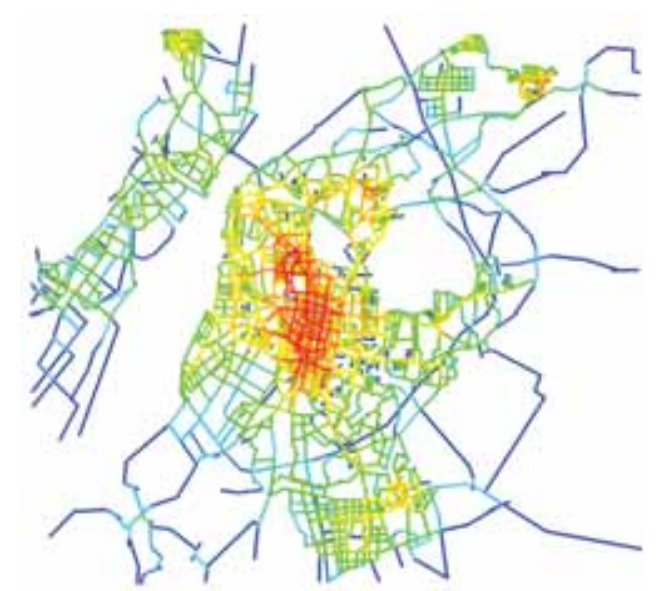

Figure A6. 31 Choice at $2 \mathrm{~km}$ in 2005

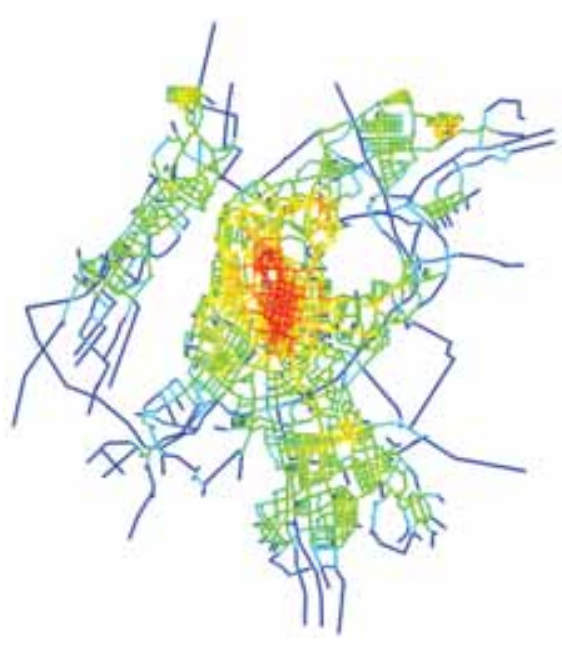

Figure A6. 32Choice at $2 \mathrm{~km}$ in 2006

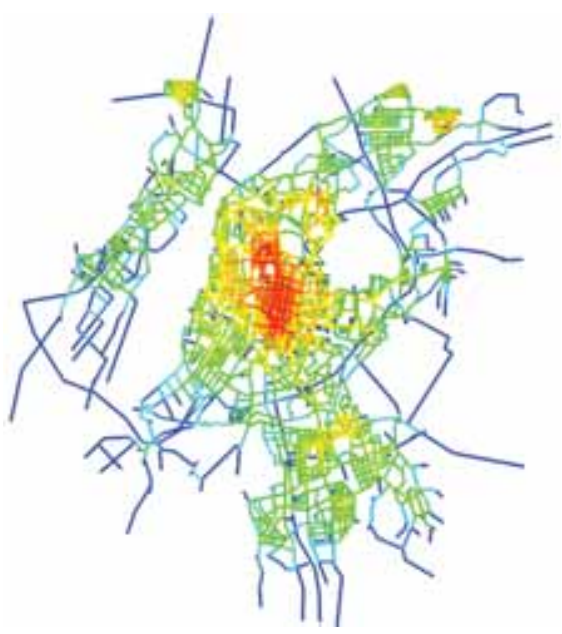

Figure A6. 33 Choice at $2 \mathrm{~km}$ in 2007

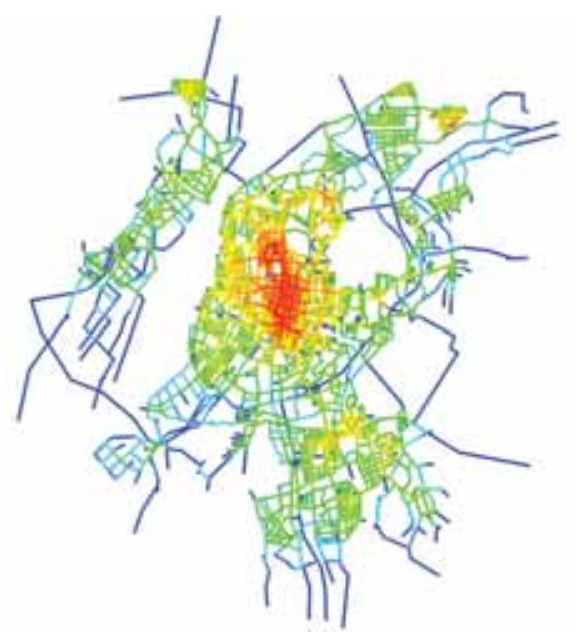

Figure A6. 34Choice at $2 \mathrm{~km}$ in 2008

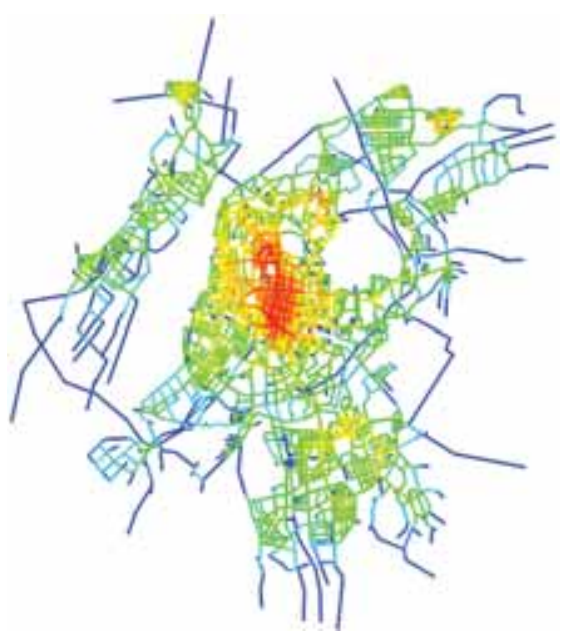

Figure A6. 35 Choice at $2 \mathrm{~km}$ in 2009

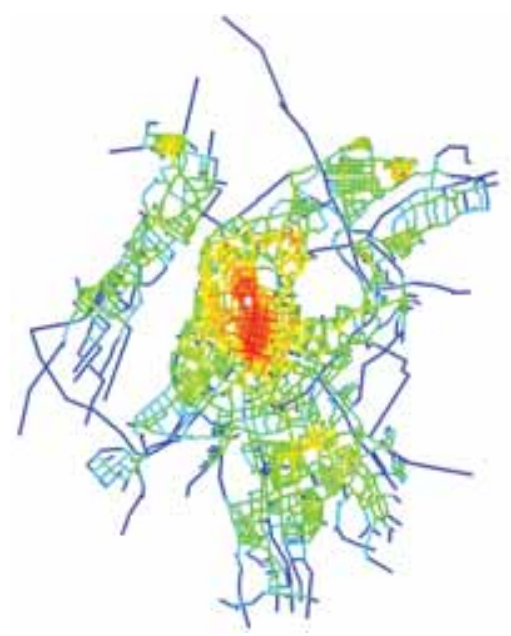

Figure A6. 36 Choice at $2 \mathrm{~km}$ in 2010 


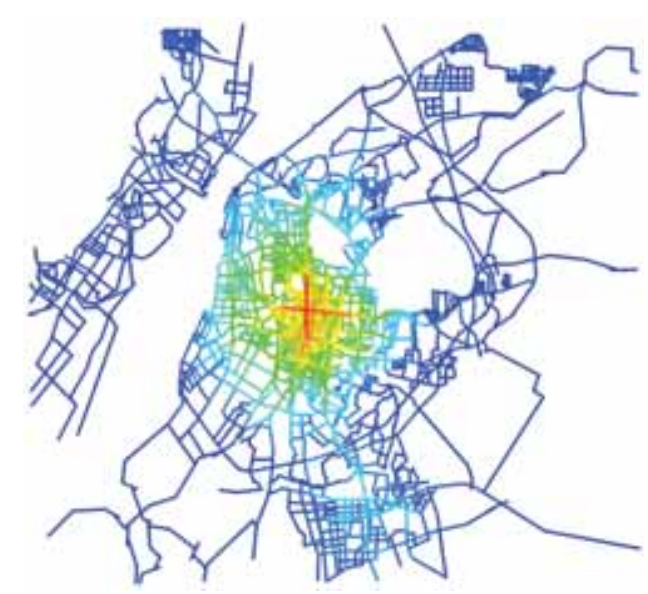

Figure A6. 37Integration at $5 \mathrm{~km}$ in 2005

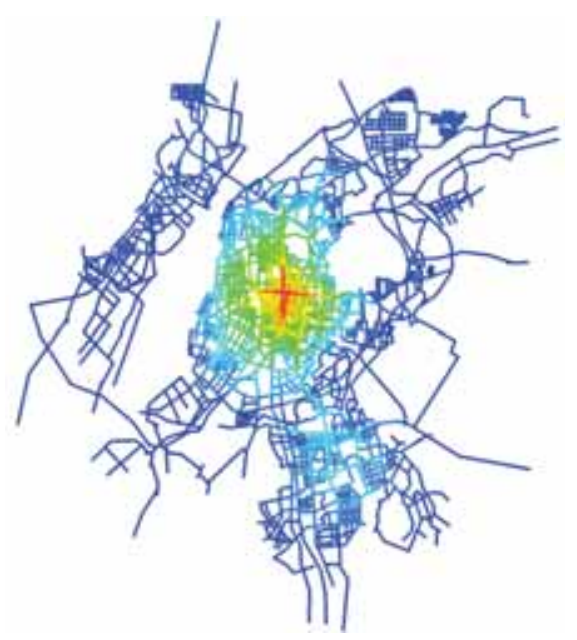

Figure A6. 38Integration at $5 \mathrm{~km}$ in 2006

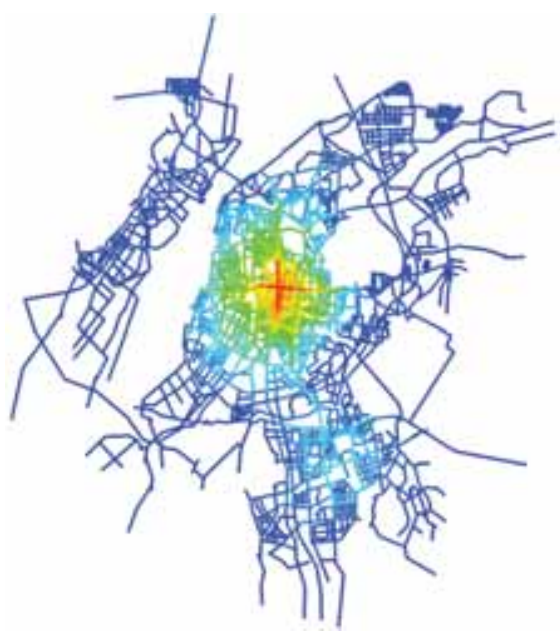

Figure A6. 39Integration at $5 \mathrm{~km}$ in 2007

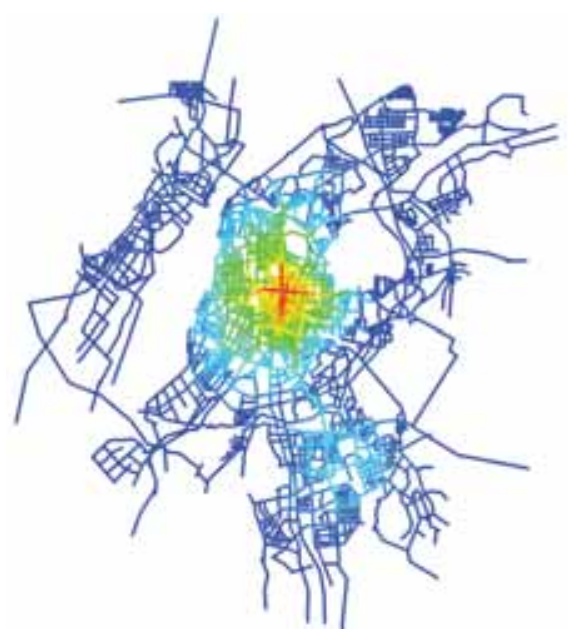

Figure A6. 40Integration at $5 \mathrm{~km}$ in 2008

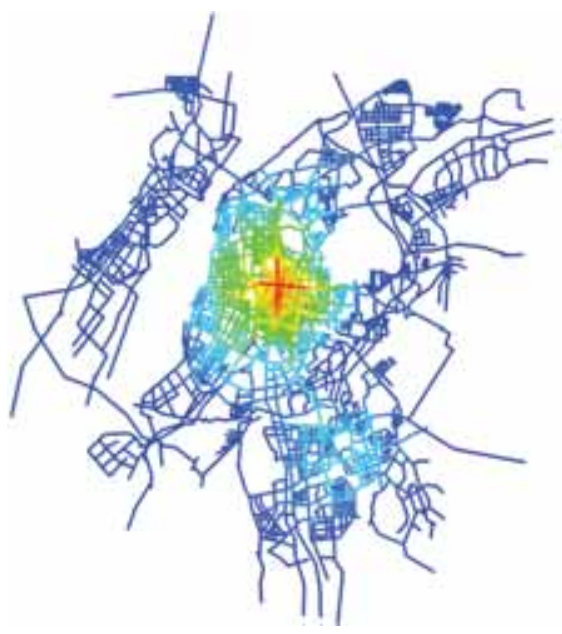

Figure A6. 41Integration at $5 \mathrm{~km}$ in 2009

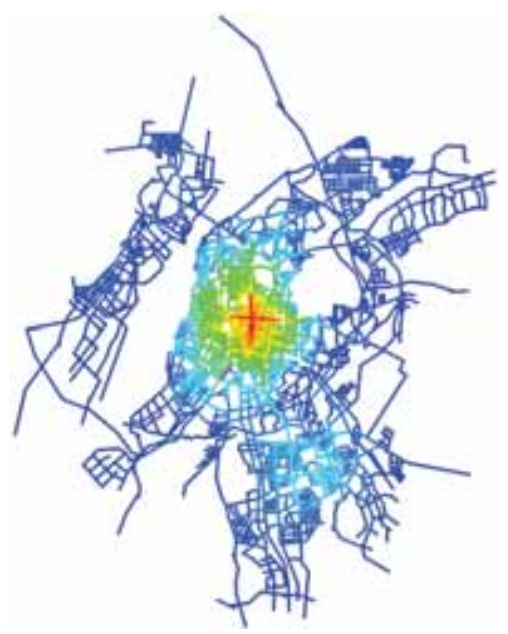

Figure A6. 42Integration at $5 \mathrm{~km}$ in 2010 


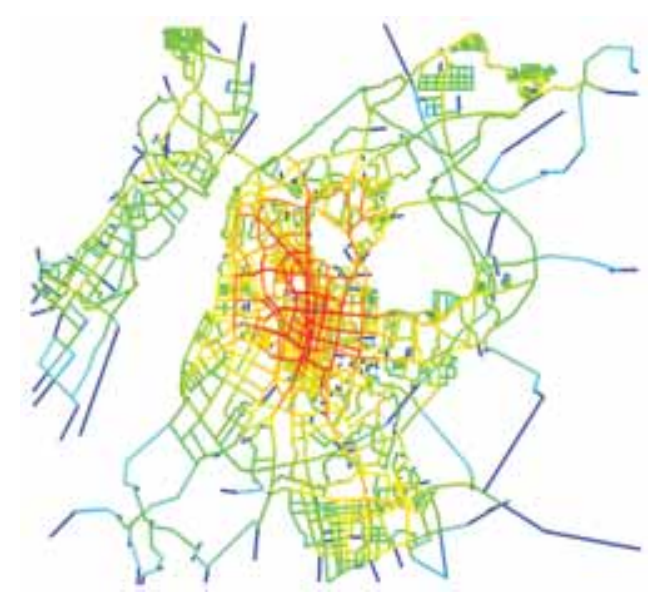

Figure A6. 43Choice at $5 \mathrm{~km}$ in 2005

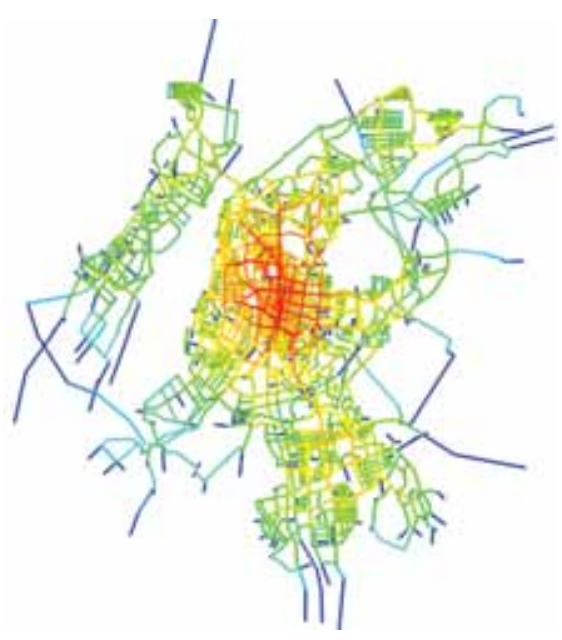

Figure A6. 44Choice at $5 \mathrm{~km}$ in 2006

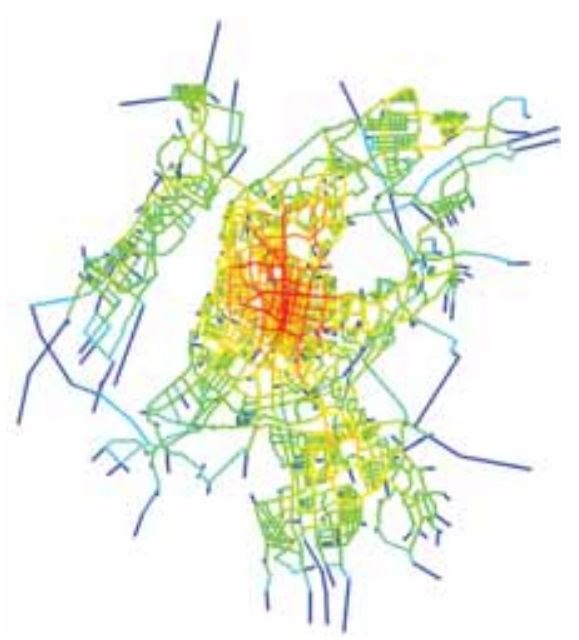

Figure A6. 45Choice at $5 \mathrm{~km}$ in 2007

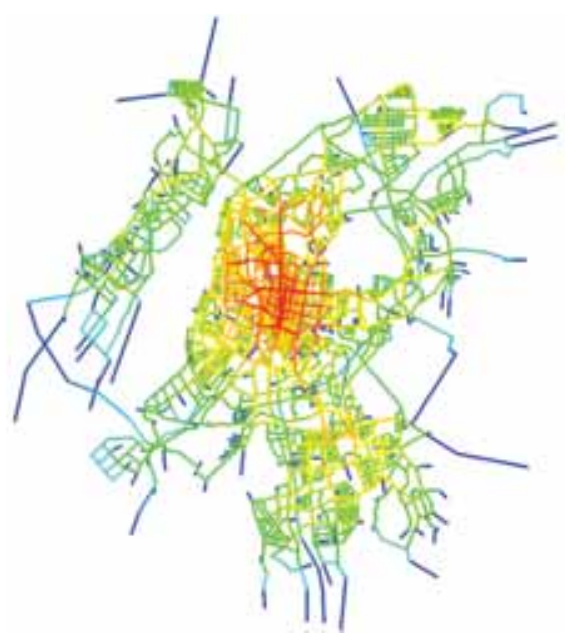

Figure A6. 46Choice at $5 \mathrm{~km}$ in 2008

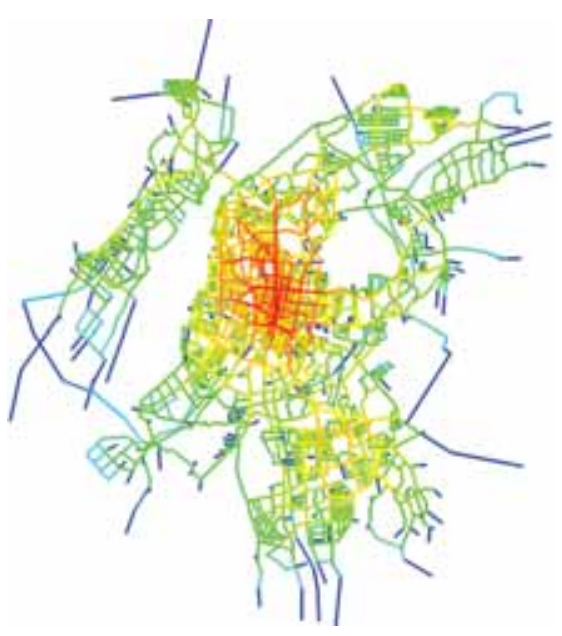

Figure A6. 47Choice at $5 \mathrm{~km}$ in 2009

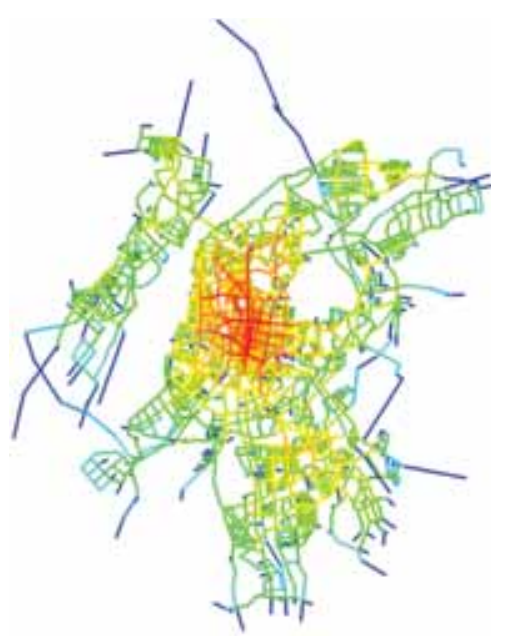

Figure A6. 48Choice at $5 \mathrm{~km}$ in 2010 


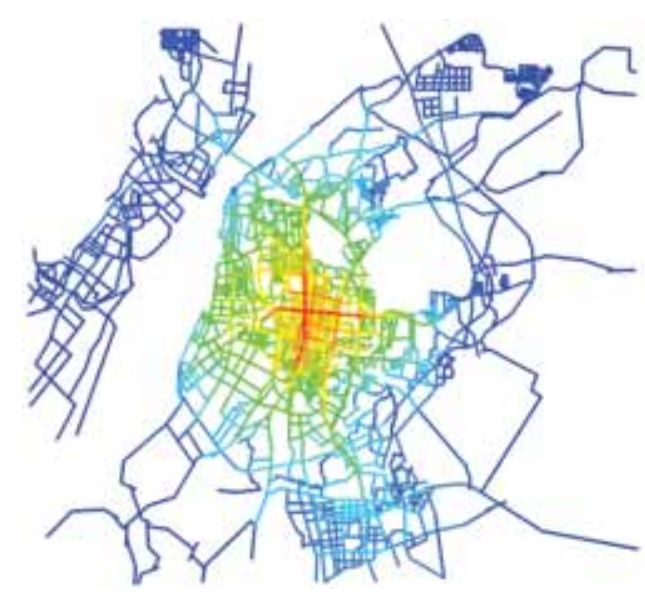

Figure A6. 49Integration at $8 \mathrm{~km}$ in 2005

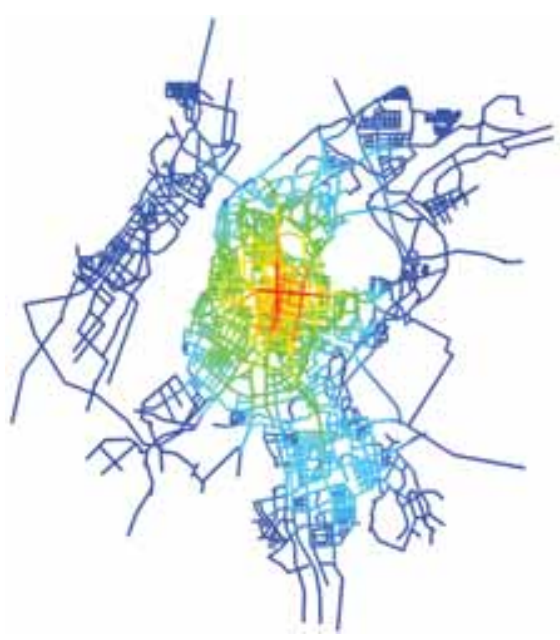

Figure A6. 50Integration at $6 \mathrm{~km}$ in 2006

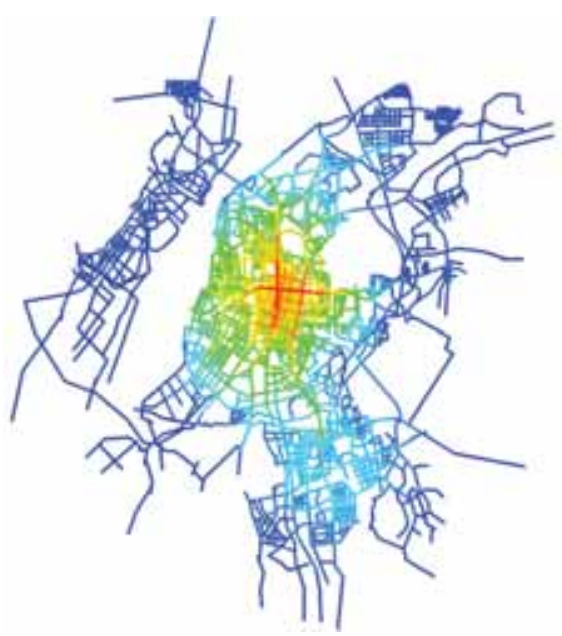

Figure A6. 51Integration at $8 \mathrm{~km}$ in 2007

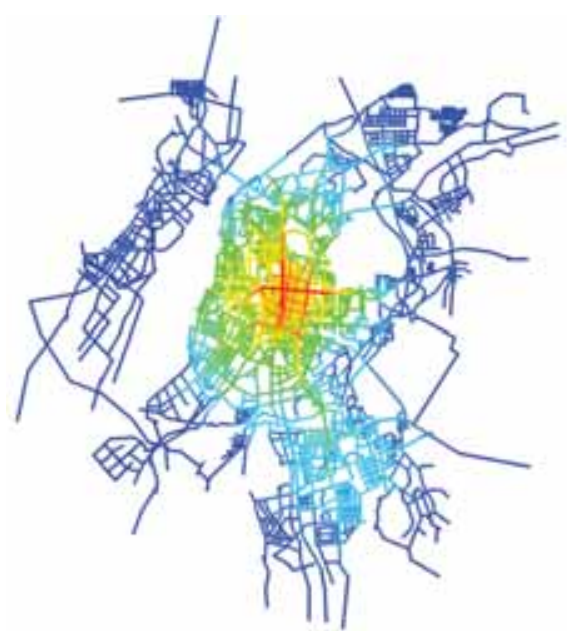

Figure A6. 52Integration at $8 \mathrm{~km}$ in 2008

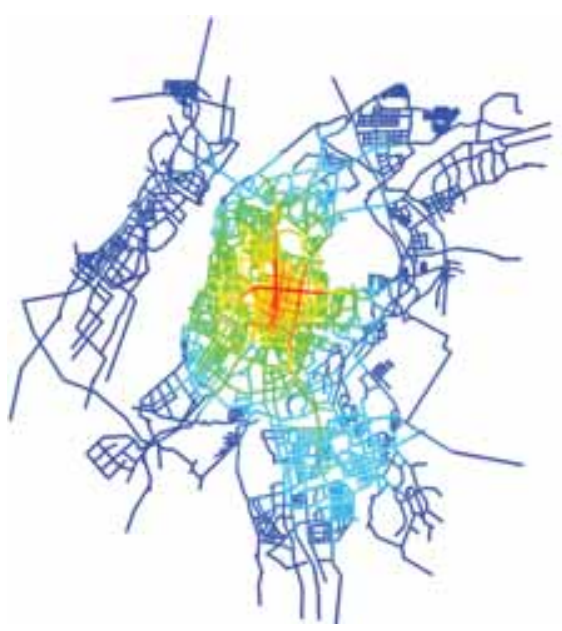

Figure A6. 53Integration at $8 \mathrm{~km}$ in 2009

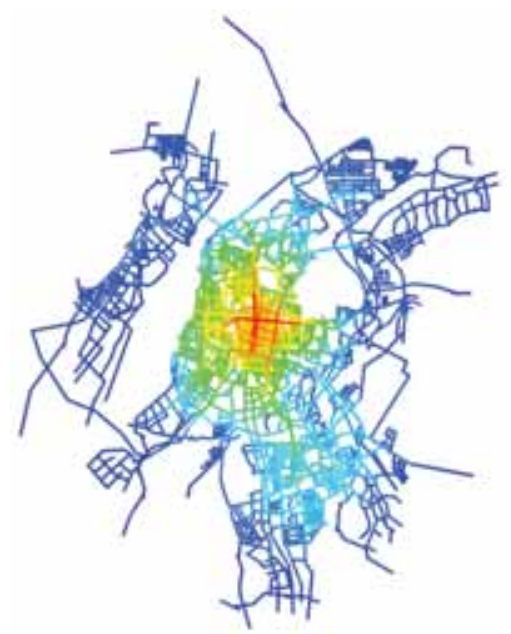

Figure A6. 54Integration at $8 \mathrm{~km}$ in 2010 


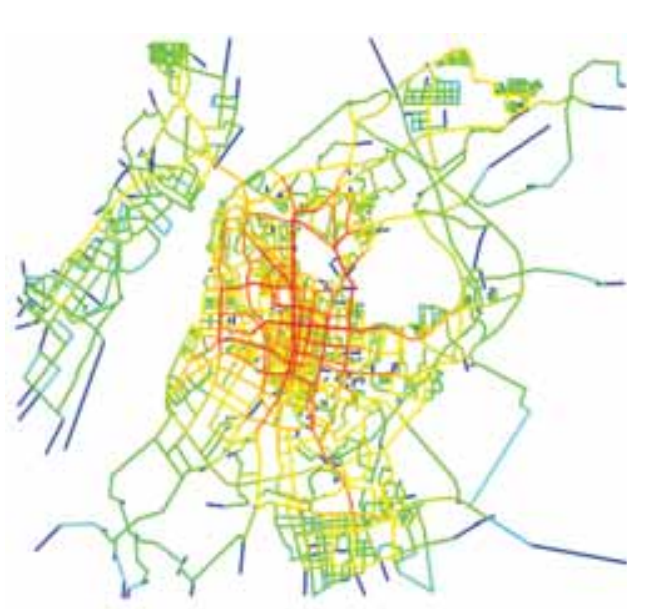

Figure A6. 55Choice at $8 \mathrm{~km}$ in 2005

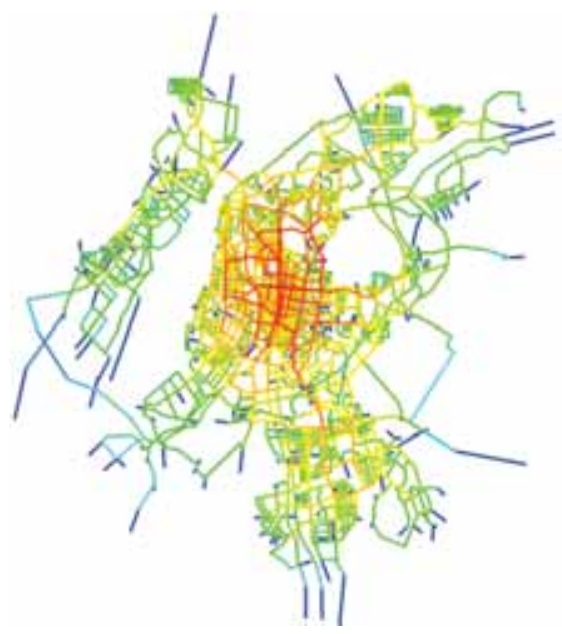

Figure A6. 56Choice at $8 \mathrm{~km}$ in 2006

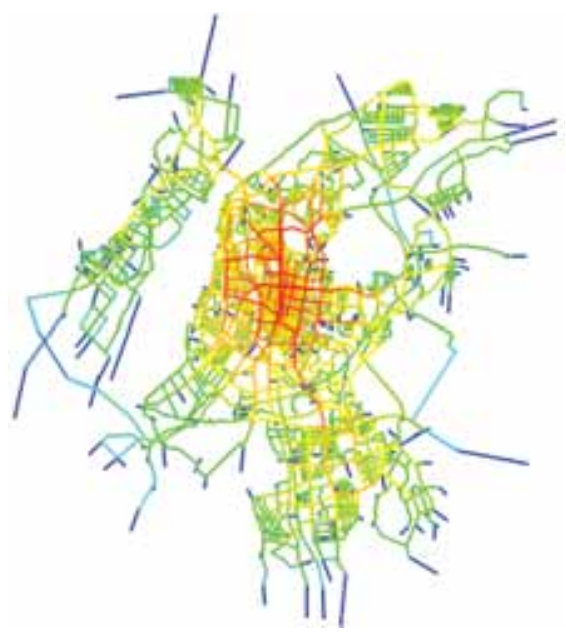

Figure A6. 57Choice at $8 \mathrm{~km}$ in 2007

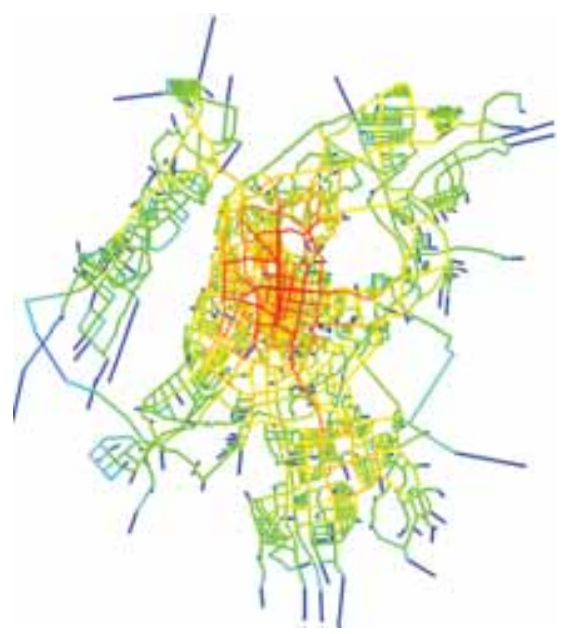

Figure A6. 58Choice at $8 \mathrm{~km}$ in 2008

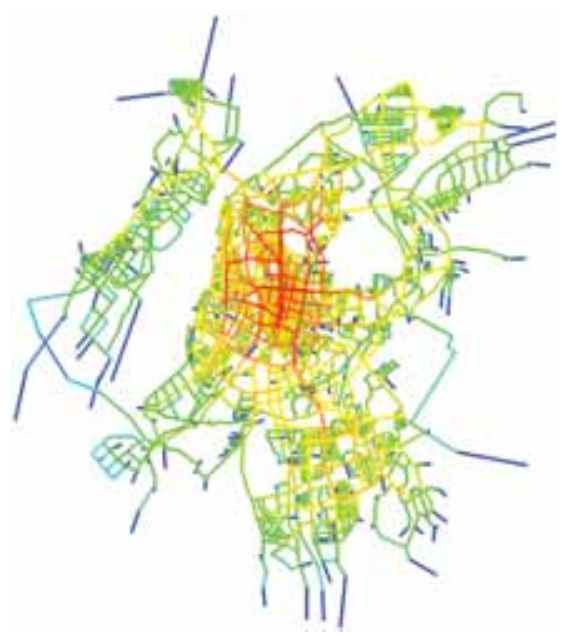

Figure A6. 59Choice at $8 \mathrm{~km}$ in 2009

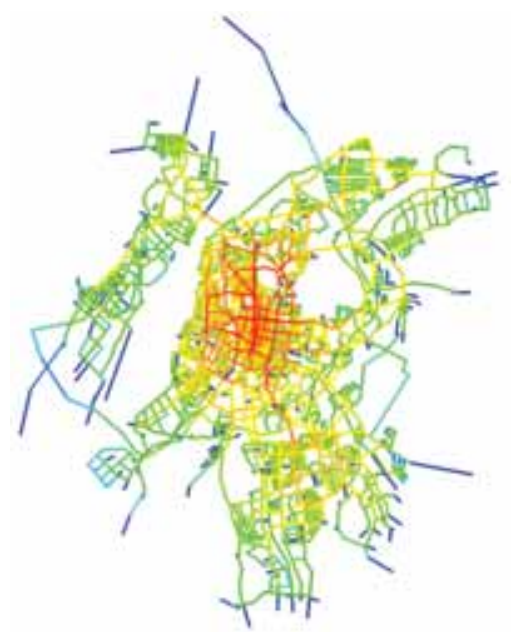

Figure A6. 60Choice at $8 \mathrm{~km}$ in 2010 


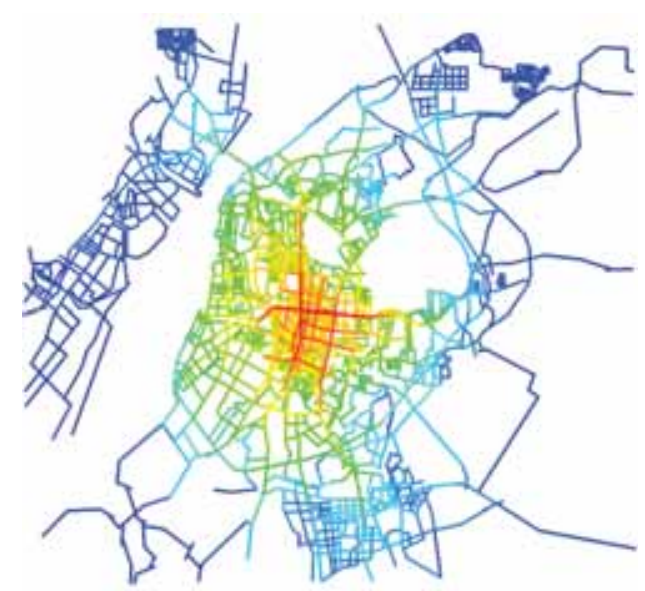

Figure A6. 61 Integration at $10 \mathrm{~km}$ in 2005

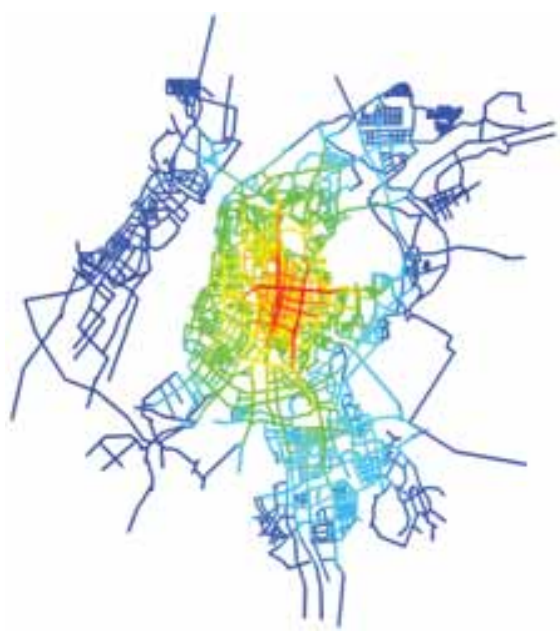

Figure A6. 62Integration at $10 \mathrm{~km}$ in 2006

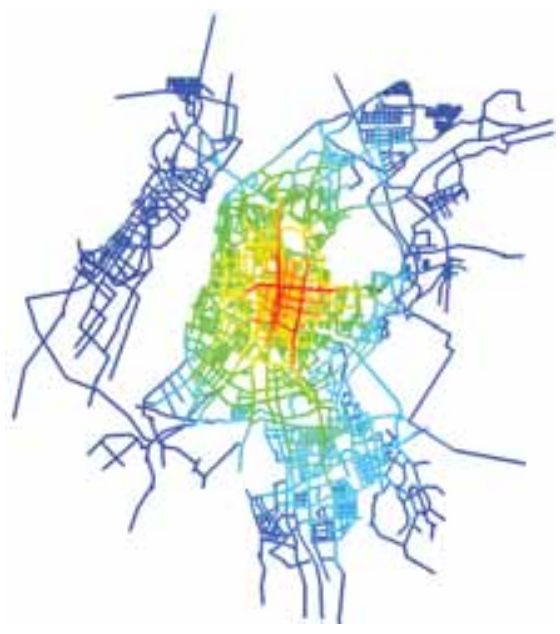

Figure A6. 63Integration at $10 \mathrm{~km}$ in 2007

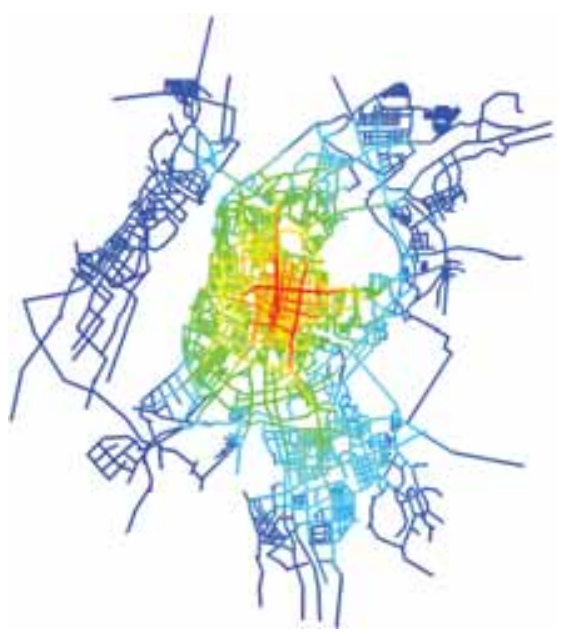

Figure A6. 64 Integration at $10 \mathrm{~km}$ in 2008

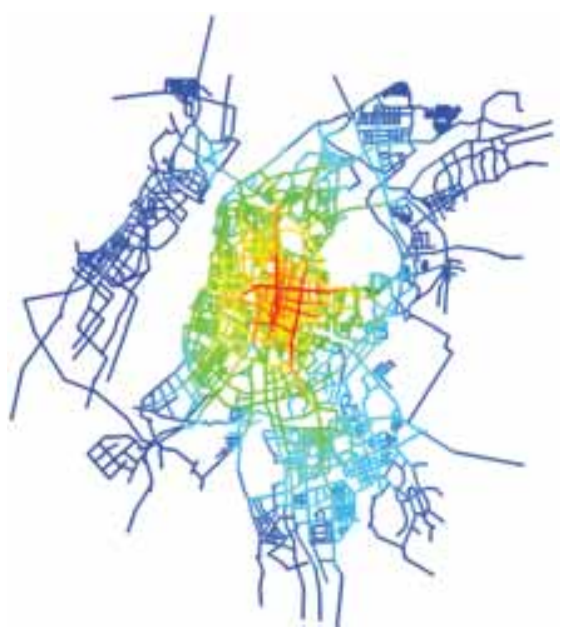

Figure A6. 65 Integration at $10 \mathrm{~km}$ in 2009

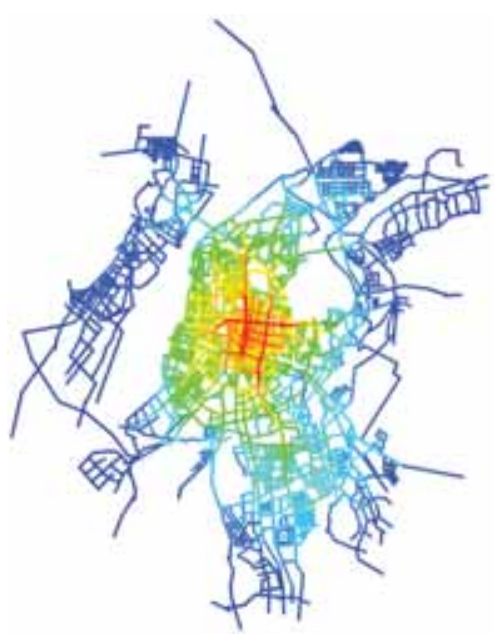

Figure A6. 66 Integration at $10 \mathrm{~km}$ in 2010 


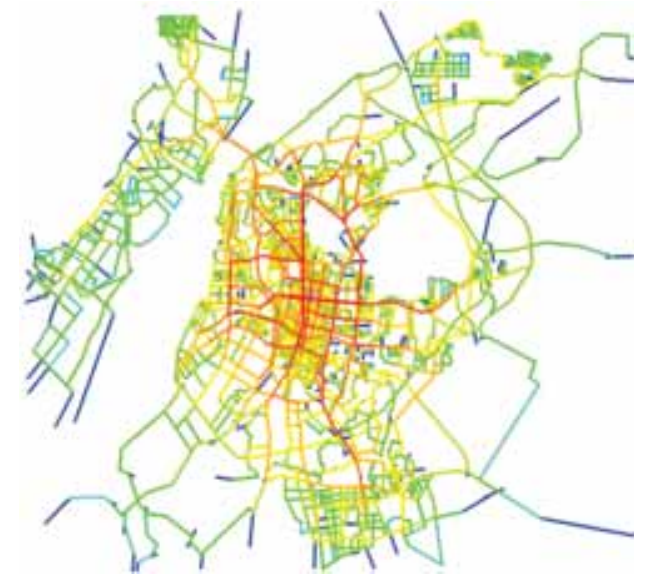

Figure A6. 67 Choice at $10 \mathrm{~km}$ in 2005

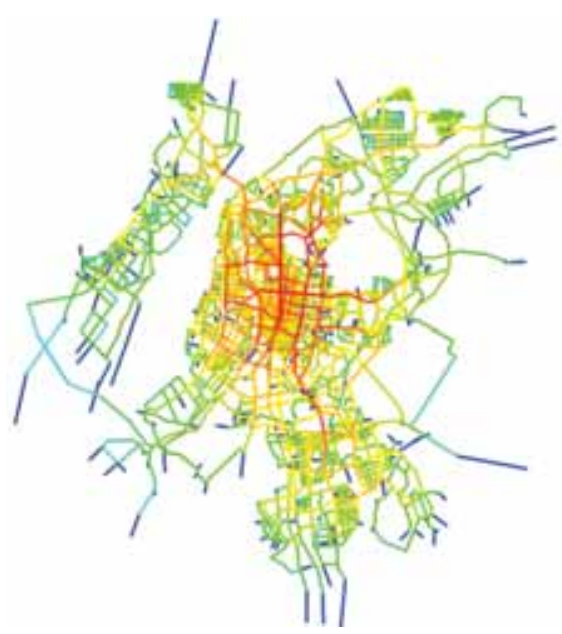

Figure A6. 68 Choice at $10 \mathrm{~km}$ in 2006

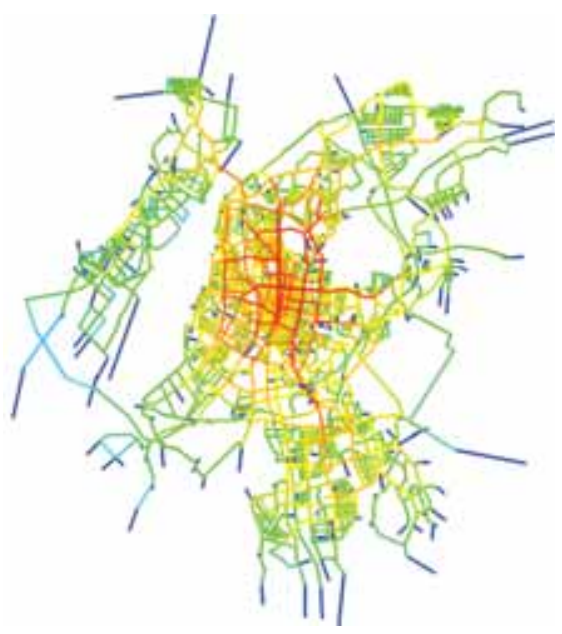

Figure A6. 69 Choice at $10 \mathrm{~km}$ in 2007

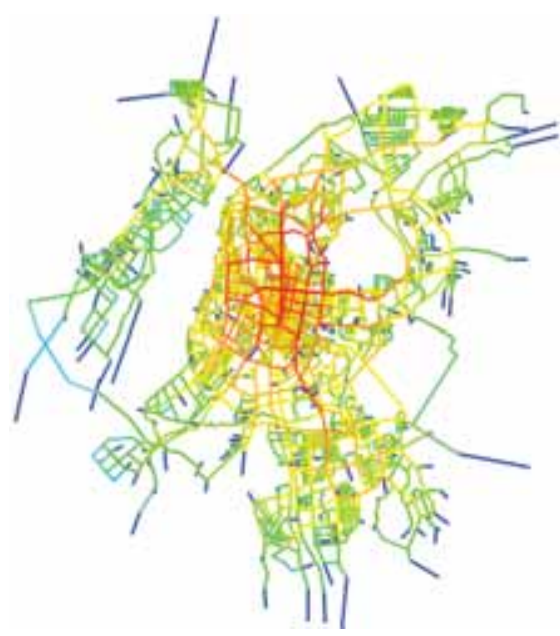

Figure A6. 70Choice at $10 \mathrm{~km}$ in 2008

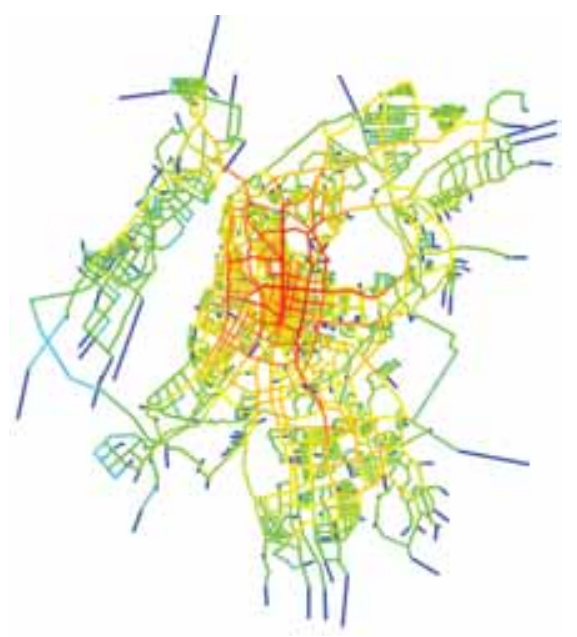

Figure A6. 71 Choice at $10 \mathrm{~km}$ in 2009

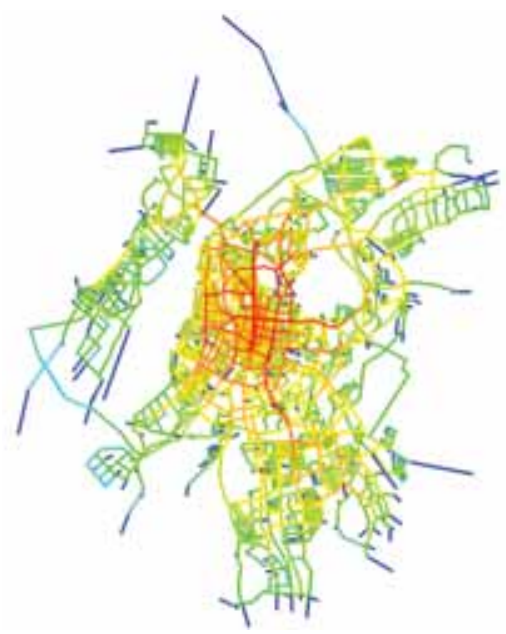

Figure A6. 72 Choice at $10 \mathrm{~km}$ in 2010 


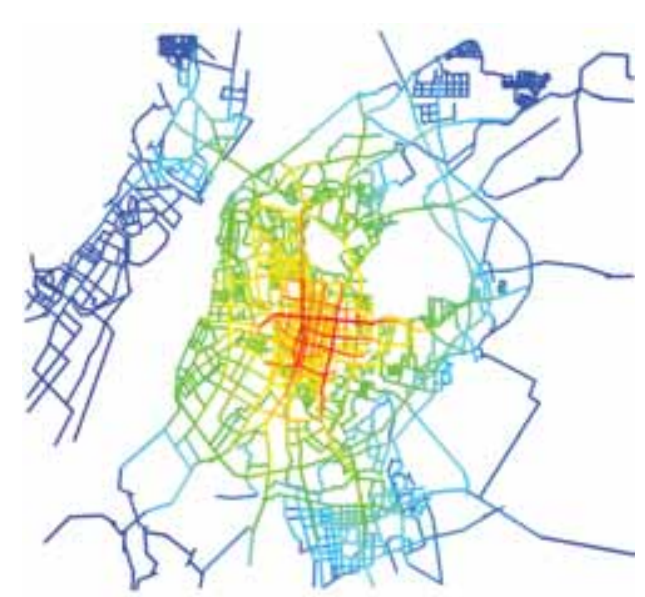

Figure A6. 73Integration at $12 \mathrm{~km}$ in 2005

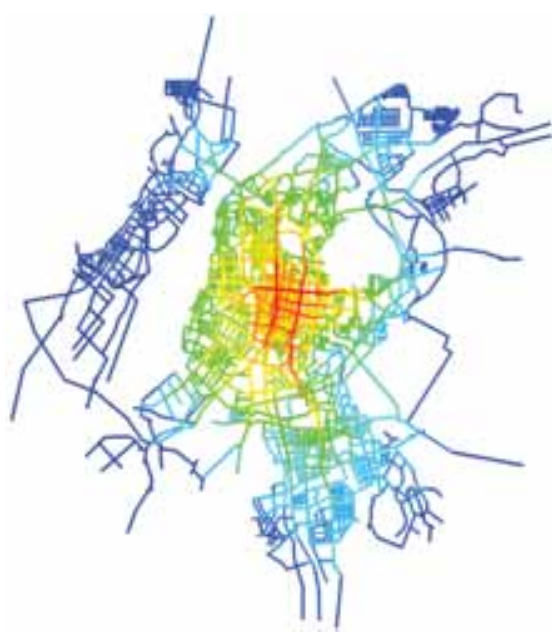

Figure A6. 74Integration at $12 \mathrm{~km}$ in 2006

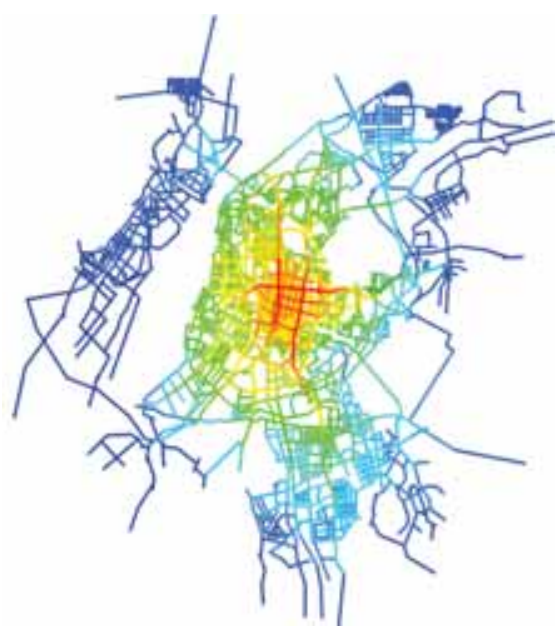

Figure A6. 75Integration at $12 \mathrm{~km}$ in 2007

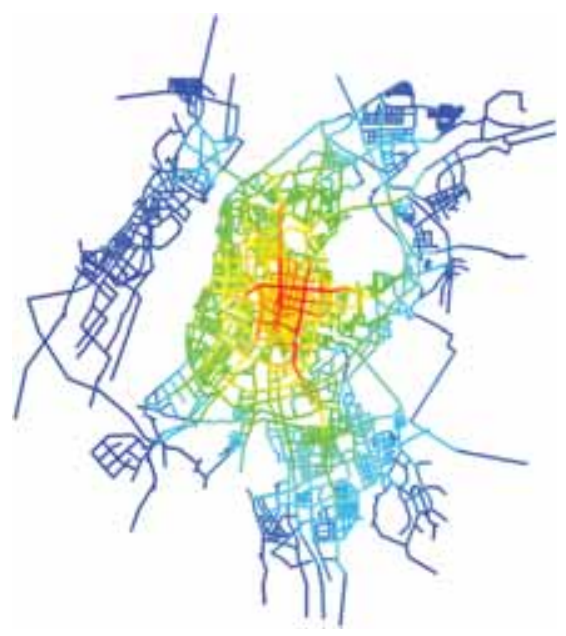

Figure A6. 76Integration at $12 \mathrm{~km}$ in 2008

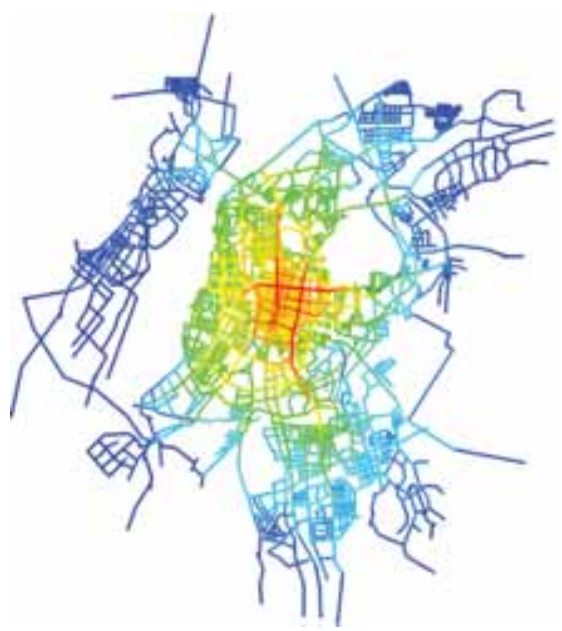

Figure A6. 77Integration at $12 \mathrm{~km}$ in 2009

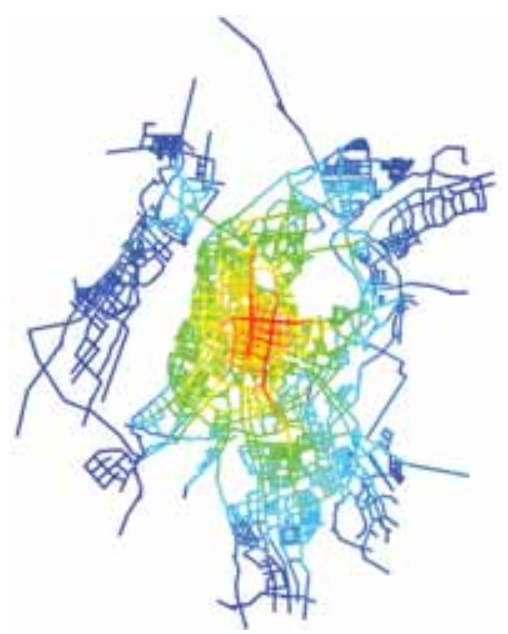

Figure A6. 78Integration at $12 \mathrm{~km}$ in 2010 


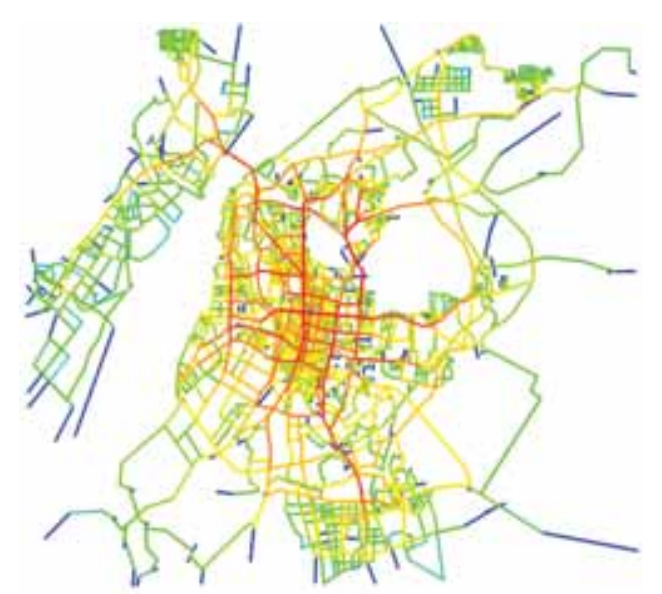

Figure A6. 79Choice at $12 \mathrm{~km}$ in 2005

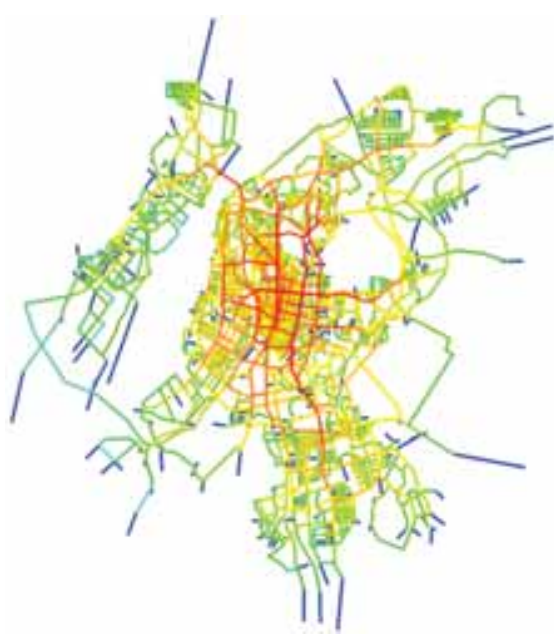

Figure A6. 80Choice at $12 \mathrm{~km}$ in 2006

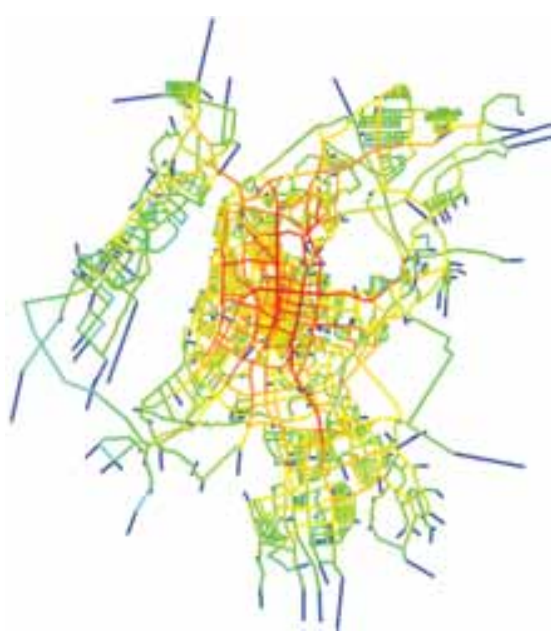

Figure A6. 81Choice at $12 \mathrm{~km}$ in 2007

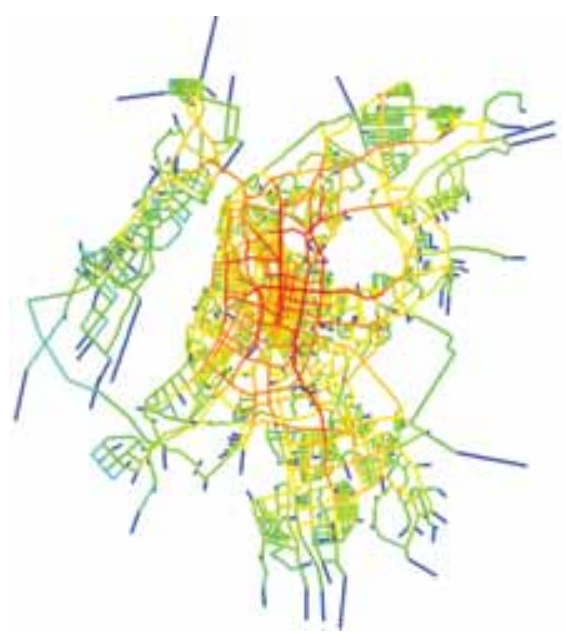

Figure A6. 82Choice at $12 \mathrm{~km}$ in 2008

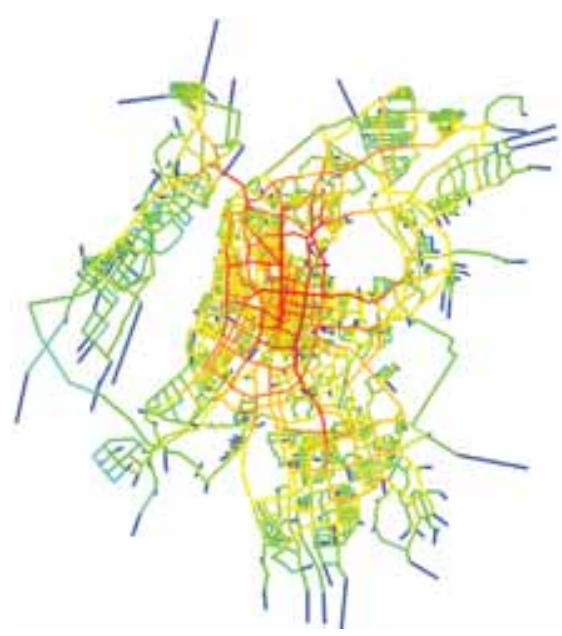

Figure A6. 83Choice at $12 \mathrm{~km}$ in 2009

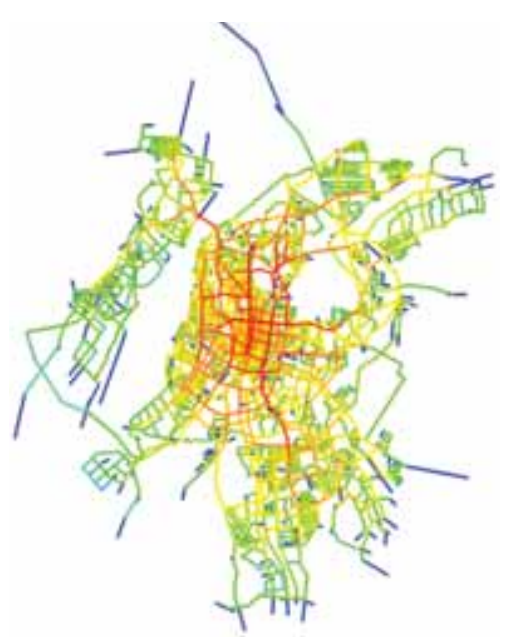

Figure A6. 84Choice at $12 \mathrm{~km}$ in 2010 


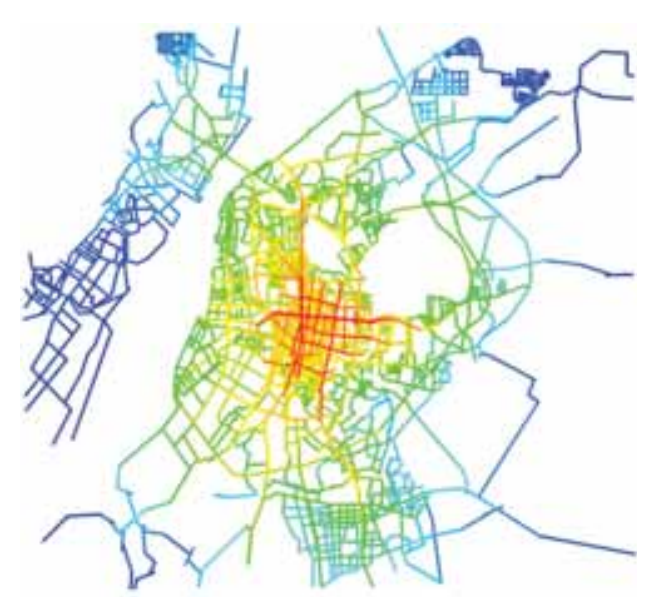

Figure A6. 85Integration at $15 \mathrm{~km}$ in 2005

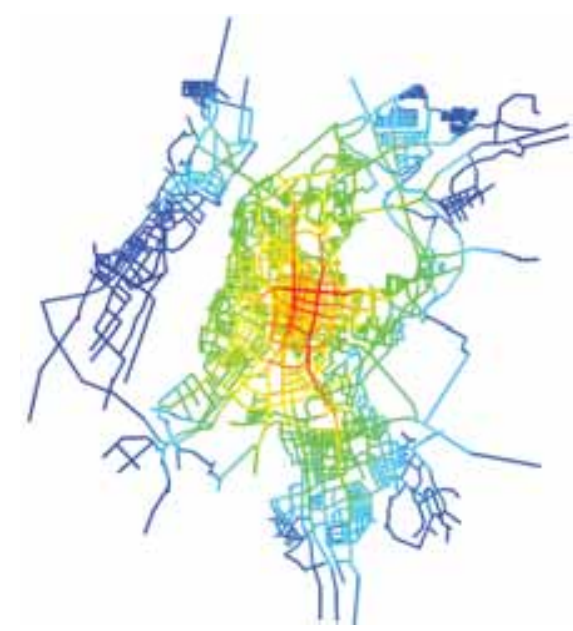

Figure A6. 86Integration at $15 \mathrm{~km}$ in 2006

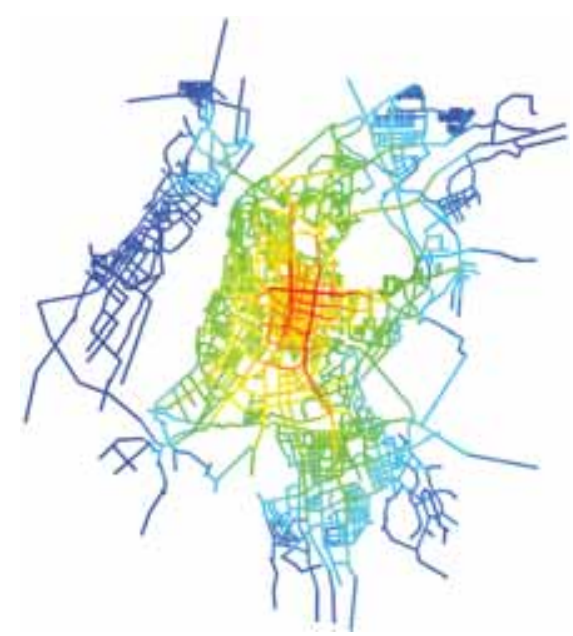

Figure A6. 87Integration at $15 \mathrm{~km}$ in 2007

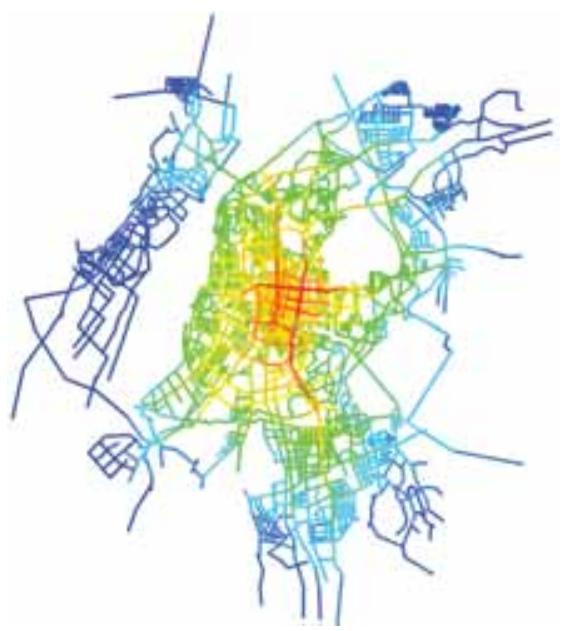

Figure A6. 88 Integration at 15 km in 2008

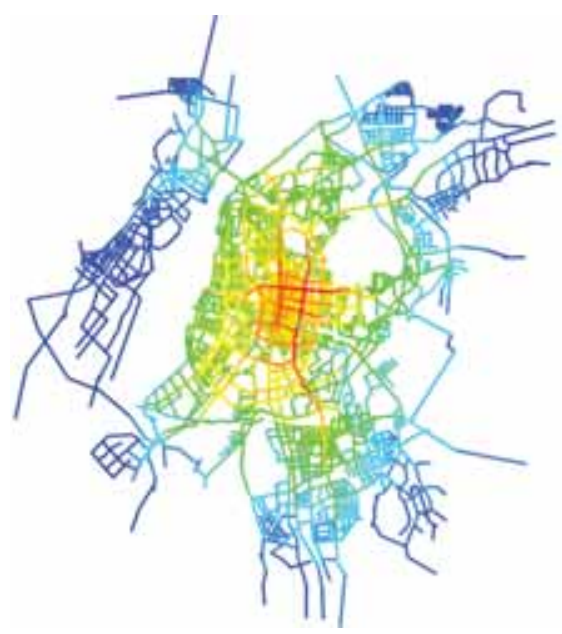

Figure A6. 89 Integration at 15 km in 2009

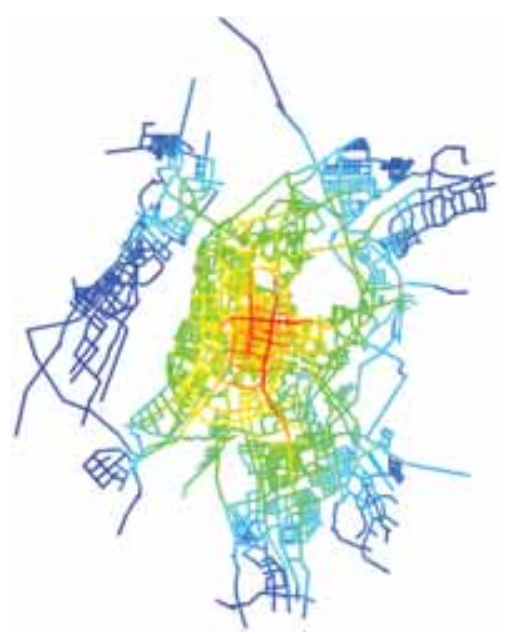

Figure A6. 90 Integration at 15 km in 2010 


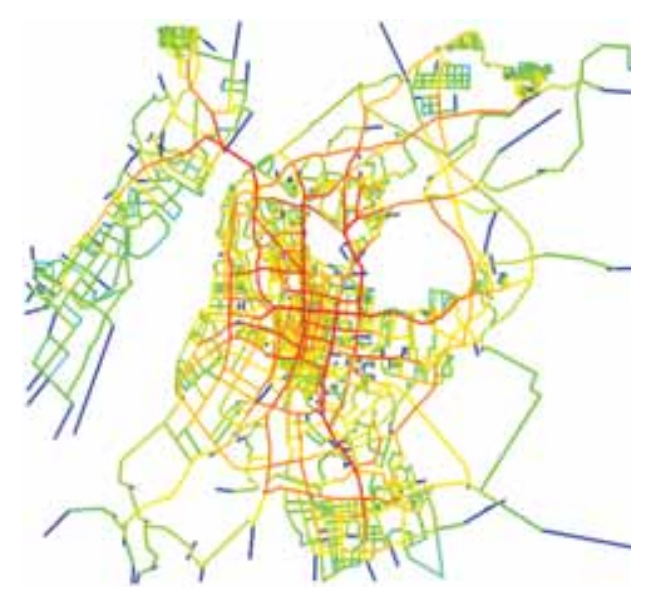

Figure A6. 91Choice at $15 \mathrm{~km}$ in 2005

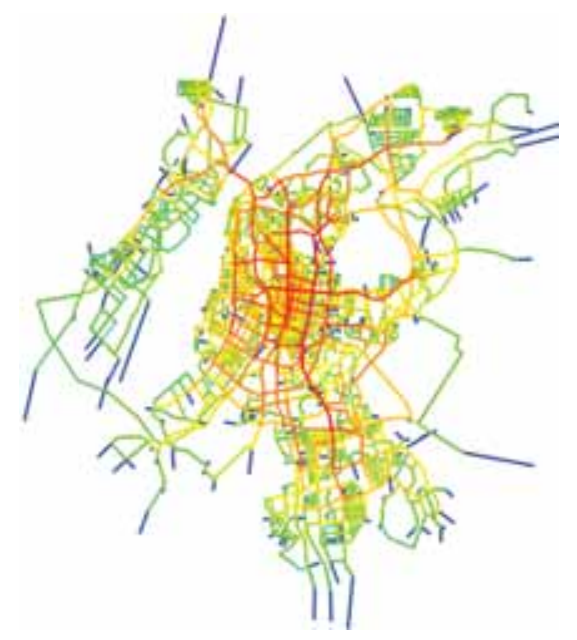

Figure A6. 92 Choice at $15 \mathrm{~km}$ in 2006

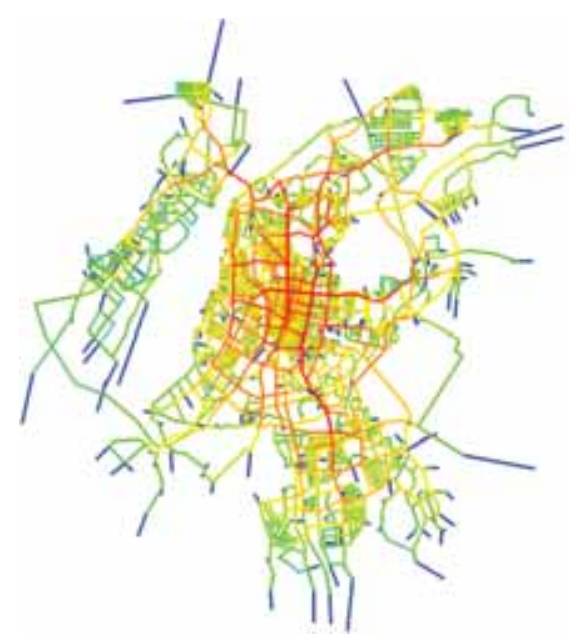

Figure A6. 93 Choice at $15 \mathrm{~km}$ in 2007

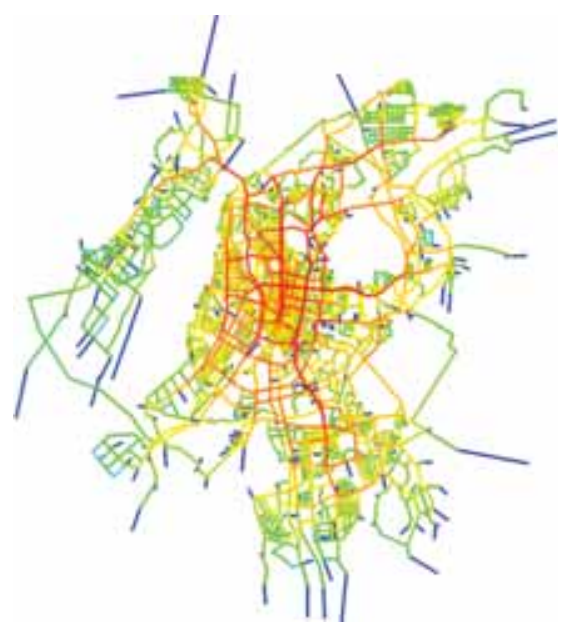

Figure A6. 94 Choice at $15 \mathrm{~km}$ in 2008

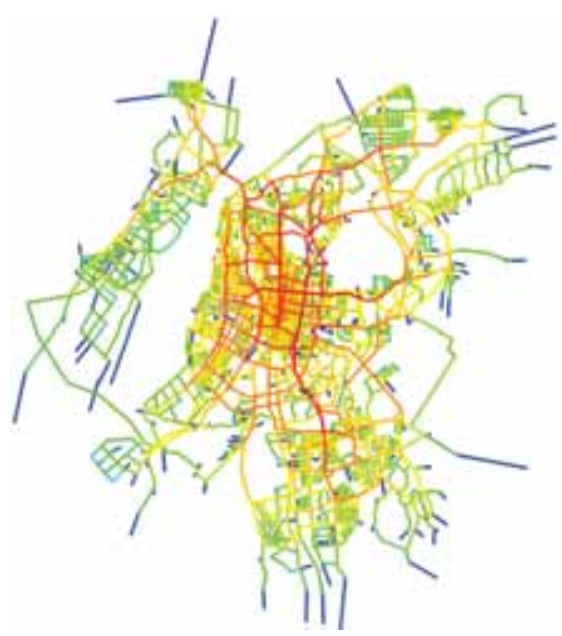

Figure A6. 95 Choice at $15 \mathrm{~km}$ in 2009

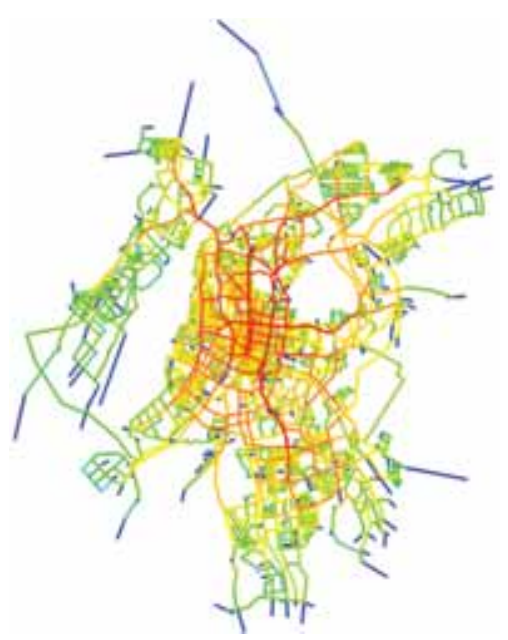

Figure A6. 96 Choice at $15 \mathrm{~km}$ in 2010 


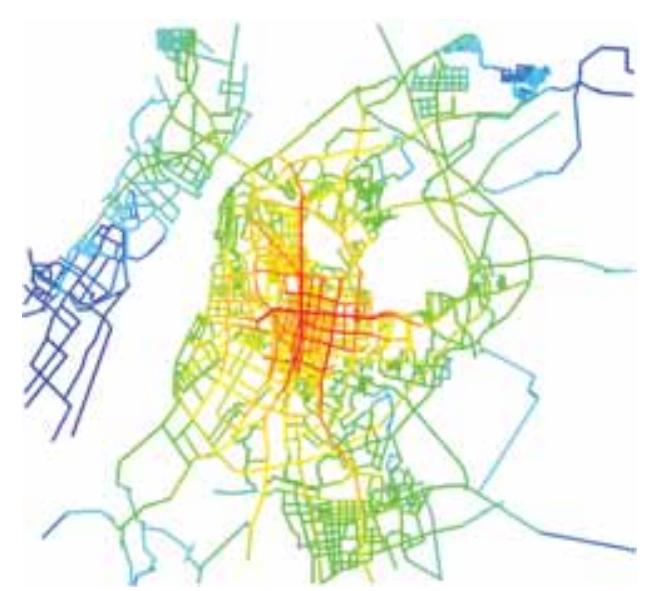

Figure A6. 97Integration at $20 \mathrm{~km}$ in 2005

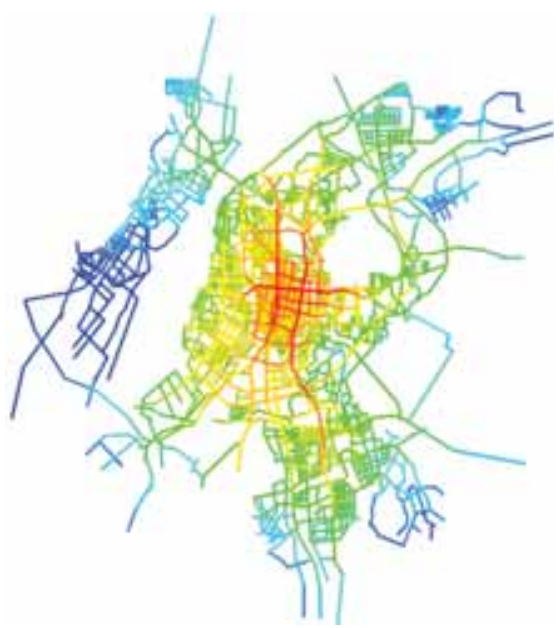

Figure A6. 98Integration at $20 \mathrm{~km}$ in 2006

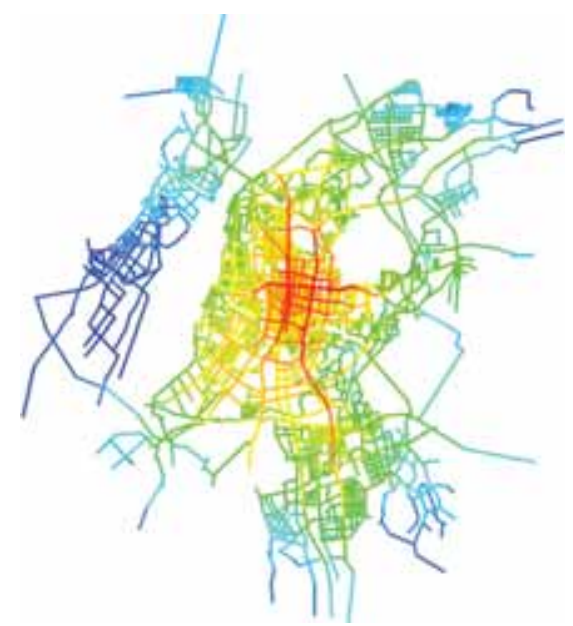

Figure A6. 99Integration at $20 \mathrm{~km}$ in 2007

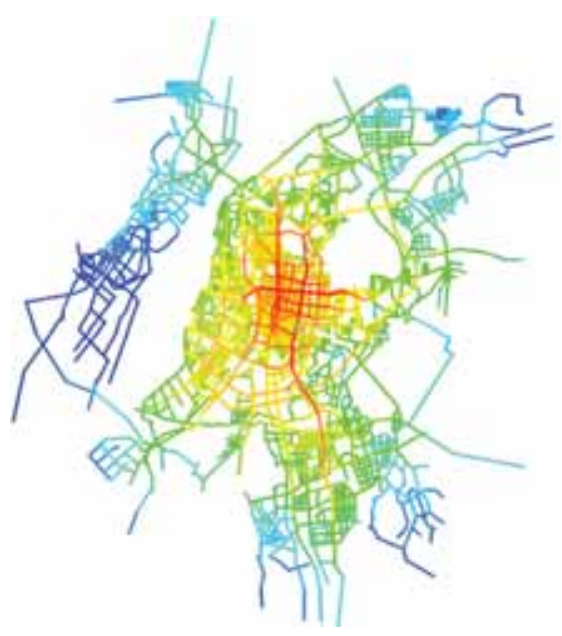

Figure A6. 100Integration at $20 \mathrm{~km}$ in 2008

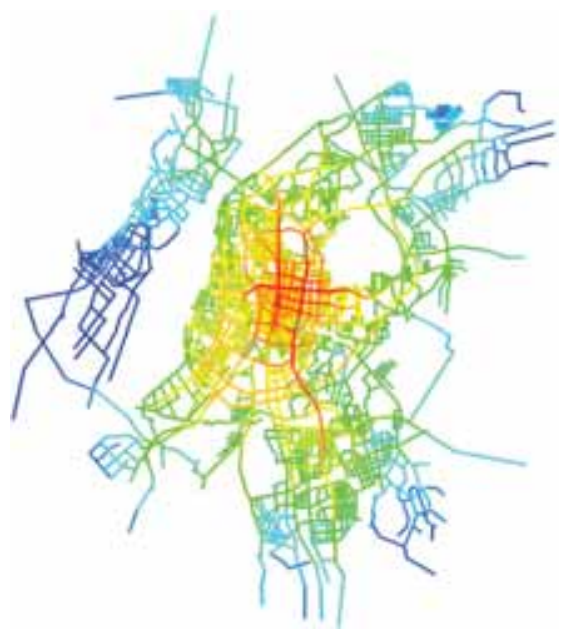

Figure A6. 101Integration at $20 \mathrm{~km}$ in 2009

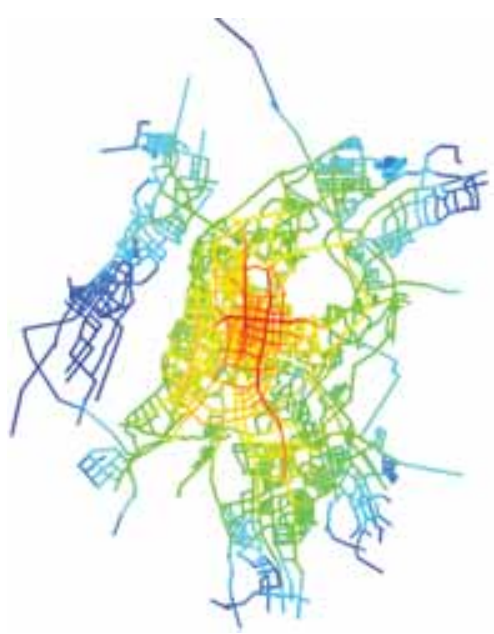

Figure A6. 102Integration at $20 \mathrm{~km}$ in 2010 


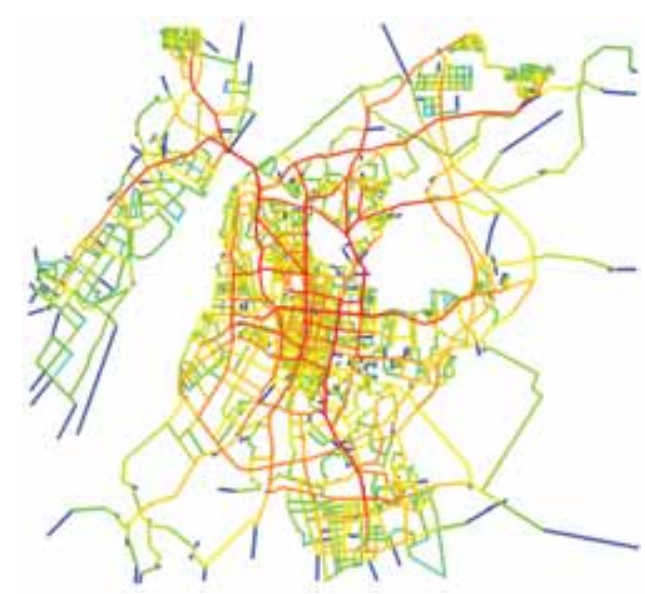

Figure A6. 103Choice at $20 \mathrm{~km}$ in 2005

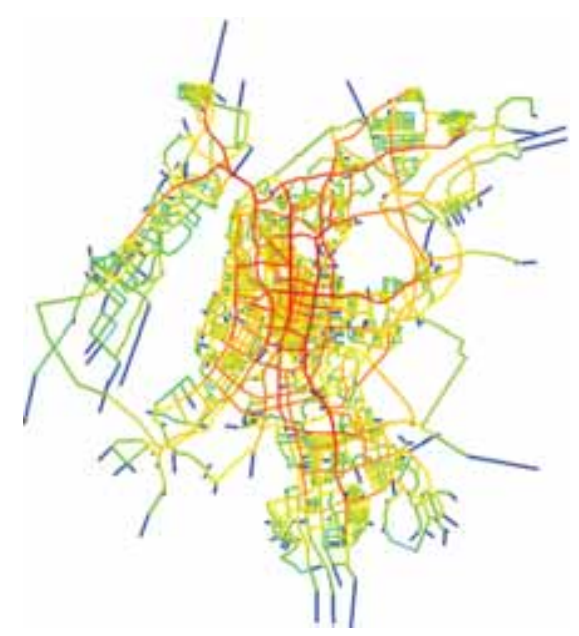

Figure A6. 104Choice at $20 \mathrm{~km}$ in 2006

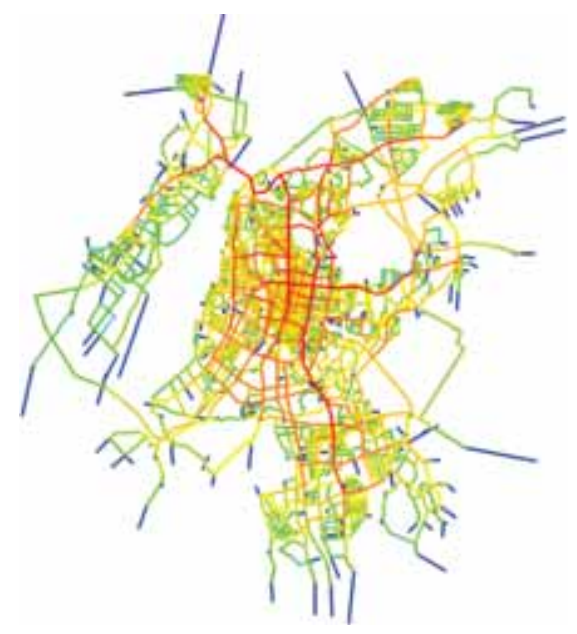

Figure A6. 105Choice at $20 \mathrm{~km}$ in 2007

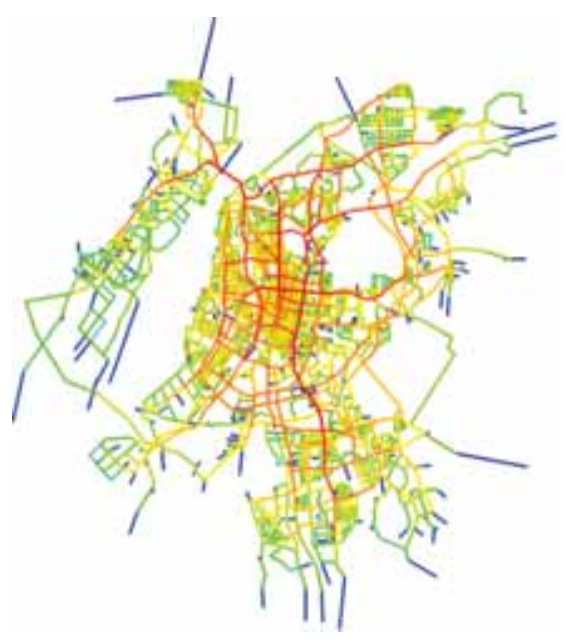

Figure A6. 106Choice at $20 \mathrm{~km}$ in 2008

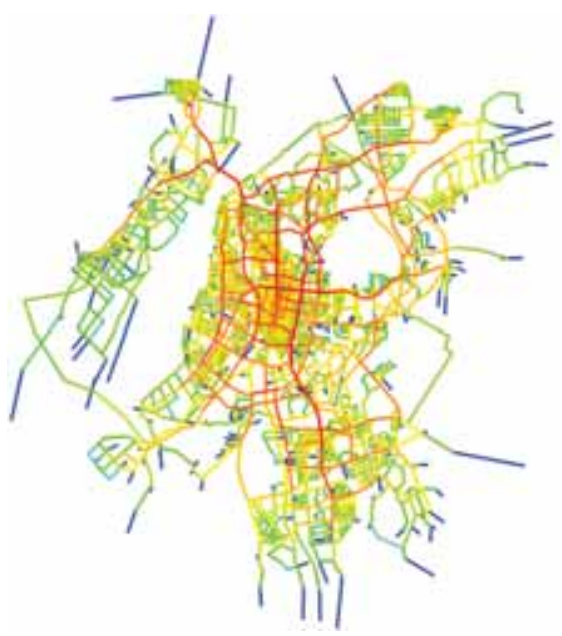

Figure A6. 107Choice at $20 \mathrm{~km}$ in 2009

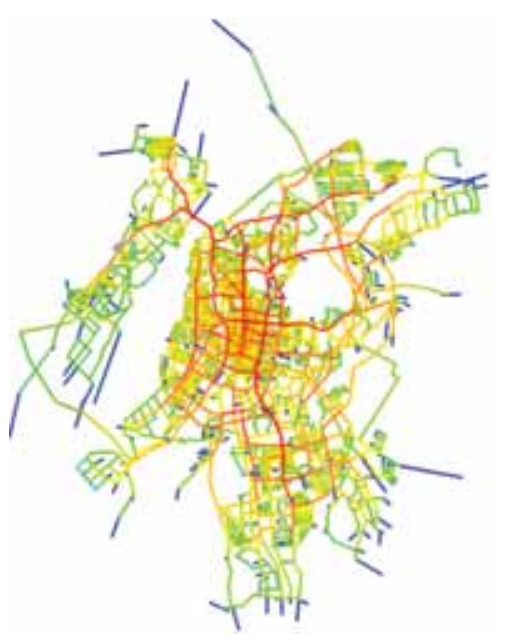

Figure A6. 108Choice at 20km in 2010 


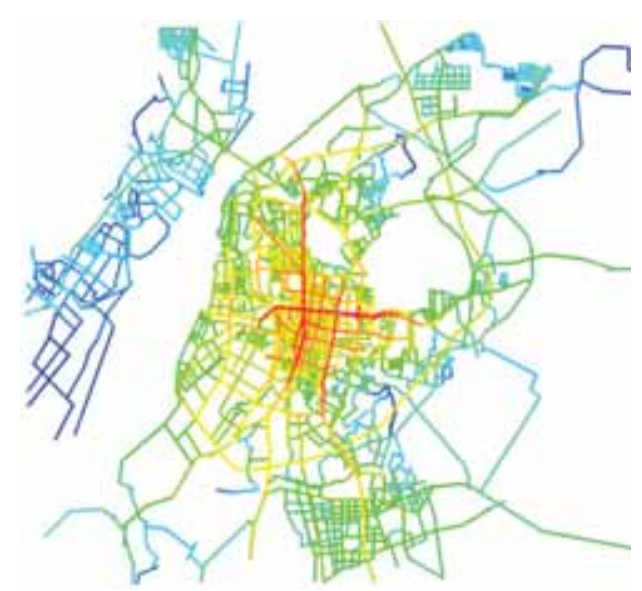

Figure A6. 109 Global integration in 2005

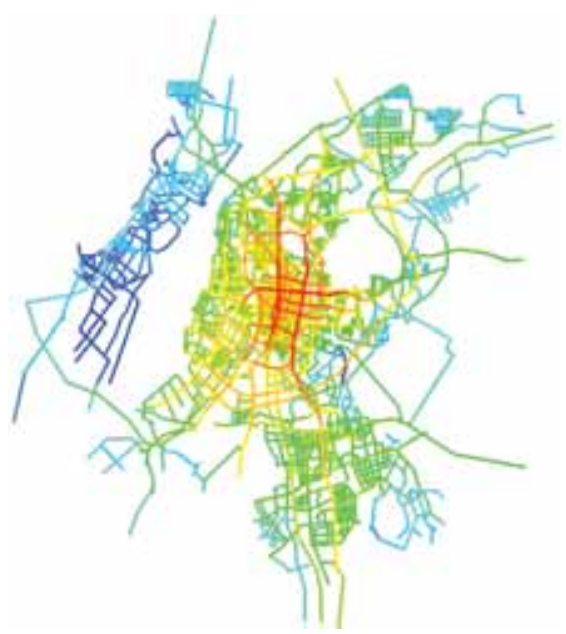

Figure A6. 110Global integration in 2006

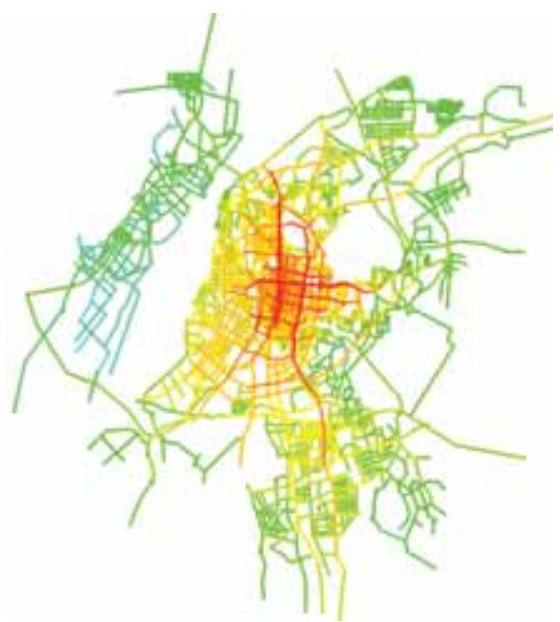

Figure A6. 111Global integration in 2007

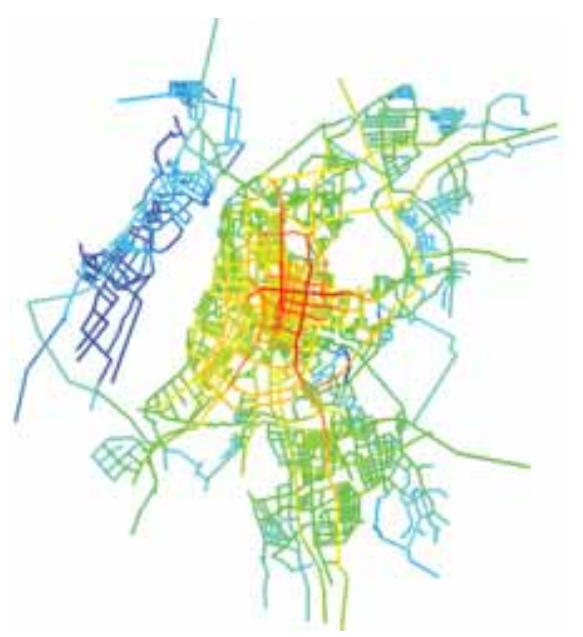

Figure A6. 112Global integration in 2008

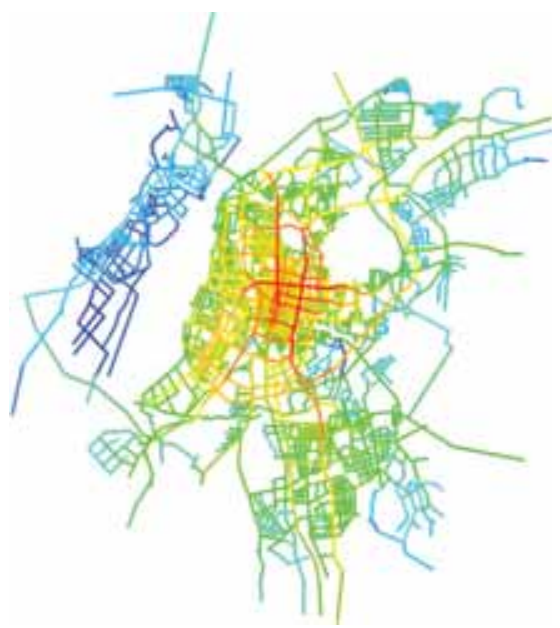

Figure A6. 113Global integration in 2009

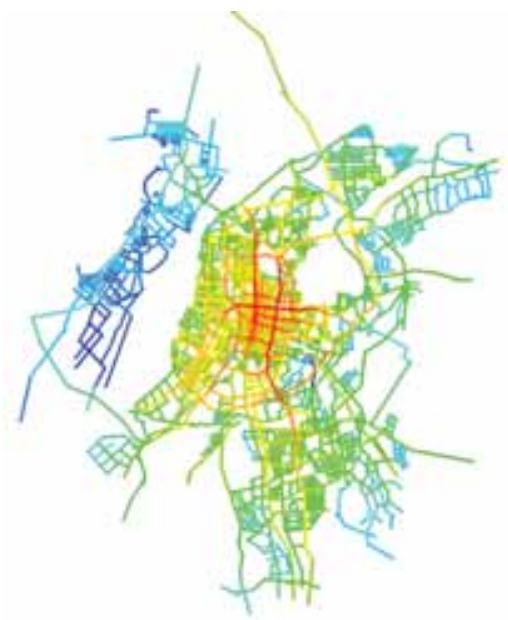

Figure A6. 114Global integration in 2010 


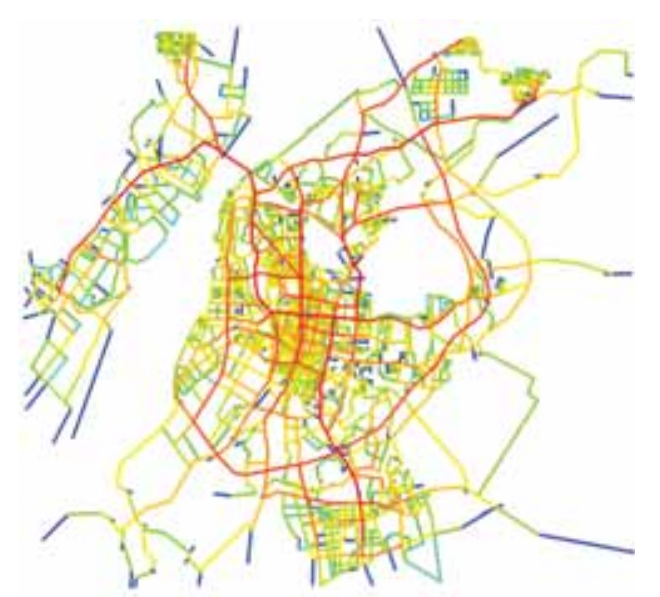

Figure A6. 115Global choice in 2005

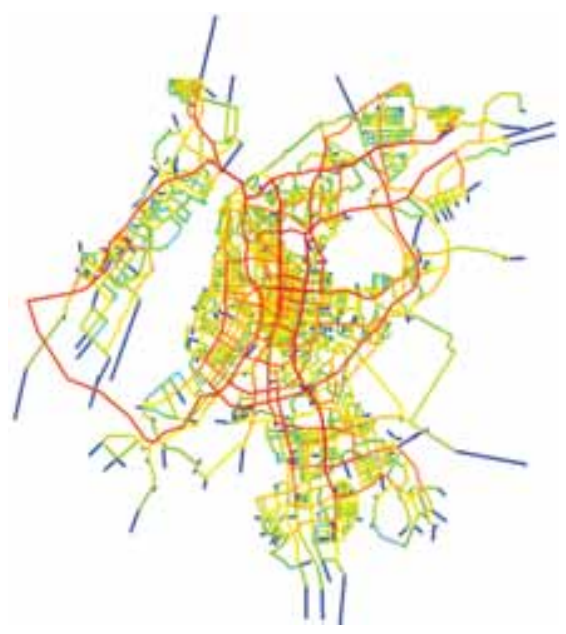

Figure A6. 116Global choice in 2006

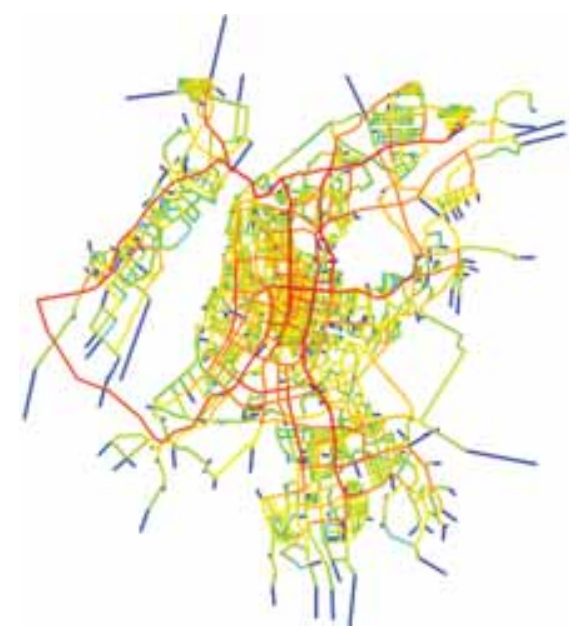

Figure A6. 117Global choice in 2007

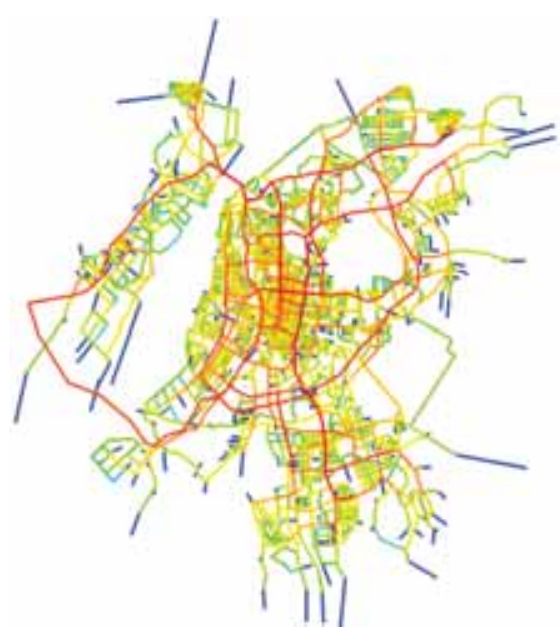

Figure A6. 118Global choice in 2008

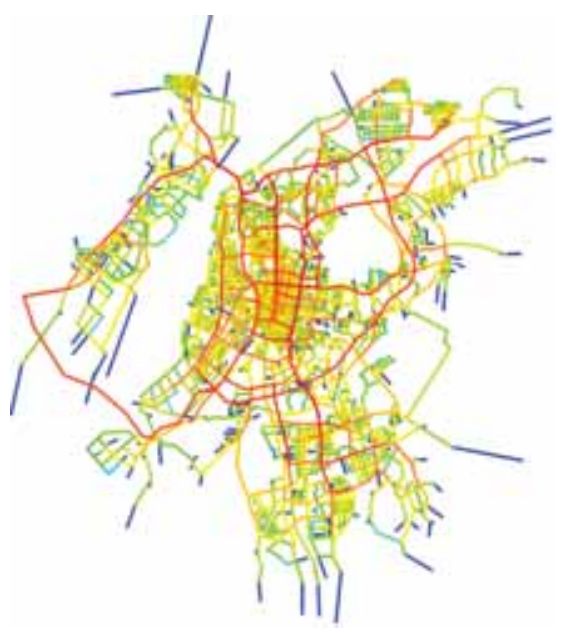

Figure A6. 119Global choice in 2009

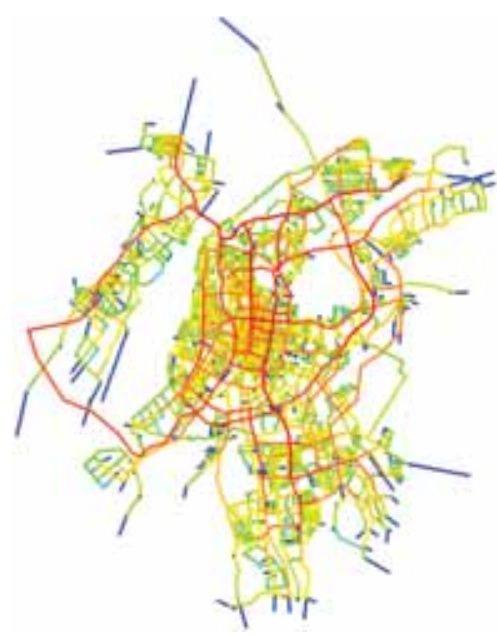

Figure A6. 120Global choice in 2010 